Published in Rep. Prog. Phys. 61, 889-997 (1998).

\title{
Analogue studies of nonlinear systems
}

\author{
D G Luchinsky†§, P V E McClintock † and M I Dykman $\ddagger$ \\ $\dagger$ Department of Physics, Lancaster University, Lancaster LA1 4YB, UK \\ $\ddagger$ Department of Physics and Astronomy, Michigan State University, East Lansing, MI 48824, USA
}

Received 23 February 1998

\begin{abstract}
The design of analogue electronic experiments to investigate phenomena in nonlinear dynamics, especially stochastic phenomena, is described in practical terms. The advantages and disadvantages of this approach, in comparison to more conventional digital methods, are discussed. It is pointed out that analogue simulation provides a simple, inexpensive, technique that is easily applied in any laboratory to facilitate the design and implementation of complicated and expensive experimental projects; and that there are some important problems for which analogue methods have so far provided the only experimental approach. Applications to several topical problems are reviewed. Large rare fluctuations are studied through measurements of the prehistory probability distribution, thereby testing for the first time some fundamental tenets of fluctuation theory. It has thus been shown for example that, whereas the fluctuations of equilibrium systems obey time-reversal symmetry, those under non-equilibrium conditions are temporally asymmetric. Stochastic resonance, in which the signal-to-noise ratio for a weak periodic signal in a nonlinear system can be enhanced by added noise, has been widely studied by analogue methods, and the main results are reviewed; the closely related phenomena of noise-enhanced heterodyning and noiseinduced linearization are also described. Selected examples of the use of analogue methods for the study of transient phenomena in time-evolving systems are reviewed. Analogue experiments with quasimonochromatic noise, whose power spectral density is peaked at some characteristic frequency, have led to the discovery of a range of interesting and often counter-intuitive effects. These are reviewed and related to large fluctuation phenomena. Analogue studies of two examples of deterministic nonlinear effects, modulation-induced negative differential resistance (MINDR) and zero-dispersion nonlinear resonance (ZDNR) are described. Finally, some speculative remarks about possible future directions and applications of analogue experiments are discussed.
\end{abstract}




\section{Contents}

1. Introduction 891

2. Technical details $\quad 893$

2.1. Circuit design 893

2.2. Noise generators $\quad 898$

2.3. Signal acquisition and analysis $\quad 900$

3. Large rare fluctuations 901

3.1. The concept of large fluctuations 901

3.2. Prehistory probability distribution 904

3.3. Time asymmetry of classical fluctuations $\quad 915$

3.4. Status of the experiments on large fluctuations 928

4. Stochastic resonance 929

4.1. What is stochastic resonance? 929

4.2. Stochastic resonance as a linear response phenomenon 931

4.3. Non-conventional forms of stochastic resonance 936

4.4. Noise-enhanced heterodyning 954

4.5. Noise-induced linearization 958

5. Transient effects 961

5.1. Swept-parameter systems 961

5.2. Decay of unstable states and transient multimodality 965

6. Phenomena induced by quasimonochromatic noise 972

6.1. Quasimonochromatic noise and its generation 972

6.2. Transitions in a bistable potential 974

6.3. The stationary distribution 976

6.4. Large fluctuations and observation of a switching point 979

7. Deterministic nonlinear phenomena 981

7.1. Modulation-induced negative differential resistance 982

7.2. Zero-dispersion nonlinear resonance 984

8. Future directions 989

Acknowledgments 990

$\begin{array}{ll}\text { References } & 990\end{array}$ 


\section{Introduction}

Analogue simulation provides a method of solving complicated dynamical equations. One builds an electronic model of the system under study and then investigates its behaviour, usually under the influence of external forces of some kind, using a digital data processor to analyse the response. This approach has been found especially useful in relation to stochastic problems, i.e. those where the system of interest is subject to random fluctuations (noise), either of external or internal origin. Contrary to a still widespread misapprehension, analogue simulation does not require large or costly apparatus: an 'analogue computer' as such is no longer needed. Nor is the technique difficult to implement. In this review, we explain the basis of the method and describe how different variants have been used to address some selected problems in (mostly) stochastic nonlinear dynamics. First, however, we discuss in more detail why analogue modelling techniques are valuable, and their advantages and disadvantages as compared to more commonly used digital methods (Mannella 1997).

A major advantage of analogue simulation is that it readily enables large volumes of a system's parameter space to be surveyed quickly for interesting phenomena, often by turning knobs to adjust the relevant parameters while examining the results on a visual display; the equivalent procedure with a digital system is often slower and more ponderous. Usually analogue simulations are used to test theoretical predictions. However, there are many examples of new physical phenomena and ideas first discovered through analogue simulations. When noise is added to a strongly nonlinear system, for example, it may improve not only the transmission of signals through the system, but also the signal-tonoise ratio measured at the output (Fauve and Heslot 1983) — an observation that in a large part was responsible for the outburst of work on stochastic resonance (see section 4 later). Other examples include the idea for the Josephson voltage standard (Kautz 1988, 1996), and the observations of skewing in the stochastic phase portraits of bistable systems driven by coloured noise (Moss and McClintock 1985, Moss et al 1986), modulation-induced negative differential resistance (Dow et al 1987) and noise-induced spectral narrowing (Dykman et al 1990a, b). In each of these cases, the phenomenon had not been predicted theoretically, was not anticipated prior to the experiments and appeared unexpectedly in the analogue model.

In a sense, analogue simulations lie in between real experiments and digital simulations, and combine certain characteristic features of each. In both forms of simulation one investigates model systems. However, the techniques for data acquisition and processing used in analogue simulation are often exactly the same as those used in the corresponding experiments on real physical systems. This feature can sometimes be extremely useful, for example in that it enables the acquisition/analysis software to be developed, tested and implemented in studies of an analogue model prior to implementing it in the more complicated environment of a real experiment. Noise-enhanced heterodyning in a fluctuating nonlinear optical element, for example, was first simulated in an idealized electronic model (Dykman et al 1994a) prior to performing the nonlinear optical experiment itself (Dykman et al 1995a, b).

There is a long-standing and close relationship between studies of fluctuating nonlinear systems and experiments on electrical circuits. The connections were studied in radioengineering over several decades, a typical example being the work on fluctuations in the Thompson generator (Rytov 1956a,b). In fact, it was experiments of this kind on electronic circuits that were largely responsible for stimulating the development of the theory of random processes as a whole, yielding a variety of important results some of which are now being used in quantum electronics. The relationships between electrical circuits 
and the properties of Langevin and Fokker-Planck equations were discussed by Landauer (1962) and Stratonovich $(1963,1967)$. In analogue experiments, the static properties of the electronic system are known (or are assumed to be known), and the problem is to investigate how it responds to random or periodic forcings. An example of the revealing role of such simulations can be seen from the analysis (Smythe et al 1983a, McClintock and Moss 1985, Moss et al 1985b) of the Ito-Stratonovich dilemma (Van Kampen 1990). It concerns the description of how a system responds to white noise. Any naturally occurring noise necessarily has a finite correlation time. This is true for the noise used in analogue simulations. Noise in Langevin equations can be considered effectively white in the limit where its correlation time is very small compared to the relaxation times of the system, which corresponds to the Stratonovich prescription. The physical relevance of this prescription was clearly demonstrated in the analogue simulations. The authors concluded that the alternative Ito prescription, much used by theorists at that time, was inapplicable to real physical systems except perhaps in special cases (e.g. equations with delay). In contrast, when trying to implement similar studies on a digital computer, with the values of the noise being uncorrelated at different discretized instants of time, it was quickly realized that any attempt to resolve the dilemma would be fruitless-because it would be necessary for the programmer to choose either the Ito or Stratonovich stochastic calculus in writing the simulation code, thus pre-selecting the answer.

In contrast to digital simulations, truncation errors do not accumulate in analogue simulations. Analogue simulations are therefore especially valuable for use, for example, with fast oscillating systems where the integration time (the time over which data are accumulated and perhaps ensemble-averaged) substantially exceeds the vibration period, as in problems involving quasimonochromatic noise (Dykman et al 1991b, 1993e, and section 6 later). Although digital techniques can always in principle be made more accurate than analogue methods, which typically achieve 2-3\% accuracy, the relative simplicity of analogue simulations and their high speed represent significant advantages, particularly where qualitative results have to be established - as was the case for the ring-laser gyroscope equation (Vogel et al 1987a), for example, where it was found that analogue results of satisfactory statistical quality could be acquired in a matter of 15-30 min, whereas numerical solution of the Fokker-Planck equation took hours of Cray time.

Finally, experience suggests that digital and analogue methods should be regarded as complementary techniques for the study of stochastic nonlinear problems. Each has its own advantages and disadvantages; which of these is emphasized or de-emphasized will depend on the nature of the system being investigated. As in any experimental study, it is possible to make mistakes and generate artifacts using either form of simulation. For really complicated problems it is therefore desirable to use both techniques if possible, with one acting as a check on the other, to eliminate mistakes or hidden flaws in the algorithm used for the digital simulation, or misconnections and experimental uncertainties in the analogue circuit.

In what follows, although we will concentrate mostly on analogue experimental investigations by the Lancaster group, we would emphasize that the technique has been, and continues to be, used successfully in many other laboratories. For example, electronic models have been employed extensively over many years for studies of deterministic phenomena such as heart rate variability (Van der Pol and Van der Mark 1928), chaos (Holmes 1979, Linsay 1981, Testa et al 1982, Jeffries and P'erez 1982, Yeh and Kao 1982, D'Humieres et al 1982, Robinson 1990, King and Gaito 1992, Gomes and King 1992, Heagy et al 1994), phenomena near period-doubling bifurcations (Jeffries and Wiesenfeld 1985, Bryant and Wiesenfeld 1986, Vohra and Bucholtz 1993, Vohra et al 1994), and the three- 
photon Josephson junction parametric amplifier (Bryant et al 1987). Modelling techniques for noise-driven systems were developed by a number of scientists and mathematicians including, particularly, Morton and Corrsin (1969). The first applications of the technique in its contemporary form were probably those reported by Sancho et al (1982), Arecchi et al (1982), Fauve and Heslot (1983) and Smythe et al (1983a, b). Subsequently, stochastic analogue modelling has been used by numerous research groups including, for example, Mitschke et al (1985), Fronzoni et al (1985), Gammaitoni et al (1989), Anishchenko et al (1993) and Carroll and Pecora (1993).

The particular topics chosen as examples for discussion are intended to be representative. We point out, however, that we have successfully applied the analogue approach to the study of many other interesting stochastic nonlinear problems, in addition to those mentioned, including: noise-induced phase transitions (Smythe et al 1983a), stochastic postponements of critical onsets (Robinson et al 1985); noise-induced changes in the relaxation times of bistable systems driven by both white (Sancho et al 1985) and coloured (Casademunt et al 1987) parametric (i.e. multiplicative) noise; bistability driven by coloured noise (Hanggi et al 1985, Fronzoni et al 1986); noise-induced postponements of bifurcations in a ring laser model (Mannella et al 1987a, c) and in the Brusselator (Fronzoni et al 1987a); the effect of noise on a Hopf bifurcation (Fronzoni et al 1987b); fluctuation spectra of the double-well Duffing oscillator (Mannella et al 1987b, Dykman et al 1988); Fokker-Planck descriptions of coloured noise-driven processes (Grigolini et al 1988); relaxation of fluctuations in the steady state of the Stratonovich model (Mannella et al 1988); the effect of noise on the Fréedericksz transition (Stocks et al 1989b); quantum phenomena, via the Ricatti equation (Stocks et al 1989a, 1993a); relaxation near a predicted noise-induced transition, falsifying (Jackson et al 1989) an earlier theory and leading to a deeper understanding encompassing coloured noise (Mannella et al 1990); supernarrow spectral peaks near a kinetic phase transition (Dykman et al 1990e); velocity spectra for Brownian motion driven by coloured noise in a periodic potential (Igarashi et al 1992); and noise-induced chaos in the Lorenz model (Fedchenia et al 1992). Earlier reviews of the application of analogue techniques to problems in stochastic nonlinear dynamics include those by Fronzoni (1989), McClintock and Moss (1989) and Mannella and McClintock (1990).

We start, in section 2, by describing the basis of the technique, with the intention of providing sufficient detail to enable other scientists to apply it in practice. In sections 3-7 we discuss the use of analogue simulation to illuminate the understanding of several physical phenomena selected, in part, to demonstrate the ease with which the technique may be applied to a wide diversity of challenging problems. Finally, in section 8 , we summarize the present status of the work and offer some speculative comments about future directions.

\section{Technical details}

The aim is to build an electronic model of the system to be investigated, and then to study its properties, usually while being driven by randon fluctuations (noise) and/or other external forces. In this section, we sketch the basic principles of circuit design, discuss noise generators and outline the steps to be taken in analysing the signal(s) coming from the circuit model.

\subsection{Circuit design}

The work-horse of modern analogue circuit design is the operational amplifier. For present purposes, it enables most of the arithmetic operations needed to model the equations of 
interest to be effected in a very convenient and economical fashion. An excellent, detailed, account of the use of operational amplifiers and other integrated circuits (ICs) will be found in the book by Peyton and Walsh (1993). Here, just for completeness, we summarize very briefly the simple circuit configurations that seem to be used most frequently in modelling operations.

- Addition. Voltages may be added using the general arrangement sketched in figure 1(a). This example is for three input voltages $V_{1}, V_{2}$ and $V_{3}$ but, in principle, any number may be used; note that the output is inverted

$$
V_{\text {out }}=-\left(\frac{R_{F}}{R_{1}} V_{1}+\frac{R_{F}}{R_{2}} V_{2}+\frac{R_{F}}{R_{3}} V_{3}\right) .
$$

Non-inverting addition is also easy to effect (Peyton and Walsh 1993), through use of the positive input point, but it is somewhat less flexible in practice; adjustment of the sign is in any case easy when analogue multipliers (see later) are in use.

- Subtraction. Two voltages $V_{1}$ and $V_{2}$ can readily be subtracted by use of the arrangement in figure 1(b), for which

$$
V_{\text {out }}=\left(\frac{R_{2}}{R_{1}}\right)\left(V_{1}-V_{2}\right) .
$$

- Multiplication or division by a constant factor can be implemented simply by choosing appropriate ratios of input and feedback resistors, $R_{F} / R_{1}$ or $R_{2} / R_{1}$, in the circuits shown in figures $1(\mathrm{a})$ and (b), respectively.

- Integration may be effected by use of the Miller integrator of figure 1(c), for which

$$
C \frac{\mathrm{d} V_{\text {out }}}{\mathrm{d} t}+\frac{V_{\text {in }}}{R}=0
$$

so that the output is

$$
V_{\text {out }}=-\frac{1}{R C} \int_{0}^{t} V_{\text {in }} \mathrm{d} t
$$

with the time constant $R C$.

- Multiplication of two voltages is most conveniently performed by use of an analogue multiplier IC, for example the Analog Devices AD534 or the Burr-Brown MPY-100. The operation of these differential input ICs in multiplier mode can be as sketched in figure 1(d), for which

$$
V_{\text {out }}=\frac{1}{\Phi}\left(V_{x 1}-V_{x 2}\right)\left(V_{y 1}-V_{y 2}\right)+\left(V_{z 1}-V_{z 2}\right)
$$

where the scale factor $\Phi$ is used to prevent the product voltage exceeding the specified maximum. The default value $\Phi=10$ can be adjusted to suit particular situations. In what follows, multipliers will often be shown in circuit block diagrams as though their inputs were single-ended (rather than differential). In such cases, the appropriate input terminals are chosen so as to provide the desired sign of product at the output, and the other terminal of each pair is earthed.

- Division of two voltages can be performed, using the same multiplier ICs, but connecting the output back into one of the inputs as shown in figure 1(e), in which case

$$
V_{\text {out }}=\frac{\Phi\left(V_{z 2}-V_{z 1}\right)}{\left(V_{x 1}-V_{x 2}\right)}+V_{y 1} \text {. }
$$

- Trigonometric functions can be implemented by use of, for example the Analog Devices AD639 universal trigonometric converter IC, which can be connected to transform 
(a)
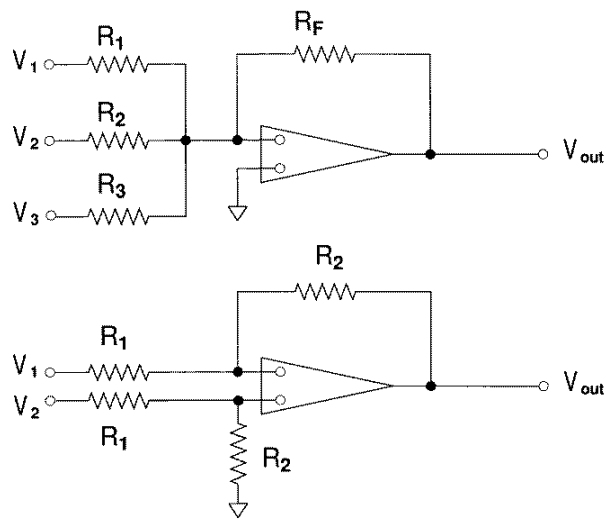

(c)

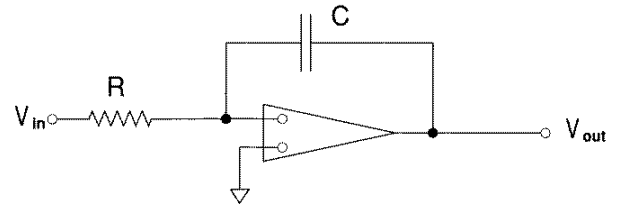

(d)

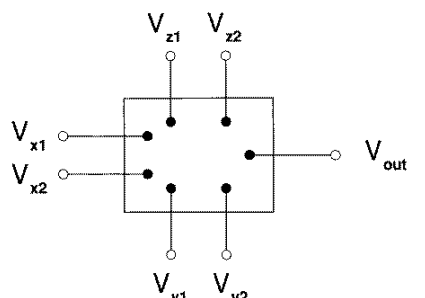

(e)

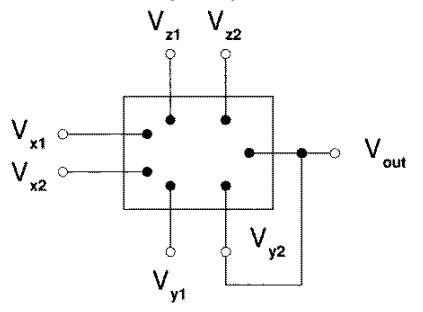

(f)

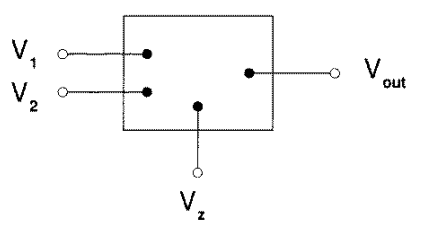

(g)

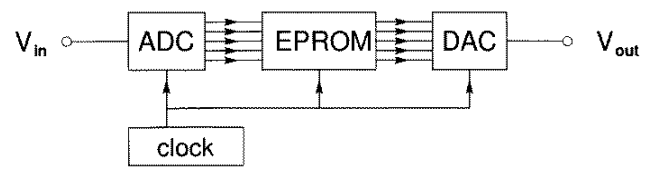

Figure 1. Mathematical operations with analogue electronic circuits: (a) addition; (b) subtraction; (c) integration; (d) multiplication of two voltages; (e) division of two voltages; (f) generation of trigonometric functions; (g) generation of general functions. The respective transformation equations are given in the text. 
the input voltage into its sine, cosine, tangent, cosecant, secant, cotangent or their inverse functions. For example, it can be connected as indicated in figure 1(f), where the output voltage is

$$
V_{\text {out }}=10 \sin \left[50\left(V_{1}-V_{2}\right)\right]+V_{3} .
$$

Here, $V_{1}, V_{2}$ and $V_{3}$ are in volts and the argument of the sine is in degrees. Obviously, a cosine can be generated by setting either $50 V_{1}$ or $50 V_{2}$ to $90^{\circ}$. A range of $\pm 500^{\circ}$ is accommodated, but can effectively be extended where necessary by use of an analogue multiplier IC to generate the double angle (see section 4.4 later).

- General functions can be created by use of a hybrid analogue/digital device as sketched in figure $1(\mathrm{~g})$. The required function is held in the erasable programmable readonly memory (EPROM) as a look-up table. The input voltage is digitized by the analogue to digital converter (ADC) and used to look up the corresponding number in the EPROM, which is then converted to a voltage at the output by the digital to analogue converter (DAC). Devices of this kind have been used, for example, to create trigonometric functions (Vogel et al 1987b) (before the corresponding converter ICs had become available), and for creating the potential corresponding to a one-dimensional quantum-mechanical binary alloy (Stocks et al 1993a). The period of the clock used to drive such a device must, of course, be very much shorter than all characteristic times in the system under study.

In assembling these circuit elements to model a given equation or system of equations, there are several points to be borne in mind.

(1) To minimize extraneous noise introduced by the circuit itself, the design should be such as to minimize the number of active components, for example operational amplifiers or analogue multipliers, as discussed by Fronzoni (1989).

(2) Care must, of course, be taken to ensure that the voltage limits for the various components are not exceeded. Thus, a typical circuit must operate within manufacturers' specifications not only under equilibrium conditions, but also when external forces are driving it far from its stationary state: even the largest excursions to be expected must remain within design specifications, and not only at the output, but also at all intermediate points too.

(3) At the same time, care must also be taken to ensure that the signal is, at all points in the circuit, larger (preferably much larger) than the background noise and drift produced by the circuit itself. This requirement, taken with the preceding one, means that the dynamic range of the analogue technique is necessarily limited: the maximum tolerable voltage swings are typically $\pm 10 \mathrm{~V}$, and intrinsic noise is typically $\pm 0.5 \mathrm{mV}$ peak-to-peak, giving an effective dynamic range of $2 \times 10^{4}$. With careful circuit design, this is more than sufficient for most purposes.

(4) To speed up data acquisition, it is normal to scale time in such a way that time in the model effectively runs much faster than real time. In doing so, care must be taken to remain within the allowed frequency limits of the active components; because the bandwidth is related to amplitude, it is usually best to compare expected and allowable slew rates. Again, the validity of operation should be checked at all intermediate points, and not just at the output.

(5) To reduce the effect of stray capacitance, connections should be made as short and direct as possible, and the circuit layout planned accordingly. Where a circuit model is likely to be needed for many different applications, or where particular stability is needed, it is worth designing and fabricating a special printed circuit board (PCB). In most cases, however, a simple mounting board into which the components can be inserted (with pressure contacts) is sufficient, and is of course convenient in that modifications are fast and easy. 
(6) It is usually best to choose resistor values within (or not too far outside) the range $10-100 \mathrm{k} \Omega$, and capacitance values of at least $100 \mathrm{pF}$ in view of the typical stray capacitance of a few $\mathrm{pF}$.

Optimization of the design requires that all of the above criteria should be taken into account, but some degree of compromise between them is usually necessary in practice. Minimization of the number of active components can often be achieved by making judicious combinations (in single circuit elements) of the separate arithmetic operations described above.

To see how a circuit model can be designed in practice, we now consider a particular example: the underdamped single-well Duffing oscillator used (Dykman et al 1994c) for experiments on supernarrow spectral peaks and high-frequency stochastic resonance in a system with co-existing periodic attractors (see section 4.3 later). The equation to be modelled is

$$
\ddot{x}+2 \Gamma \dot{x}+\omega_{0}^{2} x+\gamma x^{3}=F \cos \omega_{F} t+f(t)
$$

where the oscillator is driven by a periodic force of amplitude $F$, frequency $\omega_{F}$ and $f(t)$ is the zero-mean white Gaussian noise of intensity $D$ such that

$$
\langle f(t)\rangle=0 \quad\langle f(t) f(s)\rangle=4 \Gamma D \delta(t-s) .
$$

The circuit used to model (8) is shown in figure 2: two integrators are needed because of the inertial term. The periodic force in the dashed box is set to zero for present purposes (but it will be needed in section 4.3 in the discussion of high-frequency stochastic resonance in the same system). We use primes to distinguish times and frequencies in the circuit (in units of $\mathrm{s}$ and $\mathrm{Hz}$ ) from the corresponding dimensionless times and frequencies that appear in equation (8). To understand the relationships between quantities in the circuit and in (8), we sum the currents at point $\mathrm{A}$, and those at point $\mathrm{B}$, and we equate them to zero in each case (using Kirchhoff's law and the fact that the input impedance of an operational amplifier is effectively infinite). For point A

$$
\frac{V_{1}}{R_{3}}+\frac{f^{\prime}\left(t^{\prime}\right)}{R_{1}}+\frac{F^{\prime}}{R_{2}} \cos \left(\omega_{F}^{\prime} t^{\prime}\right)-\frac{V_{2}^{3}}{20 R_{6}}-\frac{V_{2}}{R_{5}}+C_{1} \frac{\mathrm{d} V_{1}}{\mathrm{~d} t^{\prime}}=0
$$

and at point $\mathrm{B}$

$$
\frac{V_{1}}{R_{4}}+C_{2} \frac{\mathrm{d} V_{2}}{\mathrm{~d} t^{\prime}}=0 .
$$

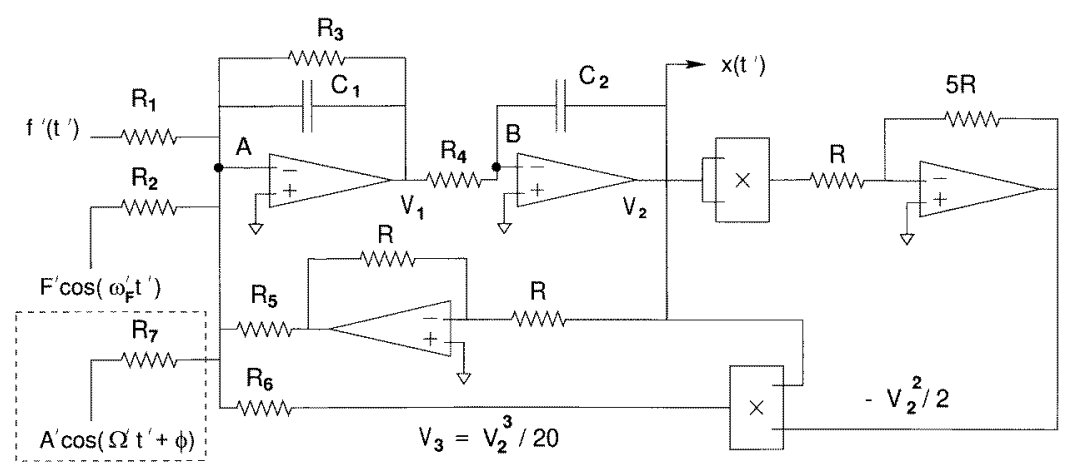

Figure 2. A block diagram of an analogue electronic circuit modelling an underdamped singlewell Duffing oscillator (8) (Dykman et al 1994c). 
Using (11) to substitute for $V_{1}$ in (10), we obtain

$C_{1} R_{4} C_{2} \frac{\mathrm{d}^{2} V_{2}}{\mathrm{~d} t^{\prime 2}}+\frac{R_{4} C_{2}}{R_{3}} \frac{\mathrm{d} V_{2}}{\mathrm{~d} t^{\prime}}+\frac{V_{2}}{R_{5}}+\frac{V_{2}^{3}}{20 R_{6}}-\frac{f^{\prime}\left(t^{\prime}\right)}{R_{1}}-\frac{F^{\prime}}{R_{2}} \cos \left(\omega_{F}^{\prime} t^{\prime}\right)=0$.

The component values used in the circuit were

$$
\begin{aligned}
& R_{1}=R_{4}=R_{5}=2 R_{6}=\frac{R_{2}}{10}=\frac{R_{0}}{10}=100 \mathrm{k} \Omega \\
& R_{3}=2.5 \mathrm{M} \Omega \\
& C_{1}=C_{2}=1 \mathrm{nF} .
\end{aligned}
$$

Thus equation (12) may be written

$$
\tau^{2} \frac{\mathrm{d}^{2} V_{2}}{\mathrm{~d} t^{\prime 2}}+2 \Gamma \tau \frac{\mathrm{d} V_{2}}{\mathrm{~d} t^{\prime}}+V_{2}+\frac{V_{2}^{3}}{10}=\frac{F^{\prime}}{10} \cos \left(\omega_{F}^{\prime} t^{\prime}\right)+f^{\prime}\left(t^{\prime}\right)
$$

where

$$
\tau=R_{1} C_{1}=R_{4} C_{2} \quad \Gamma=\frac{R_{1}}{2 R_{3}} .
$$

It can be seen, therefore, that with the transformations

$V_{2} \rightarrow x \quad t^{\prime} \rightarrow \tau t \quad \omega_{F}^{\prime} \rightarrow \omega_{F} / \tau \quad F^{\prime} \rightarrow 10 F \quad f^{\prime}\left(t^{\prime}\right) \rightarrow f(t)$

(13) goes over into (8) with $\omega_{0}=1$ and $\gamma=0.1$. Note that the multiplication of $V_{2}^{2}$ by $\times 5$ (figure 2) is used to prevent the $V_{2}^{3}$ term being too small compared to background noise and voltage offsets in the circuit, following the additional scaling by 0.1 in the second multiplier. The relatively large value of $R_{3}=2.5 \mathrm{M} \Omega$ was used to obtain a small value of the damping constant $\Gamma$. Under these circumstances, it is better to measure quantities like $\Gamma$ and $\omega_{0}$ for the completed circuit (McClintock et al 1993), treating it as an experimental object in its own right, rather than just calculating them from component values; the measured and calculated values typically agree to within $\pm 10 \%$.

\subsection{Noise generators}

Experiments are often undertaken to model systems driven by white noise. In reality, of course, white noise-with zero correlation time and thus a power spectrum that remains flat up to infinite frequency-is an idealization. Noise in real physical systems always has a finite correlation time, and correspondingly a roll-off in the power spectrum above some characteristic frequency. Provided that the correlation time of the noise is much smaller (e.g. by a factor of 30) than all characteristic times (the vibration period(s) for an underdamped system, or the relaxation time(s) for an overdamped one), the noise can be considered quasi-white and its effects will be indistinguishable from those of white noise with the same intensity.

A number of commercial noise generators have been used for experiments in stochastic nonlinear dynamics. These have included the Quan-Tech model 420 (no longer manufactured) and, more recently, the Wandel and Goltermann $\dagger$ model RG1. The latter produces Gaussian noise with a power spectrum that is flat to within $\pm 0.5 \mathrm{~dB}$ up to $108 \mathrm{kHz}$; above this frequency, there is a very fast roll-off. It possesses the advantage that the output is genuinely random, with an essentially infinite repetition period. At the same time it has the disadvantages that there is significant 'sag' of the power spectrum (though within specification) in the intermediate frequency range and that it is relatively expensive.

$\dagger$ Wandel u. Goltermann, 7410 Reutlingen, Germany. 
For many purposes, however, a much cheaper and simpler 'home-made' noise generator will suffice which, in some respects, is actually superior. This is the device used and described by Faetti et al $(1984,1985)$ and Fronzoni (1989). It is based on the generation of a pseudo-random sequence by a linear-feedback shift register (Golomb 1967); the dichotomous (two-state) output is then filtered through a Miller integrator to produce (Rice 1944) the Ornstein-Uhlenbeck (i.e. exponentially correlated) noise. In its original form, the dichotomous noise suffered from asymmetry in that the times spent in the upper and lower states were systematically different on account of the OR-feedback being used (Tomlinson and Galvin 1975). Consequently, the distribution function of the approximately Gaussian noise created after passage through the filter suffered from significant skewness. Faetti et al (1985) showed that this problem could be overcome by randomly inverting the sign of the feedback voltage between EX-OR and EX-NOR. A version of this device (figure 3) used at Lancaster (Casademunt et al 1989c) employs the output from two different stages of the same 17-stage feedback shift register (SR1) as feedback inverters for two separate 41-stage (Freeman 1988) shift registers (SR2 and SR3). In this way, two independent pseudo-random pulse sequences are obtained which, after filtration, provide uncorrelated pseudo-white noise sources. With a clock frequency of $\sim 4 \mathrm{MHz}$, and the filter time constants set to give a cut-off above $40 \mathrm{kHz}$, the distribution functions at the output are Gaussian to more than \pm 4 standard deviations and the repetition time of the pseudo-random time sequences is $\sim 6.5$ days. There is no detectable 'sag' in the power spectrum. It is essential to remember that these noise generators should not be used for experiments whose duration exceeds the repetition time; in all other respects, however, they have been found entirely satisfactory.

The root-mean-square (RMS) amplitude of the Ornstein-Uhlenbeck noise being applied to a circuit model can conveniently be measured with a true RMS to DC converter such as

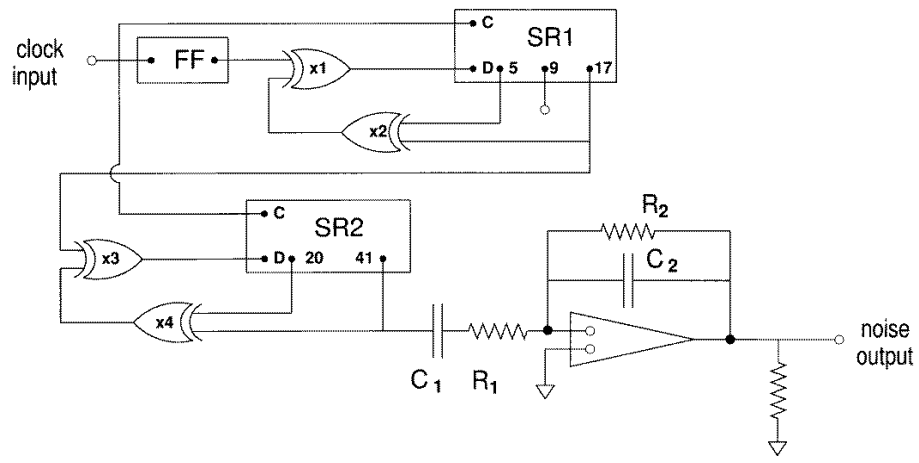

Figure 3. A block diagram of the linear-feedback shift-register digital noise generators used at Lancaster, based on a design developed in the University of Pisa (Faetti et al 1984, 1985; Fronzoni 1989). Pseudo-random dichotomous (two-level) pulse sequences are generated by the 41-stage feedback shift-register SR2. The high-cut filter converts this dichotomous noise to an output that is Gaussian and exponentially correlated, with a correlation time $R_{2} C_{2}$. The first feedback shift register SR1 pseudo-randomly inverts the sign of the feedback in SR2, thereby eliminating skewness of the distribution. An additional 41-stage feedback shift register SR3 (not shown), taking its inverting input from stage 9 of SR1 but otherwise connected exactly like SR2, provides a second independent noise source. FF is a flip-flop that divides the $4 \mathrm{MHz}$ clock frequency by a factor of 32 , and $\mathrm{X} 1-\mathrm{X} 4$ are exclusive-OR gates. The low-frequency cut-off, determined by $R_{1} C_{1}$, is fixed at $\sim 1 \mathrm{~Hz}$, and the high-frequency cut-off is adjustable. The output noise finally passes through a variable-gain operational amplifier (not shown) before being applied to an experiment. 
the Analog Devices AD536A IC. It is then necessary to relate this value (in volts) to the intensity $D$ of white noise appearing in model equations such as (8) and (9). To do so, we note that Ornstein-Uhlenbeck noise is exponentially correlated, with

$$
\langle f(t) f(s)\rangle=\frac{2 \Gamma D}{\tau_{N}} \mathrm{e}^{-|t-s| / \tau_{N}}
$$

where $\tau_{N}$ is the correlation time. Thus, the mean-square noise amplitude is

$$
\left\langle f^{2}(t)\right\rangle=\frac{2 \Gamma D}{\tau_{N}} .
$$

But the circuit operates in scaled time, so that

$$
\tau_{N}=\frac{\tau_{\mathrm{OU}}}{\tau}
$$

where $\tau_{\mathrm{OU}}$ is the measured or calculated correlation time of the noise (in seconds), and $\tau$ is the time scaling of the model, given by equation (15) in the example in section 2.1. Hence,

$$
D=\left(\frac{\tau_{\mathrm{OU}}}{2 \Gamma \tau}\right)\left\langle f^{2}(t)\right\rangle .
$$

Here, $\left\langle f^{2}(t)\right\rangle$ can be measured directly. Values of all the other parameters are also known, because they can be calculated from the component values or measured directly. Thus (19) provides the required connection between $D$ and the RMS noise voltage measured by the true RMS to DC converter IC.

Note that the fast roll-off of some commercial noise generators, such as the Wandel and Goltermann model mentioned above, means that they do not produce OrnsteinUhlenbeck noise as they stand. Passage of the output through a Miller integrator with a time constant of at least $\sim 1 \mathrm{~ms}$ (for a roll-off at $100 \mathrm{kHz}$ ) will perform the necessary conversion. If the integrating filter has a smaller time constant than this, however, misleading results can be obtained: the output may seem to correspond to OrnsteinUhlenbeck noise, but it is deficient in high-frequency components, so there is likely to be poor agreement between the apparent $D$ measured for the model and the $D$ that appears in the equations.

\subsection{Signal acquisition and analysis}

Analysis of the behaviour of the circuit model usually involves two main stages: digitization of the analogue signal $x(t)\left(V_{2}(t)\right.$ in the above example); and then processing of the resultant digital time series to extract the particular information required, which is often in the form of a statistical distribution.

The first 12 years of stochastic/nonlinear research by the Lancaster group, including a large proportion of the work described below, depended on the use of Nicolet data processors (models NIC-80, NIC-1180 and NIC-1280) both to digitize and analyse the signals. These were quite remarkable instruments for their time, but are now obsolete and are currently being replaced by PC-based equivalent systems with enhanced capabilities.

There is now a very wide range of ADC cards available for operation in PCs. From time to time, helpful reviews are published (see, for example, Barton 1991, Rand 1994) which may help in the selection of the most suitable device for a particular application. Amongst other factors, it will be necessary to consider: the number of bits required for the digitized signal (12-bits has been standard, but 16-bit ADCs are now becoming more widely available); the minimum sample interval (values below $1 \mu$ s are not normally needed, and $10 \mu \mathrm{s}$ is short enough for most purposes), the speed limits being largely 
determined by those of the standard ICs used for the models, and the fact that the latter usually involve open mounting boards without optimized high-speed component layout; the maximum block size (number of consecutive data samples without gaps) required, which in some cases will involve continuous acquisition (no data gaps); and whether or not a digital signal processing (DSP) or other type of co-processor is required on the card. If fast data acquisition without gaps-coupled with intensive processing-is needed, a coprocessor is likely to be essential. The advantage is that most of the data processing can then take place on the card itself, with relatively infrequent transfers of input data and ensemble-averaged distribution to the main memory. The PC's central processing unit (CPU) thus remains free to operate displays of the input and averaged data, interact with the user, and service the 'housekeeping' needs of the operating system. If the required distributions are of a conventional kind (e.g. power spectra), a DSP co-processor is likely to be best. For creating less common types of distribution (see later), a more flexible type of co-processor is likely to be preferable. The systems currently in use and under development at Lancaster are based on the Microstar† model DAP 3200a/415 (12-bit) and DAP 3216/415 (16-bit) ADCs, each of which incorporates an on-board $100 \mathrm{MHz}$ Intel 486DX co-processor.

In practice, most of the experiments require purpose-designed software. Thus, although single-variable probability distributions or power spectra may be available as parts of commercial packages, the numerous other types of distribution that are of interest will in most cases need to be programmed specially. These will include (see later), for example, prehistory probability densities, time-evolving distributions in one or more variables, first passage time distributions, sojourn time distributions for two-variable systems with two or more attractors, provision for measuring a variety of distributions related to the centre-ofmotion of a system driven by quasimonochromatic noise (QMN), and many others. Even for seemingly standard applications, minor but essential variations in the required processing may necessitate coding the software specially, for example the need to subtract a timedomain ensemble average of the input before processing each block to calculate power spectra. In any case, to optimize the rate of data acquisition/processing, it will often be necessary to design a system that will exploit a co-processor to do most of the work as described above, which will not usually be possible with a standard package. The particular advantage of the Microstar co-processor is that it may be programmed using the same compilers and support software that are already in use with the host PC, which simplifies the task considerably.

\section{Large rare fluctuations}

Perhaps one of the most convincing demonstrations of how simple analogue electronic techniques can be used to develop a new experimental approach, and to obtain direct experimental insight into long-standing problems of fundamental importance, is through its application to the investigation of large fluctuations.

\subsection{The concept of large fluctuations}

The concept of large fluctuations was discussed by Boltzmann (1904). In a great many cases, the characteristic fluctuation intensity $D$ is small, and large fluctuation events, in

$\dagger$ Microstar Laboratories Inc, 2265 116th Avenue NE, Bellevue, WA 98004, USA; and see http://www.mstarlabs.com/. The UK distributor is: Amplicon Liveline Ltd, Centenary Industrial Estate, Hollindean Road, Brighton BN2 4AW. 
which the system moves far away from its stable state(s) in the phase space, are very rare. Although infrequent, large fluctuations can be responsible for big qualitative changes in a system, and they therefore play a crucial role in numerous important phenomena, for example nucleation at phase transitions, chemical reactions, mutations in DNA sequences, protein transport in biological cells and failures of electronic devices.

In many cases the fluctuating systems of interest are far from thermal equilibrium. Examples include lasers, pattern forming systems (Cross and Hohenberg 1993), trapped electrons which display bistability and switching in a strong periodic field (Gabrielse et al 1985, Dehmelt 1990, Tan and Gabrielse 1993) and spatially periodic systems (ratchets) which display a unidirectional current when driven away from thermal equilibrium (Magnasco 1993, Astumian and Bier 1994, Millonas and Dykman 1994, Prost et al 1994, Doering et al 1994, Leibler 1994, Millonas 1995, Hondou and Sawada 1995, Marchesoni 1996, Dykman et al 1997a). Other examples are discussed in section 4 in the context of stochastic resonance.

The analysis of large fluctuations requires the solution of two closely interrelated problems. The first is the evaluation of the probability density $\rho(\boldsymbol{x})$ for a system to occupy a state $\boldsymbol{x}$ far from the stable state $\boldsymbol{x}_{\mathrm{st}}$ in the phase space. In the stationary regime, the function $\rho(\boldsymbol{x})$ is independent of time and has a maximum at $\boldsymbol{x}_{\mathrm{st}}$. The tails of $\rho(\boldsymbol{x})$ are determined by the probabilities of large fluctuations.

The other problem is that of the fluctuational paths along which the system moves when a large fluctuation occurs. The distribution of fluctuational paths is a fundamental characteristic of the fluctuation dynamics, and its understanding paves the way to developing techniques for controlling fluctuations. Its importance for gaining insight into the physics of fluctuations from a dynamical perspective was recognized more than 40 years ago by Onsager and Machlup (1953). A theoretical understanding, and basic techniques for treating the problem, have been developed since that time; but it was not until recently (Dykman et al 1992c) that a method for observing the distribution of fluctuational paths for large fluctuations was proposed and implemented by analogue simulation.

A simple qualitative idea behind the theory of large fluctuations in noise-driven systems is that such fluctuations result from large outbursts of noise $f(t)$ that push the system far from the attractor. The probabilities of large outbursts are small, and the value of the probability density $\rho\left(\boldsymbol{x}_{f}\right)$ for a given remote $\boldsymbol{x}_{f}$ will actually be determined by the probability of the most probable outburst of those capable of bringing the system to $\boldsymbol{x}_{f}$. This particular realization of noise is just the optimal fluctuational force $f_{\text {opt }}(t)$ for the given $\boldsymbol{x}_{f}$. Because a realization (a path) of noise $\boldsymbol{f}(t)$ results in the corresponding realization of the dynamical variable $\boldsymbol{x}(t)$ (Feynman and Hibbs 1965), there also exists an optimal path $\boldsymbol{x}_{\mathrm{opt}}\left(t ; \boldsymbol{x}_{f}\right)$ along which the system arrives at $\boldsymbol{x}_{f}$, with overwhelming probability. From a different perspective, optimal paths $\boldsymbol{x}_{\mathrm{opt}}\left(t ; \boldsymbol{x}_{f}\right)$ were first described for nonlinear non-equilibrium Markov systems by Ventcel' and Freidlin (1970). (Note that 'Ventcel" is often, and more correctly, spelled Wentzell in the literature.) Using another approach, the analysis of the tails of the distribution was also performed by Graham (1973), whereas the approach described above was suggested independently by Dykman and Krivoglaz (1979) in the context of escape from a metastable state. This approach is not limited to Markov systems (Dykman and Krivoglaz 1984, Dykman 1990). For systems driven by OrnsteinUhlenbeck noise, optimal paths were discussed by Luciani and Verga (1987, 1988), Tsironis and Grigolini (1988), Bray and McKane (1989), McKane (1989), Wio et al (1989), McKane et al (1990), Bray et al (1990) and Luckock and McKane (1990), whereas an equivalent eikonal formulation was developed by Kłosek-Dygas et al (1988a, b, 1989). The general case of Gaussian noise was discussed by Dykman (1990) and Dykman and Smelyanskiy 
(1998a) (see section 6) and by Einchcomb and McKane (1994, 1995). Reviews of related work on fluctuations in systems driven by coloured noise were given by Lindenberg et al (1989b) and by Dykman and Lindenberg (1994).

The major qualitative result of the theory of optimal paths in classical systems is that these paths are real physical trajectories (see Dykman 1990). Although the arrival at a given state $\boldsymbol{x}_{f}$ is the result of a fluctuation, the motion on the way to this state is essentially deterministic. It occurs in real time, in the space of the dynamical variables of the system. In other words, if we find a system in a given state far from an attractor, and we know that the system had been fluctuating about that attractor for a time much longer than either the relaxation time or the correlation time of the fluctuating bath (or the driving noise), then we can tell with high probability how the system moved to this state. To some extent this statement is counterintuitive, as our daily experience tells us (Lebowitz 1993) that, if we find the system far away from its stable equilibrium, it means that it had been prepared even further away from equilibrium, and we just happened to find it on its way to equilibrium. However, as shown by Einstein (1910), large fluctuations may still happen even in the equilibrium state, at the expense of a (very unlikely, yet possible) decrease in entropy. The optimal path concept shows that this decrease develops in time in a certain optimal way.

Optimal fluctuational paths are a counterpart of the relaxational paths in the absence of fluctuations, and they have also much in common with rays in geometrical optics and with WKB trajectories in quantum mechanics. Optimal fluctuational paths and rays both represent the most likely ways to be followed. Formally, they are the paths that provide extrema to certain functionals (the least action principle). The pattern of rays in optics displays singularities (Berry 1976, 1977), and therefore similar singularities might be expected in the pattern of optimal paths. And indeed, they seemed to appear when the appropriate equations of motion were solved formally (Jauslin 1987b, Day 1987, Chinarov et al 1993, Maier and Stein 1993a, b, 1996b). It gave rise to concern about the applicability of the method near singularities (Risken 1993). However, it was soon realized that the observable singularities of the pattern of optimal fluctuational paths may be different from those in geometrical optics (Jauslin 1987b, Dykman 1990). It was shown recently that, for Markov systems, the singularities can be understood and classified using topological arguments (Dykman et al 1994d, Smelyanksiy et al 1997a). Nevertheless, the very prediction of an onset of singularities is a challenge to experiment.

The notion of the optimal fluctuational path being a counterpart of the relaxational path in the absence of fluctuations has long been a major tool for tackling fluctuation dynamics. Yet, until recently, there was no direct experimental evidence in support of the idea. Arguably, therefore, the theory was based on an act of faith. One experimental problem was that, if the noise intensity is too small, it is hard to obtain good statistics for arrivals of the system at a remote state whereas, if the noise intensity is too large so that the required fluctuations happen often, the notion of the optimal path becomes irrelevant. Another problem was that experiments on optimal fluctuational paths could not be undertaken systematically until the appropriate statistical quantity, the prehistory probability distribution (Dykman et al 1992c), had been introduced.

In this situation, analogue electronic models have provided an ideal opportunity for the development of new experimental techniques, based on measurements of the prehistory probability distribution. As we shall see, they led to the first direct experimental observations of the dynamics of large fluctuations in noise-driven systems. Note that similar behaviour is to be expected not only of noise-driven systems, but of other fluctuating systems as well. 


\subsection{Prehistory probability distribution}

To investigate the dynamics of large fluctuations in the stationary regime, one may adopt an approach (Dykman et al 1992c) in which one accumulates the information about all arrivals of the system in the close vicinity of a chosen state $\boldsymbol{x}_{f}$. In the experiments the state of the system is monitored continuously. Two such events, where the trajectory of a one-variable system passed through $\boldsymbol{x}_{f}$, are shown in figure 4 . The interesting region of the path - the fluctuational part $f$ coming to $\boldsymbol{x}_{f}$-is then stored. An ensemble-average of such trajectories (see later), built up over a period of continuous monitoring (typically weeks), creates the prehistory probability distribution $p_{h}\left(\boldsymbol{x}, t ; \boldsymbol{x}_{f}, t_{f}\right)$ (Dykman et al 1992c). If a point $\boldsymbol{x}_{f}$ lies far from the attractor, so that the stationary probability density $\rho\left(\boldsymbol{x}_{f}\right)$ is small, the time intervals between successive passages of $\boldsymbol{x}_{f}$ will be large; they will considerably exceed both the characteristic relaxation time of the system, $\tau_{\mathrm{r}}$, and the noise correlation time, $\tau_{\mathrm{c}}$. The arrivals of the system at $\boldsymbol{x}_{f}$ are, therefore, mutually uncorrelated. Since the moment of observation $t_{f}$ is the only instant of time singled out under stationary conditions, the prehistory probability distribution can be consistently defined as the probability density $p_{h}\left(\boldsymbol{x}, t ; \boldsymbol{x}_{f} t_{f}\right) \equiv \rho\left(\boldsymbol{x}_{i}, t_{i} ; \boldsymbol{x}, t ; \boldsymbol{x}_{f}, t_{f}\right)$ of the system being at $x$ at time $t$ if it was at $\boldsymbol{x}_{f}$ at time $t_{f}$ (where $t_{f}>t$ ), with $t_{i} \rightarrow-\infty$, and $\boldsymbol{x}_{i}$ close to the attractor $\boldsymbol{x}_{\mathrm{eq}}$.

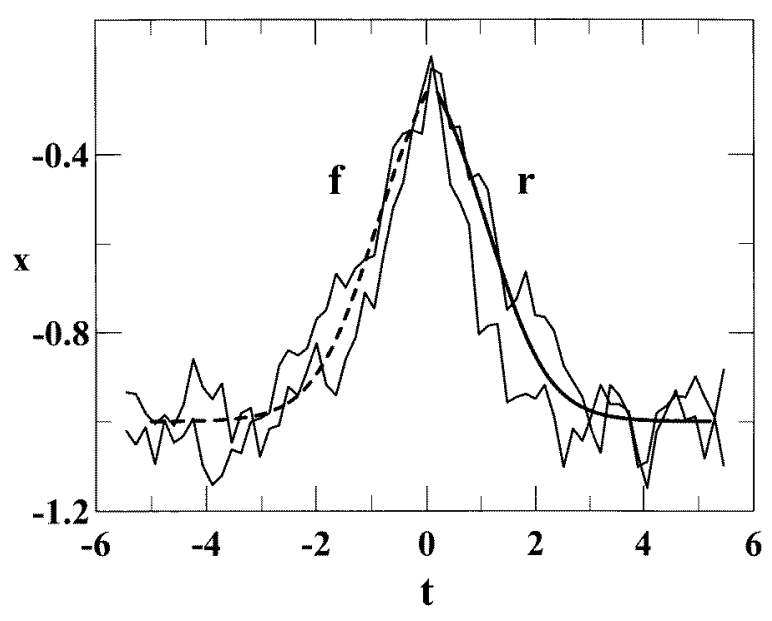

Figure 4. Fluctuational behaviour measured and calculated for a simple one-dimensional model equilibrium system: the double-well Duffing oscillator with $K(x)=x-x^{3}$, for $D=0.014$ in (21), (32). Two typical fluctuations (jagged lines) from the stable state at $S=-1$ to the remote state $x_{f}=-0.1$ and back again are compared with the calculated deterministic relaxational path from $x_{f}$ to $S$ (full, smooth, curve) and the calculated time-reversed relaxational path from $S$ to $x_{f}$ (dashed curve) (Luchinsky 1997).

We stress that $p_{h}\left(\boldsymbol{x}, t ; \boldsymbol{x}_{f}, t_{f}\right)$ is not a standard two-time transition probability: it is given by a ratio of the transition probability $\rho\left(\boldsymbol{x}_{f}, t_{f} ; \boldsymbol{x}, t \mid \boldsymbol{x}_{i}, t_{i}\right)$, (the conditional probability density for a system placed initially at $\boldsymbol{x}_{i}$ to pass through the states $\boldsymbol{x}$ and $\boldsymbol{x}_{f}$ at the instants $t$ and $t_{f}$, respectively), to the two-time transition probability $\rho\left(\boldsymbol{x}_{f}, t_{f} \mid \boldsymbol{x}_{i}, t_{i}\right)$, with the limit being taken in which the initial instant $t_{i}$ goes to $-\infty$ (however, in the case of fluctuations from a metastable state we assume that the time interval $t_{f}-t_{i}$ is less than the lifetime of the state). A similar ratio of transition probabilities was considered for Markov processes by Schulman (1991) in order to clarify discussions about the relationship between the thermodynamic and cosmological arrows of time. 
In the original paper (Dykman et al 1992c) the distribution $p_{h}\left(\boldsymbol{x}, t ; \boldsymbol{x}_{f}, t_{f}\right)$ was introduced in such a form that the definition applies both to Markov and non-Markov systems, as the distribution is expressed in terms of the probability density functional $\mathcal{P}[\boldsymbol{x}(t)]$ of the paths $\boldsymbol{x}(t)$ of a noise-driven system,

$$
\begin{aligned}
& p_{h}\left(\boldsymbol{x}, t ; \boldsymbol{x}_{f}, t_{f}\right)=\rho\left(\boldsymbol{x}_{f}, t_{f} ; \boldsymbol{x}, t \mid \boldsymbol{x}_{i}, t_{i}\right) / \rho\left(\boldsymbol{x}_{f}, t_{f} \mid \boldsymbol{x}_{i}, t_{i}\right) \\
& p_{h}\left(\boldsymbol{x}, t ; \boldsymbol{x}_{f}, t_{f}\right)=\int_{x\left(t_{i}\right) \approx x_{\mathrm{cq}}\left(t_{i}\right)}^{x\left(t_{f}\right)=x_{f}} \mathcal{D} \boldsymbol{x}\left(t^{\prime}\right) \delta(\boldsymbol{x}(t)-\boldsymbol{x}) \mathcal{P}[\boldsymbol{x}(t)] \\
& \times\left(\int_{\boldsymbol{x}\left(t_{i}\right) \approx x_{\mathrm{eq}}\left(t_{i}\right)}^{\boldsymbol{x}\left(t_{f}\right)=x_{f}} \mathcal{D} \boldsymbol{x}\left(t^{\prime}\right) \mathcal{P}[\boldsymbol{x}(t)]\right)^{-1} \quad t_{i} \rightarrow-\infty .
\end{aligned}
$$

We note that the initial value of $\boldsymbol{x}\left(t_{i}\right)$ for $t \rightarrow-\infty$ in (20) may in fact be arbitrary, provided $\left(t-t_{i}\right)$ and $\left(t_{f}-t_{i}\right)$ substantially exceed $\tau_{\mathrm{r}}$ and $\tau_{\mathrm{c}}$, so that the system becomes randomized and will have forgotten its initial position before the start of the fluctuation bringing it to $\boldsymbol{x}_{f}$ at $t_{f}$. In this section we will limit our analysis to Markov processes, and we will consider two types of systems: stationary and periodically driven. In the latter case the position of the stable state $\boldsymbol{x}_{\mathrm{eq}}(t)$ in (20) is a periodic function of time, and we will assume that it has the same period as the driving force.

The physical significance of the prehistory probability density $p_{h}\left(\boldsymbol{x}, t ; \boldsymbol{x}_{f}, t_{f}\right)$ follows from the fact that, since the optimal path $\boldsymbol{x}_{\mathrm{opt}}\left(t-t_{f} ; \boldsymbol{x}_{f}\right)$ is the most probable path for reaching $\boldsymbol{x}_{f}$, the function $p_{h}\left(\boldsymbol{x}, t ; \boldsymbol{x}_{f}, t_{f}\right)$ at a given $t-t_{f}$ should have a sharp maximum in $\boldsymbol{x}$ lying on that path, $\boldsymbol{x}=\boldsymbol{x}_{\mathrm{opt}}\left(t-t_{f} ; \boldsymbol{x}_{f}\right)$. Therefore, by investigating the prehistory probability density $p_{h}\left(\boldsymbol{x}, t ; \boldsymbol{x}_{f}, 0\right)$ experimentally one can find not only the optimal paths themselves, but also test immediately the very concepts of the optimal path and the optimal fluctuation, and establish the range of parameters and the area of phase space within which optimal paths are well defined, i.e. where the tube of fluctuational paths around an optimal path is narrow.

A model fluctuating system which is convenient for simulations and which makes it possible to reveal optimal paths and singular features of the distribution of paths is an overdamped Brownian particle driven both by a regular force $\boldsymbol{K}(\boldsymbol{x}, t)$ and by a random force with the same number of independent components as the number of the particle coordinates. We will assume the regular force to be time periodic and/or non-gradient, in general. The Langevin equation of motion of the system is of the form

$$
\begin{aligned}
& \dot{\boldsymbol{x}}=\boldsymbol{K}(\boldsymbol{x}, t)+\boldsymbol{f}(t) \\
& \left\langle f_{i}(t)\right\rangle=0 \quad\left\langle f_{i}\left(t_{1}\right) f_{j}\left(t_{2}\right)\right\rangle=D \delta_{i j} \delta\left(t_{1}-t_{2}\right) .
\end{aligned}
$$

We assume the random force $f(t)$ to be a zero-mean white Gaussian noise, of intensity $D$.

The model (21) describes the fluctuational dynamics of a wide class of macroscopic dissipative systems (Zwanzig 1973, Haken 1975, Kubo et al 1978, Risken 1993). It has numerous counterparts in spatially extended systems, in which case $\boldsymbol{x}$ is a coordinatedependent field (an order parameter), as discussed by Hohenberg and Halperin (1977) and Cross and Hohenberg (1993) (see the papers by Graham and Tél (1990) and Smelyanskiy et al (1997b) and references therein for a discussion of the work on large fluctuations in continuous systems lacking detailed balance).

The probability density functional for a Markovian system (21) can be written as

$$
\mathcal{P}[\boldsymbol{x}(t)]=\exp (-S[\boldsymbol{x}(t)] / D) .
$$

To lowest order in the noise intensity, $S[x(t)]$ takes the form of the action functional for an 
auxiliary dynamical system with the Lagrangian $L(\dot{\boldsymbol{x}}, \boldsymbol{x} ; t)$ (Freidlin and Wentzell 1984):

$$
S[\boldsymbol{x}(t)]=\int_{t_{i}}^{t_{f}} \mathrm{~d} t L(\dot{\boldsymbol{x}}, \boldsymbol{x} ; t) \quad L(\dot{\boldsymbol{x}}, \boldsymbol{x} ; t)=\frac{1}{2}[\dot{\boldsymbol{x}}-\boldsymbol{K}(\boldsymbol{x} ; t)]^{2} .
$$

A simple way to obtain equation (23) is to note that the probability density functional for white noise $f(t)$ is of the form (cf Feynman and Hibbs 1965)

$$
\mathcal{P}_{f}[\boldsymbol{f}(t)]=\exp \left(-\frac{1}{2 D} \int \mathrm{d} t \boldsymbol{f}^{2}(t)\right) .
$$

To find the functional $\mathcal{P}[\boldsymbol{x}(t)]$ for large fluctuations of $\boldsymbol{x}$ one can just express $\boldsymbol{f}(t)$ in (24) in terms of $\boldsymbol{x}(t)$ using the equation of motion (21) (Dykman and Krivoglaz 1979) (the Jacobian of the corresponding transformation gives a correction to $S$ of order $D$ ).

The optimal fluctuational path $\boldsymbol{x}_{\mathrm{opt}}\left(t \mid \boldsymbol{x}_{f}, t_{f}\right)$ along which the system arrives at the point $\boldsymbol{x}_{f}$ at the instant $t_{f}$ is the path that provides the minimum to $S[\boldsymbol{x}(t)]$. It is given by the solution of the variational problem

$$
\begin{aligned}
& \frac{\delta S[\boldsymbol{x}]}{\delta \boldsymbol{x}(t)}=0 \quad \boldsymbol{x}_{\mathrm{opt}}\left(t_{f} \mid \boldsymbol{x}_{f}, t_{f}\right)=\boldsymbol{x}_{f} \\
& \boldsymbol{x}_{\mathrm{opt}}\left(t_{i} \mid \boldsymbol{x}_{f}, t_{f}\right) \rightarrow \boldsymbol{x}_{\mathrm{eq}}\left(t_{i}\right) \quad \text { for } t_{i} \rightarrow-\infty .
\end{aligned}
$$

The variational equation of motion of the auxiliary system (25) can be written in the Lagrangian form, or one can use the equations of motion in the Hamiltonian form. According to (25) the Hamiltonian equations take on the form (Ventcel' and Freidlin 1970)

$$
\begin{aligned}
& \frac{\mathrm{d} \boldsymbol{x}}{\mathrm{d} t}=\frac{\partial H}{\partial \boldsymbol{p}} \quad \frac{\mathrm{d} \boldsymbol{p}}{\mathrm{d} t}=-\frac{\partial H}{\partial \boldsymbol{x}} \quad \frac{\mathrm{d} S}{\mathrm{~d} t}=\frac{1}{2} \boldsymbol{p}^{2} \\
& H \equiv H(\boldsymbol{x}, \boldsymbol{p} ; t)=\dot{\boldsymbol{x}} \boldsymbol{p}-L(\dot{\boldsymbol{x}}, \boldsymbol{x} ; t)=\frac{1}{2} \boldsymbol{p}^{2}+\boldsymbol{p} \boldsymbol{K}(\boldsymbol{x} ; t) \\
& \boldsymbol{p}=\dot{\boldsymbol{x}}-\boldsymbol{K}(\boldsymbol{x} ; t) .
\end{aligned}
$$

The boundary conditions follow from the condition that the optimal path starts from the stable state as $t_{i} \rightarrow-\infty$, and therefore

$$
\begin{aligned}
& \boldsymbol{x}\left(t_{f}\right)=\boldsymbol{x}_{f} \\
& \boldsymbol{x}\left(t_{i}\right) \rightarrow x_{\mathrm{eq}}\left(t_{i}\right) \quad \boldsymbol{p}\left(t_{i}\right) \rightarrow 0 \quad S\left(t_{i}\right) \rightarrow 0 \quad \text { for } t_{i} \rightarrow-\infty .
\end{aligned}
$$

The function $S[x] / D$ evaluated along the optimal path determines the logarithm of the stationary probability distribution of the system $\rho(\boldsymbol{x})$ (for periodically-driven systems, the stationary distribution depends periodically on time),

$$
\rho(\boldsymbol{x})=\text { constant } \times \exp \left(-S\left[\boldsymbol{x}_{\mathrm{opt}}(t \mid \boldsymbol{x}, t)\right]\right) .
$$

Therefore, $\min S[x]$ is similar to the thermodynamic potential, and following Graham's suggestion (Haken 1975, Graham 1973,1989) it is sometimes called a non-equilibrium potential.

In fact, equations (26) and (27) describe extreme fluctuational paths. Optimal paths provide the global minimum to the action $S[x]$. It is clear from (20) and (28) that, for small $D$, these are optimal paths that possess physical significance, and not just extreme paths (26). 
3.2.1. System in equilibrium with thermal bath. The equations of motion (26) can easily be solved if the system is in equilibrium with a thermal bath, in which case the force $\boldsymbol{K}(\boldsymbol{x}, t)$ is independent of time and is a gradient force, i.e. $K(\boldsymbol{x}, t)=-\nabla U(\boldsymbol{x})$, so that

$$
\dot{\boldsymbol{x}}_{\mathrm{opt}}=\nabla U\left(\boldsymbol{x}_{\mathrm{opt}}\right) \quad \boldsymbol{p}_{\mathrm{opt}}=2 \nabla U\left(\boldsymbol{x}_{\mathrm{opt}}\right) \quad S(\boldsymbol{x})=2 U(\boldsymbol{x}) .
$$

It can be seen that the optimal fluctuational trajectory in this case is a mirror image (with respect to time) of the deterministic relaxational trajectory $\dot{x}=-\nabla U(x)$. This is a consequence of the principle of the symmetry of fluctuations with respect to time inversion for classical systems in thermal equilibrium in the absence of magnetic fields and rotation (Landau and Lifshitz 1980); see also section 3.2.3.

The existence of such optimal paths has been tested experimentally (Dykman et al 1992c) by investigation of the fluctuations of an electronic circuit modelling the system of interest, for which the system (21) corresponded to one-dimensional motion in the quartic bistable potential

$$
U(x)=-\frac{1}{2} x^{2}+\frac{1}{4} x^{4}
$$

driven by external white noise. Because no additional forces were applied, the system could be considered to be in thermal equilibrium at a temperature determined by the noise intensity and the damping constant, which are linked by the fluctuation dissipation theorem (Landau and Lifshitz 1980). The corresponding Langevin equation

$$
\dot{x}=x-x^{3}+f(t) \quad\left\langle f\left(t_{1}\right) f\left(t_{2}\right)\right\rangle=D \delta\left(t_{1}-t_{2}\right)
$$

was modelled with the circuit shown in figure 5(a). It is similar to that for the underdamped Duffing oscillator (figure 2), except that it contains only one integrator and that there is (for now) no periodic driving force. The equations for the points $\mathrm{A}$ and $\mathrm{B}$ are respectively

$$
\begin{aligned}
& C \frac{\mathrm{d} V_{2}}{\mathrm{~d} t^{\prime}}+\frac{1}{R_{1}} f^{\prime}\left(t^{\prime}\right)+\frac{1}{R_{2}} V_{1}=0 \\
& \frac{1}{10 R} V_{1}+\frac{1}{10 R} V_{2}+\frac{1}{R} V_{3}=0 .
\end{aligned}
$$

Noting (see figure 5(a)) that

$$
V_{3}=-\frac{V_{2}^{3}}{10}
$$

it is straightforward to show that

$$
\frac{\mathrm{d} V_{2}}{\mathrm{~d} t^{\prime}}+\frac{f^{\prime}\left(t^{\prime}\right)}{R_{1} C}+\frac{V_{2}^{3}}{R_{2} C}-\frac{V_{2}}{R_{2} C}=0 .
$$

By making the transformations $V_{2} \rightarrow x, t^{\prime} \rightarrow \tau t, f^{\prime}\left(t^{\prime}\right) \rightarrow f(t)$ as before, and choosing the circuit component values

$$
R=10 \mathrm{k} \Omega \quad R_{1}=R_{2}=100 \mathrm{k} \Omega \quad C=10 \mathrm{nF} \quad \tau=C R_{1}
$$

we then arrive at equation (31).

Starting with the system in the close vicinity of one of the stable states $\left(x_{i} \approx \pm 1\right)$, successive blocks of $x(t)$ time series were digitized with a Nicolet LAB80 data processor (a member of the NIC-80 series), and examined. The moment at which $x(t)$ eventually reached a pre-set value $x_{f}$ was noted, and the path by which it had reached that point was recorded. The process was then repeated, so as to build up an ensemble average of the paths leading to $x_{f}$. A typical example of the resultant prehistory probability density $p_{h}\left(x, t ; x_{f}, 0\right)$ is shown in figure 5(b). These results demonstrated (Dykman et al 1992c) for 


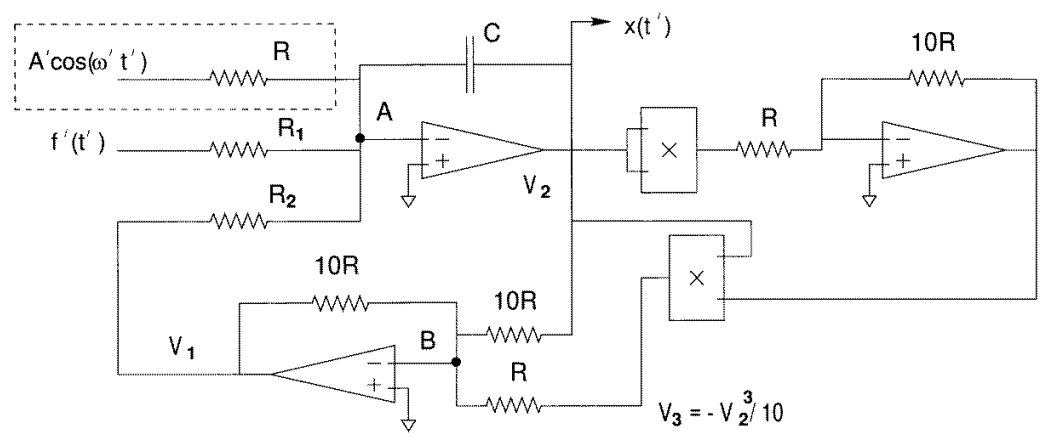

(a)

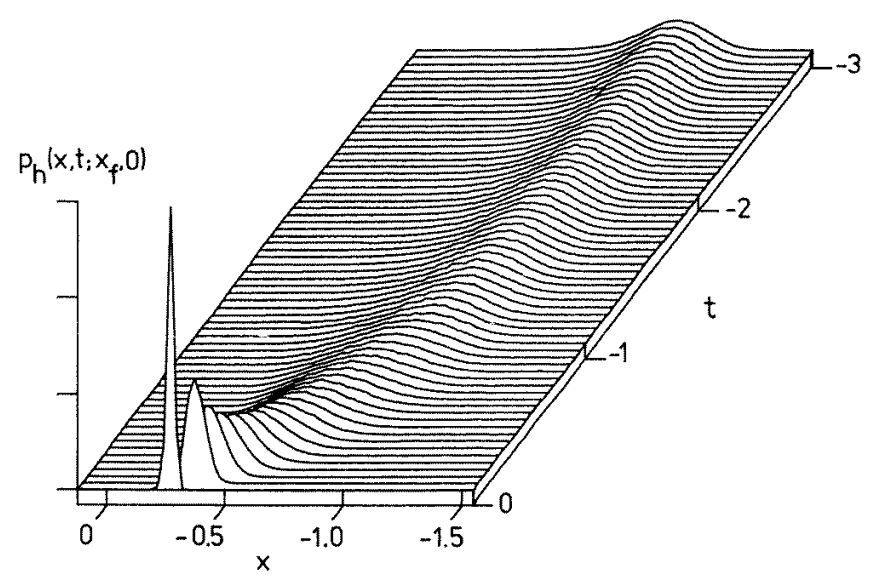

(b)

Figure 5. (a) A block diagram of an analogue electronic circuit modelling an overdamped double-well Duffing oscillator (21) and (32) (Dykman et al 1992c) either with, or without, the periodic force $A \cos \left(\omega^{\prime} t^{\prime}\right)$; (b) the prehistory probability density $p_{h}\left(x, t ; x_{f}, 0\right)$ for (21) and (32) (Dykman et al 1992c) measured for $A=0$ in the analogue electronic experiment for a final position $x_{f}=-0.30$ with $D=0.0701$.

the first time that optimal fluctuational paths are physically observable, and that using the new theoretical and experimental technique based on the prehistory probability distribution, it should be possible, in principle, to check many fundamental tenets of the theory of large fluctuations. Note that, although this experimental investigation, like all of the Lancaster nonlinear research up to 1996, was carried out with a Nicolet data processor, the more recent studies of large fluctuations described below depend on the PC-based Microstar ADC system described in section 2.3 .

3.2.2. System without detailed balance. For a non-potential or time-dependent force $\boldsymbol{K}(\boldsymbol{x} ; t)$ the Hamiltonian equations (26) with boundary conditions (27) become nonintegrable, generally speaking. The solutions of these equations can only be obtained in special cases, like in the case where the initial fluctuating system is underdamped (Dykman and Krivoglaz 1979, Ben-Jacob et al 1982) or where the drift coefficients $\boldsymbol{K}$ have a special symmetry (Ventcel' and Freidlin 1970, Graham and Tél 1986). In general, when solving the 
Hamiltonian equations one should be prepared to obtain singularities known in geometrical optics (Berry 1976), and also chaos. However, as we have mentioned before and will also outline later, the observable singularities in the pattern of optimal paths turn out to be different (Dykman et al 1994e).

It was first appreciated by Graham and Tél $(1984 a, b)$ that, since the simplest nonintegrable Hamiltonian system is the one with one degree of freedom and with the Hamiltonian that periodically depends on time (Guckenheimer and Holmes 1983), the simplest fluctuating system which may display singularities in the stationary probability distribution is an overdamped periodically-driven system with one degree of freedom. Respectively, the pattern of optimal paths of such a system should display singularities as well (Dykman et al 1996c, 1997a).

The simplest appropriate model is that of an overdamped fluctuating system driven by an additive sinusoidal force,

$$
\begin{aligned}
& \dot{x}=K(x ; t)+f(t) \quad K(x ; t)=-U^{\prime}(x)+A \cos \Omega t \\
& U(x)=-\frac{1}{2} x^{2}+\frac{1}{4} x^{4} .
\end{aligned}
$$

The model (32) has attracted a lot of attention recently in the context of stochastic resonance (see section 4). We consider a situation that is both non-adiabatic and nonlinear: neither $\Omega$ nor $A$ need be small; only the noise intensity $D$ will be assumed small.

Since the Hamiltonian $H(x, p ; t)$ is periodic in $t$, the set of paths $\{x(t), p(t)\}$ is also periodic: the paths that arrive at a point $\left(x_{f}, t_{f}\right)$ are the same as the paths that arrive at the point $\left(x_{f}, t_{f}+2 \pi / \Omega\right)$, but shifted in time by the period $2 \pi / \Omega$. Note, however, that any given individual optimal path is not itself periodic in time.

To acquire topological insight into the pattern of singularities of optimal paths (Dykman et al 1994e, Dykman and Smelyanskiy 1998b) we note that trajectories emanating from a stationary state lie on a Lagrangian manifold (LM) (Arnol'd 1978) in phase space $(x, t, p=\partial S / \partial x)$ (the unstable manifold of the corresponding state) and form a oneparameter set. The action $S(x, t)$ is a smooth single-valued function of position on the LM. It is a Lyapunov function: it is non-decreasing along the trajectories of the initial system in the absence of noise $\dot{x}=K(x ; t)$. The projections of trajectories in phase space onto configuration space form the extreme paths. Optimal paths are the extreme paths that give the minimal action to reach a given point $(x, t)$ in the configuration space. These are the optimal paths that can be visualized in an experiment via measurements of the prehistory probability distribution.

A periodically-driven system, being a system with 1.5 degrees of freedom (the coordinate and time), provides a good opportunity to look into (at least some of) the generic singularities of the optimal paths. The pattern of extreme paths, the LM and action surfaces calculated (Dykman et al 1997c) for the overdamped periodically-driven oscillator (32) are shown in figure 6 . It can be seen from figure 6 that, although there is only one path to a point $(x, t, p)$ in phase space, several different extreme paths may come from the stationary periodic state to the corresponding point $(x, t)$ in configuration space. These paths cross each other. This is a consequence of folding of the Lagrangian manifold.

A generic feature related to the folding of LMs is the occurrence of caustics in the pattern of extreme paths. Caustics are projections of the folds of an LM. They start at cusp points. Clearly, the area of the configuration space behind a caustic may not be reached along the paths that encounter it. If these paths formed the probability distribution in the corresponding range of dynamical variables, this distribution would be singular: the situation here is different from that with caustics in the WKB, as, in contrast to the wavefunction which is decaying on one side of the caustic and oscillating on the other side, the probability 


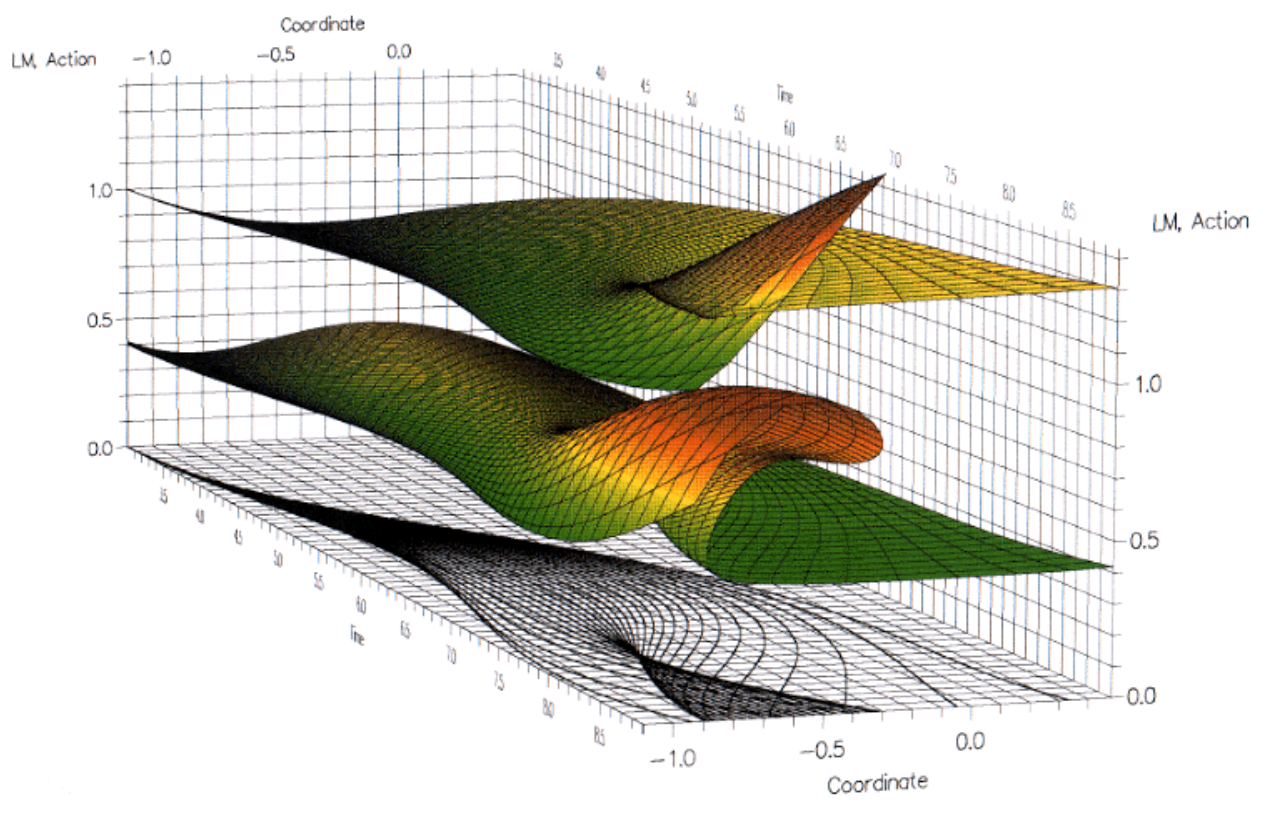

Figure 6. From top to bottom: action surface, Lagrangian manifold (LM) and extreme paths calculated for the system (32) using equations (26) with the initial conditions discussed by Dykman et al $(1997 \mathrm{~b}, \mathrm{c})$. They correspond to the analogue electronic circuit in figure 5(a), but with a periodic external force added as shown in that figure in the dashed box. Parameters for the system were: $A=0.264, \omega=1.2$. To clarify interrelations between singularities in the optimal paths pattern, action surface and LM surface, they are shown in a single figure: the action surface has been shifted up by one unit, and the LM scaled by a factor $1 / 2$ and shifted up by 0.4 .

distribution may not oscillate. Therefore, encountering a caustic is inappropriate, on a physical basis, and one would expect that the caustic should be 'hidden'.

The avoidance of caustics was analysed by Dykman et al (1994e) and can be understood from figure 6. It can be seen in figure 6 that the folds of the LM merge at the cusp point. The structure of an LM with two folds merging at the cusp gives rise to a swallowtail-type singularity in the action surface. The spinode edges of the action surface correspond to the caustics. The lower sheets of the action surface intersect along a line the projection of which on the coordinate place can be called a switching line. The switching line separates regions which are reached along different optimal paths, namely, the paths which correspond to the smaller value of the action. The switching line starts from the cusp point. The optimal paths terminate on the switching line prior to a caustic being encountered. This topological picture is generic (Dykman et al 1994e, Smelyanskiy et al 1997a), it explains both how caustics are avoided and what are the observable singularities of the pattern of optimal paths. The first numerical results on the formation of the singularities, avoidance of caustics and formation of switching lines in systems lacking detailed balance were obtained by Day (1987) and Jauslin (1987b), and the occurrence and avoidance of a turning point for a coloured-noise-driven system was obtained analytically by Dykman (1990). The generic topological features of the pattern of optimal paths had not been observed in experiments until the introduction of the analogue electronic approach that we describe later (see also section 6.4). 
In addition to the fact that large fluctuations occur only occasionally, their experimental investigation in non-equilibrium systems is also complicated by the fact that the coordinate space, in general, has two or more dimensions. This makes periodically-driven systems particularly advantageous for the analysis of the prehistory problem as the 'extra' coordinate is time, which is one of the variables of the prehistory probability distribution anyway.

The problem of statistics can be overcome, at least in part, by using a multichannel technique. Several dynamical variables of the system and the external force are recorded simultaneously, and then the statistics of all actual trajectories along which the system moves in a particular subspace of the coordinate space are analysed. Information about stochastic processes obtained in this way is much more detailed than that obtained by the standard method of measuring stationary probability distributions. In our technique, not only do we count rare events (i.e. arrivals of the system at a given point in configuration space), but we also learn how each of these events comes about.

The technique has been applied to the analysis of fluctuational dynamics in the analogue electronic circuit model of (32) shown in figure 5(a), with $A^{\prime} \neq 0$. An experimentally measured prehistory probability distribution for arrival at a particular point in configuration space is shown in figure 7. It is clear that the distribution is sharp and has a well defined ridge. From the top-plane plot and from the direct comparisons in figure 8 , it is evident that such ridges follow very closely the theoretical trajectories obtained by solving numerically the equations of motion for the optimal paths (26).

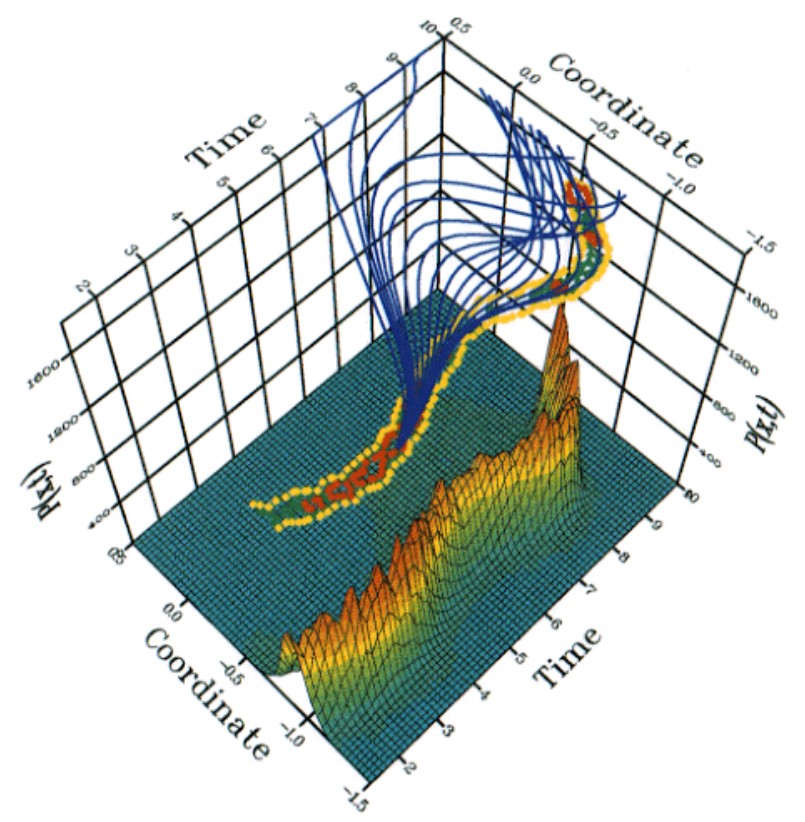

Figure 7. Distributions of fluctuational paths for the system (32) for a termination point inside the rectangle $-0.7 \leqslant x_{f} \leqslant-0.65,9.0 \leqslant t_{f} \leqslant 9.2$, with $A=0.264$ and $\omega=1.2$. The maxima of the distributions represent the optimal paths. The theoretical extreme paths and a contour plot of the distribution are shown on the top plane (Dykman et al 1997c). 
3.2.3. Width of the tube of fluctuational paths. Further insight into the physical meaning of the prehistory probability distribution can be gained from an analysis of the width of this distribution. The distribution broadening is due to the fact that, although the system is most likely to move to a given state along the optimal path, the actual fluctuational trajectories deviate from the optimal path. Therefore, the width of the tube of the paths for arrival to a given state is finite. This width is a fundamental characteristic of the fluctuation dynamics, and it is this width that is given by the width of the prehistory distribution.

Some calculated and measured widths are shown in the inset of figure 8 (Dykman et al 1996c). The theoretical technique developed in the cited paper is based on the analysis of the general expression for the prehistory probability distribution (20). To evaluate the path integral in this expression for the periodically-driven system (32) one expands the coordinate $x(t)$ into the orthonormal functions $\psi_{n}(t)$ which diagonalize the second variation of the action,

$$
x(t)=x_{\mathrm{opt}}\left(t \mid x_{f}, t_{f}\right)+\sum_{n} a_{n} \psi_{n}(t)
$$

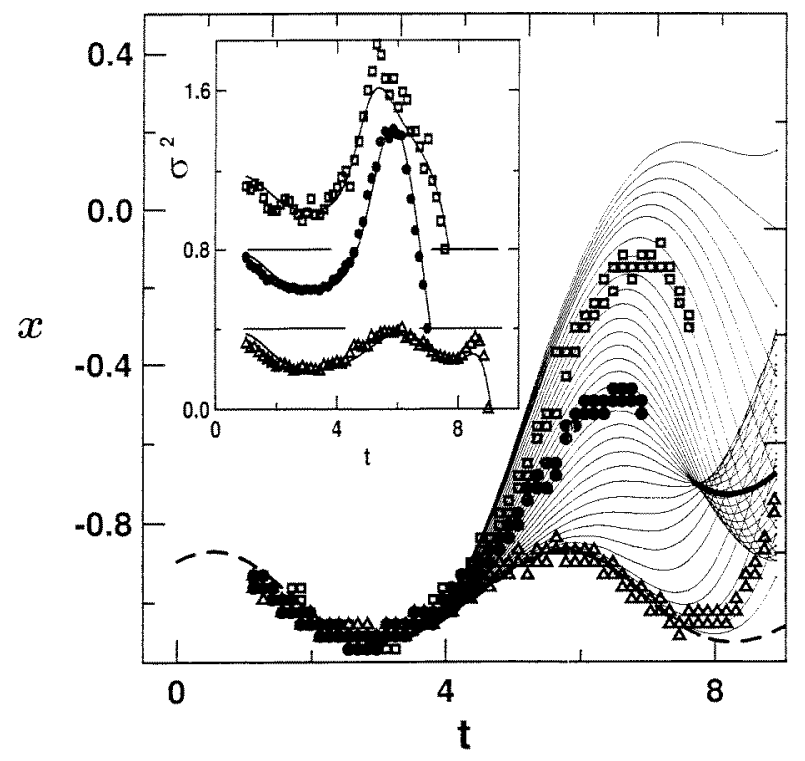

Figure 8. Comparison between measured optimal paths (data points) of the system (32) for three different termination points $\left(x_{f}, t_{f}\right)$ and the theoretically predicted pattern of extreme paths (fine lines). The periodically modulated stable state from whose vicinity the paths start is shown by the dashed curve; the calculated switching line is indicated by the heavy full curve starting from the cusp point. Inset: the reduced variances of the corresponding Gaussian distributions $\left(D \sigma^{2}\right)$ (displaced along the ordinate axis for clarity) are compared with the theory (36) and (37) (Dykman et al 1996c).

It follows from (23) that the functions $\psi_{n}(t)$ satisfy a Schrödinger-type equation

$$
-\ddot{\psi}_{n}+V(t)_{n}=\lambda_{n} \quad n \quad V(t)=\left[\frac{\partial^{2} K}{\partial x \partial t}+\frac{1}{2} \frac{\partial^{2} K^{2}}{\partial x^{2}}\right]_{\mathrm{opt}}
$$

with the boundary conditions $\psi_{n}\left(t_{i}\right)=\psi_{n}\left(t_{f}\right)=0$ (in (34) the derivatives of $K \equiv K(x ; t)$ are evaluated for $\left.x=x_{\mathrm{opt}}\left(t \mid x_{f}, t_{f}\right)\right)$. 
For trajectories $x(t)$ close to the optimal path, the $a_{n}$ in (33) are small, and the action $S[x(t)]$ is quadratic in $a_{n}$ (unless $\left(x_{f}, t_{f}\right)$ is close to the cusp: see later),

$$
\begin{aligned}
& S[x(t)]=S\left(x_{f}, t_{f}\right)+s\left(\left\{a_{n}\right\}\right) \quad s\left(\left\{a_{n}\right\}\right)=\frac{1}{2} \sum_{n} \lambda_{n} a_{n}^{2} \\
& S\left(x_{f}, t_{f}\right) \equiv S\left[x_{\mathrm{opt}}\left(t \mid x_{f}, t_{f}\right)\right] .
\end{aligned}
$$

If one writes the path integral (20) as an integral over all $a_{n}$ and substitutes equations (33) and (35) into (20), one obtains

$$
\begin{aligned}
& p_{h}\left(x, t \mid x_{f}, t_{f}\right)=M \exp \left(-\frac{\left[x-x_{\mathrm{opt}}\left(t \mid x_{f}, t_{f}\right)\right]^{2}}{2 D \sigma^{2}\left(t \mid x_{f}, t_{f}\right)}\right) \\
& \sigma^{2}\left(t \mid x_{f}, t_{f}\right)=\sum_{n} \lambda_{n}^{-1} \quad{ }_{n}^{2}(t), M=\left(2 \pi D \sigma^{2}\right)^{-1 / 2} .
\end{aligned}
$$

It can be seen from (36) that, near the maximum, the distribution $p_{h}$ is Gaussian in the distance of the point $(x, t)$ from the optimal path $x_{\mathrm{opt}}\left(t \mid x_{f}, t_{f}\right)$ (cf Dykman et al 1992c).

It follows from equation (36) that by investigating $p_{h}$ not only can one find directly the optimal path itself, but one can also analyse the shape of the tube of paths arriving at a given point $\left(x_{f}, t_{f}\right)$. Away from the cusp point, the width of this tube is proportional to $D^{1 / 2}$, which justifies the expansion of $S$ in the coefficients $a_{n}$ (35).

The reduced width of the distribution (36) $\sigma\left(t \mid x_{f}, t_{f}\right)$ is given by the Green function of (34) at zero energy. For an arbitrary periodic force $K$ in (32) its evaluation reduces (Dykman et al 1996c) to the solution of an ordinary differential equation

$$
\begin{aligned}
& \sigma^{2}\left(t \mid x_{f}, t_{f}\right)=\int_{t}^{t_{f}} \mathrm{~d} t_{1} \exp \left[-2 \int_{t}^{t_{1}} \mathrm{~d} t_{2} \beta\left(t_{2}\right)\right] \\
& \dot{\beta}+\beta^{2}=V(t) \quad \beta\left(t_{i}\right)=\beta\left(t_{i}+T\right) \quad \text { for } t_{i} \rightarrow-\infty .
\end{aligned}
$$

Clearly, $\sigma\left(t \mid x_{f}, t_{f}\right)$ is independent of $x_{f}, t_{f}$ for $t_{f}-t \gg \tau_{r}$ : it gives the reduced width of the stationary Gaussian distribution about $x^{(0)}(t)$. As shown in the inset of figure 8, equations (25) and (37) provide an excellent description of the experimental data.

3.2.4. Critical behaviour of the paths distribution. The only generic structurally stable singularities of the pattern of extreme paths of $S[x]$ (23) in two-dimensional (2D) systems are caustics and cusps (Arnol'd 1992). Since caustics may not be observed in the pattern of optimal paths, it is particularly interesting to investigate the distribution $p_{h}$ near cusp points. At the cusp $\left(x_{\mathrm{c}}, t_{\mathrm{c}}\right)$ one of the eigenvalues $\lambda_{n}$ becomes equal to zero (Berry 1976, Schulman 1996) (we assume that this happens to the eigenvalue $\lambda_{0}$ ). Equation (36) does not apply if the final point $\left(x_{f}, t_{f}\right)$ is close to $\left(x_{\mathrm{c}}, t_{\mathrm{c}}\right)$; in particular, $\sigma$ diverges for $\lambda_{0}=0$.

In a certain sense a cusp point is similar to the critical point of the first-order phase transition. On one side of the cusp point there exists an area which can be reached along the paths that belong to one or other of two coexisting types. This is similar to the occurrence of two coexisting phases on one side of a critical point. The switching line is an analogue of the phase transition line. As in the case of the critical point, the motion of the system is slowed down near the cusp point, and there arises a 'soft mode' $\psi_{\mathrm{c} 0}(t)$. Therefore, the width of the prehistory probability distribution should critically increase.

In contrast to a true critical point, at the cusp point the spectrum $\lambda_{n}$ is discrete, and therefore one would expect that Landau-type theory should apply to the prehistory distribution. We now present results for the case where the final point $\left(x_{f}, t_{f}\right)$ is precisely the cusp (Dykman et al 1996c). 
It is known from optics and quantum mechanics (Berry 1976, Schulman 1996) that at the cusp point it is necessary to keep, in the expansion of the action $S$ (35), the higher-order terms in the amplitude $a_{0}$ of the 'soft mode' $\psi_{0}(t)$,

$$
\begin{gathered}
S[x(t)]=S\left(x_{\mathrm{c}}, t_{\mathrm{c}}\right)+s_{\mathrm{c}}\left(\left\{a_{n}\right\}\right) \quad s_{\mathrm{c}}\left(\left\{a_{n}\right\}\right) \approx \frac{1}{4} g_{0} a_{0}^{4}+\frac{1}{2} \sum_{n>0} \lambda_{n} a_{n}^{2}+\frac{1}{2} a_{0}^{2} \sum_{n>0} g_{n} a_{n} \\
{ }_{0}(t) \equiv \psi_{\mathrm{c} 0}(t) \propto \exp \left[\int^{t} \mathrm{~d} t^{\prime} \beta\left(t^{\prime}\right)\right] .
\end{gathered}
$$

Absence of the term $a_{0}^{3}$ in the expansion (38) is the signature of the fact that we are dealing with the cusp point. We note that all eigenvalues $\lambda_{n}$ are positive if the final state $\left(x_{f}, t_{f}\right)$ lies near the stable state, and they change sign along extreme paths only provided such a path encounters a caustic, which does not happen for physically meaningful extreme paths, that is for optimal fluctuational paths. A cusp point is the only point on the optimal path where the eigenvalue $\lambda_{0}$ becomes equal to zero.

If we change to integration over the coefficients $a_{n}$ in the path integral (20), and integrate over all $a_{n>0}$ with account taken of equations (35) and (38) we obtain the prehistory probability distribution for the paths coming to the cusp point in the form

$$
\begin{aligned}
p_{h}\left(x, t ; x_{\mathrm{c}}, t_{\mathrm{c}}\right) & =M_{\mathrm{c}} \int_{-\infty}^{\infty} \mathrm{d} a_{0} \exp \left[-\mathcal{F}\left(a_{0} \mid x_{\mathrm{c}}, t_{\mathrm{c}}\right) / D\right] \\
& \times \exp \left[-\frac{\left[x-x_{\mathrm{opt}}\left(t \mid x_{\mathrm{c}}, t_{\mathrm{c}}\right)-a_{0} \psi_{\mathrm{c} 0}(t)\right]^{2}}{2 D \sigma_{\mathrm{c}}^{2}\left(t \mid x_{\mathrm{c}}, t_{\mathrm{c}}\right)}\right] \\
\mathcal{F}\left(a_{0} \mid x_{\mathrm{c}}, t_{\mathrm{c}}\right) & =\frac{1}{4} g a_{0}^{4} \quad g=g_{0}-\frac{1}{2} \sum_{n>0} \lambda_{n}^{-1} g_{n}^{2} .
\end{aligned}
$$

The function $\sigma_{\mathrm{c}}^{2}$ in (39) is given by equation (36) with the term $n=0$ being eliminated from the sum; $M_{\mathrm{c}} \equiv M_{\mathrm{c}}(t)$ is a normalization constant.

Very close to the cusp point, i.e. for very small $\left(t_{\mathrm{c}}-t\right)$ the shape of the distribution (39) is dominated by diffusion, so that the distribution is Gaussian; the term $a_{0} \psi_{\mathrm{c} 0}$ can be neglected, and $\sigma_{\mathrm{c}}(t) \approx\left(t_{\mathrm{c}}-t\right)^{1 / 2}$.

For larger $\left(t_{\mathrm{c}}-t\right)$ we have from (39)

$$
\begin{aligned}
& p_{h}\left(x, t ; x_{\mathrm{c}}, t_{\mathrm{c}}\right)=\tilde{M}_{\mathrm{c}} \exp \left\{-\frac{g}{4 D}\left[\frac{x-x_{\mathrm{opt}}\left(t \mid x_{\mathrm{c}}, t_{\mathrm{c}}\right)}{\psi_{\mathrm{c} 0}(t)}\right]^{4}\right\} \\
& \tau_{\mathrm{r}} \gtrsim t_{\mathrm{c}}-t \gg D^{1 / 2} g^{1 / 2} / \dot{\psi}_{\mathrm{c} 0}^{2}\left(t_{\mathrm{c}}\right) .
\end{aligned}
$$

Equation (40) shows that, at the cusp point, fluctuations about the optimal path become strongly non-Gaussian, and there occurs critical broadening of the paths distribution. In the range (40) the shape of the distribution of fluctuational paths to the cusp point is described by the Landau-type expression for the distribution of fluctuations of the order parameter near the critical point, with the width being dependent on the distance $t-t_{\mathrm{c}}$ to the cusp point along the optimal path. The prehistory probability distribution can be used to reveal this new critical effect.

The characteristic width of the distribution (40) is $\sim D^{1 / 4}$, and its broadening is determined by the soft mode $\psi_{\mathrm{c} 0}(t)$. This mode is localized within the range $t_{\mathrm{c}}-t \lesssim \tau_{\mathrm{r}}$. For $t_{\mathrm{c}}-t \gg \tau_{\mathrm{r}}$ the distribution (39) goes over into the stationary Gaussian distribution about the attractor $x_{\mathrm{eq}}(t)$. The measured and calculated evolutions of the distribution with $\left(t_{\mathrm{c}}-t\right)$ are in good agreement: see figure 9. The critical behaviour of the distribution of paths coming to a cusp point is much more pronounced than that of the statistical distribution near a cusp, 


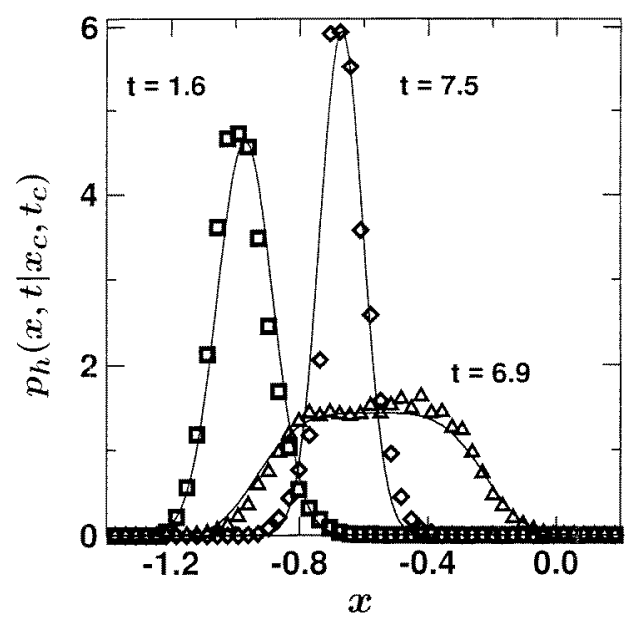

Figure 9. Cross sections of the prehistory probability distribution for fluctuations to the cusp point in figure $8\left(x_{\mathrm{c}} \approx-0.70, t_{\mathrm{c}} \approx 7.69\right)$ for three values of $t$. The distribution is Gaussian very close to, and far from, the cusp; but is critically broadened and strongly non-Gaussian, cf equation (40), at intermediate values of $t$ (Dykman et al 1996c).

where it is seen primarily in a blowing up of the prefactor (Dykman et al 1994e, Maier and Stein 1996a).

\subsection{Time asymmetry of classical fluctuations}

The results discussed briefly above demonstrate clearly that patterns of optimal paths, and some of the singularities in these patterns, are experimentally observable, and that it is much easier to observe these singularities than the features of the tails of a stationary probability distribution. The role of these tails and of large fluctuations in general is particularly important (Landauer 1975, 1978, 1988) for systems away from thermal equilibrium, as there is no general principle that determines the fluctuation probabilities in such systems.

It is legitimate, therefore, to pose a question about general properties of fluctuation dynamics and patterns of optimal paths in non-equilibrium systems. One of the most important properties that we address here is the symmetry, with respect to time inversion, between optimal fluctuational paths and relaxational paths in the absence of fluctuations, and in what follows we limit the discussion to Markov systems. This symmetry is a feature of systems in thermal equilibrium. The very occurrence of a fluctuational path which is a timeinversed relaxational path is a consequence of microscopic reversibility of closed systems, and in that sense is trivial, but here we are talking about the optimal paths. The timereversal symmetry between optimal fluctuational and relaxational paths in linear Markov systems was first shown by Onsager and Machlup (1953).

The paths symmetry in thermal Markov systems is a consequence of detailed balance and applies to various types of Markov systems, including dynamical ones (Ventcel' and Freidlin 1970, Graham and Tél 1986, Dykman et al 1989, Maier and Stein 1993b) or systems described by master equations (Hunt et al 1990, Ross et al 1992, Dykman et al 1994c). The condition of detailed balance states that each individual transition in the system is balanced, i.e. the number of transitions $\boldsymbol{x} \rightarrow \boldsymbol{x}^{\prime}$ per unit time is equal to the number of transitions $\boldsymbol{x}^{\prime} \rightarrow \boldsymbol{x}$ (for states with continuous even variables $\boldsymbol{x}$ one should speak about the densities of transitions, i.e. the relative number of transitions per infinitesimal 'volume' 
centred at $\boldsymbol{x}$ ). This condition always holds true in systems in thermal equilibrium (Landau and Lifshitz 1980), as there are no currents in such systems. It requires that the probabilities of elementary transitions $w\left(\boldsymbol{x} \rightarrow \boldsymbol{x}^{\prime}\right)$ be balanced (cf Kac 1959, Graham 1973) so that the ratio of the probabilities of forward and backward transitions between two states is equal to the ratio of the probabilities of the transitions via an intermediate state. In other words, the ratio of the probabilities of transitions back and forth is independent of the path (cf Dykman et al 1994c).

$$
\frac{w\left(\boldsymbol{x} \rightarrow \boldsymbol{x}^{\prime}\right)}{w\left(\boldsymbol{x}^{\prime} \rightarrow \boldsymbol{x}\right)}=\frac{w\left(\boldsymbol{x} \rightarrow \boldsymbol{x}_{1}\right)}{w\left(\boldsymbol{x}_{1} \rightarrow \boldsymbol{x}\right)} \frac{w\left(\boldsymbol{x}_{1} \rightarrow \boldsymbol{x}^{\prime}\right)}{w\left(\boldsymbol{x}^{\prime} \rightarrow \boldsymbol{x}_{1}\right)} .
$$

In particular, as applied to systems described by the Langevin equation (21) in the case of a time-independent force $\boldsymbol{K}(\boldsymbol{x})$, equation (41) holds if the integral $\int \mathrm{d} \boldsymbol{x} \boldsymbol{K}(\boldsymbol{x})$ is path-independent, i.e. the force is gradient, $\boldsymbol{K}(\boldsymbol{x})=-\nabla U(\boldsymbol{x})$. The symmetry between optimal and relaxational paths in this case was discussed in section 3.2.1. We note that the condition (41) on its own does not guarantee that there is detailed balance in the system, as there still may be stationary solutions with current, as happens in the case of a Brownian particle in a tilted washboard potential (Graham and Tél 1986, Risken 1993).

In terms of the transition probability densities $\rho\left(\boldsymbol{x}_{f}, t_{f} \mid \boldsymbol{x}_{i}, t_{i}\right)$, the condition of detailed balance for the stationary distribution $\rho(\boldsymbol{x})$ for a closed system in the absence of magnetic field and rotation takes the form (Van Kampen 1990)

$$
\rho\left(\boldsymbol{x}_{2}, t \mid \boldsymbol{x}_{1}, 0\right) \rho\left(\boldsymbol{x}_{1}\right)=\rho\left(\boldsymbol{x}_{1}, t \mid \boldsymbol{x}_{2}, 0\right) \rho\left(\boldsymbol{x}_{2}\right) \quad(t>0) .
$$

Since for Markov systems the joint transition probability density factorizes into the probability densities of individual transitions,

$$
\rho\left(\boldsymbol{x}_{f}, t_{f} ; \boldsymbol{x}, t \mid \boldsymbol{x}_{i}, t_{i}\right)=\rho\left(\boldsymbol{x}_{f}, t_{f} \mid \boldsymbol{x}, t\right) \rho\left(\boldsymbol{x}, t \mid \boldsymbol{x}_{i}, t_{i}\right), \quad t_{f}>t>t_{i}
$$

it follows from the definition of the prehistory probability density (20) and (42) that

$$
\begin{gathered}
p_{h}\left(\boldsymbol{x}, t ; \boldsymbol{x}_{f}, t_{f}\right)=\frac{\rho\left(\boldsymbol{x}_{f}, t_{f} \mid \boldsymbol{x}, t\right) \rho\left(\boldsymbol{x}, t \mid \boldsymbol{x}_{i}, t_{i}\right)}{\rho\left(\boldsymbol{x}_{f}, t_{f} \mid \boldsymbol{x}_{i}, t_{i}\right)} \stackrel{t_{i} \rightarrow-\infty}{=} \frac{\rho\left(\boldsymbol{x}_{f}, t_{f} \mid \boldsymbol{x}, t\right) \rho(\boldsymbol{x})}{\rho\left(\boldsymbol{x}_{f}\right)} \\
=\rho\left(\boldsymbol{x},\left|t-t_{f}\right| \mid \boldsymbol{x}_{f}, 0\right) \quad t<t_{f}
\end{gathered}
$$

(Schulman 1991, Morillo et al 1997).

Equations (43) show that, for Markov processes obeying detailed balance, the time evolution of the prehistory probability distribution is identical (Morillo et al 1997) to the evolution of the corresponding (i.e. measured for the same remote state $\left.\left(x_{f}, t_{f}\right)\right)$ conditional probability of the reciprocal transition (the relation of the prehistory probability distribution to the 'conventional' transition probability in systems with detailed balance, as well as transient effects, were discussed by Vugmeister et al (1997)). Thus, one can investigate experimentally the presence (or lack) of detailed balance for a given stochastic process by measuring the time evolution of both distributions.

To understand in more detail how an experiment on temporal asymmetry can be performed let us consider a typical large fluctuation. As before (figure 4), a component $x$ of some dynamical variable $\boldsymbol{x}$ departs temporarily from the vicinity of the stable state, moves to a remote state $x_{f}$ and then returns. Extending our discussion in the previous section, however, we will now be interested not only in the outgoing (fluctuational) path away from the attractor, but also in the relaxational path back to the attractor again. The variable $x$ might represent the voltage(s) in an electrical circuit (e.g. in an analogue experiment), or the phase of the order parameter in a SQUID (superconducting quantum interference device) (Kautz 1996) or in an optically bistable device (Dykman et al 1995a), or the number densities of species in a chemical reaction (Dykman et al 1994f). 
An important feature of Markov systems is that, once the optimal outburst of the random force $f_{\text {opt }}(t)$ has brought the system to a given state $x_{f}$, the random force immediately (over its correlation time, which is equal to zero for white noise) dies down to its mean-square root value (Dykman 1990). Therefore, the system is then moving 'on its own', i.e. the section of the trajectory after $\left(\boldsymbol{x}_{f}, t_{f}\right)$ should correspond just to the relaxational path-the path the system would follow if it had been prepared in the state $\left(\boldsymbol{x}_{f}, t_{f}\right)$ and then let go, with noise being weak.

Optimal relaxational paths down to the stable state are the paths in the absence of noise. They are also described by the Hamiltonian equations (26), with the solution

$$
\dot{\boldsymbol{x}}=\boldsymbol{K}(\boldsymbol{x} ; t) \quad \boldsymbol{p}=0 .
$$

The set of trajectories (44) approaching the stable state of the system and the corresponding fixed point $\boldsymbol{x}=\boldsymbol{x}_{\mathrm{eq}}, \boldsymbol{p}=0$ in phase space, forms the stable invariant manifold of this fixed point. We note that, in contrast to the relaxational paths (44), optimal fluctuational paths lie on the unstable invariant manifold of the same fixed point, as we discussed above, and for these paths the momentum $\boldsymbol{p}$ is not equal to zero. However, although the decay of fluctuations was well understood (Van Kampen 1990), whether or how the growth of a fluctuation along the optimal path, which corresponds to the trajectory of the unstable manifold, might be observed experimentally remained uncertain for a long time.

The results discussed above demonstrate, however, the physical reality both of the optimal fluctuational paths and of the singular features in their pattern: see also Dykman et al (1992c, 1996a), Luchinsky (1997) and Luchinsky and McClintock (1997). Thus, a direct experimental investigation of time symmetry properties of fluctuational motion becomes possible with only a minor modification of the original experimental technique (see section 3.2.1). In the modified technique (Luchinsky 1997, Luchinsky and McClintock 1997) the state of the system is monitored continuously until eventually, as shown in figure 10(a), a large fluctuation reaches the voltage $\boldsymbol{x}_{f}$. But now the interesting region of the path includes not only the fluctuational part $f$ coming from the stable state to $\boldsymbol{x}_{f}$, but also the relaxational part $r$ leading back towards the stable state, and both parts of the path are stored. An ensemble average of the corresponding trajectories (figure 10(b)), built up over a period of time (typically weeks), creates the distribution $p_{f}(\boldsymbol{x}, t) \equiv \rho\left(\boldsymbol{x}_{f}, t_{f} ; \boldsymbol{x}, t \mid \boldsymbol{x}_{i}, t_{i}\right) / \rho\left(\boldsymbol{x}_{f}, t_{f} \mid \boldsymbol{x}_{i}, t_{i}\right)$ which applies for $t>t_{f}$ as well as $t<t_{f}$, and gives the probability density of the system being at $\boldsymbol{x}$ at time $t$ (with $t>t_{f}$ or $t<t_{f}$ ) if it was at $\boldsymbol{x}_{f}$ at time $t_{f}=0$ and $\boldsymbol{x}_{i} \approx \boldsymbol{x}_{\mathrm{eq}}$ at time $t_{i} \rightarrow-\infty$.

We now consider the application of these ideas to the fluctuations of three very different example systems that can be used to describe a wide range of physical phenomena (Dykman et al 1995d, Van Kampen 1990, Risken 1993, Haken 1975).

3.3.1. Symmetry of fluctuations in equilibrium Markov systems. Our first example is the overdamped Duffing oscillator (31) already discussed above (section 3.2.1), modelling a bistable system in thermal equilibrium near one of its stable states. Figure 10(a) shows a measured $p_{f}(x, t)$. It is evident that the relaxational and fluctuational parts of the distribution are symmetrical, which would only be expected under conditions of detailed balance. Indeed, one can rewrite equation (43) for the function $p_{f}(\boldsymbol{x}, t)$ for $t>0$ as

$p_{f}(\boldsymbol{x}, t)=\frac{\rho\left(\boldsymbol{x}, t \mid \boldsymbol{x}_{f}, 0\right) \rho\left(\boldsymbol{x}_{f}, 0 \mid \boldsymbol{x}_{i}, t_{i}\right)}{\rho\left(\boldsymbol{x}_{f}, 0 \mid \boldsymbol{x}_{i}, t_{i}\right)}=\rho\left(\boldsymbol{x}, t \mid \boldsymbol{x}_{f}, 0\right) \quad t>0$.

The aforementioned symmetry follows directly from comparison of equations (43) and (45). 


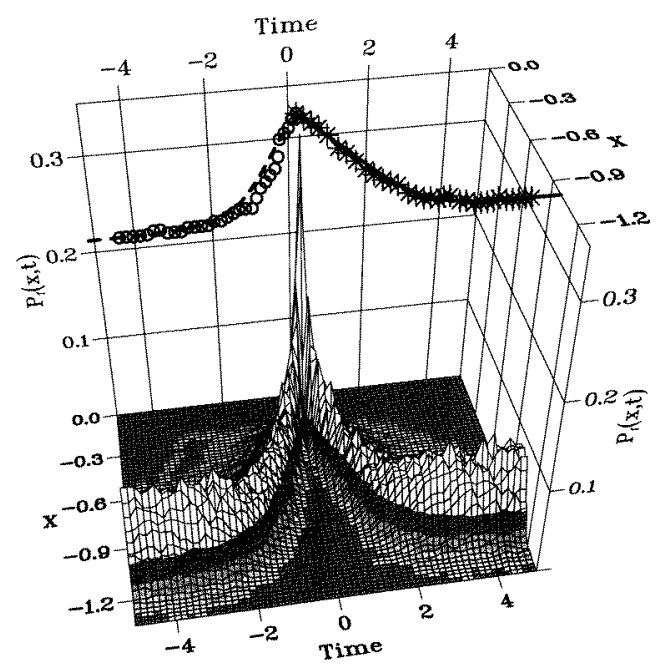

(a)

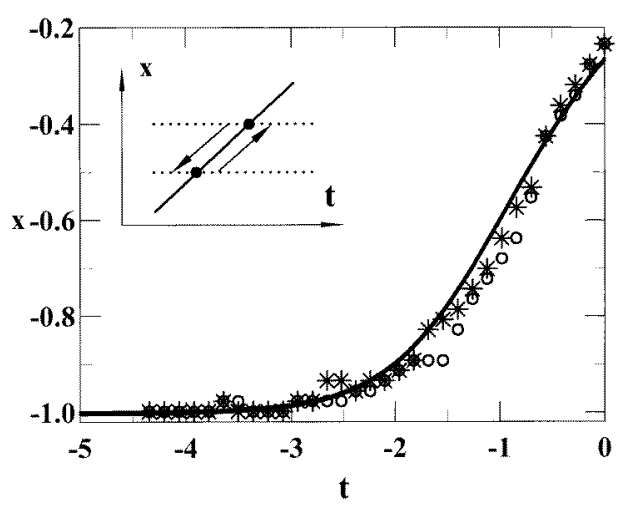

(b)

Figure 10. (a) The probability distribution $p_{f}(x, t)$ built up by ensemble averaging a sequence of trajectories like those in figure 4 for the thermal equilibrium system (31). The top plane plots the positions of the ridges of $p_{f}(x, t)$ for the fluctuational (open circles) and relaxational (asterisks) parts of the trajectory for comparison with theoretical predictions (curves) based on (26). (b) Direct comparison of the measured fluctuational (circles) and time-reversed relaxational (asterisks) ridges of the distribution in (a) with the theoretical prediction (full curve) from (26), demonstrating the time-reversal symmetry of the two kinds of path (Luchinsky 1997).

One also sees from figure 10(a) that the ridges of the distribution follow closely the deterministic trajectory found from (26), plotted as the full curve in figure 10(b). Hence, in the macroscopic limit, where the width of the distribution tends to zero (because $D \rightarrow 0$ ) and one observes only the positions of the ridges, the paths to/from $x_{f}$ themselves become symmetric in time.

An immediate question arising from the Hamiltonian theory relates to the physical significance of the quantity $\boldsymbol{p}$, which plays the role of a momentum in (26). This point is sometimes glossed over in the literature, with some authors describing $\boldsymbol{p}$ as a mere 'theoretical abstraction'. However, as is clear from the way we derived the Hamiltonian equations (26), the momentum $\boldsymbol{p}(t)$ on the optimal path is directly proportional to the 
optimal force $f_{\text {opt }}(t)$ that drives the system to a given remote state (Dykman and Krivoglaz 1979, 1984, Dykman 1990). Therefore, in our analogue experiment, $p$ can be identified as the averaged value of the force driving the fluctuation, which is of course accessible to experimental measurement (as is also the case for the random force in Monte Carlo simulations of stochastic processes). Thus, it becomes possible to perform direct tests of the prediction of an optimal force.

Luchinsky and McClintock (1997) have made simultaneous measurements of $x(t)$ and of the corresponding trajectories of the random force $f(t)$ in the analogue model of (31) during transitions between the potential wells, i.e. setting $x_{f}=0$ on the local potential maximum. Examples of actual trajectories (of the random force $f(t)$ ) during escapes from each of the potential wells are shown as a function of the coordinate in the inset of figure 11, where they are compared to the theoretical escape paths obtained from equations (26) in the phase space $(x, p)$ of the auxiliary Hamiltonian system. A distribution was built up by ensemble averaging a few hundred such trajectories, and the positions of the maxima of this distribution are shown in figure 11 for comparison with the theoretical escape trajectories. We emphasize that, in the limit of weak noise, such escape events are extremely rare. Although their statistics are consequently rather poor, the data clearly demonstrate: (i) that the averaged value of the force driving the fluctuation follows closely the deterministic trajectory corresponding to the optimal force; (ii) that $p$ can be related to this optimal force; (iii) that, as anticipated, $p \neq 0$ during the fluctuational part of the path and $p=0$ within experimental error during the relaxational part; and (iv) that the Hamiltonian theory (curves) describes very well both parts of the fluctuation.

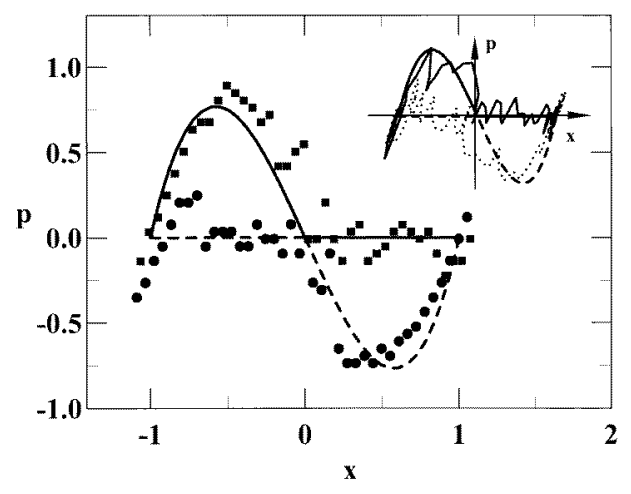

Figure 11. Demonstration of time-asymmetric features of fluctuations in the thermal equilibrium system (31). The inset shows $p(x)$ for two typical transitional paths from $x=-1$ to $x=1$ (full jagged line) and in the opposite direction (dotted jagged line). The main figure shows the paths traced out by the ridges of the $p_{f}(p, x)$ distribution created from an ensemble average of such transitions. The transitional path from $x=-1$ to $x=1$ is shown by squares, and the reverse transition by filled circles. The full and dashed curves are the corresponding paths predicted from (26) (Luchinsky and McClintock 1997).

3.3.2. Asymmetry of fluctuations in non-equilibrium systems. Our second example is the archetypal non-equilibrium system (32) of section 3.2.2. This system does not obey the detailed balance condition, and the ridges of the fluctuational and relaxational parts of the measured $p_{f}(x, t)$ are found to differ markedly in shape (Luchinsky and McClintock 1997). The paths that they trace out (data points), compared in figure 12(a) with theoretical 


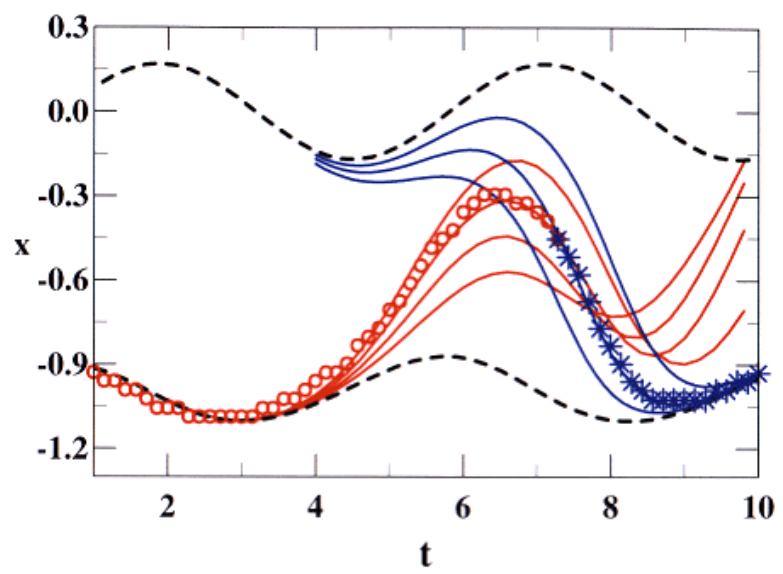

(a)

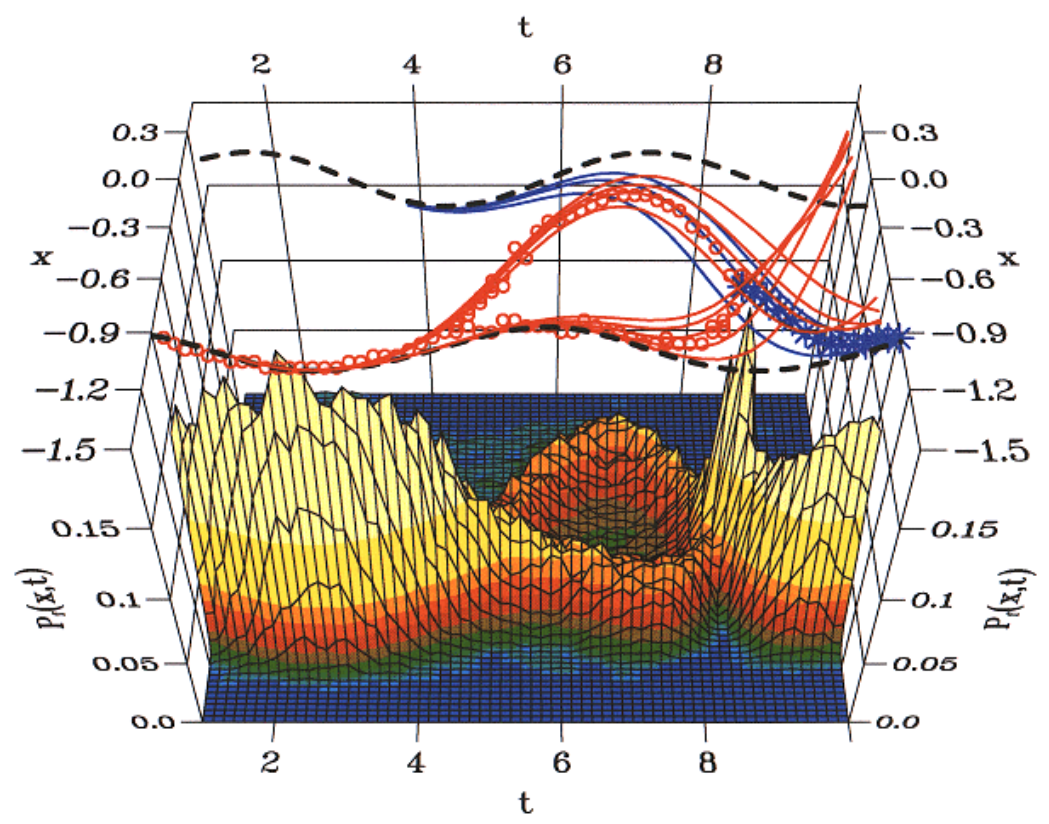

(b)

Figure 12. Fluctuational behaviour measured and calculated for an electronic model of the nonequilibrium system (32) with $A=0.264, D=0.012$. (a) Fluctuational and relaxational paths (red circles and blue asterisks, respectively) to/from the remote state $x_{f}=-0.46, t=0.73$, found by tracing the ridges of a measured $p_{f}(x, t)$ distribution. The time-dependent stable and unstable states near $x=-1$ and $x=0$ are shown by dashed curves. The fluctuational and relaxational paths calculated from (26) are shown as red and blue curves, respectively. (b) The measured $p_{f}(x, t)$ for a remote state $x_{f}=-0.63, t=0.83$ that lies on the switching line. (c) Fluctuational (red circles) and relaxational (blue asterisks) paths determined by tracing the ridges of the distribution in (b), and compared with the corresponding (red and blue) theoretical curves predicted from (26) (Luchinsky and McClintock 1997).

predictions (curves) calculated from (26), clearly demonstrate that the most probable fluctuational trajectory to $\left(x_{f}, t_{f}\right)$ does not correspond to what one would obtain by time 


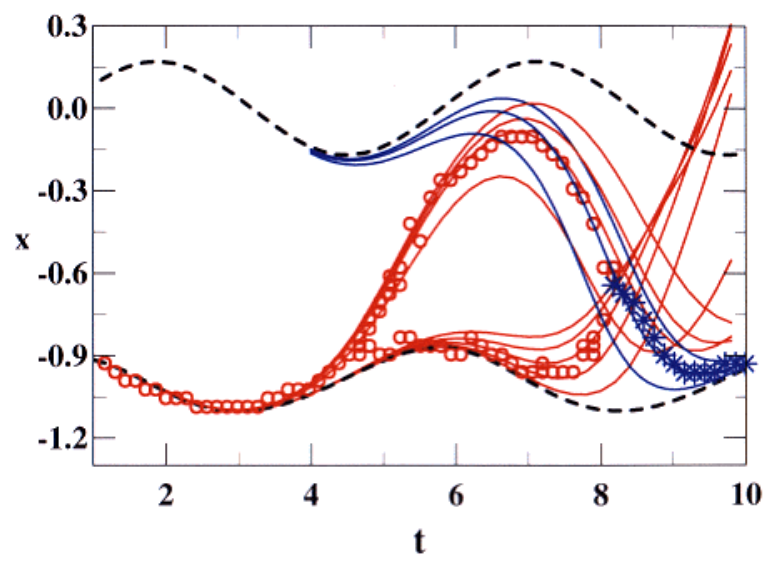

(c)

Figure 12. Continued.

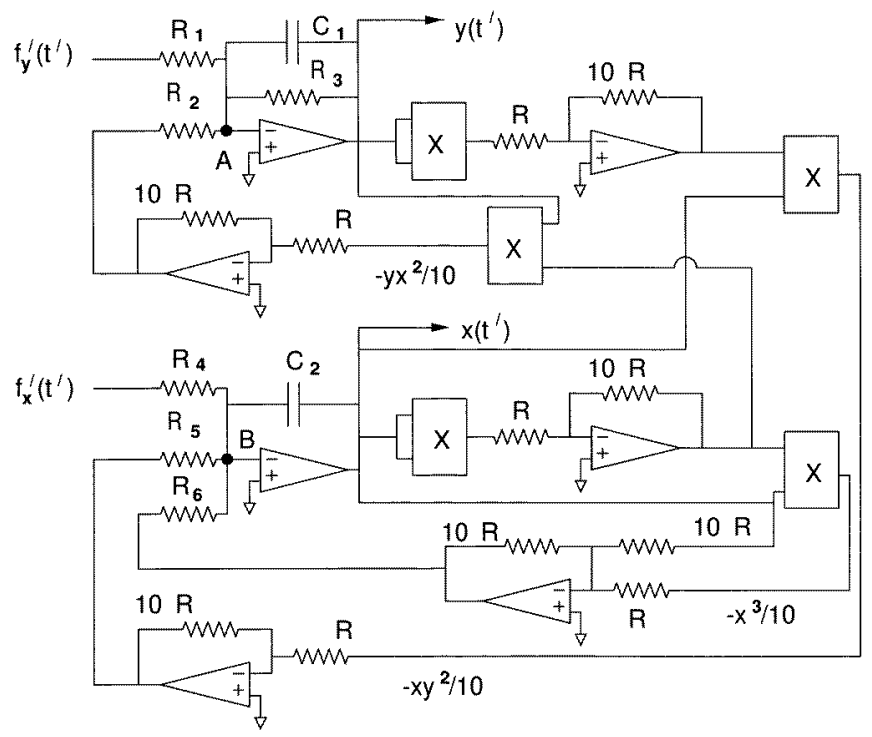

Figure 13. A block diagram of the analogue electronic circuit modelling the Maier and Stein system (46).

reversing the relaxational trajectory. Figure 12(b) shows the measured $p_{f}(x, t)$ when $x_{f}$ is placed on the calculated switching line (Dykman et al 1994e; and see section 3.2.2), a singularity separating regions that are approached via different fluctuational paths. The relaxational tail leading back to the stable state $x_{\mathrm{eq}}(t)$ is common to the two fluctuational paths that form the resultant corral (Dykman et al 1996a). From figure 12(c) we see that the ridges of the distribution are strongly asymmetric in time, but agree well with the fluctuational and relaxational paths predicted from (26).

Our third example (figure 13) is the system suggested by Maier and Stein (1993b, 1996a, 1997) in their analysis of the escape problem in non-equilibrium systems. It describes the motion of an overdamped particle in the two-dimensional drift field with the drift term of 
the form of $K_{1}\left(x_{1}, x_{2}\right)=\left(x_{1}-x_{1}^{3}-\alpha x_{1} x_{2}^{2},-x_{2}-x_{1}^{2} x_{2}\right)$, where $\alpha$ is a parameter. It has point attractors at $( \pm 1,0)$ and a saddle point at $(0,0)$. In describing the motion of the particle we will use the variables $x, y$ instead of $x_{1}, x_{2}$, as was done in the original work by Maier and Stein. If the particle is subject to additive isotropic white noise $f(t)$ its position on $(x, y)$-plane will satisfy the coupled Langevin equations

$$
\begin{aligned}
& \dot{x}=x-x^{3}-\alpha x y^{2}+f_{x}(t) \\
& \dot{y}=-y-x^{2} y+f_{y}(t) \\
& \left\langle f_{i}(t)\right\rangle=0 \quad\left\langle f_{i}(s) f_{j}(t)\right\rangle=D \delta_{i j} \delta(s-t) .
\end{aligned}
$$

Since $K$ is not a gradient field (unless $\alpha=1$ ), the dynamics will not satisfy detailed balance.

The circuit model of (46) is of some interest, not least because it demonstrates the ease with which the analogue technique may be applied to coupled equations. A block diagram is shown in figure 13: $f_{x}^{\prime}\left(t^{\prime}\right)$ and $f_{y}^{\prime}\left(t^{\prime}\right)$ are respectively the $x$ and $y$ components of the noise voltage applied to the circuit, and $t^{\prime}$ is the real time. The differential equations for the voltages appearing at points B and A in the circuit, in terms of the voltages $y\left(t^{\prime}\right)$ and $x\left(t^{\prime}\right)$ at the outputs of the corresponding operational amplifiers, are respectively

$$
\begin{aligned}
& C_{2} \frac{\mathrm{d} x}{\mathrm{~d} t^{\prime}}+\frac{1}{R_{6}}\left(x^{3}-x\right)+\frac{1}{R_{5}} x y^{2}+\frac{1}{R_{4}} f_{x}^{\prime}\left(t^{\prime}\right)=0 \\
& C_{1} \frac{\mathrm{d} y}{\mathrm{~d} t^{\prime}}+\frac{1}{R_{3}} y+\frac{1}{R_{2}} x y^{2}+\frac{1}{R_{1}} f_{y}^{\prime}\left(t^{\prime}\right)=0
\end{aligned}
$$

which can be rewritten as

$$
\begin{aligned}
& R_{4} C_{2} \frac{\mathrm{d} x}{\mathrm{~d} t^{\prime}}=-\frac{R_{4}}{R_{6}}\left(x^{3}-x\right)-\frac{R_{4}}{R_{5}} x y^{2}-f_{x}^{\prime}\left(t^{\prime}\right) \\
& R_{1} C_{1} \frac{\mathrm{d} y}{\mathrm{~d} t^{\prime}}=-\frac{R_{1}}{R_{3}} y-\frac{R_{1}}{R_{2}} x y^{2}-f_{y}^{\prime}\left(t^{\prime}\right) .
\end{aligned}
$$

If we choose circuit parameters

$R_{1}=R_{2}=R_{3}=R_{4}=100 \mathrm{k} \Omega \quad R=10 \mathrm{k} \Omega \quad C_{1}=C_{2}=10 \mathrm{nF}$

introduce a time constant $\tau=R_{1} C_{1}=R_{4} C_{2}$ and set the parameter $\alpha=R_{4} / R_{5}$, equations (48) reduce to

$$
\begin{aligned}
& \tau \frac{\mathrm{d} x}{\mathrm{~d} t^{\prime}}=x-x^{3}-\alpha x y^{2}-f_{x}^{\prime}\left(t^{\prime}\right) \\
& \tau \frac{\mathrm{d} y}{\mathrm{~d} t^{\prime}}=-y-x^{2} y-f_{y}^{\prime}\left(t^{\prime}\right)
\end{aligned}
$$

whose parameters are readily related to those in the model (46) by means of the scaling relations

$$
t=\frac{t^{\prime}}{\tau} \quad f(t)=-f^{\prime}\left(t^{\prime}\right)
$$

The circuit model was driven by two uncorrelated noises and its response was analysed using the Microstar data acquisition system described in section 2. The prehistory/posthistory probability distribution was measured in the usual way, except that the termination point was specified in terms of a small rectangle centred on $\left(x_{f}, y_{f}\right)$.

In measurements of the paths starting and ending in one and the same stable state, the lack of detailed balance and the asymmetry of the optimal fluctuational and relaxational 


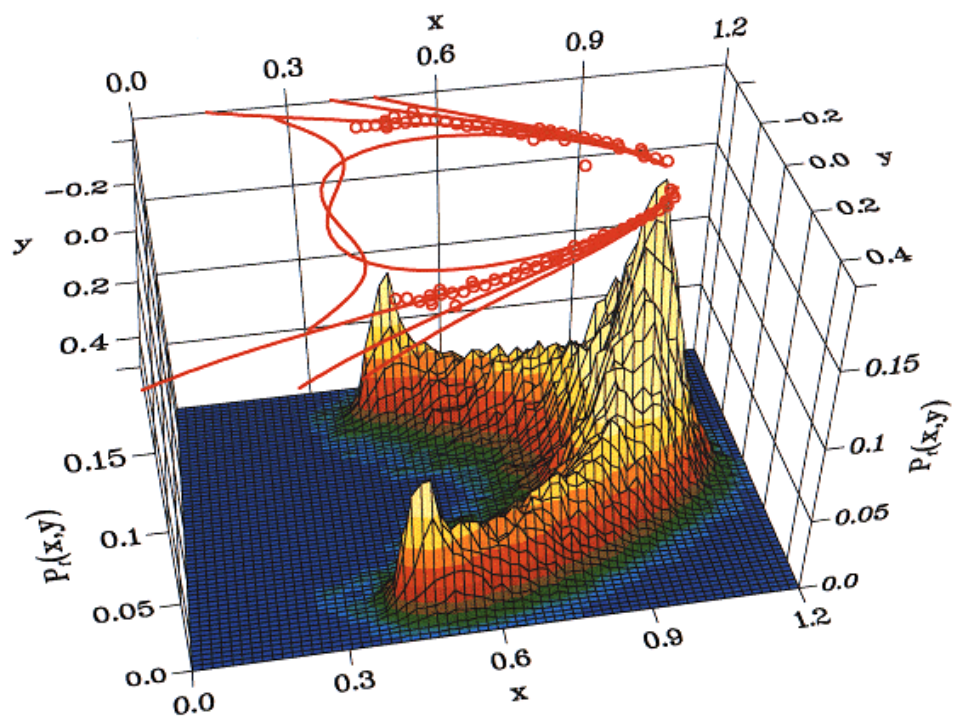

(a)

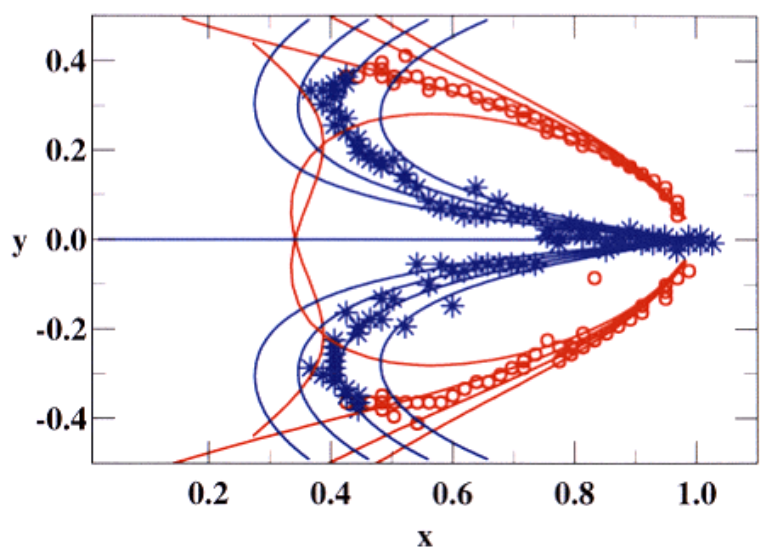

(b)

Figure 14. Fluctuational behaviour measured and calculated for the Maier and Stein nonequilibrium system (46), which has a stationary non-gradient field, with $a=10, D=0.014$. (a) The $p_{f}(x, y)$ distribution created by ensemble-averaging fluctuational paths leading from $S=(1,0)$ to remote points at $x_{f}=(0.44 \pm 0.35)$. (b) Paths traced out by the ridges of the distribution in (a) for fluctuational motion (red circles), and by those of the corresponding distribution for relaxational motion (blue asterisks), compared with fluctuational (red) and relaxational (blue) optimal paths calculated from (26). (c) As in (b), but for the single remote state $\boldsymbol{x}_{f}=(0.32,0)$ on the switching line (Luchinsky and McClintock 1997).

paths become strikingly apparent. Figure 14(a) shows the measured probability distribution $P_{f}(x, y)$ for fluctuations to the two different remote states $\left(x_{f}, y_{f}\right)$ placed symmetrically on either side of the $y$-axis; here, $P_{f}(x, y)$ is the integral over the time $t$ of the probability distribution $p_{f}\left(x, y, t ; x_{f}, y_{f}, t_{f}\right)$, i.e. the projection of the distribution $p_{f}$ onto the $x-y$ plane with $t_{f}=0$ as before. When the paths traced out by the ridges are plotted (figure 14(b)), it can be seen: (i) that the fluctuational trajectories are completely different 


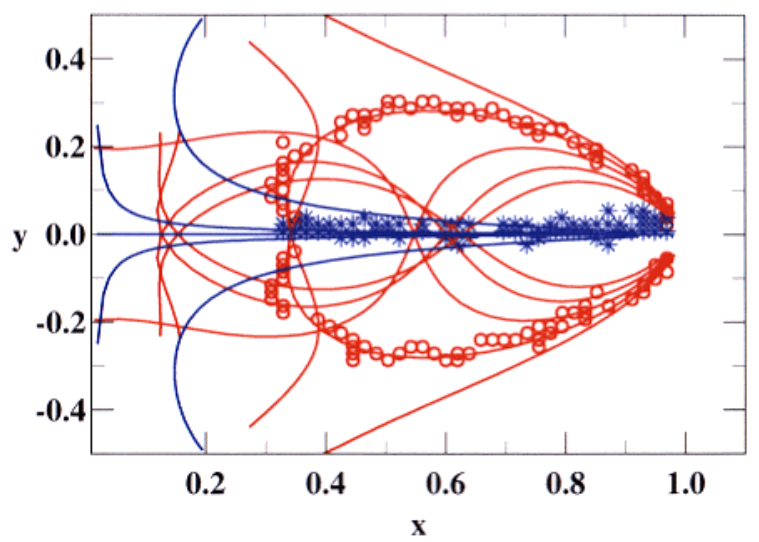

(c)

Figure 14. Continued.

from the relaxational ones; and (ii) that they are in good agreement with the fluctuational and relaxational trajectories predicted from (26). Figure 14(c) shows the corresponding picture measured for fluctuations to a single remote state $\boldsymbol{x}_{f}$ on the switching line (lying on the $x$-axis, by symmetry arguments). The two fluctuational paths to the remote state are, again, markedly different from the common relaxational path leading back to the stable state $(1,0)$. Both parts of the trajectory are well described by the corresponding optimal paths calculated from (26). In this case too, therefore, the observations link a lack of detailed balance to the temporal asymmetry of fluctuational and relaxational paths, and demonstrate the non-differentiability (Graham and Tél 1985) of the generalized nonequilibrium potential; the closed loops traversed during fluctuations verify the expected (Onsager 1931, Landau and Lifshitz 1980) occurrence of flows in the stationary state of non-equilibrium systems.

3.3.3. Singularities in the noisy exit problem and time symmetry-breaking. Analogue simulations have proven to be very effective as a tool for the investigation of escape rates and effective activation energies of escape in systems lacking detailed balance. Some results are discussed below in sections 4.3.1 and 6.2, including the results on a fundamentally important (Gabrielse et al 1985, Dehmelt 1990) periodically-driven nonlinear oscillator, which was essentially the first model of a physical system without detailed balance where escape rates were calculated explicitly (Dykman and Krivoglaz 1979). Singularities in the pattern of optimal paths of the driven oscillator were found numerically by Chinarov et al (1993), including the fact (later explained analytically (Dykman et al 1994e)) that caustics may emanate from unstable stationary states in systems lacking detailed balance.

Here we will present, in the context of an exit problem, recent results by Luchinsky et al (1997) on the relationships between the onset of focusing singularities and nonanalytic behaviour of an 'activation energy', broken time symmetry and lack of detailed balance, using as an example the system (46) considered by Maier and Stein (1993a, b, 1996a, 1997). This system is bistable, but because of the symmetry the escape rates are the same for each of the attractors $( \pm 1,0)$. In the weak noise limit, the escape rate is then given by the lowest non-zero eigenvalue $\lambda_{1}$ of the Fokker-Planck operator which corresponds to the Langevin equations (46) (Risken 1993). For small $D$ the eigenvalue $\lambda_{1}$ becomes exponentially small. 
In this limit the mean first passage time (MFPT) $\left\langle t_{\text {exit }}\right\rangle$ is well approximated by $\lambda_{1}^{-1}$. We note that the exponential slowness of the decay justifies application of the eikonal approximation and the prehistory formulation discussed above to the dynamics of large fluctuations about metastable states.

For weak noise intensity, the escape from a domain of attraction typically follows a unique trajectory. This most probable escape path (MPEP) corresponds to the heteroclinic Hamiltonian trajectory of the auxiliary system described by the equations (26). The trajectory starts from the fixed point that corresponds to the attractor of the initial system (the point $\boldsymbol{x}_{\mathrm{eq}}=( \pm 1,0), \boldsymbol{p}=\mathbf{0}$ for the (46) system) and goes to the fixed point which corresponds to the saddle point of the dynamical system $\left(\boldsymbol{x}_{\mathrm{s}}=\mathbf{0}, \boldsymbol{p}=\mathbf{0}\right.$ in the case of (46)). In the case of a time-independent Hamiltonian $H$, the motion occurs with zero energy.

The MPEP action $\delta W \equiv S\left(\boldsymbol{x}_{\mathrm{s}}\right)$ governs the weak noise behaviour of the MFPT. To leading order the MFPT is of the activation type, i.e.

$$
\left\langle t_{\text {exit }}\right\rangle \sim \text { constant } \times \mathrm{e}^{\delta W / D} \quad D \rightarrow 0 .
$$

So $\delta W$ is interpreted as an activation energy of escape (Dykman and Krivoglaz 1979).

In the system (46), when $\alpha=1$, the dynamics of the particle satisfy detailed balance, and the pattern of optimal trajectories emanating from $\left(\boldsymbol{x}_{\mathrm{eq}}, \boldsymbol{p}=\mathbf{0}\right)$ contains no singularities. It was found earlier (Maier and Stein 1993b, 1996a) that, as $\alpha$ is increased, the first focusing singularity on the MPEP (initially lying along the $x$-axis) appears when $\alpha=\alpha_{\mathrm{c}} \equiv 4$. It signals the appearance of a transverse 'soft mode,' or instability, which causes the MPEP to bifurcate. Its physical origin is clear: as $\alpha$ is increased, the drift toward the attractor $(1,0)$ 'softens' away from the $x$-axis, which eventually causes the on-axis MPEP to split. The two new MPEPs move off-axis, causing the activation energy (previously constant) to start decreasing. So the activation energy as a function of $\alpha$ is non-analytic at $\alpha=\alpha_{\mathrm{c}}$ (Maier and Stein 1996a).

To test these theoretical predictions Luchinsky et al (1997) have investigated escape events in an analogue electronic model of the system (46) (see figure 13). Transition probabilities were measured by a standard level-crossing technique. Experimental investigations of the optimal fluctuational and relaxational trajectories were based on the experimental technique described in the previous section. Luchinsky et al (1997) have also carried out a complementary digital simulation of (46) using the algorithm of Mannella (1997).

Some activation energy results are shown in figure 15. Part (a) plots the MFPT $\left\langle t_{\text {exit }}\right\rangle$ as a function of inverse noise intensity $1 / D$ for the special case $\alpha=1$. In this case, the drift field is the gradient of the potential $U(x, y)=\left(y^{2}\left(1+x^{2}\right)-x^{2}+x^{4} / 2\right) / 2$, and $\delta W$ can be obtained exactly $(\delta W=2 U)$. The analogue and digital results are in good agreement, and demonstrate that the noise dependence of the MFPT is indeed of the activation type predicted by the theory (see also section 4.1 where the results for a different system lacking detailed balance are discussed). Activation energies determined from the slopes of a series of plots like those in figure 15(a), yielded the results shown in figure 15(b), where they are compared with theoretical values of $\delta W$ determined from the true (least action) MPEP or MPEPs (Maier and Stein 1993b, 1996a). At the predicted critical value $\alpha_{\mathrm{c}}=4$, marked changes in the activation energy are evident.

To verify experimentally the expected relationship between the bifurcation of the MPEP and the bifurcation of the activation energy, Luchinsky et al (1997) have measured 2D prehistory probability distributions of fluctuational trajectories bringing the system into the vicinity of the separatrix between the two wells (the $y$-axis). In the limit of low noise intensity, the maxima of the corresponding distributions trace out optimal trajectories. 

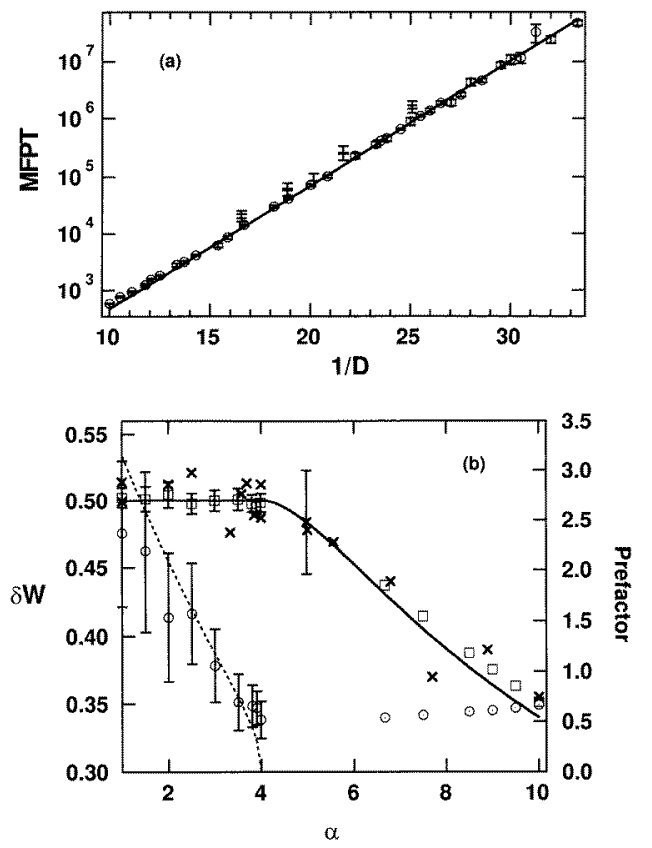

Figure 15. (a) The mean first passage time as a function of inverse noise intensity $1 / D$ for $\alpha=1$, from analogue experiment (bars), numerical simulation (circles) and calculation (full line). (b) The inter-well activation barrier $\delta W$, as a function of $\alpha$, from analogue experiment (crosses), numerical simulation (squares) and theory (full curve). The variation of the prefactor with $\alpha$ (circles and dotted curve) is discussed in the original paper (Luchinsky et al 1997).

The positions of these maxima are compared to the calculated MPEPs for $\alpha=6.67$ in figure 16(a). It is clear that the typical fluctuational path corresponding to escape from the domain of attraction of $\left(x_{s}, 0\right)$ follows very closely one of the predicted MPEPs.

To seek further experimental insight into the character of the broken symmetry for the MPEP, Luchinsky et al (1997) have also followed the dynamics of the relaxational part of the escape paths, after they have crossed the $y$-axis separatrix (in the general case, only one side of the separatrix can be reached along optimal paths coming out of a given stable state (Dykman et al 1994e)). The positions of the maxima of the measured relaxational distributions are compared with the corresponding theoretical trajectories in figure 16(b). It can be seen from the figure that for $\alpha>\alpha_{\mathrm{c}}$ the MPEP breaks time-reversal symmetry in the sense that the average growth and average decay of fluctuations (Onsager 1931) traced out by the ridges of the corresponding distributions take place along trajectories that are asymmetric in time. That is, for $\alpha>\alpha_{c}$ the MPEP is not a time-reversed relaxational trajectory.

The inset in figure 16(b) shows the distribution of points where the escape trajectories hit the $y$-axis separatrix (i.e. the exit location distribution). Its shape is nearly Gaussian, as expected from the saddle-point approximation of Maier and Stein (1996a, 1997). The maximum is situated near the saddle point clearly demonstrating that, in the limit of weak noise, exit occurs via the saddle point - a result which has been obtained independently by several groups (see Matkowsky and Schuss 1983, Freidlin and Wentzell 1984, Dykman and Krivoglaz 1984, Day 1987 and references in these papers; cf Maier and Stein 1996b). 


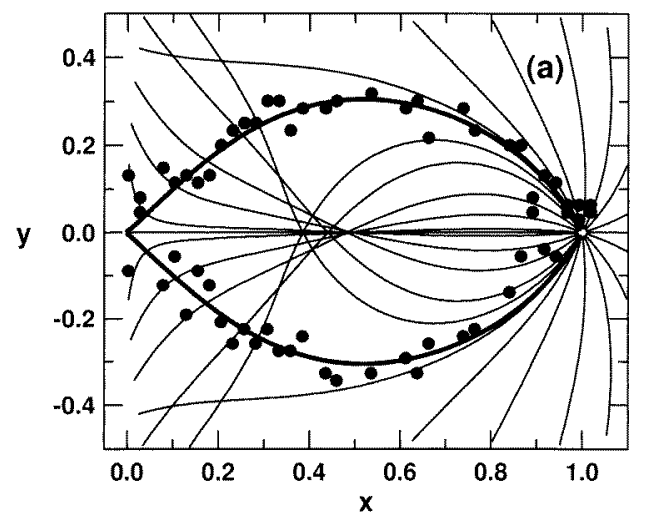

(a)

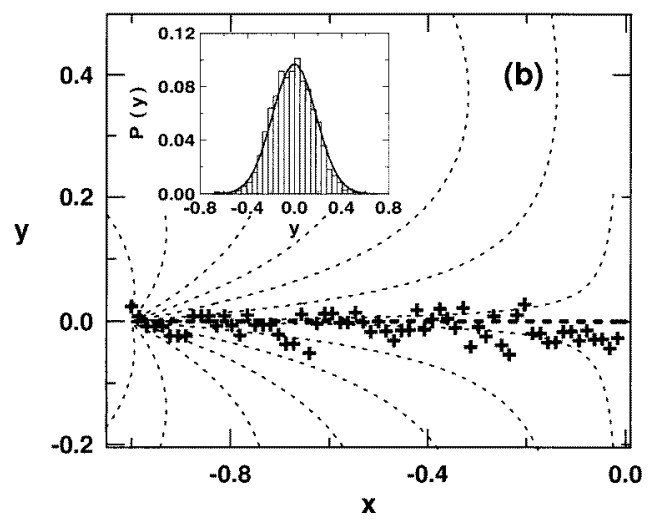

(b)

Figure 16. Measured positions of the ridges (first moments) for $\alpha=6.67$ of (a) the fluctuational part (full circles) and (b) the relaxational part (pluses) of the escape trajectories. Nearby theoretical trajectories are shown by curves. The inset shows the exit location distribution along the $y$-axis (Luchinsky et al 1997).

We note that, because of the symmetry $y \rightarrow-y$ of the Maier and Stein system (46), the MPEP $y_{\text {opt }}=0$ can remain unchanged up to the value $\alpha_{\mathrm{c}}=4$, whereas the system lacks detailed balance whenever $\alpha \neq 1$. Thus, for $1<\alpha<4$ the dynamics of the most probable fluctuational trajectories is a mirror image of the relaxational dynamics only along the $x$-axis; everywhere else in the domain of attraction of $( \pm 1,0)$ the outward optimal trajectories are not antiparallel to the inward relaxational trajectories, and the resulting loops enclose non-zero area (Freidlin and Wentzel 1970).

This prediction has been tested experimentally (Luchinsky et al 1997) by tracing out optimal paths to/from specified remote states both on and off the $x$-axis, for $1<\alpha<\alpha_{\text {c }}$. Some results are shown in figure 17 for $\alpha=3.5$. It is evident that the ridges of the fluctuational (full circles) and relaxational (pulses) distributions follow closely the theoretical curves. For an off-axis remote state (figures 14(a) and (b), 17(a)), they form closed loops of non-zero area, thus demonstrating again the expected rotational flow of the probability current in a non-equilibrium system (Onsager 1931). The corresponding ridges for an on- 


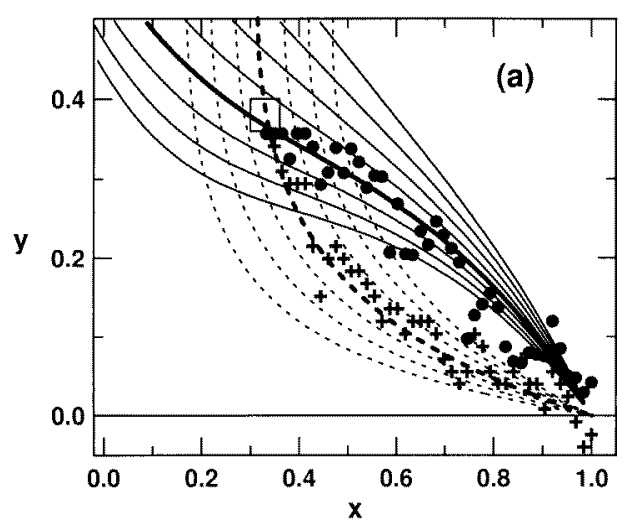

(a)

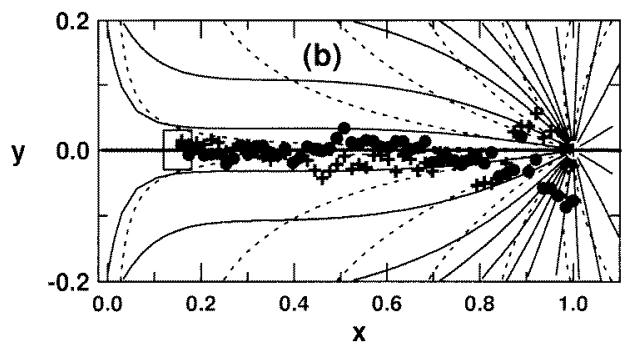

(b)

Figure 17. Demonstration of the 'local' character of detailed balance and time-reversal symmetry of (46) for $\alpha=3.5$. (a) Measured positions of the ridges of the fluctuational (full circles) and relaxational (pluses) parts of the trajectories from $(1,0)$ to $(0.3,0.3)$, compared with theoretical predictions (curves) by Maier and Stein (1993a, 1996a). (b) The same for trajectories extending to the on-axis remote state $(0.1,0)$ (Luchinsky et al 1997).

axis remote state (figure 17(b)) are antiparallel, indicating that symmetry is preserved along the $x$-axis.

Luchinsky et al (1997) conclude that these results have demonstrated that, in the limit $D \rightarrow 0$, detailed balance and time-reversal symmetry can be considered as local properties along the MPEP of the system in the sense discussed above, and that the bifurcation phenomenon can be related to local time-reversal symmetry breaking along the MPEP: results that may be important for 2D stochastic ratchets (e.g. Slater et al 1997) where symmetry plays an important role.

\subsection{Status of the experiments on large fluctuations}

The results described above in this section, as well as those discussed in sections 4.1 and 6 show that, after so many years of investigating the probabilities of large fluctuations, it has now become possible to use the prehistory formulation as a basis for experiments on the fluctuation dynamics. The work on Markov systems presented above has already verified several long-standing theoretical predictions, including the very observability of optimal fluctuational paths, narrowness of the tube of fluctuational paths, symmetry between the growth and decay of classical fluctuations in equilibrium Markov systems (Onsager 1931, Onsager and Machlup 1953, Landau and Lifshitz 1980), the breaking of this symmetry under 
non-equilibrium conditions where systems lack detailed balance (Ventcel' and Freidlin 1970, Dykman and Krivoglaz 1979, Jauslin 1987a b, Maier and Stein 1993a, 1997), the relationship of the lack of detailed balance to the onset of singularities in the pattern of optimal paths, as well as the character of these singularities (Dykman et al 1994e, Maier and Stein 1996a, b, Dykman et al 1996c, Smelyanskiy et al 1997a), including occurrence of switching between optimal paths and critical broadening of the paths distribution.

In fact, the very possibility of experimental observation of fluctuation dynamics was crucial for stimulating theoretical interest, and in the last decade it has made fluctuation dynamics an active area of theoretical and experimental efforts, with many novel effects yet to be explored, and with various possible applications. We will mention further research (Luchinsky et al 1998) on the important problem of escape from a metastable state for the Maier and Stein system (46), investigations of the dispersion of the prehistory distribution and the conditions under which it is non-monotonic (Arrayas et al 1998a), studies of the possibility (Smelyanskiy and Dykman 1997, Vugmeister and Rabitz 1997) of highly effective control of large fluctuations and work on how to control the rate and direction of diffusion in periodic systems (Arrayas et al 1997, 1998b).

\section{Stochastic resonance}

Stochastic resonance (SR) has been much in the news recently, partly on account of its wide occurrence in many areas of science. In this section, we first present a very succinct introductory review of SR - which, as already mentioned, was first measured experimentally in an analogue electronic circuit (Fauve and Heslot 1983) - and then describe how subsequent electronic experiments have made major contributions to the development of a mature understanding of SR and of several related phenomena.

\subsection{What is stochastic resonance?}

Stochastic resonance (SR) is commonly said to occur when a weak periodic signal in a nonlinear system is enhanced by an increase of the ambient noise intensity; a stronger definition requires that the signal-to-noise ratio (SNR) should also increase. The usual observation is that the signal amplitude increases with increasing noise intensity, passes through a maximum and then decreases again. Thus, the general behaviour is somewhat similar to a conventional resonance curve, but the response is plotted as a function of noise intensity instead of frequency. The SNR typically displays very similar behaviour except that it falls very rapidly before the onset of the counter-intuitive increase; in the case of systems with thresholds, or two-state systems (or where the signal from a continuous system is passed through a two-state filter prior to analysis), this initial decrease in the SNR is not seen. In the strongly nonlinear (large signal/weak noise) regime, the variation of the response with noise intensity can be more complicated (see later).

The SR phenomenon appears to be widespread. After being introduced as a possible explanation of the earth's ice-age cycle (Benzi et al 1981, Nicolis 1982), SR has subsequently been observed or invoked in contexts that include lasers (McNamara et al 1988, Vemuri and Roy 1989, Fioretti et al 1993, Iannelli et al 1994), passive optical systems (Dykman et al 1991c, Grohs et al 1994, Jost and Saleh 1996, Vaudelle et al 1998), tunnel diodes (Mantegna and Spagnolo 1994), a Brownian particle in an optical trap (Simon and Libchaber 1992), a magnetoelastic ribbon (Spano et al 1992), crayfish and rat mechanoreceptors (Douglass et al 1993, Collins et al 1996), a bistable SQUID (superconducting quantum interference device) (Hibbs et al 1995), arrays of SR elements 
(Lindner et al 1995), ion channels (Bezrukov and Vodyanoy 1995), magnetic systems (Grigorenko et al 1997), various types of electronic models (e.g. Fauve and Heslot 1983, Gammaitoni et al 1989. Dykman et al 1990c, d, Anishchenko et al 1993, 1994), including models of coexisting periodic attractors (Dykman et al 1993b, 1996a), subcritical bifurcations (Vohra and Bucholtz 1993), systems with threshold (Gingl et al 1995), and transient dynamics (Iwaniszewski 1995), a quantum two-level system (Lofstedt and Coppersmith 1994), an array of coupled bistable systems (Morillo et al 1995, Bulsara and Gammaitoni 1996), a system driven by quasimonochromatic (harmonic) noise (Neiman and Schimansky-Geier 1994), excitable neurons (Longtin 1995) and chemical systems (Leonard and Reichl 1994, Dykman et al 1995c). There have recently been two topical conferences (Moss et al 1993, Bulsara et al 1995), several general scientific articles (Wiesenfeld 1993, Moss and Xing Pei 1995, Moss and Wiesenfeld 1995, Bulsara and Gammaitoni 1996) and topical reviews (Jung 1993, Moss 1994, Wiesenfeld and Moss 1995, Dykman et al 1995d, e, 1996b, Gammaitoni et al 1998), including one (Dykman et al 1995d) that specifically attempts to place SR in its scientific and historical context, and to connect it to related phenomena in physics.

In bistable systems, the underlying mechanism of SR is easily appreciated, and in fact has been known since the work by Debye (1929) on reorienting polar molecules. In a static double-well potential with equally-deep wells, the effect of an additive low-frequency periodic force is to tilt the wells first in one direction, and then in the other, so that one of the wells become deeper than the other, in turn. The effect of additive noise is, on average, to induce fluctuational transitions to the deeper well. For very weak noise intensity, there will on average be no transitions within a half cycle of the periodic force; for very strong noise intensity the directions of transitions will be random, virtually unaffected by the periodic force; and for an optimum noise intensity, the probability of such transitions occurring coherently twice (once in each direction) per full cycle of the periodic force will be maximized. Thus noise can effectively amplify small coordinate variations within one of the potential wells, caused directly by the rocking effect of the periodic force, to an amplitude corresponding to the coordinate separation of the potential minima-which can be made large, resulting in a correspondingly large susceptibility, and frequency dispersion (Debye 1929).

SR was originally discussed (Benzi et al 1981, Nicolis 1982) for a bistable system and, in the following years, it was widely assumed that bistability was an essential prerequisite for the phenomenon to occur. The description of SR as a linear response phenomenon (see section 4.2) led naturally, however, to the realization that SR can also occur without bistability (Stocks et al 1992) and to the observation of the phenomenon in an underdamped, monostable, nonlinear oscillator (Stocks et al 1993c). In fact, it is well known that the response of a monostable system to signals in certain frequency ranges can be strongly increased by noise, for example just by raising the temperature. Examples range from currents in electron tubes to optical absorption near absorption edges in semiconductors. For underdamped oscillators, a temperature-induced shift and broadening of the absorption peaks, i.e. 'tuning' by external driving due to the oscillator nonlinearity, was first discussed by Ivanov et al (1966); complete classical and quantum theories of these effects were given by Dykman and Krivoglaz (1984).

Some non-conventional forms of SR, including monostable SR, were reviewed by Dykman et al (1993d). Since then, forms of SR without bistability have been identified in a system with a cyclic variable (Wiesenfeld et al 1994), a class of systems where the signal is applied as a multiplicative force (Vilar and Rubi 1996) and thresholdless systems (Bezrukov and Vodyanoy 1997). SR in a monostable SQUID model has recently been shown (Kaufman 
et al 1996) to meet a fortiori even the stronger of the above definitions: the observed noiseinduced SNR enhancements were comparable both in magnitude and form with those of conventional SR. We consider monostable SR in more detail in section 4.3.2.

Throughout the development of the subject area, analogue electronic experiments have played a major role. We have already cited a few of the key papers, and will refer to some more of them below; for a fuller bibliography, reference should be made to the review articles on SR and the conference proceedings already mentioned.

\subsection{Stochastic resonance as a linear response phenomenon}

When it was first discovered, and for some time afterwards, SR seemed a rather mysterious phenomenon and a number of highly sophisticated theoretical approaches were proposed (see citations in, for example, the reviews by Jung 1993, Moss 1994, Wiesenfeld and Moss 1995). Only some years later was it appreciated (Dykman et al 1990c, d, Gammaitoni et al 1998) that a much simpler formalism—linear response theory (LRT)—would suffice to describe what was often the most interesting limit in practice, where the signal was relatively small; an analytic theory of the more complicated effects that occur for stronger signal strengths (Dykman et al 1990c, 1993f, Zhou et al 1990, Stocks 1995) has also been developed, and has been confirmed in considerable detail through analogue electronic experiments. LRT has the particular advantage, however, that it places SR in perspective and enables it to be understood in the context of other more familiar phenomena in physics (Dykman et al 1995d). It therefore provides the best starting point for understanding SR.

If a system with a coordinate $x$ is driven by a weak force $A \cos \Omega t$ then, according to LRT (Landau and Lifshitz 1980), a small periodic term will appear in the ensemble-averaged value of the coordinate $\delta\langle x(t)\rangle$, oscillating at the same frequency $\Omega$ and with amplitude $a$ proportional to that of the force:

$$
\begin{aligned}
& \delta\langle x(t)\rangle=a \cos (\Omega t+\phi) \equiv \operatorname{Re}\left[\chi(\Omega) A \mathrm{e}^{-\mathrm{i} \Omega t}\right] \quad A \rightarrow 0 \\
& a=A|\chi(\Omega)| \quad \phi=-\arctan [\operatorname{Im} \chi(\Omega) / \operatorname{Re} \chi(\Omega)] .
\end{aligned}
$$

This equation holds for dissipative and fluctuating systems that do not display persistent periodic oscillations in the absence of the force $A \cos \Omega t$ and where the correlations of fluctuations decay in time. The susceptibility $\chi(\Omega)$ contains all information on the response of the system to a weak driving force. It gives both the amplitude of the signal, $a$, and its phase lag $\phi$ with respect to the force (and the partial amplitudes and phase lags for vibrations at the combination frequencies). Equation (52) can be easily generalized to the case where the force is of a more general nature than just an additive coordinate-independent force described by an extra term $-A x \cos \Omega t$ in the Hamiltonian. In particular, we consider below cases where the force is coordinate dependent (a multiplicative force), or where it is the intensity of the noise driving the system (e.g. the temperature, if the noise is of thermal origin) that is modulated periodically. Provided that the amplitude of the modulation is weak enough, the response of the system will always be linear and described by (52).

The periodic term (52) induced by the force gives rise to a $\delta$-shaped spike in the spectral density of fluctuations (SDF)

$$
Q(\omega)=\lim _{\tau \rightarrow \infty}(4 \pi \tau)^{-1}\left|\int_{-\tau}^{\tau} \mathrm{d} t x(t) \exp (\mathrm{i} \omega t)\right|^{2}
$$

at the frequency $\Omega$ of the force. The intensity (i.e. the area) of this spike is equal to one fourth of the squared amplitude of the corresponding vibrations, i.e. to $\frac{1}{4} A^{2}|\chi(\Omega)|^{2}$. The 
signal-to-noise ratio $R$ can thus be written

$$
R=\frac{1}{4} A^{2}|\chi(\Omega)|^{2} / Q^{(0)}(\Omega) \quad(A \rightarrow 0)
$$

where $Q^{(0)}(\omega)$ is the SDF in the absence of the periodic driving. The evolutions of the susceptibility and of $Q^{(0)}(\omega)$ with varying noise intensity $D$ therefore show immediately whether or not SR (in terms of the strong definition, as an increase in SNR with increasing $D$ in a certain range of $D$ ) is to be expected at a given frequency.

Describing SR in terms of a susceptibility in this way is particularly advantageous for systems that are in thermal equilibrium, or in quasi-equilibrium. In such cases the fluctuation-dissipation relations (Landau and Lifshitz 1980) can be used to express the susceptibility in terms of $Q^{(0)}(\omega)$

$\operatorname{Im} \chi(\omega)=\frac{\pi \omega}{D} Q^{(0)}(\omega) \quad \operatorname{Re} \chi(\omega)=\frac{2}{D} \mathrm{P} \int_{0}^{\infty} \mathrm{d} \omega_{1} Q^{(0)}\left(\omega_{1}\right) \frac{\omega_{1}^{2}}{\omega_{1}^{2}-\omega^{2}}$

where $\mathrm{P}$ denotes the Cauchy principal value and $D$ corresponds to the temperature in energy units. It follows from (54) and (55) that it should be possible to predict the onset of SR in a given system purely from the evolution of its $\operatorname{SDF} Q^{(0)}(\omega)$ with noise intensity (temperature), without knowing or assuming anything at all about the equations that describe its dynamics, i.e. for a system treated as a 'black box'. The susceptibility can also be obtained by analytic solution of the Fokker-Planck equation (Dykman et al 1993a), leading to an identical result.

These ideas were first tested (Dykman et al 1990c, d) through an analogue simulation of underdamped one-dimensional Brownian motion

$$
\begin{aligned}
& \ddot{x}+2 \Gamma \dot{x}+\frac{\partial U}{\partial x}=f(t)+A \cos (\Omega t) \\
& \langle f(t)\rangle=0 \quad\left\langle f(t) f\left(t^{\prime}\right)\right\rangle=4 \Gamma D \delta\left(t-t^{\prime}\right)
\end{aligned}
$$

in the simple symmetric bistable potential (30). The circuit model was very similar to that of figure 2, except that the $V_{2}$ term was not inverted before being fed back to point A. Two different kinds of measurements were made on the system. First, SDFs were measured for several values of $D$ with the weak periodic force applied $(A \neq 0)$, and the SNR was determined through measurements of the ratio of the height of the spike at $\Omega$ to that of the noisy background (Fauve and Heslot 1983). These direct measurements of the SNR are shown as square data points in figure 18. Secondly, SDFs were measured in the absence of the periodic force $(A=0)$ for several different noise intensities. These results were used to compute the SNR to be expected on from the LRT relations (54) and (55) which are shown as 'pluses' in the figure. The agreement between the theory and the direct measurement is satisfactory, within the scatter of the data. Given that there are no adjustable parameters, it may be considered an impressive vindication of the LRT approach to SR. Of course, the SDF $Q^{(0)}(\omega)$ for insertion in (54) and (55) does not necessarily need to be measured, as here. It can also be calculated analytically over a wide range of parameters (Dykman and Krivoglaz 1984, Dykman et al 1988, 1989).

Note that the onset of SR in a bistable system can be understood in terms of the evolution with $D$ of its SDF and, in particular, the broadening of the zero-frequency peak (Beasley et al 1983, Dykman et al 1988, 1989) associated with the interwell fluctuational transitions. As the peak broadens $Q^{(0)}(\Omega)$, for $\Omega$ finite but small, rises exponentially fast. Thus from (55), it is clear that both $\operatorname{Re} \chi(\omega)$ and $\operatorname{Im} \chi(\omega)$ for $\omega=\Omega$ will also increase exponentially fast. It is clear from (52) and (54) that $a$ and $R$ will also both increase with $D$. This perception of bistable SR is, of course, a counterpart of the picture of noiseenhanced hopping between the wells in response to the external driving, which was put 


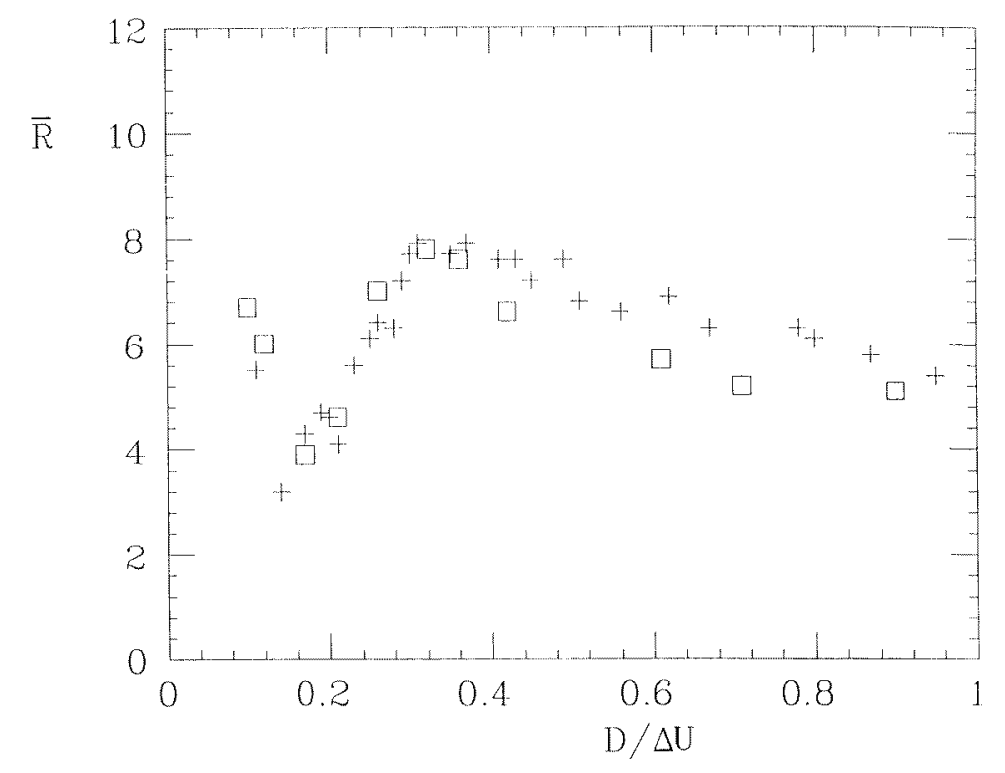

Figure 18. A demonstration (Dykman et al 1990c) that stochastic resonance can be described in terms of linear response theory (LRT). The squares represent direct measurements of the scaled signal-to-noise ratio $\bar{R}$ for the system (30) and (56); the pluses represent LRT calculations based on measured spectral densities and the use of (54) and (55).

forward by Debye (1929). Clearly, the LRT approach is not limited to systems in a static bistable potential, or to bistable systems in general, for that matter. And indeed, as we will see later, these ideas led directly to the prediction and observation of SR in monostable systems.

Surprisingly, perhaps, the obvious advantages of LRT in relation to SR-its simplicity, beauty and universality-took some time to win general acceptance. So a supplementary test of the LRT picture was performed through direct measurements and calculations (Dykman et al 1992b) of the phase shift $\phi$ between the periodic force and the system's response. The system chosen for study was the overdamped double-well Duffing oscillator, i.e. essentially the same as (56) but with the inertial term missing

$$
\begin{aligned}
& \dot{x}+\frac{\partial U}{\partial x}=f(t)+A \cos (\Omega t) \\
& \langle f(t)\rangle=0 \quad\left\langle f(t) f\left(t^{\prime}\right)\right\rangle=2 D \delta\left(t-t^{\prime}\right)
\end{aligned}
$$

where $U(x)$ is again the quartic bistable potential (30). The circuit model used was similar to that of figure 5(a). The phase shift $\phi$ was determined by measuring ensemble averages $\langle q(t)\rangle$ and comparing the resultant sinusoid directly with the weak periodic driving force $A \cos (\Omega t)$. Results are shown by the data points of figure 19. It can be seen that, as $D$ is increased, $-\phi$ starts from a very small value, rises extremely fast, passes through a maximum and then decreases more slowly again. The prediction of earlier theories (Nicolis 1982, McNamara and Wiesenfeld 1989, Presilla et al 1989), based on the two-state approximation, is shown by the dashed curve. A theory able to describe the continuous system under consideration is readily constructed (Dykman et al 1992b) on the basis of LRT. It leads to explicit expressions for the phase shift and SNR $R$ that are valid for relatively 
low noise intensities and driving frequencies $\Omega$

$$
\begin{aligned}
\phi & =-\arctan \frac{\left(\Omega / \Omega_{\mathrm{r}}\right)\left(\Omega_{\mathrm{r}}^{2} W+\Omega^{2} D\right)}{\Omega_{\mathrm{r}} W^{2}+\Omega^{2} D} \\
R & =\frac{\pi A^{2}}{4 D^{2}} \frac{\Omega_{\mathrm{r}}^{2} W^{2}+\Omega^{2} D^{2}}{\Omega_{\mathrm{r}}^{2} W+\Omega^{2} D} \quad \Omega, D \ll \Omega_{\mathrm{r}} \quad W \ll D
\end{aligned}
$$

where $W$ is the inter-well transition probability in the absence of periodic forcing, and the reciprocal relaxation time $\Omega_{\mathrm{r}}=U^{\prime \prime}(x= \pm 1)=2$. For very small $D$, where $W \ll\left(\Omega^{2} / \Omega_{\mathrm{r}}^{2}\right) D$, it follows from (59) that $R \simeq \pi A^{2} / 4 D, \phi \simeq-\Omega / \Omega_{\mathrm{r}}$. Thus for a fixed forcing frequency $\Omega, R$ decreases with increasing $D$, whereas $\phi$ remains small and nearly independent of $D$. For larger values of $D$, it is straightforward to demonstrate that (59) implies that $R$ will pass through a minimum and then increase again (i.e. onset of the SR phenomenon) until $D \sim \Delta U / 2$, when the theory is no longer applicable (see the full curve in the inset to figure 19) because the interwell transitions cease to be distinct events. We would comment that the failure of the theory at large $D$ occurs because the expressions used for $Q^{(0)}(\omega)$ then become poor approximations; it is not a failure of LRT as such. We would also comment that the LRT predictions of an initial fall in $R$ with increasing $D$ and of the existence of the minimum in $R$ have been observed in many experiments, and were remarked upon and treated in an ad hoc fashion by McNamara and Wiesenfeld (1989) but have not been accounted for quantitatively by any theory of SR other than LRT (Dykman et al 1990c, d).

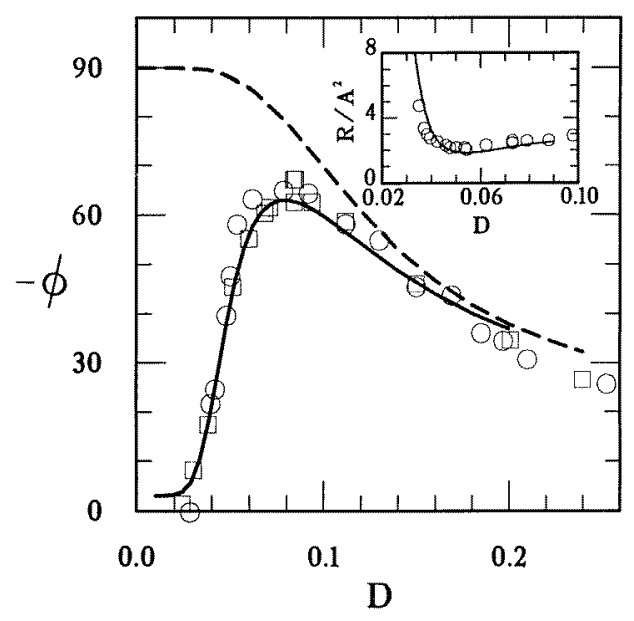

Figure 19. The phase shift $-\phi$ (degrees) between the periodic force of amplitude $A$ and the averaged coordinate $\langle x(t)\rangle$ measured (Dykman et al 1992b) as a function of noise intensity $D$ in the electronic model of (30) and (57) for $\Omega=0.1$ and: $A=0.04$ (circles); $A=0.2$ (squares). The full curve is a theoretical prediction based on LRT and the fluctuation dissipation theorem; the dashed curve represents the prediction (60) of (two-state) theories (Nicolis 1982, McNamara and Wiesenfeld 1989, Presilla et al 1989) that make no explicit allowance for the effect of intra-well vibrations. The inset compares experiment and LRT prediction for the normalized signal-to-noise ratio in the region of the minimum in $R$.

In agreement with equation (58), $|\phi|$ rises steeply from its $D \rightarrow 0$ value, passes through a maximum at $D=D_{\max }<\Delta U$, and then decreases again with further increase of $D$. For the parameters used in the experiment, a maximum value of $-\phi=68^{\circ}$ is predicted to occur at $D_{\max }=0.08$, which is to be compared with the experimental observation for $A=0.04$ 
of $-\phi=(66 \pm 2)^{\circ}$ at $D=0.08 \pm 0.01$. In accordance with the LRT prediction, the decrease of $|\phi|$ for $D>D_{\max }$ is much more gradual than the rapid increase seen below $D_{\max }$. The measured $\phi$ is relatively insensitive to $A$. The LRT prediction for the effect of noise on the phase shift, based on the full set of equations for $Q^{(0)}$ (Dykman et al 1992b), which goes beyond the range specified in (58), (59), is shown by the full curve in figure 19. It is evident that the analogue results are in excellent agreement with the theory. This form of behaviour was also confirmed by Morillo and Gómez-Ordóñez (1993) by numerical solution of the Fokker-Planck equation.

It is reasonable to wonder why the variation of $\phi$ with $D$ for SR in model (1) should be non-monotonic (figure 19), whereas the corresponding variation of $\phi$ with $\Omega$ in a deterministic resonance is well known to be monotonic, as also were the earlier predictions (Nicolis 1982, McNamara and Wiesenfeld 1989, Presilla et al 1989) for SR. An answer is readily inferred by physical intuition. For very small $D \ll \Delta U$, where the system is effectively confined to a single well, we may expect $\phi$ to be small because $\Omega$ is small compared with the reciprocal characteristic time of intra-well motion-for very large $D \gg \Delta U$, where the double-well character of the potential has become irrelevant, we may also expect $\phi \simeq 0$, for the same reason-so, at the intermediate values of $D$ where SR occurs, any significant phase lag associated with the SR must inevitably give rise to a maximum in $|\phi|$, just as observed. Earlier theories failed to predict this behaviour because, unlike the theory described above, they are effectively two-state treatments that take no account of the intra-well vibrations. This is easily shown (Dykman et al 1992b) because, if the intra-well vibrations are ignored, the expression for the phase shift (58) immediately reduces to the original Nicolis (1982) result

$$
\phi=-\arctan (\Omega / W)
$$

shown by the dashed curve in figure 19 .

Observation of the phase lags, as described here and by Gammaitoni et al (1991a,b), strengthens the analogy between bistable SR and conventional forms of resonance. We would stress, however, that it is only an analogy in the case of (57) because, although a description of SR in terms of a residence time distribution enables some resonancelike behaviour to be explored (Gammaitoni et al 1995), it remains true (Fox 1989) that there is no matching of $\Omega$ to any internal vibrational frequency of the system. This is to be contrasted with SR in underdamped monostable systems (Stocks et al 1992, 1993c) discussed in section 4.3.2, which is a true resonance phenomenon where external noise is used to tune the natural oscillation frequency of the system to that of the periodic force.

Digital simulations (Dykman et al 1993g) have shown that LRT also provides a satisfactory description of the system behaviour even when $\Omega$ has become so large that the SR effect has disappeared, and $R$ just decreases monotonically with increasing $D$. LRT has both advantages and disadvantages, as compared to other theoretical treatments. The main disadvantage is that it will only yield quantitatively accurate results when $A$ is small enough for the system to be within its regime of linear response; but this is, of course, a condition that is often fulfilled in practice. It should be noted, however, that the quality of the results obtained will naturally depend on the accuracy of the $Q^{(0)}(\omega)$ used in (55) for the calculation of $\chi(\Omega)$.

We now address the vexed question of whether an SR-displaying system (a 'stochastic resonator') can improve the SNR of a given signal. There has been much confusion on this point in the literature, with more than one research group claiming that absolute SNR improvements have been observed in experiments. However, Dykman et al (1995d) and DeWeese and Bialek (1995), using different LRT-based arguments, have pointed out that, 
for small-amplitude signals, the SNR at the output of a system driven by a stationary Gaussian noise does not exceed that at the input, even if the system displays SR. Indeed, the Fourier components of the noise are statistically independent and the total power of the noise $\Xi(\Omega) \mathrm{d} \Omega$ in a small spectral interval $\mathrm{d} \Omega$ about the frequency of the signal $\Omega$ is small. The signal-to-noise ratio at the input is given by $\frac{1}{4} A^{2} / \Xi(\Omega)$, whereas that at the output is $\frac{1}{4}|\chi(\Omega)|^{2} A^{2} /\left[|\chi(\Omega)|^{2} \Xi(\Omega)+Q^{\prime(0)}(\Omega)\right]$. The quantity $Q^{\prime(0)}(\Omega)$ gives the value of the spectral density of fluctuations in the system at frequency $\Omega$ as it would be if there was no signal and the spectral components of the noise at frequency $\Omega$ were suppressed, i.e. as if the power spectrum of the input noise had a hole at frequency $\Omega$. By construction $Q^{\prime(0)}(\Omega) \geqslant 0$, which proves the statement. (In linear systems, on the other hand, which do not mix frequencies, $Q^{\prime(0)}(\Omega)=0$ and the signal-to-noise ratio at the output must be the same as at the input.) These points deserve, and have recently received (Dykman and McClintock 1998), emphasis, given the level of misinformation in some of the recent literature on SR.

The situation with large-amplitude signals (beyond the LRT range) is less clear. Generally, such signals are distorted by a nonlinear system: the response to a sinusoidal signal has overtones, and therefore may not be characterized by the SNR at the signal frequency. In some models the SR effect decreases with signal amplitude for large signals (Jung and Hänggi 1991), whereas for specific types of nonlinear signals and/or definitions of the SNR, an increase of the SNR has been reported by Loerincz et al (1996) and by Khovanov and Anishchenko (1997).

\subsection{Non-conventional forms of stochastic resonance}

The LRT picture led quickly to the realization that SR need not be restricted to systems of the kind that can be characterized by static bistable potentials, in which it had been discovered. One consequence was, as already mentioned, the observation of SR in monostable systems (see section 4.3.2). Another was the observation of SR in bistable systems whose attractors are coexisting limit cycles (rather than the minima of a static potential) which we now consider. As we shall see, the phenomenon can be discussed in terms of LRT although, since the system now under consideration is of the non-equilibrium type, the susceptibility cannot be obtained through the fluctuation dissipation relations (55).

4.3.1. Stochastic resonance for coexisting periodic attractors. The system considered by Dykman et al (1993b, 1994c) was the underdamped single-well Duffing oscillator (8) and (9) driven by a nearly resonant force $F \cos \omega_{F} t$ with the frequency $\omega_{F}$ close to the oscillator eigenfrequency $\omega_{0}$ such that

$$
\Gamma,|\delta \omega| \ll \omega_{F} \quad \gamma \delta \omega>0 \quad \delta \omega=\omega_{F}-\omega_{0} .
$$

It is of particular interest in view of its importance in nonlinear optics (Gibbs 1985, Drummond and Walls 1980, Flytzanis and Tang 1980, Goldstone and Garmire 1984) and its relevance to experiments on a confined relativistic electron excited by cyclotron resonant radiation (Gabrielse et al 1985, Dehmelt 1990). Provided that $F^{2} \ll \omega_{0}^{4}\left(\delta \omega^{2}+\Gamma^{2}\right) /|\gamma|$, and that the noise is weak, the resultant comparatively small amplitude $\left(\ll\left(\omega_{0}^{2} /|\gamma|\right)^{1 / 2}\right)$ oscillations of $x(t)$ can conveniently be discussed in terms of the dimensionless parameters (Dykman and Krivoglaz 1979)

$$
\eta=\Gamma /|\delta \omega| \quad \beta=\frac{3|\gamma| F^{2}}{32 \omega_{F}^{3}(|\delta \omega|)^{3}} \quad \alpha=3|\gamma| D / 8 \omega_{F}^{3} \Gamma
$$


which characterize respectively the frequency detuning, the strength of the main periodic field and the noise intensity. The bistability (Landau and Lifshitz 1976) in which we are interested arises for a restricted range of $\eta$ and $\beta$, within the triangular region bounded by the full lines of figure 20. Thus, as the amplitude of the periodic force is gradually increased from a small value at fixed frequency (see the vertical line $\mathrm{a}^{-} \mathrm{a}^{\prime}$ ), the system moves from monostability (one small-amplitude limit cycle), to bistability (two stable limit cycles of different radii) and then back again to monostability (one large-radius limit cycle). The effect of weak noise $f(t)$ is to cause small vibrations about the attractors, and to induce occasional transitions between them when the system is within the bistable regime. Since the system is far away from thermal equilibrium, the transition probabilities are not given by the classical Kramers (1940) theory. As mentioned above, the nearly resonantly driven oscillator was the first physical system without detailed balance where escape rates were explicitly calculated (Dykman and Krivoglaz 1979). The inter-attractor transitions give rise to a stationary distribution of the oscillator over the attractors. They also give rise to an SR effect which, as we shall see, occurs in the close vicinity of the kinetic phase transition (KPT) line (Dykman and Krivoglaz 1979, Dykman et al 1990e), represented by a dashed curve in figure 20 , where the stationary populations of the two attractors $w_{1}$ and $w_{2}$ are equal,

$w_{1} \approx w_{2}$

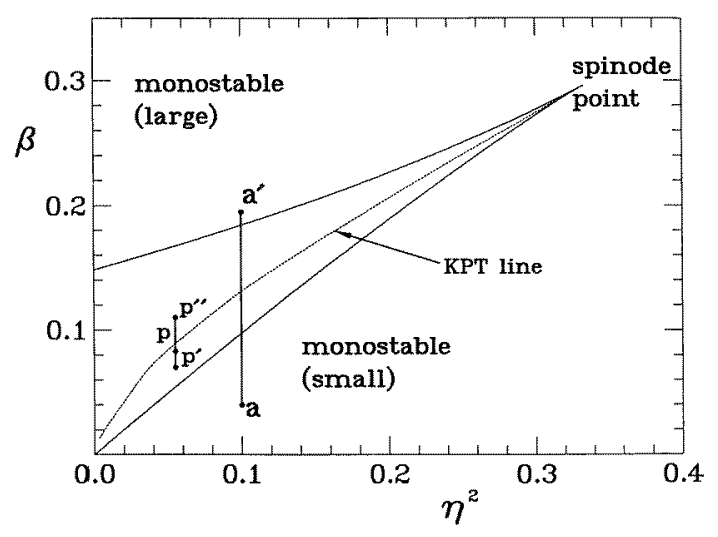

Figure 20. The phase diagram for the system (8) and (61) in terms of reduced parameters (62): the cuts $\mathrm{a}-\mathrm{a}^{\prime}$ and $\mathrm{p}^{\prime}-\mathrm{p}-\mathrm{p}^{\prime \prime}$ are discussed in the text (Dykman et al 1993b).

Our principal aim is to consider the response of the system (8) to an additional weak trial force $A \cos (\Omega t+\phi)$. In stationary conditions, the response to this field in the presence of the strong driving field can still be described, in terms of LRT, by a susceptibility. The trial force beats with the main periodic force and thus gives rise to vibrations of the system, not only at $\Omega$, but also at the combination frequencies $\left|\Omega \pm 2 n \omega_{F}\right|$ (and also at $\left|\Omega \pm(2 n+1) \omega_{F}\right|$ in the case of nonlinearity of a general type). We are interested in the case where the strong and trial forces are both nearly resonant: that is, $\omega_{F}$ and $\Omega$ both lie close to the oscillator eigenfrequency $\omega_{0}$. This is the case for which the response to the trial force is strongest. It is at its most pronounced at frequency $\Omega$ and at the nearest resonant combination, which for (8) is $\left|\Omega-2 \omega_{F}\right|$. The amplitudes of vibrations at these frequencies can be described respectively by susceptibilities $\chi(\Omega)$ and $X(\Omega)$, so that trial-force-induced modification of 
the coordinate $x$, averaged over noise, can be sought in the form

$\left.\delta\langle x(t)\rangle=A \operatorname{Re}\left\{\chi(\Omega) \exp [-\mathrm{i} \Omega t-\mathrm{i} \phi]+X(\Omega) \exp \left[\mathrm{i}\left(2 \omega_{F}-\Omega\right) t\right]-\mathrm{i} \phi\right]\right\}$.

Within the KPT range, $|\operatorname{Im} \chi(\Omega)|$ displays a high narrow peak, whose width is given by the transition rates and is therefore strongly noise dependent (Dykman and Krivoglaz 1979). The rapid rise in susceptibility with noise intensity corresponds precisely to SR because, according to (64), the areas of the peaks in the power spectrum at frequencies $\Omega$ and $\left|\Omega-2 \omega_{F}\right|$ are

$$
S(\Omega)=\frac{1}{4} A^{2}|\chi(\Omega)|^{2} \quad S\left(\left|\Omega-2 \omega_{F}\right|\right)=\frac{1}{4} A^{2}|X(\Omega)|^{2} .
$$

An intuitive understanding of the predicted mechanism of stochastic amplification (Dykman and Krivoglaz 1984) can be gained by noting that the trial force modulates the driving force (and the coordinate $x(t)$ ) at frequency $\left|\Omega-\omega_{F}\right|$ and that, when $\left|\Omega-\omega_{F}\right|$ is small compared to the dynamical relaxation rate $\Gamma$, the dynamic response of the system is almost adiabatic. In terms of the phase diagram figure 20, the beat envelope then results (Dykman et al 1993b) in a slow vertical oscillation of the operating point. If the operating point is taken to be $\mathrm{p}$, and its range of modulation $\mathrm{p}^{\prime}-\mathrm{p}^{\prime \prime}$ is set to straddle the KPT line as shown, then the role of the field $A \cos (\Omega t+\phi)$ is precisely the same as the role of the field in the case of a static potential: it alternately biases one of the stable states (a periodic attractor, in the present case) with respect to the other, i.e. it strongly modulates populations of the attractors (in the quasistatic limit). Note that this modulation does not occur away from the KPT line, where the populations are always very different.

If the noise intensity is optimally chosen, then the modulated system will have a tendency to make inter-attractor transitions coherently, once per half-cycle of the beat frequency. The net effect of the noise is, therefore, to increase the modulation depth of the beat envelope of the response, thereby increasing the components of the signal at frequencies $\Omega$ and $\left|\Omega-2 \omega_{F}\right|$.

Experimentally, the response of the system (8), (9) and (62), and the variation of the signal-to-noise ratio with $\alpha$, were investigated (Dykman et al 1993b, 1994c) by use of the electronic model described in section 2.1. In terms of scaled units the circuit parameters were set, typically, to: $2 \Gamma=0.0397, \omega_{0}=1.00, \gamma=0.1, \omega_{F}=1.07200, \Omega=1.07097$ and, to seek SR near the KPT, $F=0.068$ and the amplitude of the trial force $A=0.006$. A spectral density of fluctuations of the coordinate $x(t)$ (about $\langle x(t)\rangle$ for $A=0$ ) recorded with the above parameter values for $\alpha=0.061$ and 16384 samples in each realization, is shown in figure 21. The smooth background is the supernarrow spectral peak of Dykman et al (1990e), which is due to fluctuational transitions between the attractors and has a maximum at the field frequency $\omega_{F}$; its width is given by the transition probabilities and remains very much smaller than $2 \Gamma$. The delta function spikes, indicated by raised points of the discrete spectrum, are clearly visible, not only at the trial force frequency $(\Omega)$, but also as predicted at the mirror-reflected frequency $\left(2 \omega_{F}-\Omega\right)$. Note that the value of $\Omega$, the number of points in each $x(t)$ realization and the sample interval were chosen to be such that the signals at $\Omega$ and $\left(2 \omega_{F}-\Omega\right)$ each fell within individual bins of the discrete SDF, and such that there were several bins in between them.

The signal-to-noise ratios $R$, determined in the usual way from measurements of the delta spikes and the smooth background, are plotted (data points) as functions of noise intensity $\alpha$ in figure 22 for $\beta=0.814, \eta=0.236$. It is immediately evident that there is a range of $\alpha$ within which $R$ increases with $\alpha$. It is also apparent that, for both the main and the mirror-reflected signals, the form of $R(\alpha)$ in figure 22 is remarkably similar to that observed for conventional SR. That is, $R$ initially decreases with $\alpha$, on account of 


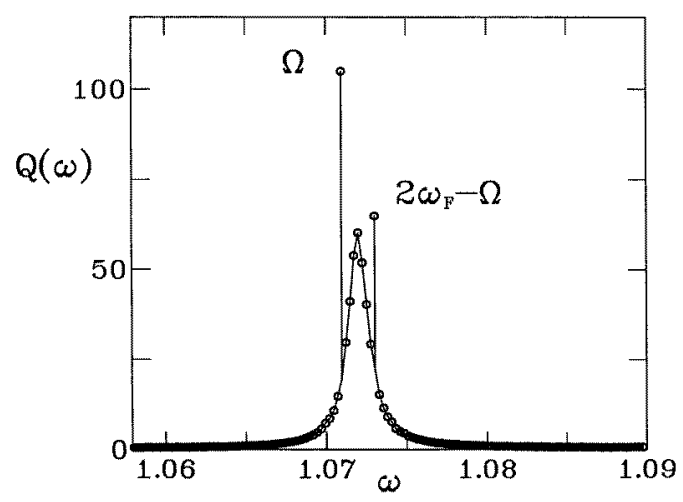

Figure 21. The power spectral density $Q(\omega)$ measured (Dykman et al 1993b) for the electronic model of (8), (9) and (61), with the contents of each FFT memory address shown as a separate data point on a highly expanded abscissa; a smooth curve, peaking at $\omega_{F}$, is drawn through the background spectrum; vertical lines indicate the delta spikes resulting from the trial force.

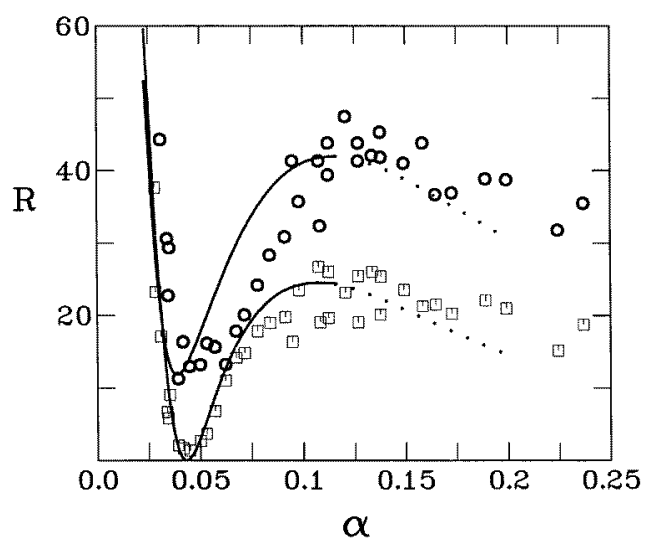

Figure 22. The signal-to-noise ratio $R$ of the response of the system (8), (9) and (61) to a weak trial force at frequency $\Omega$, as a function of noise intensity $\alpha$, in experiment and theory (Dykman et al 1993b): at the trial frequency $\Omega$ (circle data and associated theoretical curve); and at the 'mirror-reflected' frequency $\left(2 \omega_{F}-\Omega\right)$ (squares). For noise intensities near those of the maxima in $R(\alpha)$, the asymptotic theory is only qualitative and so the curves are dotted.

the increase in its denominator. With further increase of $\alpha$, the inter-attractor transitions come into play and can be effectively synchronized by the trial force, giving rise to an increase in $R$ through the stochastic amplification mechanism discussed above. Finally, for still larger $\alpha, R$ decreases again partly owing to the continuing rise in its denominator and partly because transitions are then occurring very frequently, within individual periods of the trial force, with a corresponding reduction in the proportion of the phase-coherent jumps that are responsible for the amplification.

A quantitative theory of the phenomenon is readily constructed through an extension (Dykman et al 1989, 1994c) of the formalism introduced by Dykman and Krivoglaz (1979). It leads to contributions to the susceptibilities from inter-attractor transitions of the form

$$
\chi_{\mathrm{tr}}(\Omega)=\frac{w_{1} w_{2}}{2 \omega_{F}\left(\omega_{F}-\omega_{0}\right)}\left(u_{1}^{*}-u_{2}^{*}\right) \frac{\mu_{1}-\mu_{2}}{\alpha}\left[1-\frac{\mathrm{i}\left(\Omega-\omega_{F}\right)}{W_{12}+W_{21}}\right]^{-1}
$$




$$
X_{\mathrm{tr}}(\Omega)=\frac{\underline{u}_{1}=\underline{u}_{2}}{u_{1}^{*}-u_{2}^{*}} \chi_{\mathrm{tr}}(\Omega) \quad \mu_{j}=\sqrt{\beta}\left(\frac{\partial R_{j}}{\partial \beta}\right)
$$

where $w_{1}$ and $w_{2}$ are the populations of the attractors 1 and 2 , and $W_{12}$ and $W_{21}$ are the probabilities of transitions between them, which are of the activation type $W_{i j} \propto$ $\exp \left(-R_{i} / \alpha\right) . \quad\left(u_{i}\right.$ and $u_{i}^{*}$, which define the positions of the attractors in the rotating coordinate frame, can be regarded as constants for given $\eta, \beta$; and $\mu_{i}$ determine how fast the activation energies vary with the reduced squared field amplitude $\beta \propto F^{2}$.) It is evident that the contributions (66) come into play if, and only if, the system is within the KPT range where the populations of the unperturbed attractors are comparable; otherwise, the factor $w_{1} w_{2} \propto \exp \left(-\left|R_{1}-R_{2}\right| / \alpha\right)$ will be exponentially small. Within the KPT range, however, the susceptibilities will be large because they are proportional to the large factor $\left|\mu_{1}-\mu_{2}\right| / \alpha$; the rapid increase of $W_{i j}$ with noise intensity then ensures that there will be a range of $\alpha$ in which both susceptibilities increase very rapidly with $\alpha$, consistent with the experiments. The full theory (Dykman et al 1994c) includes the effect of intra-attractor vibrations and leads to the curves of figure 22. Given the large systematic errors inherent in the measurements-arising, for example, from $\delta \omega(61)$, a small difference between large quantities which, in $\beta$ (62), is then raised to its third power-the agreement between theory and experiment can be considered excellent.

We emphasize that, in contrast to conventional bistable SR (see previously and Jung 1993, Moss 1994, Wiesenfeld and Moss 1995 and Dykman et al 1995d), stochastic amplification occurs here not at the relatively low frequency of the nearly periodic interattractor hopping but, rather, at $\Omega$ close to the much higher (tunable) frequency $\omega_{F}$ of the main periodic driving force. To emphasize the distinction, it seemed appropriate (Dykman et al 1993b, 1994c) to refer to the new form of SR as high-frequency stochastic resonance (HFSR).

There is an interesting connection between HFSR and four-wave mixing in nonlinear optics (Shen 1984). In effect, the results correspond to noise-enhanced amplification of the signal wave, and noise-enhanced generation of the idler wave. The mechanisms are resonant and, although they have the appearance of four-wave mixing, they actually correspond to multiple-wave processes: in terms of quantum optics, the oscillator absorbs and re-emits many quanta of the strong field. The very high amplification/generation coefficients arise partly from their resonant character and partly from the fact that the signal sizes correspond, not to the amplitudes of vibrations about the attractors but, as usual in bistable SR, to the 'distance' between the attractors (to their coordinate separation for conventional SR, and approximately to their difference in amplitude in the present case).

The prediction and demonstration of HFSR for periodic attractors, and its similarity (figure 22) to conventional SR, led to a broader and more general perception of the physical nature of bistable SR. Like the onset of supernarrow peaks in the power spectra, conventional SR and HFSR are both critical phenomena that arise in the range of the KPT. While HFSR is to be anticipated for coexisting stable limit cycles with equal periods, low-frequency SR is a more robust effect. It arises, not only for systems fluctuating in simple double-well potentials but also for systems where one (or both) of the attractors is chaotic (Anishchenko et al 1993, 1994); it follows from the analysis by Dykman and Krivoglaz (1979) that lowfrequency SR is also to be anticipated for periodic attractors, provided the oscillator lacks inversion symmetry, so that the centres of the coexisting periodic attractors have different locations. Since noise gives rise to fluctuational hopping between any types of attractors, SR is actually a phenomenon characteristic of all systems with coexisting attractors, regardless of the nature of those attractors, provided only that the system lies within its KPT range (63). 
4.3.2. Stochastic resonance in monostable systems. As already remarked above, the LRT perception of SR embodied in equations (52), (54) and (55) immediately implies that SR can be anticipated quite generally in systems which, within a certain frequency range, exhibit a sharp increase of the susceptibility $\chi(\omega)$ with increasing intensity $D$ of the driving Gaussian noise. The effect may be expected to be particularly strong if the response is resonant, as is the case for a noise-driven underdamped nonlinear oscillators (Dykman and Krivoglaz 1984). Because of nonlinearity, the frequency of the oscillator vibrations $\omega(E)$ depends on the energy $E$ (measured from the bottom of the potential well). Because of the noise, the oscillator has a distribution over energy (the Boltzmann distribution, in the case of systems in thermal equilibrium). Therefore, it also has a distribution over vibrational frequency. With increasing noise intensity this distribution changes and, in particular, vibrations can be excited with frequencies that were effectively unavailable for smaller noise intensity. Consequently, the response at such frequencies can be strongly enhanced by noise.

The resonant susceptibility of nonlinear oscillators has been analysed in detail theoretically, as this is one of only a few exactly solvable nonlinear problems (Dykman and Krivoglaz 1984). Explicit analytical results have been obtained for an arbitrary ratio between the relaxation rate and the noise-induced frequency straggling (Dykman and Krivoglaz 1971), and a general numerical algorithm has been formulated for calculating the shape of the power spectrum near its maximum (Dykman et al 1989). In particular, the sharp change of the power spectrum of the oscillator, including its exponential increase with increasing noise intensity, was discussed in the above papers. The appearance of an additional zerodispersion spectral peak (ZDP), in cases where the damping is extremely small and the oscillator frequency $\omega(E)$ is non-monotonic as a function of energy, was predicted and discussed by Soskin $(1989,1992)$.

The model used (Stocks et al 1992) to predict and observe SR in monostable systems and investigate its characteristic features is the tilted single-well Duffing oscillator driven by Gaussian white noise plus a weak periodic force

$$
\begin{aligned}
& \ddot{x}+2 \Gamma \dot{x}+\frac{\mathrm{d} U(x)}{\mathrm{d} x}=f(t)+A \cos \Omega t \\
& U(x)=\frac{1}{2} x^{2}+\frac{1}{4} x^{4}+B x \\
& \Gamma \ll 1 \quad\langle f(t)\rangle=0 \quad\left\langle f(t) f\left(t^{\prime}\right)\right\rangle=4 \Gamma D \delta\left(t-t^{\prime}\right) .
\end{aligned}
$$

It is effectively identical to the symmetrical single-well Duffing oscillator (8) except for the $B x$ term in the potential. We consider two distinct cases, depending on the magnitude of $|B|$.

In case (a), where $|B|<8 /(7)^{3 / 2} \approx 0.43$, the variation of the oscillator's eigenfrequency $\omega(E)$ with energy $E$ measured from the bottom of the potential well is monotonic (Dykman et al 1990b), as sketched in figure 23(a). In the absence of the periodic force $(A=0)$, for small noise intensity $D$, a narrow Lorentzian peak of width $\sim \Gamma$ occurs in the SDF at frequency $\omega(0)$. As $D$ is increased, the peak broadens asymmetrically (Dykman and Krivoglaz 1971) towards higher frequencies, as discussed earlier. For an $\Omega>\omega(0)$, initially on the tail of the peak, i.e. $(\Omega-\omega(0))>\Gamma$, the magnitude of $Q^{(0)}(\Omega)$ therefore increases extremely rapidly (Dykman and Krivoglaz 1971) with $D$, as does also the generalized susceptibility $\chi(\Omega)$. One would expect, therefore, to observe SR in this case even though the system is monostable, with $U(x)$ a smooth single-well potential. The resonance maximum is to be expected when $D$ has been 'tuned' to adjust the characteristic energy $\tilde{E} \sim D$ such that $\omega(\bar{E}) \sim \Omega$. 

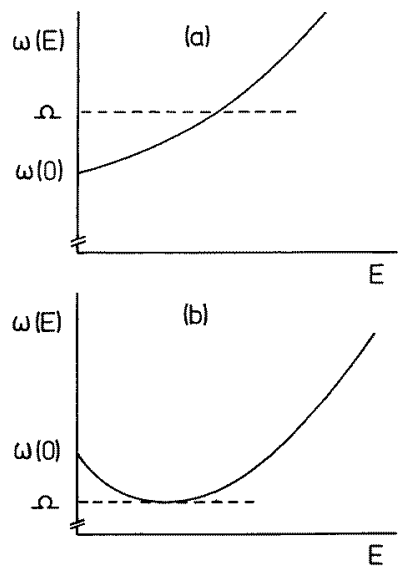

Figure 23. Sketches to show the dependence of the eigenfrequency $\omega(E)$ on energy $E$ measured from the bottom of the potential well for the system (67): (a) for $|B|<0.43$; (b) for $|B|>0.43$ (Stocks et al 1993c). Frequencies $\Omega$ at which a weak periodic force will be amplified by SR are indicated.

In case (b) with $|B|>0.43$, on the other hand, $\omega(E)$ is non-monotonic (Dykman et al 1990b), as sketched in figure 22(b). In the absence of the periodic force $(A=0)$, the system exhibits noise-induced spectral narrowing (Dykman et al 1990b); and, for sufficiently small values of the damping constant $\Gamma$, exceedingly sharp ZDPs of width $\propto \Gamma^{1 / 2}$ appear (Soskin 1989, 1992) close to the frequency $\omega_{\mathrm{m}}$ of the extremum where $\mathrm{d} \omega(E) / \mathrm{d} E=0$ (respectively, $E=E_{\mathrm{m}}$ ). A set of power spectra $Q^{(0)}(\omega)$ showing the evolution of the ZDP with increasing $D$, measured in an analogue electronic experiment (Stocks et al 1993c), is shown in figure 24 . The magnitude of the ZDP rises exponentially with increasing $D$. Just as in case (a) the corresponding rapid increase of $\chi(\Omega)$ implies a manifestation of SR for $\Omega$ close to $\omega_{\mathrm{m}}$. Since the ZDP arises only when vibrations of energy $\sim E_{\mathrm{m}}$ are excited, the intensity of the ZDP displays an activation dependence on noise intensity. This suggests that the SR effect in case (b) may be very pronounced. Case (a) is, of course, the more generic, as it applies to any underdamped system (Dykman and Krivoglaz 1984), and here too the SR effect can be quite pronounced (Dykman et al 1996a). Note, however, that systems with one or more zero-dispersion points, as in figure 22(b), are not uncommon: see section 7.2.

To test these predictions, evidence was sought (Stocks et al 1993c) of SR in an electronic model of (67); it was very similar to that shown in figure 2, except that an additional constant voltage representing the asymmetry term $B$ in (67) was added at point A. The parameter values used were $\Gamma=0.011, A=0.020$ and $B=0$ or $B=2.00$ for cases (a) or (b), respectively. The value of $\Gamma$ was again too small to be determined reliably from the circuit components (owing to the effect of stray capacitance and other non-idealities); instead, it was measured in a separate experiment. The model was driven with quasi-white noise from an external noise generator, and with a weak periodic force from an HP3325 frequency synthesizer. The resultant fluctuating $x(t)$ was digitized in 1024 point blocks and ensemble averaged by a Nicolet LAB 80 data processor to yield $\langle x(t)\rangle$. By averaging in the time (rather than the frequency) domain, as discussed in section 4.2, it was possible to make measurements of the phase shift $\phi$ between the drive and the response (Gammaitoni et al 1991a, Dykman et al 1990c, d, 1992b) as well as yielding directly the amplitude $a$ of the 

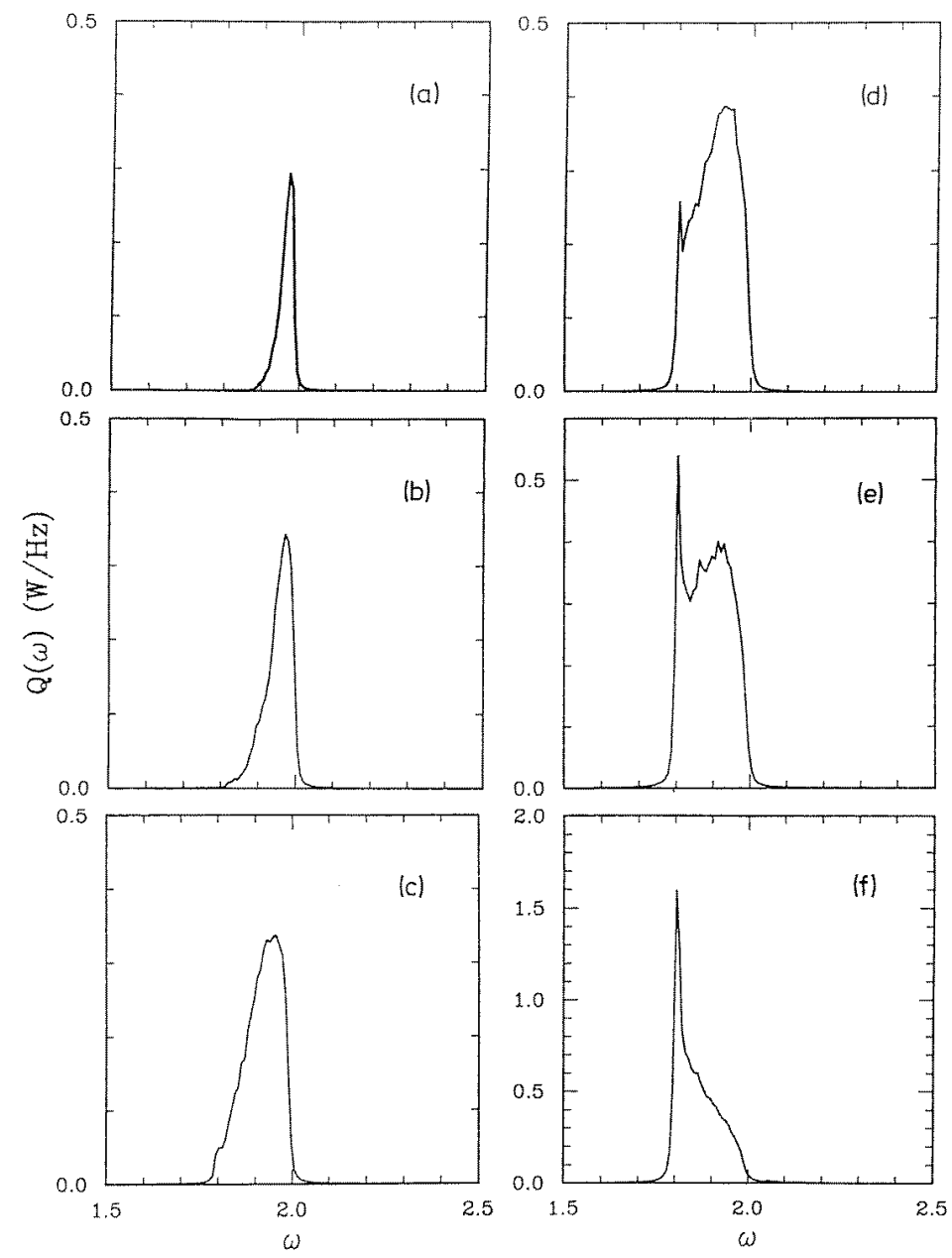

Figure 24. Spectral densities of fluctuations measured (Stocks et al 1993b) for an analogue electronic circuit model of (67) with $A=2$ and: (a) $D=0.100$; (b) 0.203 ; (c) 0.320 ; (d) 0.409 ; (e) 0.485 ; (f) 0.742 . The zero-dispersion peak is the sharp 'spike' that first appears in (d) and then grows rapidly with increasing $D$.

response. Measurements were made of the stochastic amplification factor (Jung and Hänggi 1991)

$$
S(D)=a(D) / a(0)
$$

and of the corresponding value of $\phi$ over a wide range of $D$ for the two cases (a) and (b).

Some typical measurements of the noise-induced power gain are shown by the data points in figure 25 (where $S^{2}$ is plotted rather than $S$ for more convenient comparison with earlier SR results). It is immediately evident that, both for case (a) (squares) and for case (b) (circles), $S^{2}$ at first increases rapidly with noise intensity, but then passes through a maximum and decreases again, albeit more slowly. That these data should bear a striking resemblance to those obtained for conventional SR (Jung 1993, Moss 1994, Wiesenfeld and Moss 1995, Dykman et al 1995d) is, of course, no coincidence. Note that, in the case of 


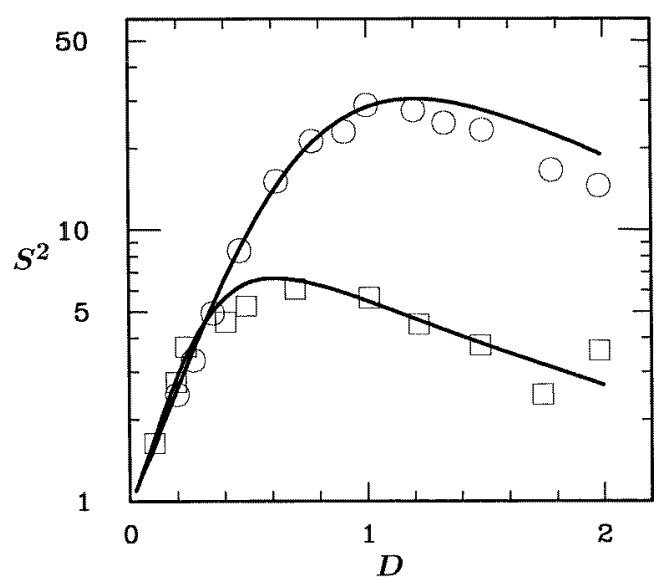

Figure 25. The squared stochastic amplification factor $S^{2}$ measured (Stocks et al 1993c) for case (a) (squares) and case (b) (circles) as a function of noise intensity $D$ for the electronic circuit model of (67) with $A=0.02$ is compared with the theoretical predictions (full curves) obtained from the fluctuation dissipation theorem.

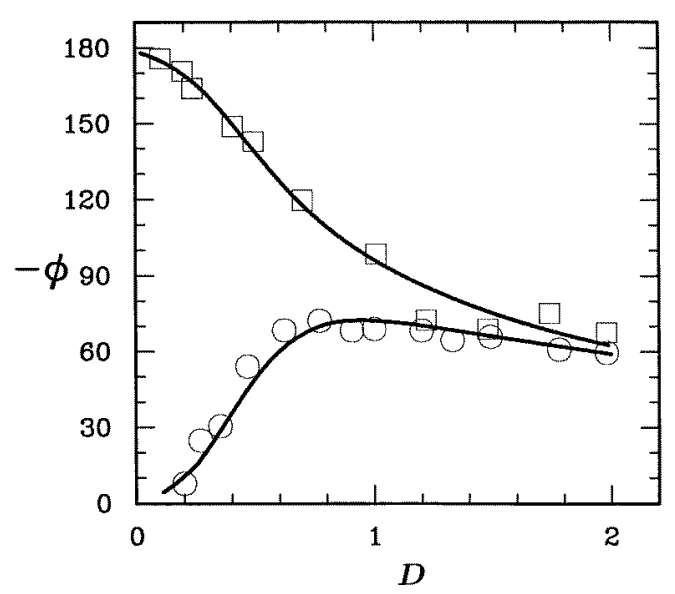

Figure 26. The phase difference $-\phi$ (in degrees) between the drive $B \cos \Omega t$ and the response $\langle x(t)\rangle$ measured (Stocks et al 1993c) for case (a) (squares) and case (b) (circles) as a function of noise intensity $D$ for the electronic circuit model of (67) with $A=0.02$ is compared with the theoretical predictions (full curves) obtained from the fluctuation dissipation theorem.

a linear system (harmonic oscillator), $S^{2}$ would not increase at all but would remain equal to unity irrespective of the value of $D$. As anticipated, the maximum is much larger for case (b) than for case (a); it can be shown (Stocks et al 1992) that, in case (b) for sufficiently small $\Gamma$, not only the signal but also the SNR increases with increasing $D$ within a certain range. We note, however, that as always in SR this increase is achieved at the expense of very poor signal transduction for small noise intensity.

The measured phase lag $-\phi$ between the drive and response is plotted for case (a) (squares) and case (b) (circles) in figure 26. The forms of $-\phi(D)$ for the two cases are strikingly different, but they can readily be understood qualitatively by analogy with a conventional (deterministic) resonance. In case (a) for $D=0$, the periodic driving 
force is being applied at a frequency well beyond the natural frequency of the system, $\Omega>\omega(0)$ (see figure 23(a)). Consequently $-\phi$ is close to $180^{\circ}$. As $D$ is increased, however, the 'natural frequency' $\omega(E)$ is effectively being tuned past the fixed driving frequency. Near resonance $-\phi$ passes through $90^{\circ}$ and, in the high $D$ limit where the 'natural frequency' substantially exceeds $\Omega,-\phi$ decreases towards $0^{\circ}$ exactly as would be seen in a conventional resonance. The phase changes for case (b), while quite different, can be accounted for in a very similar way. In this case, the 'natural frequency' $\omega(E)$ always exceeds that of the drive $\Omega$ (figure 23(b)), and so the phase lag $-\phi$ is always less than $90^{\circ}$, although it approaches $90^{\circ}$ near the resonance maximum, just as expected.

A quantitative theoretical description of these phenomena is readily developed using the LRT relations. The squared amplitude of the response is just $a^{2}=A^{2}|\chi(\Omega)|^{2}$, so that

$$
S^{2}=[a(D) / a(0)]^{2}=|\chi(\Omega)|^{2}\left\{\left[\omega(0)^{2}-\Omega^{2}\right]^{2}+4 \Gamma^{2} \Omega^{2}\right\} .
$$

The quantity $Q^{(0)}(\omega)$ in (55), the SDF in the absence of the periodic force, was obtained from equation (20) of Dykman et al (1990b) (which specifies for the model (67) the general expressions of Dykman and Krivoglaz (1984)) also using their equations (4a), 4(b), (14), (A7), (A9) and a numerical solution of their (16). Values of $S^{2}$ and $\phi$ calculated in this way for (67) with the parameters used in the circuit are plotted (full curves) as functions of $D$ in figures 25 and 26 for comparison with the experimental measurements. Given that there are no adjustable parameters, the agreement between experiment and theory can be regarded as excellent.

A closely related effect, in which a noise-induced increase occurred in the resonant response of an underdamped nonlinear oscillator, was investigated by Dykman et al (1996a) for the case of subharmonic 'two-photon' absorption. The oscillator was excited at nearly half its eigenfrequency and, here too, noise was used to tune the system to resonance, resulting in large changes in the susceptibilities. Formally, the response to the driving is nonlinear but, in the approximation where only resonant effects are taken into account, the theory can effectively be reduced to a quasilinear one. Sharp and very strong increases of the appropriate nonlinear susceptibility with increasing noise intensity were observed in the ranges where the frequency of the field $\Omega$ was close to one half of the oscillator eigenfrequency $\omega_{0}$, or to one half of the zero-dispersion frequency $\omega_{e}$.

Although the results of figure 25 show dramatic noise-induced signal enhancements they do not, even for case (b), quite satisfy the stronger definition of SR (increase of the SNR) mentioned earlier; a smaller value of $\Gamma$ would have been needed (Stocks et al 1992) for this to be so. It was pointed out by Soskin (private communication), however, that much stronger SR effects were to be anticipated in underdamped SQUIDs (superconducting quantum interference devices), even for relatively large values of $\Gamma$. His hypothesis was tested (Kaufman et al 1996) by experiments on an analogue electronic model of a SQUID.

The dynamics of the magnetic flux through a periodically-driven SQUID loop can be described in terms of a resistively shunted model (Barone and Paterno 1982) whose governing equation, after appropriate changes of variable (see, e.g., Kaufman et al 1996) can be written

$$
\begin{aligned}
& \ddot{x}+2 \Gamma \dot{x}+\frac{\mathrm{d} U}{\mathrm{~d} x}=f(t)+A \cos (\Omega t) \\
& U(x)=\frac{1}{2} B\left(x-x_{\mathrm{dc}}\right)^{2}-\cos x \\
& \langle f(t)\rangle=0 \quad\left\langle f(t) f\left(t^{\prime}\right)\right\rangle=4 \Gamma D \delta\left(t-t^{\prime}\right)
\end{aligned}
$$


corresponding to classical motion in the potential $U(x)$ under influence of the additive noise $f(t)$. We consider the case where the amplitude $A$ of the periodic force is small, where the constant $\Gamma$ is also small so that motion in the potential is underdamped and where the relative magnitudes of $B$ and $x_{\mathrm{dc}}$ are such that the potential has a single potential well.

The corresponding $\omega(E)$ dependence calculated for the SQUID potential (70) with $B=0.3$ and $x_{\mathrm{dc}}=0$, shown in figure 27, exhibits a local maximum and two local minima within the range plotted. Each of these extrema may be expected to produce a ZDP in $Q^{(0)}(\omega)$ that could in principle give rise to SR. To test this inference, Kaufman et al (1996) built an analogue electronic model of equations (70), as shown in block form in figure 28. $A^{\prime} \cos \Omega^{\prime} t^{\prime}$ and $f^{\prime}\left(t^{\prime}\right)$ are respectively a signal and an external noise applied to the underdamped nonlinear oscillator. $A^{\prime}$ is the amplitude of the signal in volts, $f^{\prime}\left(t^{\prime}\right)$ is the value of the noise voltage applied to the circuit, and $\Omega^{\prime}$ and $t^{\prime}$ are the real frequency and time in units of $\mathrm{Hz}$ and $\mathrm{s}$, respectively. Setting to zero the total currents at the inputs

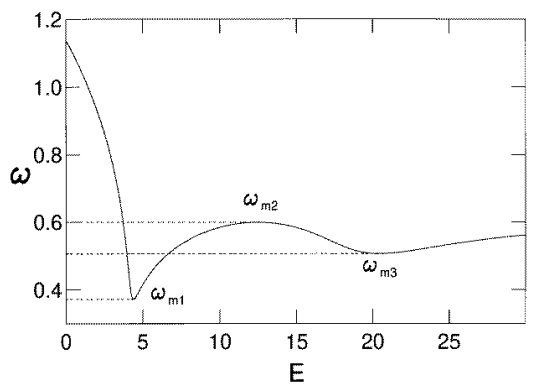

Figure 27. Calculated variation of the eigenfrequency $\omega(E)$ with energy $E$ for the potential (70) with $B=0.3, x_{\mathrm{dc}}=0$ (Kaufman et al 1997). The dashed lines indicate the positions of the first three extrema $\omega_{\mathrm{m} 1}=0.372, \omega_{\mathrm{m} 2}=0.600$ and $\omega_{\mathrm{m} 3}=0.506$.

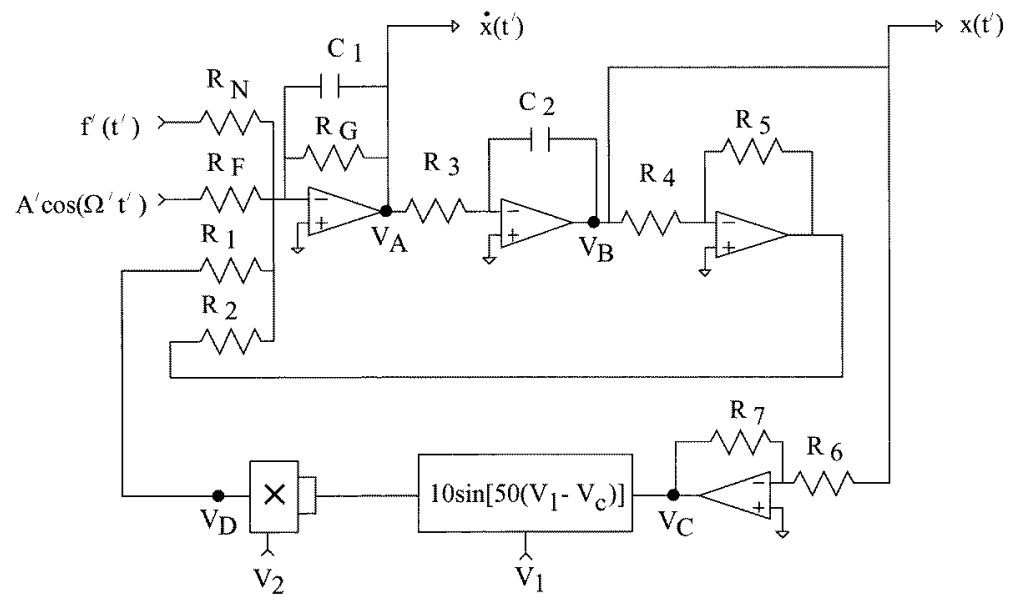

Figure 28. A block diagram of the analogue electronic circuit model of (70). Its behaviour can conveniently be analysed in terms of the voltages $V_{\mathrm{A}}, V_{\mathrm{B}}, V_{\mathrm{C}}$ and $V_{\mathrm{D}}$ at the points indicated (see text) (Kaufman et al 1997). 
of the operational amplifiers whose outputs are $V_{\mathrm{A}}$ and $V_{\mathrm{B}}$, respectively, we obtain

$$
\begin{aligned}
& \frac{V_{\mathrm{A}}}{R_{G}}+C_{1} \frac{\mathrm{d} V_{\mathrm{A}}}{\mathrm{d} t^{\prime}}+\frac{f^{\prime}\left(t^{\prime}\right)}{R_{N}}+\frac{A^{\prime} \cos \left(\Omega^{\prime} t^{\prime}\right)}{R_{F}}-\frac{\left(R_{5} / R_{4}\right) V_{\mathrm{B}}}{R_{2}}+\frac{V_{\mathrm{D}}}{R_{1}}=0 \\
& C_{2} \frac{\mathrm{d} V_{\mathrm{B}}}{\mathrm{d} t^{\prime}}+\frac{V_{\mathrm{A}}}{R_{3}}=0 .
\end{aligned}
$$

The trigonometric IC was configured to give an output of $10 \sin \left[50\left(y_{1}-y_{2}\right)\right]$, where the two inputs $y_{1}$ and $y_{2}$ are in volts and the argument of the sine is in degrees. The IC operation is restricted to lie within the range $\pm 500^{\circ}$. In order to increase the dynamic range of $x$ encompassed by the model, an analogue multiplier was used as shown to convert the argument to the double angle. The voltage at its output, in terms of the voltage $V_{\mathrm{C}}$ at the input of the trigonometric IC and the constant voltages $V_{1}$ and $V_{2}$, and allowing for internal scaling by a factor of 0.1 , is

$$
V_{\mathrm{D}}=0.1\left[10 \sin \left(50\left(V_{1}-V_{\mathrm{C}}\right)\right)\right]^{2}+V_{2}
$$

or, in terms of the double angle, now expressed in radians,

$$
V_{\mathrm{D}}=5\left(1-\cos \left[\frac{\pi}{1.8}\left(V_{1}-V_{\mathrm{C}}\right)\right]\right)+V_{2} .
$$

The voltage $V_{\mathrm{C}}$ is just

$$
V_{\mathrm{C}}=-\frac{R_{7}}{R_{6}} V_{\mathrm{B}}
$$

Eliminating $V_{\mathrm{A}}, V_{\mathrm{C}}$ and $V_{\mathrm{D}}$ from (71), (72), (74) and (75), and writing $V_{\mathrm{B}} \equiv x$, the differential equation for the voltage $x$ in the circuit can therefore be written

$$
\begin{gathered}
R_{1} C_{1} R_{3} C_{2} \frac{\mathrm{d}^{2} x}{\mathrm{~d} t^{\prime 2}}+\frac{R_{1}}{R_{G}} R_{3} C_{2} \frac{\mathrm{d} x}{\mathrm{~d} t^{\prime}}+\frac{R_{1} R_{5}}{R_{2} R_{4}} x-5\left(1-\cos \left[\frac{\pi}{1.8}\left(V_{1}+\frac{R_{7}}{R_{6}} x\right)\right]\right)-V_{2} \\
=\frac{R_{1}}{R_{F}} A^{\prime} \cos \Omega^{\prime} t^{\prime}+\frac{R_{1}}{R_{N}} f^{\prime}\left(t^{\prime}\right)
\end{gathered}
$$

where we have chosen

$$
\begin{aligned}
& R_{N}=R_{F}=100 \mathrm{k} \Omega \quad R_{1}=R_{3}=100 \mathrm{k} \Omega \\
& R_{4}=R_{5}=R_{6}=10 \mathrm{k} \Omega \quad R_{7}=11.459 \mathrm{k} \Omega \\
& R_{G}=22 \mathrm{M} \Omega \quad C_{1}=C_{2}=10 \mathrm{nF} \quad V_{1}=-0.9 \mathrm{~V} .
\end{aligned}
$$

The multi-well and single-well cases of the potential (26) correspond to different values of the parameters $R_{2}$ and $V_{2}$. For example, on introducing $R_{2}=100 \mathrm{k} \Omega, V_{2}=-3.93 \mathrm{~V}$, the time constant $\tau^{\prime}=R_{1} C_{1} / \sqrt{5}=R_{3} C_{2} / \sqrt{5}$ and the damping constant $\Gamma^{\prime}=R_{1} /\left(R_{G} \sqrt{5}\right)$, equation (32) can be reduced to

$$
\tau^{\prime 2} \ddot{x}+\Gamma^{\prime} \tau^{\prime} \dot{x}+0.2(x-1.07)+\sin (2 x)=0.2 A^{\prime} \cos \Omega^{\prime} t^{\prime}+0.2 f^{\prime}\left(t^{\prime}\right)
$$

whose parameters are readily related to those in the model (70) by means of the scaling relations

$$
\begin{array}{rlrl}
x & \rightarrow 2 x & \tau=\frac{\tau^{\prime}}{\sqrt{2}} \quad t=\frac{t^{\prime}}{\tau} \quad \Omega=\Omega^{\prime} \tau & \Gamma=\Gamma^{\prime} \sqrt{2} \\
B & =0.1 & x_{e}=2.14 \\
A & =0.2 A^{\prime} & & f(t)=0.2 \sqrt{2} f^{\prime}\left(t^{\prime}\right) .
\end{array}
$$


The nominal value of $2 \Gamma$ was 0.00144 ; the actual (see section 2.1 ) value, measured experimentally by two independent methods (McClintock et al 1993), was found to be $2 \Gamma=0.0012$ for the multi-well case and $2 \Gamma=0.0011$ for the single-well case.

When the model was driven by quasi-white noise from an external noise generator, with $A=0$, the measured spectral density $Q^{(0)}(\omega)$ underwent dramatic changes of shape with increasing $D$, as shown in figure 29. The three ZDPs appeared sequentially as $D$ 'tuned' the oscillator to different ranges of $\omega(E)$. When the weak periodic force $A \cos (\Omega t)$ was also added, with $\Omega$ chosen to lie close to the frequency of the local maximum of $\omega(E)$ and the corresponding ZDP where $|\chi|$ is expected to be strongly noise dependent, the SNR was found to vary with increasing $D$ as shown by the data points of figure 30. At first the SNR falls, as one might expect on intuitive grounds, but there follows a range of $D$ within which the SNR markedly increases with increasing $D$, i.e. a strong manifestation of SR, before falling again at very high $D$.

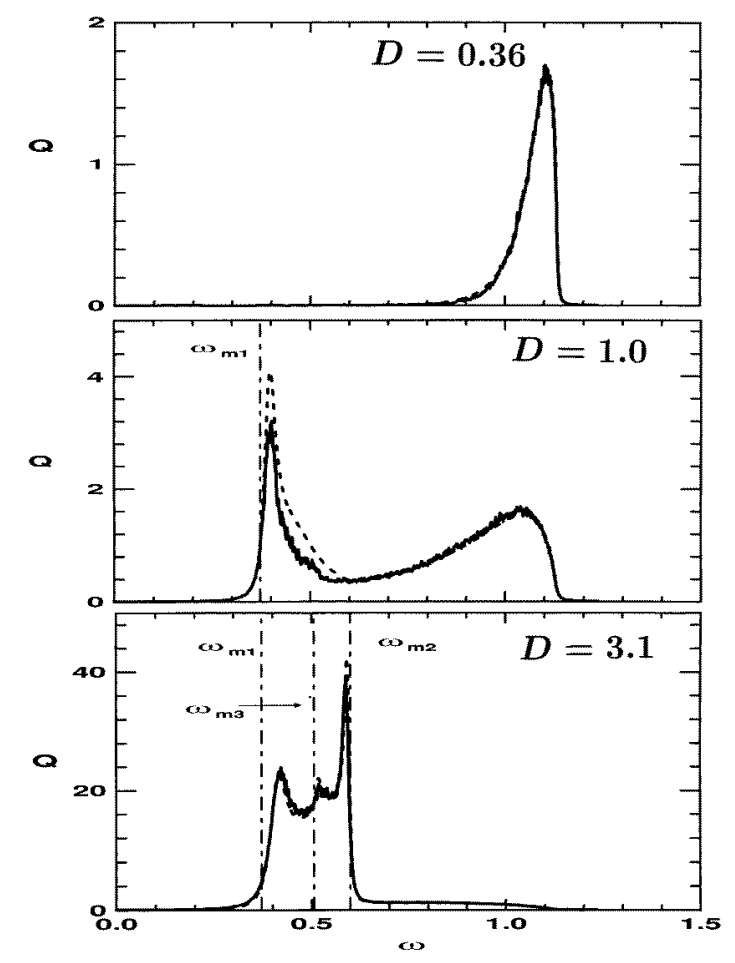

Figure 29. The spectral density of fluctuations $Q(\omega)$ measured (jagged curve) for the analogue electronic model of equations (70) with $A=0, B=0.3, x_{\mathrm{dc}}=0$, compared with the calculated behaviour (smooth curves), for three noise intensities $D$ (Kaufman et al 1997). One ZDP is seen for $D=1.0$, and three for $D=3.1$. Note the differing ordinate scales.

The theory of these phenomena has been developed (Kaufman et al 1996, 1997) on the usual LRT basis: first, the power spectral densities $Q^{(0)}(\omega)$ in the absence of the periodic force were calculated for different values of $D$, by means of an algorithm similar to that described previously (Dykman et al 1989, 1990b); second, the calculated $Q^{(0)}(\omega)$ was inserted in equations (54) and (55) to yield the SNR, as required. The theory, shown by the full curves of figures 29 and 30, is in satisfactory agreement with the measurements. It demonstrates that the size of the increase in SNR is related to the magnitude of $\Gamma$ and 


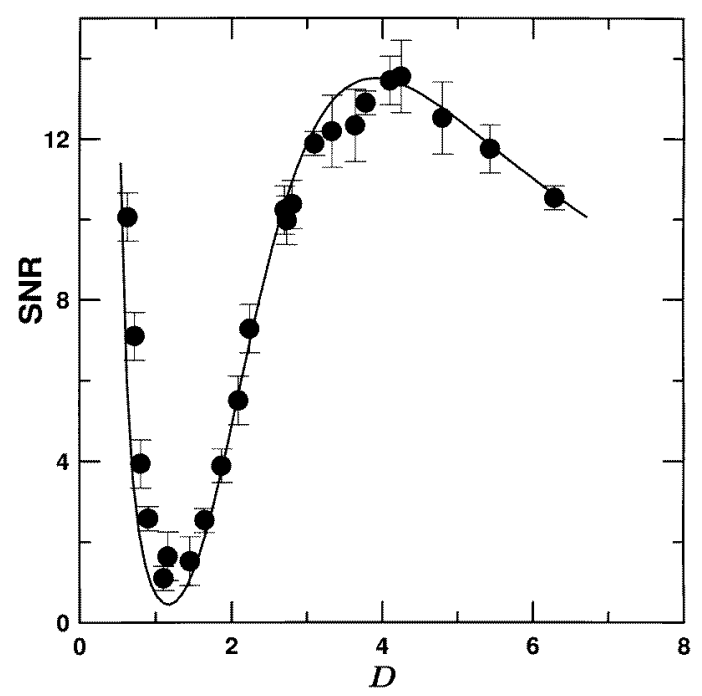

Figure 30. The signal-to-noise ratio (SNR) measured (data points) as a function of noise intensity $D$ for the analogue electronic model of equations (70) with $A=0.016, \Omega=0.62, B=0.3$, $x_{\mathrm{dc}}=0$, compared with the behaviour predicted (full curve) by LRT, equations (54) and (55), using the calculated spectral densities $Q(\omega)$ of which three examples are plotted as dashed lines in figure 29 (Kaufman et al 1997).

that, if $\Gamma$ is made small enough, there is in principle no limit to the rise in SNR that can be achieved. Note also that the above SQUID parameters, used by Kaufman et al (1996), were chosen so as to provide a monostable potential mainly in order to emphasize the marked difference between zero-dispersion SR and conventional SR; but zero-dispersion SR also occurs in SQUIDs with multi-well potentials (Kaufman et al 1997) where it exhibits some interesting features.

In the light of these results from the analogue electronic model, we may conclude that zero-dispersion SR is to be anticipated in underdamped SQUIDs. The optimal frequencies for the SR, i.e. the ZDP frequencies, are tunable over a very wide range-in principle, running from near zero up to a maximum value not much less than the Josephson plasma frequency_by adjustment of the applied static magnetic field and/or the inductance of the loop. Although the SNR at the output of an SR (or any other) device cannot exceed that at the input (see section 4.2), noise can still be used to improve the output SNR of signals for a specific nonlinear device, as in the present case. The performance of a high-frequency SR device based on an underdamped SQUID would probably be comparable with that of low-frequency SQUID-SR devices (Hibbs et al 1995), and it would have the additional advantage of being tunable over a wide range of frequencies and SQUID parameters.

4.3.3. Stochastic resonance for periodically modulated noise intensity. We now discuss SR phenomena that can occur (Dykman et al 1992a) in a bistable system when the noise and the periodic force are introduced multiplicatively, so that the former is modulated by the latter. Periodically modulated noise is not uncommon and arises, for example, at the output of any amplifier (e.g. in optics or radio astronomy) whose amplification factor varies periodically with time. It is of obvious importance, therefore, to establish whether or not a modulated zero-mean noise can give rise to a periodic signal in the system it is driving. 
Such an effect would not, of course, occur in a linear system where the signal is directly proportional to the driving noise so that they must both, on average, vanish. We shall see that in a nonlinear system, however, a periodic signal does arise and, furthermore, that in a bistable system a form of SR can occur for periodically modulated noise intensity. It has some novel features that are strikingly different from those in conventional SR.

Dykman et al (1992a) considered an overdamped Brownian particle moving in an asymmetric bistable potential

$$
\begin{aligned}
& \dot{x}+U^{\prime}(x)=f(t) \equiv\left(\frac{1}{2} A \cos (\Omega t)+1\right) \xi(t) \\
& U(x)=-\frac{1}{2} x^{2}+\frac{1}{4} x^{4}+\lambda x .
\end{aligned}
$$

Here, $\lambda$ characterizes the asymmetry of the potential. For $-2 /(3 \sqrt{3})<\lambda<2 /(3 \sqrt{3})$ the potential $U(x)$ has two minima, i.e. the system is bistable. The function $\xi(t)$ represents white Gaussian noise of intensity $D$, so that

$$
\left\langle f(t) f\left(t^{\prime}\right)\right\rangle=2 D \delta\left(t-t^{\prime}\right)\left[1+A \cos (\Omega t)+\frac{A^{2}}{8}(1+\cos (2 \Omega t))\right]
$$

i.e. the intensity of the driving force $f(t)$ is periodic in time. In what follows, we assume the modulation to be weak, $A \ll 1$, and neglect the term proportional to $A^{2}$ in (79).

For sufficiently weak noise, when $D$ is much less than the depths $\Delta U_{1,2}$ of the potential wells,

$$
\begin{aligned}
& D \ll \Delta U_{1}, \Delta U_{2} \quad \Delta U_{n}=U\left(x_{\mathrm{s}}\right)-U\left(x_{n}\right) \quad n=1,2 \\
& U^{\prime}\left(x_{1,2}\right)=U^{\prime}\left(x_{\mathrm{s}}\right)=0 \quad x_{1}<x_{\mathrm{s}}<x_{2}
\end{aligned}
$$

the motion of the system consists mostly of small intra-well fluctuations about the equilibrium positions $x_{1,2}$. Occasionally, there will be large fluctuations, sufficient to cause inter-well transitions. Periodic modulation of the noise influences both types of fluctuation, and so there are two contributions to the signal $\langle x(t)\rangle$ : one from the modulation of the intra-well fluctuations; and the other from the modulation of the populations $w_{1,2}(t)$ of the wells 1,2

$$
\langle x(t)\rangle \simeq \sum_{n=1,2}\langle x(t)\rangle_{n} w_{n}(t)
$$

where \langle\rangle$_{n}$ implies averaging over the $n$th well. In the spirit of LRT, we assume that the periodic response to the modulation can be described by a generalized susceptibility $\kappa(\Omega)$

$$
\langle x(t)\rangle=\langle x\rangle^{(0)}+A \operatorname{Re}[\kappa(\Omega) \exp (-\mathrm{i} \Omega t)]
$$

where the superscript (0) means that the corresponding quantity refers to the case $A=0$.

We consider the response for the physically important case of low-frequency modulation, $\Omega \ll U^{\prime \prime}\left(x_{1,2}\right)$, where the adiabatic approximation holds. Both the intra-well fluctuations and the transition probabilities $W_{12}$ and $W_{21}$ are then the same as they would be for white noise of instantaneous intensity $D(1+A \cos \Omega t)$. The well populations $w_{1}$ and $w_{2}$ for periodically modulated noise depend on the relationship between $\Omega$ and the $W_{n m}$.

To lowest order in the modulation amplitude $A$, the probability $W_{n m}$ of an $n \rightarrow m$ transition is

$$
W_{n m} \equiv W_{n m}(t) \simeq W_{n m}^{(0)}\left(1+A \frac{\Delta U_{n}}{D} \cos \Omega t\right)
$$

where $W_{n m}^{(0)} \propto \exp \left(-\Delta U_{n} / D\right)$ is the usual Kramers transition rate. The corresponding periodic modulation of the well populations $w_{1,2}$ is described by the balance equation 
$\dot{w}_{1}=-W_{12} w_{1}+W_{21} w_{2}$. The periodic redistribution over the wells gives a contribution $\kappa_{\text {tr }}(\Omega)$ to the susceptibility $\kappa(\Omega)$ of the form

$$
\begin{aligned}
& \kappa_{\mathrm{tr}}(\Omega)=-\frac{1}{D}\left(x_{1}-x_{2}\right)\left(\Delta U_{1}-\Delta U_{2}\right) w_{1}^{(0)} w_{2}^{(0)} \frac{W^{(0)}}{W^{(0)}-\mathrm{i} \Omega} \\
& W^{(0)}=W_{12}^{(0)}+W_{21}^{(0)} \\
& w_{1}^{(0)}=W_{21}^{(0)} / W^{(0)} \quad w_{2}^{(0)}=1-w_{1}^{(0)} .
\end{aligned}
$$

In obtaining (84) from (81)-(83), we have neglected the deviations of $\left\langle x_{n}\right\rangle$ from $x_{n}$ in comparison with $\left|x_{2}-x_{1}\right|$. According to (83) and (84),

$$
\left|\kappa_{\text {tr }}(\Omega)\right| \propto \zeta \exp (-\zeta) \quad \zeta=\left|\Delta U_{1}-\Delta U_{2}\right| / D
$$

i.e. the inter-well transitions contribute to $\kappa(\Omega)$ provided that the potential is asymmetric. This is easily understood qualitatively. For a symmetric potential, the wells are equally populated irrespective of noise intensity and so the modulation of the latter does not influence the populations $w_{1}$ and $w_{2}$. For asymmetric potentials, on the other hand, the ratio of the populations $w_{1}^{(0)} / w_{2}^{(0)} \propto \exp \left[\left(\Delta U_{2}-\Delta U_{1}\right) / D\right]$ depends sharply on the noise intensity, and will be strongly influenced by the modulation of $D$. It is also evident that, for very large $\zeta$, a weak modulation will not result in a substantial redistribution over the wells because the product $w_{1} w_{2} \propto \exp (-\zeta)$ will remain exponentially small: $\left|\kappa_{\mathrm{tr}}(\Omega)\right|$ must, therefore, vary non-monotonically with $\zeta \propto D^{-1}$, with a maximum at $\zeta=1$, and increase rapidly with $D$ in the range $\exp (\zeta) \gg 1$. This increase can in itself give rise to stochastic resonance, since the periodic signal is rising rapidly with increasing noise intensity.

However, the intra-well fluctuations are also to be considered. Their contribution to the susceptibility $\kappa(\Omega)$ is connected with the local asymmetry of the potential about each of its minima, just as for the zero-frequency peaks in the power spectra of single-well underdamped systems (Dykman et al 1991a). The partial susceptibility for the $n$th well, $\kappa_{n}(\Omega)$, can be obtained for small $D$ by expanding $U^{\prime}(x)$ in (78) to second order in $\left(x-x_{n}\right)$ and calculating $\left(x-x_{n}\right)$ formally to second order in $f(t)$. For $\Omega \ll U^{\prime \prime}\left(x_{n}\right)$ one arrives at the expression

$$
\kappa_{n}(\Omega)=-U^{\prime \prime \prime}\left(x_{n}\right)\left[U^{\prime \prime}\left(x_{n}\right)\right]^{-2} D / 2 .
$$

The susceptibility $\kappa(\Omega)$ as a whole is then given by the sum of the above contributions

$$
\kappa(\Omega)=\sum_{n=1,2} \kappa_{n}(\Omega) w_{n}^{(0)}+\kappa_{\mathrm{tr}}(\Omega)
$$

which is a general form of the susceptibility of a bistable system for low noise intensities (Dykman et al 1989).

Equations (82), (84), (86) and (87) describe completely the periodic response of the system to periodically modulated noise. As usual, we characterize the influence of the noise intensity on the response by the signal-to-noise ratio $R$ equal to the ratio of the $\delta$-like spike in the power spectral density of the fluctuations of the system (53) giving rise to the SNR $R$ (54). Note that in contrast with conventional bistable SR (section 4.2), and SR for monostable systems (section 4.3.2), the effective susceptibility $\kappa(\Omega)$ is not now given directly by the fluctuation dissipation theorem in terms of $Q^{(0)}(\omega)$.

The most interesting and important situation arises when the main contributions to both $\kappa(\Omega)$ and $Q^{(0)}(\Omega)$ are due to fluctuational inter-well transitions. In this case, (54) simplifies and, allowing for the explicit form (Dykman et al 1990c, d) of $Q^{(0)}(\Omega)$, one can (Dykman et al 1992a) obtain

$$
R \simeq R_{\mathrm{tr}}=\frac{\pi}{4} A^{2} \zeta^{2} W_{12}^{(0)} W_{21}^{(0)} /\left(W_{12}^{(0)}+W_{21}^{(0)}\right) .
$$


It can be seen from (83) and (85) that $R_{\text {tr }} \propto \zeta^{2} \exp (-\Delta U / D)$, where $\Delta U=$ $\max \left(\Delta U_{1}, \Delta U_{2}\right)$ is the depth of the deeper potential well. For non-equal well depths, it is obvious that $R_{\mathrm{tr}}$ increases sharply with increasing $D$, i.e. stochastic resonance occurs. We emphasize that (88) holds for $\zeta$ not too large: this is because the contributions to $\kappa(\Omega)$ and $Q^{(0)}(\Omega)$ from the inter-well transitions are proportional to $\exp (-\zeta)$ compared to the intra-well contribution (cf equations (84)-(87)) and, for large $\zeta$, they become small.

The theory was tested (Dykman et al 1992a) by means of an analogue experiment modelling (78). The circuit was very similar in design to that shown in figure 5(a), except for: (i) provision of a constant voltage at point A to produce the asymmetry term $A x$; and (ii) application of the scaled periodic force and the noise via an additional analogue multiplier to provide the necessary modulation, creating the $f(t)$ term. Measurements of the signal-to-noise ratio $R$ are shown by the square data points in figure 31 . We note immediately the occurrence of SR: the rate of increase of $R$ is faster than $D$, so that it does not represent merely the proportionality of the modulation to $D$ in (79). The lower full curve in figure 31 represents a fit of the theory (54), (86) and (87) to the experimental data, allowing for the explicit form (Dykman et al 1992b) of $Q^{(0)}(\Omega)$, demonstrating the universal character of the shape of the SR.

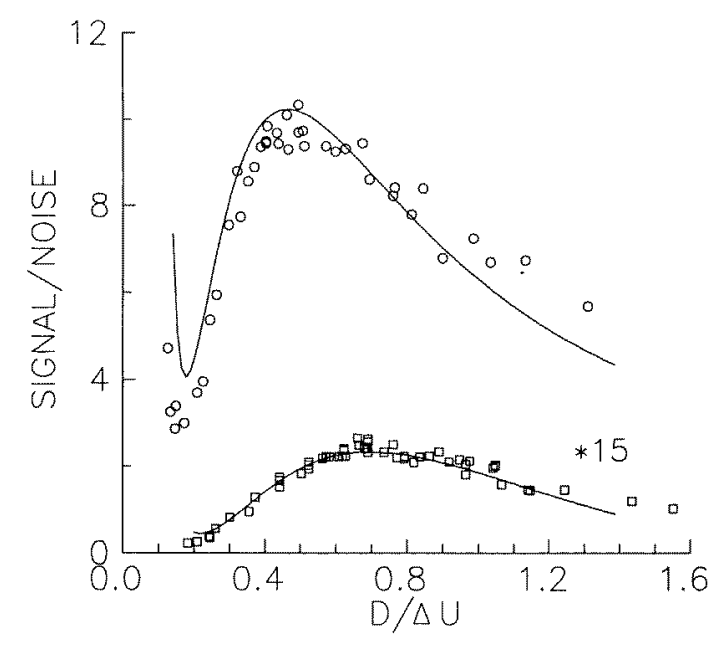

Figure 31. Measurements (square data points) of the signal-to-noise ratio $R(\times 15)$ for periodically modulated noise compared with theory (curves) as a function of reduced noise intensity $D / \Delta U$ with $A=0.14, \lambda=0.2, \Omega=0.029$ (Dykman et al 1992a). The circle data represent measurements on the same circuit with additive periodic forcing (conventional SR) under similar conditions compared with the theory (upper curves).

It is interesting to compare SR for periodically modulated driving noise with conventional SR (circle data and upper curve in figure 31) in periodically driven systems where $f(t)$ in (78) is replaced by

$$
\tilde{f}(t)=\xi(t)+A \cos \Omega t .
$$

The most substantial difference is that, in the present case, SR occurs for an asymmetric bistable potential with wells of different depths (see (85); (88)), whereas conventional SR can be regarded (Dykman and Krivoglaz 1979, 1984, Dykman et al 1990c, d) as a kinetic phase transition phenomenon that is at its most pronounced for equally populated stable states, i.e. for equal well depths. The asymmetry of the model (78) is controlled by $\lambda$, 


$$
\zeta \equiv\left|\Delta U_{1}-\Delta U_{2}\right| / D \simeq 2|\lambda| / D
$$

for $|\lambda| \ll 1$, and $R$ would, therefore, be expected (88) to increase rapidly with $\lambda$, whereas, for additive periodic forcing, $R$ decreases rapidly (Dykman et al 1990c, d) with increasing $|\lambda|$. These ideas were confirmed directly by the experimental data of figure 32(a). According to (88) and (90), $R \propto \lambda^{2}$ for small $|\lambda|$; but for large $|\lambda|$ the increase saturates because the depth of the deeper well increases, with a corresponding decrease in the contribution to $R$ from inter-well transitions. We note that, for periodic forcing of the system described by (78) and (89), $R$ should be larger than for periodic modulation of the noise for the same dimensionless amplitude $A$, just because of the additional asymmetry factor $\zeta^{2}$ in (88). It can be seen from figure 31 that the theory (full curves) is in good agreement with the experiment. The results of figure 32(b) demonstrate that the signal-to-noise ratio saturates with increasing amplitude of the periodic modulation. The effect is more striking
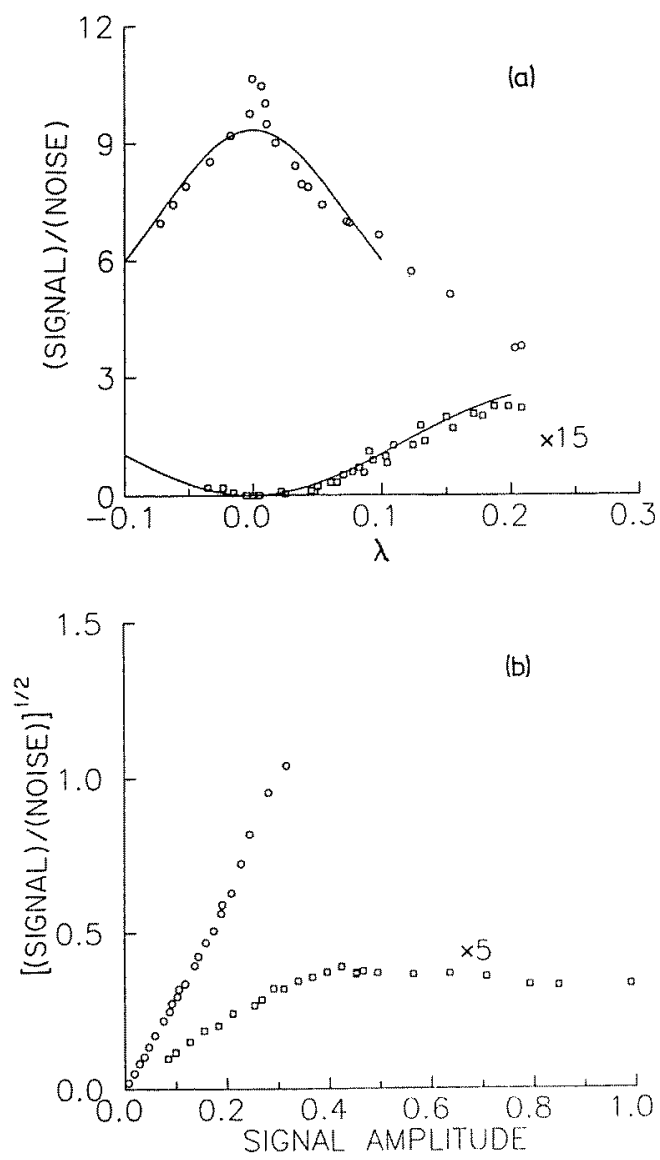

Figure 32. Measurements of the signal-to-noise ratio for periodically modulated noise (square data points) and for additive periodic forcing, i.e. conventional SR (circle data) under similar conditions, for $A=0.15, D \Delta U=0.303$ and $\Omega=0.029$. (a) The measurements are plotted directly as a function of the asymmetry parameter $\lambda$ for comparison with theory (curves). (b) (signal/noise) $)^{1 / 2}$ is plotted as a function of signal amplitude $A$ at the input, for the same $D$ and $\Omega$, with $\lambda=0.2$ : note the linear dependence seen for small signals and the saturation for strong signals, in the case of periodically modulated noise (Dykman et al 1992a). 
than the corresponding saturation in conventional SR, for which the additive periodic force distorts the shape of the potential; cf Dykman et al (1993c) where nonlinear effects for large-amplitude modulation in conventional SR are considered.

It is also interesting to note that the anisotropy of the potential, which gives rise to SR for periodically modulated noise, can also give rise to SR when the periodic modulation is parametric with the force proportional to the coordinate. This type of SR has been reported for a parametrically-driven magnetoelastic ribbon (Spano et al 1992); it would also be anticipated in parametrically-driven bistable electronic systems (Debnath et al 1989) if the potential were made asymmetrical.

In concluding this subsection we would comment that, just as in the case of conventional SR, the main features of SR with periodically modulated noise can be well described within the scope of LRT. The marked differences predicted to exist between these two types of $\mathrm{SR}$, and in particular their quite different characteristic variations of $R$ with the asymmetry of the potential, including the rapid decrease of $R$ for additive periodic forcing, have been convincingly confirmed by the analogue electronic experiments.

\subsection{Noise-enhanced heterodyning}

In the well known phenomenon of heterodyning, two high-frequency fields-an 'input signal' and a 'reference signal' - are mixed nonlinearly to generate a heterodyne signal at the difference frequency. Mixing of this kind occurs quite generally in any nonlinear system. The addition of noise usually results in a decrease in the amplitude of the heterodyne signal (and its SNR), because the frequency response of the system becomes correspondingly broadened. Nonetheless, it has been shown theoretically and experimentally (Dykman et al 1994a) that, in bistable systems of the kind that exhibit SR (see section 4.2), the heterodyne signal (and SNR) can sometimes be enhanced by an increase in the noise intensity. We now discuss how this comes about. We consider an overdamped bistable system driven by three time-dependent forces representing respectively the reference and input signals, and noise:

$$
\frac{\mathrm{d} x}{\mathrm{~d} t}=-U^{\prime}(x)+A_{\text {ref }} x \cos \omega_{0} t+A_{\text {in }}(t) \cos \left(\omega_{0} t+\phi(t)\right)+f(t) .
$$

Here, the terms proportional to $A_{\text {ref }}$ and proportional to $A_{\text {in }}(t)$ are respectively the highfrequency reference signal corresponding to a local oscillator of frequency $\omega_{0}$ (applied multiplicatively), and the modulated high-frequency input signal (applied additively). The functions $A_{\text {in }}(t)$ and $\phi(t)$ vary slowly compared to $\cos \omega_{0} t$, and it is their variation in time that has to be revealed via heterodyning. The heterodyning can be characterized by the low-frequency signal at the output, $x^{(\mathrm{sl})}(t)=\overline{x(t)}$ (the overbar stands for averaging over the period $\left.2 \pi / \omega_{0}\right)$, for $A_{\text {in }}=$ constant and $\phi=\Omega t+$ constant, with $\Omega \ll \omega_{0}$, i.e. for a monochromatic input signal whose frequency $\omega_{0}+\Omega$ is slightly different from the frequency $\omega_{0}$.

Dykman et al (1994a) considered the case where the double-well potential $U(x)$ has equally deep wells, as in standard stochastic resonance, and for convenience chose it to be the quartic potential (30). Its minima occur at $x_{n}=(-1)^{n}, n=1,2$, and the characteristic (dimensionless) relaxation time of the system $t_{\mathrm{r}} \equiv 1 / U^{\prime \prime}\left(x_{n}\right)=1 / 2$. The analysis is not, of course, limited to the particular form of equations (30) and (91) and, in fact, the effect occurs in both overdamped and underdamped systems. The effect has also been observed in a totally different class of system - a bistable all-optical cavity (Dykman et al 1995a). However, the explicit expressions take on a simpler form for the model (30) and (91); they are further simplified in the case when the frequencies of the input and reference signals 
are high compared with the reciprocal relaxation time of the system,

$$
\omega_{0} \gg t_{\mathrm{r}}^{-1} \quad\left(t_{\mathrm{r}}=\frac{1}{2}\right) .
$$

The term $f(t)$ in (91) is a random force. It is assumed to be a zero-mean Gaussian noise. Dykman et al (1994a) allowed for a form of this noise with two independent components, at low and high frequencies respectively, with the latter being randomly modulated vibrations at frequency $\omega_{0}$ :

$$
f(t)=f_{\mathrm{lf}}(t)+f_{\mathrm{hf}}(t) \quad f_{\mathrm{hf}}(t)=\operatorname{Re}\left(\tilde{\mathrm{h}}_{\mathrm{hf}}(t) \exp \left(-\mathrm{i} \omega_{0} t\right)\right) .
$$

The power spectrum $\Phi_{\mathrm{lf}}(\omega)$ of the low-frequency noise $f_{\text {lf }}(t)$ is assumed to be flat up to $\omega \sim \omega_{\mathrm{c}} \gg t_{\mathrm{r}}^{-1}\left(\omega_{\mathrm{c}}\right.$ may be small compared to $\left.\omega_{0}\right)$. The power spectrum of $f_{\mathrm{hf}}(t)$ is assumed to be centred at $\omega_{0}$ and also flat over a range greatly exceeding $t_{\mathrm{r}}^{-1}$.

For $\omega_{0} \gg t_{\mathrm{r}}^{-1}$ the motion of the system consists of fast oscillations at frequency $\omega_{0}$ (and its overtones) superimposed on a slow motion. To first order in $\omega_{0}^{-1}$, the equation for the slow part of the coordinate takes the form

$$
\begin{aligned}
& x^{(0)} \approx x^{(\mathrm{sl})} \\
& \dot{x}^{(\mathrm{sl})}=-U^{\prime}\left(x^{(\mathrm{sl})}\right)+A(t) \sin \phi(t)+f^{(0)}(t) \quad A(t)=\frac{A_{\text {ref }}}{2 \omega_{0}} A_{\text {in }}(t)
\end{aligned}
$$

where

$$
\begin{aligned}
& f^{(0)}(t)=f_{\mathrm{lf}}(t)-\frac{A_{\mathrm{ref}}}{2 \omega_{0}} \operatorname{Im} \tilde{f}_{\mathrm{hf}}(t) \quad \Phi^{(0)}(\omega) \approx D / \pi \quad \text { for } \omega \lesssim \omega_{\mathrm{c}}(95) \\
& D=D_{\mathrm{lf}}+\left(A_{\mathrm{ref}}^{2} / 2 \omega_{0}^{2}\right) D_{\mathrm{hf}} .
\end{aligned}
$$

These equations are of a familiar form. For the case where $A(t)$ and $\phi(t)$ vary slowly over the time $t_{\mathrm{r}}$, the dynamics of the system (30), (94) and (95) have already been investigated in great detail in the context of SR and, as discussed in section 4.2, the system can be well described in terms of LRT provided that $A$ is small enough. In the particular case of a periodic input signal, $A_{\text {in }}=$ constant, $\phi=\Omega t+$ constant, the average (slow) part of the coordinate $\left\langle x^{(\mathrm{sl})}(t)\right\rangle$ varies periodically at the low modulation frequency $\Omega$, and the power spectrum of the coordinate contains $\delta$-spikes at the frequencies $n \Omega$ on top of the broad spectrum. For small $A \propto A_{\text {in }}$ the vibrations of $\left\langle x^{(\mathrm{sl})}(t)\right\rangle$ are practically monochromatic, $\left\langle x^{(\mathrm{sl})}(t)\right\rangle=a_{0}+a \sin \psi(t)$, with their amplitude $a$ and phase $\psi$ given by the expression

$$
a \sin \psi(t)=-A \operatorname{Im}\left(\chi(\Omega) \mathrm{e}^{-\mathrm{i} \phi(t)}\right) \quad \phi(t)=\Omega t+\text { constant. }
$$

For small noise intensities $(\Delta U \gg D)$ in the case of a symmetric double-well potential $U(x)$, the susceptibility $\chi(\Omega)$ is (Dykman et al 1994a) of the form

$$
\chi(\Omega)=\frac{1}{t_{\mathrm{r}}^{-1}-\mathrm{i} \Omega}+\frac{W}{4 D} \frac{\left(x_{2}-x_{1}\right)^{2}}{W-\mathrm{i} \Omega} .
$$

Here, $W \equiv W_{12}+W_{21} \propto \exp (-\Delta U / D)$ is the relaxation rate of the populations of the stable states. It is seen from (96) and (97) that, since $W$ increases sharply with the noise intensity $D$, the amplitude of the heterodyne signal at the difference frequency $\Omega$ increases with noise intensity in a certain range of $D$, too. Just as in standard SR, the noise-enhanced heterodyning (NEH) can be characterized by the SNR $R$ which can be shown to be given by

$$
\begin{aligned}
& R=\pi \frac{A^{2}\left(x_{2}-x_{1}\right)^{2}}{16 D^{2}} \frac{W^{2}+\Omega^{2} t_{\mathrm{r}}^{2} \tilde{D}^{2}}{W+\Omega^{2} t_{\mathrm{r}}^{2} \tilde{D}} \quad \tilde{D}=4 D\left(x_{2}-x_{1}\right)^{-2} \\
& \Omega, \tilde{D} \ll t_{\mathrm{r}}^{-1} \quad W \ll \tilde{D}
\end{aligned}
$$


to lowest order in the small parameters in (98), cf (59) (where $\Omega_{\mathrm{r}}$ is the reciprocal of $t_{\mathrm{r}}$ in (98) and it has been set that $x_{2}-x_{1}=2$ ). It follows from (98) (and also from the more general expression for the SNR) that, in the range of noise intensities where

$$
\Omega \tilde{D} t_{\mathrm{r}} \lesssim W \lesssim t_{\mathrm{r}}^{-1}
$$

the SNR increases with increasing noise intensity. The increase is quite sharp, being nearly exponential. This means that noise-enhanced heterodyning would be expected to arise in a bistable system, whether driven by a low- or a high-frequency noise (or both).

An intuitive picture of the physical mechanism underlying NEH is as follows. The reference force provides a modulation of the potential $(-1 / 2) A_{\text {ref }} x^{2} \cos \omega_{0} t$ which is even in the coordinate, and therefore does not break the symmetry of the system. The phases of the vibrations $\delta x_{\text {ref }}(t)$ about the minima of the wells caused by this signal differ by $\pi$ (i.e. the vibrations are in counterphase). The situation is quite different for the input signal $A_{\text {in }}$. This signal, being additive, breaks the symmetry of the potential, and the amplitude and phase of the periodic vibrations of the coordinate $\delta x_{\text {in }}(t)$ at the signal frequency $\omega_{\text {in }}$ are the same in the absence of the reference signal for both wells (the vibrations are in phase). When both forces are present, they combine nonlinearly giving rise to potential terms oscillating at combination frequencies, and in particular to a term at the difference frequency $\left|\omega_{0}-\omega_{\text {in }}\right|$ which is proportional, approximately, to the product of $\delta x_{\text {ref }} \times \delta x_{\text {in }}$. This term defines the slow motion of the system. Obviously, it is antisymmetric: its sign is opposite for the two wells.

The amplitude of the fast oscillations induced in the system by the superimposed reference and input signals varies slowly in time, as the effects of the individual signals tend alternately to reinforce or cancel each other. These amplitude variations occur in antiphase between the two wells: when the amplitude of the fast oscillation is relatively large in one well, it is relatively small in the other, and vice versa. The particular well for which the fast vibrations are of the larger amplitude at a given time will also be the one from which fluctuational transitions are most likely to occur. This is because the centre of oscillations is then shifted furthest towards the saddle, much like the case of quasimonochromatic noise in the adiabatic limit (Dykman 1990; see also section 6). In the presence of noise, therefore, there is an enhanced probability of an inter-well transition occurring once per half-cycle of the difference frequency, thereby effectively amplifying the heterodyne signal in very much the same way as a low-frequency additive signal can be amplified in conventional bistable SR.

The occurrence of NEH was sought and investigated experimentally using the circuit model shown in figure 33. It was driven by a signal $A_{\text {in }}^{\prime} \cos \left(\omega_{0}^{\prime}+\Omega^{\prime}\right) t^{\prime}$ and a multiplicatively applied reference signal $A_{\text {ref }}^{\prime} \cos \omega_{0}^{\prime} t^{\prime}$. Here $A_{\text {in }}^{\prime}$ and $A_{\text {ref }}^{\prime}$ are the amplitudes of the signals in volts, $f^{\prime}\left(t^{\prime}\right)$ is the actual value of the noise applied to the circuit, and $\omega_{0}^{\prime}$ and $t^{\prime}$ are the real frequency and time, respectively. The actual differential equation for the voltage $x^{\prime}$ in the circuit was of the form

$$
\begin{aligned}
R_{1} C_{1} \frac{\mathrm{d} x^{\prime}}{\mathrm{d} t^{\prime}}= & \frac{R_{9}}{R_{6}} \\
& \frac{R_{1}}{R_{3}} x^{\prime}-\frac{R_{9}}{100 R_{7}} \frac{R_{5} R_{1}}{R_{4} R_{3}} x^{\prime 3}+\frac{R_{9}}{10 R_{8}} \frac{R_{1}}{R_{3}} A_{\mathrm{ref}}^{\prime} x^{\prime} \cos \omega_{0}^{\prime} t^{\prime} \\
& +\frac{R_{1}}{R_{2}} A_{\mathrm{in}}^{\prime} \cos \left(\omega_{0}^{\prime}+\Omega^{\prime}\right) t^{\prime}+f^{\prime}\left(t^{\prime}\right)
\end{aligned}
$$

with

$$
\begin{aligned}
& R_{1}=R_{2}=R_{3}=R_{4}=R_{7}=R_{8}=10 \mathrm{k} \Omega \\
& R_{5}=R_{9}=50 \mathrm{k} \Omega \quad R_{6}=200 \mathrm{k} \Omega \quad C_{1}=30 \mathrm{nF} .
\end{aligned}
$$




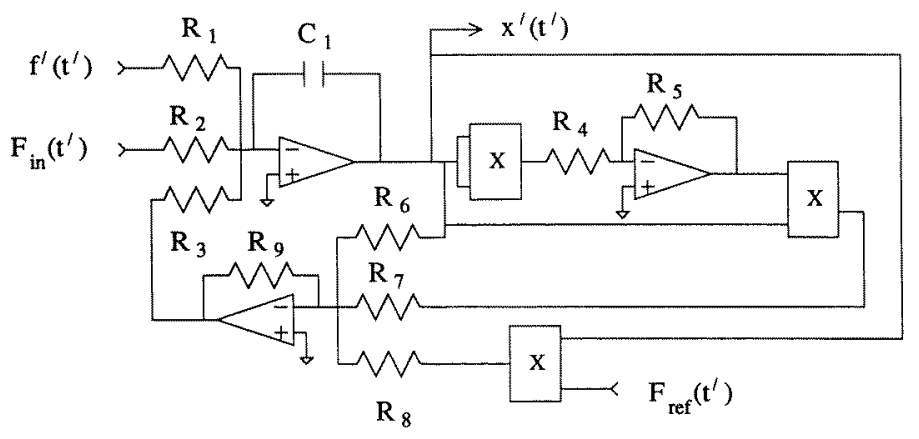

Figure 33. A block diagram of the electronic circuit used to investigate noise-enhanced heterodyning, modelling the motion of an overdamped particle (91) in the potential (30): $F_{\text {ref }}\left(t^{\prime}\right)=A_{\text {ref }}^{\prime} \cos \omega_{0}^{\prime} t^{\prime}$ and $F_{\text {in }}\left(t^{\prime}\right)=A_{\text {ref }}^{\prime} \cos \left(\omega_{0}^{\prime}+\Omega^{\prime}\right) t^{\prime}$; the noise input $f^{\prime}\left(t^{\prime}\right)$ can be either exponentially correlated quasi-white noise or high-frequency narrow-band noise (Dykman et al 1994a).

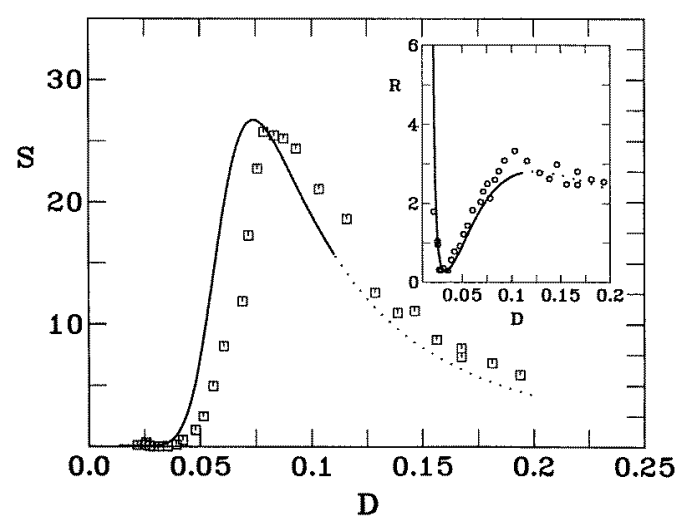

Figure 34. Normalized heterodyne signal $S$ and signal-to-noise ratio $R$ (inset) as functions of the intensity of the low frequency noise $D$ for $\omega_{0}=1.885, \Omega=0.008$ (Dykman et al 1994a). The squares represent the experimental data; the curves represent the theory, which has been dotted in the range of large $D$ where it is only qualitative.

After appropriate scalings (Dykman et al 1994a), and changing $x^{\prime} \rightarrow x$, (100) goes over into (91) for the potential (30). The circuit was driven by noise from a feedback shiftregister noise generator and by two sinusoidal periodic forces from Hewlett-Packard 3325B frequency synthesizers.

Measurements of the amplitude of the heterodyne signal $S=a^{2} / A_{\text {in }}$, and the SNR $R$ (98) and their dependences on noise intensity, are compared with the theoretical predictions (curves) based on (96) and (98) in figure 34. It is clearly seen that the dependence of the signal amplitude on $D$ is of the form of an asymmetric resonant curve, which is extremely steep on the small- $D$ side. This form is typical of SR (see section 4.2). For small $D$, where the system is effectively confined to one well of the potential, the signal magnitude corresponds in effect to the value it would have for heterodyning in a single-well nonlinear potential, and is extremely small. The dependence of the SNR on $D$ (inset) is also similar to that seen in stochastic resonance: for very small $D$ where the inter-well transitions do not come into play, $R$ decreases with increasing $D$, but then it displays a sharp increase. 
We notice that the maximum of $R(D)$ is shifted to higher noise intensities compared to that of the amplitude of the heterodyne signal. The experimental data are in good qualitative and quantitative agreement with the simple theory (curves) outlined above and described in more detail by Dykman et al (1994a). There are no adjustable parameters.

It follows from (94) and (98) that the SNR, for a given effective noise intensity $D$, should be proportional to the squared amplitudes of both the reference and input signals, and inversely proportional to the squared reference frequency $\omega_{0}$. These and several other predictions of the theory were observed to hold over a broad range of parameters (Dykman et al 1994a).

It is evident that, in close analogy to conventional SR, NEH in a bistable system can produce a very substantial enhancement of the heterodyne signal over that obtained by heterodyning in, for example, a single-well nonlinear system. The same is not true, however, of the SNR. Notwithstanding the huge noise-induced rises seen in $R(D)$ in NEH, and in conventional SR, the SNR does not quite reach the value it would have for the same $D$ in a single-well system. Nonetheless, we note that NEH offers a method by which the SNR can be protected against changes in the ambient noise level, if the average operating point is chosen to be very close to, but slightly beyond, the maximum in $R(D)$. In applications where it is important for the SNR to be stable, this could prove to be a useful feature. Another advantageous feature of NEH is that it provides high-frequency selectivity.

Building on the understanding gained from studies of the analogue electronic model, both SR and NEH were subsequently observed in a passive nonlinear optical system (Dykman et al 1995a,b).

\subsection{Noise-induced linearization}

Finally, in this section on SR, we discuss the closely related phenomenon of noise-induced linearization which was identified in the course of experiments on SR, using analogue electronic models (Dykman et al 1994b). The unexpected observation was that the signal distortion introduced by passage through a nonlinear system could usually be reduced by the addition at the input of external white noise of sufficient intensity. (For convenience, we will concentrate on the effects of white or quasi-white noise. Most of the discussion, however, is applicable qualitatively, and sometimes quantitatively, to the case of non-white noise, provided that its correlation time is shorter than the characteristic reciprocal frequencies of interest.) The scenario was found to hold experimentally for many different nonlinear systems, including monostable as well as bistable, underdamped as well as overdamped, chaotic as well as regular, and for signals of various shapes. Because the resultant linearized output $x(t)$ is inevitably noisy, we consider how the ensemble average $\langle x(t)\rangle$ of the output varies with relevant parameters, for example with the noise intensity at the input.

A simple example of the effect was obtained from the standard SR system (30) and (57) modelling overdamped Brownian motion. Consider the periodic signal before and after passage through the system. Some results are shown in figure 35 showing that for very weak noise the response is similar to a square wave (Dykman et al 1993f) but that, as the noise intensity increases, the original sinusoidal wave is gradually restored. If the sinusoidal signal is replaced with a sawtooth waveform, much the same behaviour is observed, as shown in figure 36 .

The basic idea of linearization by added noise is, of course, already familiar from specific observations and applications in science and engineering, for example the removal of digitization steps in the output of an ADC, the linearization of periodic signals in neurophysiological experiments (French et al 1972) or the linearization of the response 
INPUT

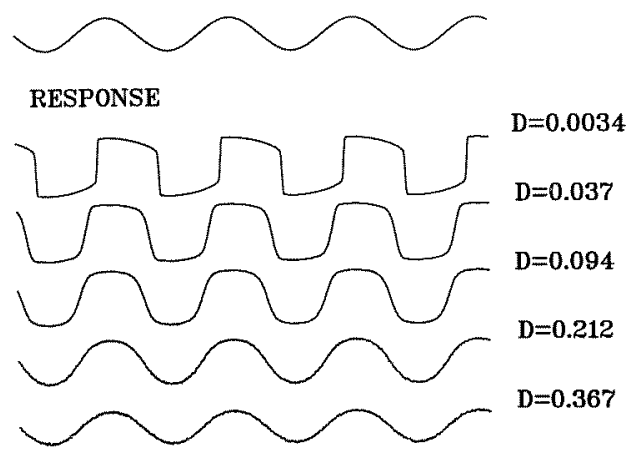

Figure 35. Noise-induced linearization for a sine wave passing through an electronic model of the overdamped double-well system given by equations (30) and (57). The periodic force at the input is shown in the upper trace. The ensemble-averaged response $\langle q(t)\rangle$, measured at the output, is shown for different noise intensities $D$ in the lower traces. The amplitudes of the latter have been normalized so as to be comparable with the amplitude of the force, for easier comparison of their relative shapes (Dykman et al 1994b).
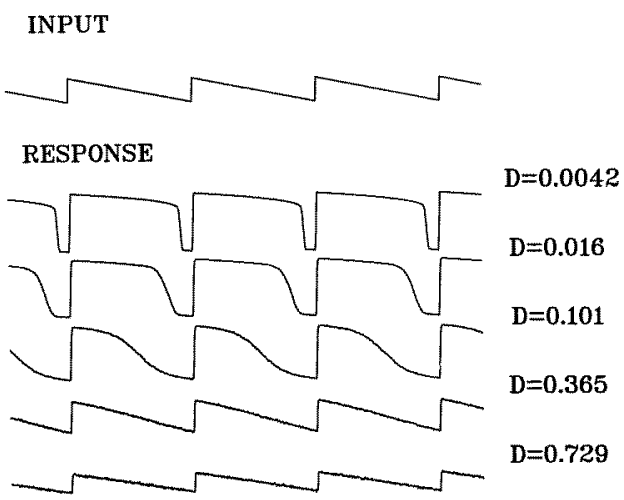

Figure 36. Noise-induced linearization for a sawtooth wave passing through an electronic model of the overdamped bistable system given by equations (30) and (57). The periodic force at the input is shown in the upper trace. The ensemble-averaged response $\langle q(t)\rangle$, measured at the output, is shown for different noise intensities $D$ in the lower traces. The amplitudes of the latter have been normalized so as to be comparable with the amplitude of the force, for easier comparison of their relative shapes (Dykman et al 1994b).

of ring-laser gyroscopes at low angular velocities (Vogel et al 1987a). The results under discussion suggested, however, that noise-induced linearization may exist as a more general phenomenon than had been appreciated, thus further illustrating the idea (Millonas 1996) that the role of noise in a dynamical system may often be, in a sense, creative.

Note that we use the word linearization in two rather different senses, and that these are exemplified by the results of figures 35 and 36 . The fact that a sinusoidal input can pass through the system without significant change of shape, as occurs for strong noise in the lowest trace in figure 35 , implies linearity in the direct sense, i.e. a proportionality between the amplitudes of output and input; this need not necessarily, however, imply that the constant of proportionality must be frequency independent. On the other hand, the results 
of figure 36, for a sawtooth waveform containing not only the fundamental frequency but also its higher harmonics, imply the occurrence of linearization in the 'Hi-Fi' sense that the system becomes non-dispersive within a certain frequency range when the noise intensity is large enough. In many cases of physical interest nonlinearity of the response arises because the frequency of the driving force (or its overtones) is close to some eigenfrequency of the system (or its overtones), or is related in a certain way to the relaxation time of the system. It is to be expected for such systems that linearization of the response in the sense of proportionality of output and input will not necessarily imply suppression of the frequency dispersion. (Obviously, one could try to devise special circumstances which might violate this rule. One possible example would be a logarithmic amplifier. However, even though this would be dispersion-free but highly nonlinear for low noise intensities, it seems likely nonetheless that the response would be eventually linearized by noise, just like the other cases that we consider.)

The physical origin of both forms of signal restoration can readily be understood, at least qualitatively, in the following terms. Where the amplitude response of a system to a periodic force is nonlinear, this arises because the amplitude of the vibrations induced by the force is comparable with, or larger than, some characteristic nonlinear length scale of the system. The scale in question (which may depend on frequency, see later) is determined by the structure of the region of phase space being visited by the system and by corresponding features in the dynamics. The effect of noise is to smear the system over a larger region of phase space, so that a variety of different scales and frequencies then become involved in the motion, even in the absence of periodic driving, and the effective characteristic scale will usually increase as a result. For sufficiently large noise intensities, therefore, the amplitude of the force-induced vibrations will become small compared to the scale (e.g. small compared to the average size of the noise-induced fluctuations), so that the nonlinearity of the response is correspondingly reduced. The system is then spending an increased proportion of its time far away from its attractor(s), at coordinate values where the timescale that characterizes the motion is in general quite different and sometimes shorter than that for small noise intensities. Consequently, there will be one or more ranges of frequency for which dispersion is likely to decrease (Stocks et al 1996). Although the linearization and the suppression of the dispersion arise, ultimately, through the same physical processes- the effect of noise in smearing the system over a larger region of its phase space-they do not necessarily become important at the same noise intensity. Dykman et al (1994b) and Stocks et al (1996) have given detailed analyses of noise-induced linearization phenomena in the standard SR system (30) and (57).

The harmonic oscillator provides a familiar example in which the two types of linearization are quite distinct: the response is always linear, the amplitude of the forced vibrations being proportional to the amplitude of the force; at the same time, dispersion can be very strong, particularly for an underdamped oscillator in the range of its eigenfrequency. For nonlinear systems, the narrow peaks in spectral densities of fluctuations are eventually (Dykman and Krivoglaz 1984) broadened by an increase in noise intensity; for underdamped systems, one of the basic broadening mechanisms comes about because the eigenfrequencies of vibration depend on amplitude, so that the broader the distribution over amplitude becomes, due to the increasing fluctuations, the broader becomes the distribution over eigenfrequencies (Dykman and Krivoglaz 1984, Dykman et al 1989, Dykman and McClintock 1992).

It is important to note, and it is clear from the above example, that the same physical processes which give rise to noise-induced linearization can also, under special circumstances, give rise to the opposite effect of noise-induced delinearization. The latter 
phenomenon is to be anticipated if the additional frequencies that become involved (as the result of an increase in noise intensity) resonate with the periodic force or with one of its harmonics, as in zero-dispersion phenomena (see section 4.3.2), or if the noise modifies the characteristic reciprocal relaxation time of the system so that it corresponds to the frequency of the periodic force, as in conventional stochastic resonance. In such cases, just as seen above for the system (30) and (57), it is to be expected that the promotion of nonlinearity by noise at intermediate intensities will be followed by the more general phenomenon of noise-induced linearization at still higher noise intensities owing to the usual noise-induced broadening of the relevant spectral peak(s). We have also observed and investigated sequential noise-induced delinearization/linearization phenomena in dynamical systems quite different from (30) and (57): cf the theoretical results cited and experimental results on an underdamped monostable oscillator discussed in section 4.3.2, as well as the results on the nonlinear response of such an oscillator (Dykman et al 1996a).

\section{Transient effects}

It often happens that one or more of the parameters characterizing a system change systematically with time. Consequently, the distributions of its coordinates and momenta also evolve with time. If a swept parameter changes slowly enough, the system may usually be regarded as remaining in quasi-equilibrium; the distributions are therefore almost the same as they would be for fixed values of the parameter in question. If the parameter changes relatively fast, however, this kind of adiabatic approximation is inapplicable because the system never has time to approach equilibrium, and a satisfactory description in general represents a difficult problem. Of particular interest is the situation that arises when the parameter is swept past some critical point of the system where a bifurcation occurs: critical slowing down means that, sufficiently close to the bifurcation, an adiabatic approximation will always fail. A well known physical example is that of a laser close to threshold, where the pump parameter moves past the critical value for the onset of lasing. In this section we consider two extreme situations, both of which have been investigated successfully through analogue electronic experiments. First, in section 5.1, we discuss an example of what happens when the swept parameter moves continuously past a critical value; then, in section 5.2, we consider an interesting phenomenon that occurs when the parameter is switched discontinuously from a sub-threshold to a super-threshold value, thereby placing the system transiently in an unstable state which subsequently decays.

\subsection{Swept-parameter systems}

Analogue experiments on symmetrical bifurcating swept-parameter systems (Stocks et al 1989c, 1990) and their comparison with the theories of Torrent and San Miguel (1988), Van den Broeck and Mandel (1987), Zeghlache et al (1989), Torrent and San Miguel (1988) and Torrent et al (1990) have already been reviewed in detail (Dykman et al 1994c) elsewhere. In this subsection we consider, instead, an example of an interesting phenomenon that occurs when the bifurcating system has a small-perhaps very small—asymmetry.

The investigations (Moss et al 1985a, Kondepudi et al 1986a, b) addressed the question of sensitivity in symmetry-breaking transitions, in quite general terms. They were motivated by attempts (Kondepudi and Nelson 1983, 1985) to account for the chiral symmetry of biological molecules and, in particular, to try to explain why the lower energy enantiomer has apparently been selected by evolution (Mason 1985), even though the energy difference between L- and D-amino acids is much smaller than $k_{\mathrm{B}} T$. The difference, due to 
parity-violating weak neutral current interactions (the so-called Z-force) is so small that, on purely energetic grounds, L-amino acids would be expected to outnumber their D-amino acid enantiomers by only 1 part in $10^{17}$; yet life is based on L-amino acids. As a possible explanation, Kondepudi and Nelson $(1983,1985)$ suggested that a very slow passage through a bifurcation could amplify a tiny effect of this kind because of the extreme sensitivity of the system to a small symmetry-breaking force when near the critical point. If $x$ is the amplitude of the solution that experiences symmetry-breaking then, by elimination of the fast modes (Haken 1977, Nicolis and Prigogine 1977), one can obtain an equation of the form

$$
\dot{x}=-A x^{3}+B\left(\lambda-\lambda_{c}\right) x+C g
$$

where $A, B$ and $C$ are constants that depend on the system, $\lambda$ is the control parameter that is to be swept and $g$ is a small interaction or bias that selects among the broken-symmetry states. Note that (101) is very general: its form is determined by the symmetry that is broken, not by details of the particular system (Guckenheimer and Holmes 1983).

The steady states of (101) are shown in figure 37. With $g=0$, and assuming weak additive noise, each of the supercritical branches

$$
x= \pm \sqrt{B\left(\lambda-\lambda_{\mathrm{c}}\right) / A}
$$

will be selected with equal probability as $\lambda$ is swept through $\lambda_{\mathrm{c}}$. When $g \neq 0$, however, the bifurcation is no longer of the pitchfork type where two stable solutions and an unstable one merge together; rather, the two stable branches are then separated by a minimum distance $3(\mathrm{Cg} / 2 \mathrm{~A})^{1 / 3}$. Far above or below the critical point, the displacement of the $x \simeq 0$ solution due to non-zero $C g$ is $\sim C g / B\left(\lambda-\lambda_{\mathrm{c}}\right)$. Thus, the effect of $C g \ll 1$ is much larger near the critical point than away from it. The theory (Kondepudi et al 1986a) predicts that state selection will be strongly enhanced by a slow sweep of $\lambda$ past $\lambda_{\mathrm{c}}$.
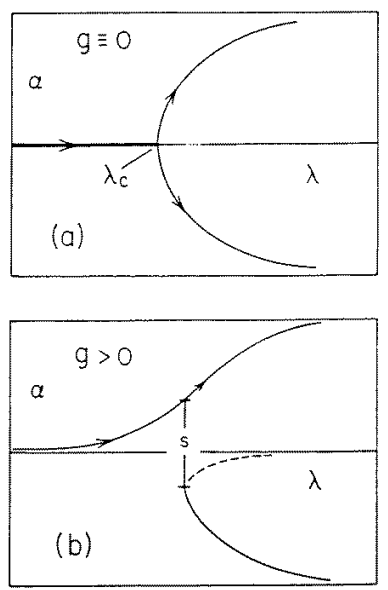

Figure 37. A pitchfork bifurcation showing: (a) perfect symmetry, with $g=0$; and (b) in the presence of a symmetry-breaking interaction with $g>0$ (Kondepudi et al 1986a).

The electronic model used (Kondepudi et al 1986a) for testing these ideas is shown diagrammatically in figure 38 ; for convenience, $\lambda_{\mathrm{c}}$ was set to zero. The voltage representing the changing parameter $\lambda(T)$ was derived from a sweep generator, and a switch was used to short circuit the integrator and set $x=0$ prior to the start of each sweep. Small nonidealities in the components meant that it was impossible to set $g=0$ rigorously: the 


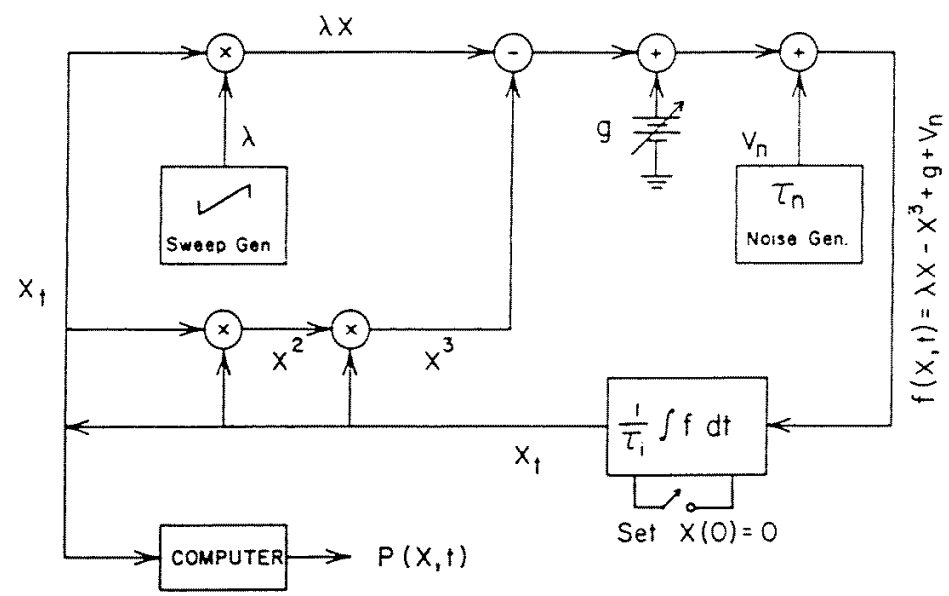

Figure 38. A block diagram of the electronic circuit model of (101). The shorting switch on the integrator can be closed with a pulse at $t=0$ to set $x(0)=0$ but it is normally open (Kondepudi et al 1986a).

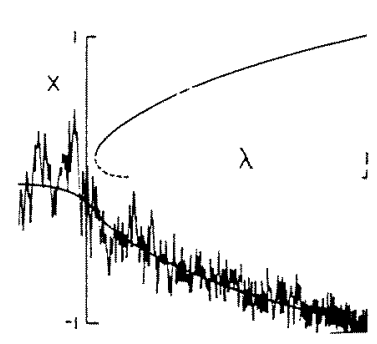

(a)

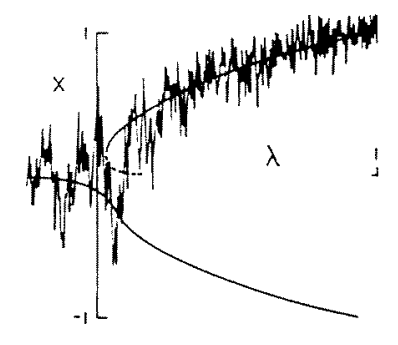

(b)

Figure 39. Example $x(t)$ trajectories measured for $g=-0.016, V_{n}=0.50 \mathrm{~V}$. Negative branch selection as in (a) is about 8.3 times as probable as positive branch selection as in (b), in this example. The full curves are the measured deterministic steady states (Kondepudi et al 1986a).

smallest value achievable in practice was $g \simeq 0.003 \mathrm{~V}$ which was, however, almost an order of magnitude smaller than the smallest value that was set explicitly.

Two typical trajectories measured for the swept system are shown in figure 39: the lower of these is more probable than the upper. To obtain the evolution of the probability distribution, 5000 such trajectories were collected and ensemble averaged at different fixed times, yielding the results shown in the lower part of figure 40. The upper evolution shows, for comparison, what happens when $\lambda$ is not swept, but the system is just released at $x=0$ in the presence of noise. The selectivity $S$ of the more probable state has been increased from 1.7 (static release) to 21.6 (swept $\lambda$ ). A comparison of measured and calculated values of $S$ for $g=0.016, \epsilon^{1 / 2}=0.133$, an integrator time constant of $\tau_{\mathrm{i}}=100 \mu \mathrm{s}$ and different sweep rates is given in table 1 (Kondepudi et al 1986a); the theory is only applicable when the sweep rate is fast enough for the probability density to be approximated by a drifting Gaussian. The agreement between theory and experiment may be regarded as satisfactory. 


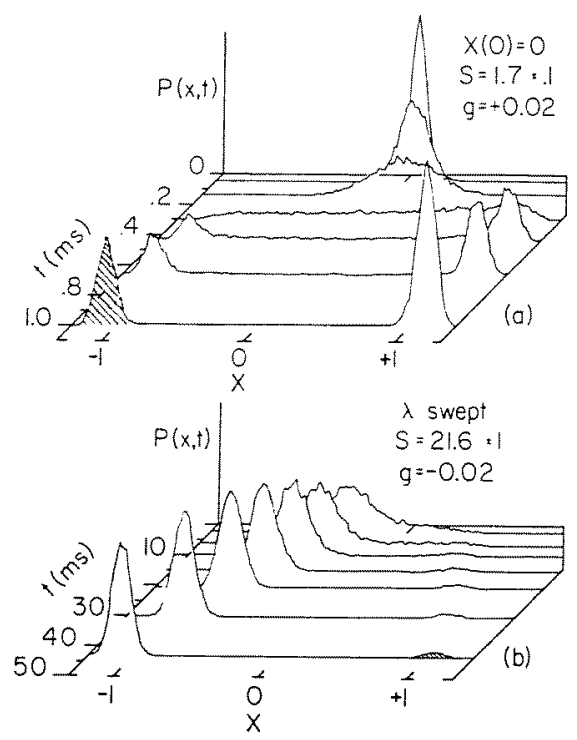

Figure 40. Measured time-evolving probability distributions $P(x, t)$ for two different sets of circumstances. (a) The unswept system, with fixed $\lambda$, setting $x=0$ at $t=0$. (b) Under sweptparameter conditions, sweeping $\lambda$ from $-1 \mathrm{~V} \rightarrow+1 \mathrm{~V}$ at the rate $32 \mathrm{~V} \mathrm{~s}^{-1}$. The same noise level applied in both cases and the same magnitude of $g$ was used, though its sign was reversed to illustrate selection of both branches. Note that the selectivity $S$ was very much larger in case (b) with the swept parameter (Kondepudi et al 1986a).

Table 1. Selectivity as a function of inverse sweep rate.

\begin{tabular}{lcl}
\hline $\begin{array}{l}\text { (Sweep rate })^{-1} \\
\left(\mathrm{~s} \mathrm{~V}^{-1}\right)\end{array}$ & Measured $S$ & Calculated $S$ \\
\hline 0.023 & 5.54 & 4.27 \\
0.046 & 6.53 & 5.80 \\
0.092 & 8.24 & 8.43 \\
0.174 & 9.12 & 12.88 \\
0.348 & 13.5 & NA \\
0.700 & 18.6 & NA \\
\hline
\end{tabular}

Hegstrom and Kondepudi (1990) have outlined a scenario with a constant flow of reactants into a pool of area $10 \mathrm{~km}^{2}$ and of several metres depth. Over a period of 50000100000 years, there would have been a $98 \%$ chance that the Z-force would have caused selection of the lower energy enantiomer, so life would then have evolved in that form. Whether or not symmetry breaking actually occurred at the pre-biotic stage, and the nature of the process(es) through which it occurred, continue to be vigorously debated (Cline 1996) but the electronic experiments show beyond reasonable doubt that the underlying mechanism proposed by Kondepudi and Nelson $(1983,1985)$ does work. It is not strongly influenced by correlation time, in the case of exponentially correlated coloured noise (Kondepudi et al 1986b), and it is potentially applicable in a variety of scientific and technological contexts. 


\subsection{Decay of unstable states and transient multimodality}

If a control parameter, for example $\lambda$ in (101) with $C g=0$, is changed discontinuously from below to above its critical value, the system will find itself in an unstable state. How this state subsequently decays will obviously depend on the nature of the system under study, and on the kind and intensity of the noise. A convenient way of characterizing the decay is in terms of nonlinear relaxation times (NLRTs) which are defined in terms of the transient moments. For a process $x(t)$ defined by a Langevin-like equation of the general form

$$
\dot{x}=v(x, t)+g(x, t) \xi(t)
$$

the NLRT associated with the average (over realizations of the noise $\xi(t)$ ) of a quantity $\phi(x)$ is defined (Binder 1973, Rácz 1976) by

$$
T_{\phi}=\int_{0}^{\infty} \frac{\langle\phi(t)\rangle-\langle\phi\rangle_{\mathrm{st}}}{\langle\phi\rangle_{i}-\langle\phi\rangle_{\mathrm{st}}} \mathrm{d} t .
$$

Here, $\langle\phi\rangle_{i}$ is the average value of $\phi$ in the initial state, $\langle\phi(t)\rangle$ is the average value of $\phi$ during the evolution from a given initial distribution to the final stationary distribution, where the average value of $\phi$ is given by $\langle\phi\rangle_{\mathrm{st}}$. Depending on the particular problem and for appropriate choices of the quantity $\phi(x)$, this may be a definition of a certain global time scale for the relaxation of initial conditions towards the steady state. The interest of this definition lies in the fact that the NLRTs can be usually calculated via techniques based on knowledge of the evolution operator of the probability densities. The formalism can be systematized in a way which parallels the calculation of the first passage time (FPT) moments and has turned out to have some practical and theoretical advantages over them. Its usefulness has been checked in the context of white noise (Jiménez-Aquino et al 1988, Casademunt et al 1989a), but the advantages have been found particularly marked in the case of coloured-noise problems (Casademunt and Sancho 1989).

The NLRT has been measured and investigated in analogue electronic experiments (Casademunt et al 1989c). It was studied for both white noise and (exponentially correlated) coloured noise, fixed initial conditions and distributed initial conditions; and in the latter case, for coloured noise, a distinction was made between coupled initial conditions where a correlation exists between fluctuations just before and after the change in the control parameter, and uncoupled initial conditions where the noise sources before and after the change are uncorrelated. These experiments (Casademunt et al 1989c), and their comparison with the body of theory introduced by the Barcelona group (Casademunt and Sancho 1989, Casademunt et al 1989a, b) have been reviewed by Dykman et al (1994d), and need not be discussed further here. It is worth emphasizing, however, that the analogue experiments were very readily able to provide all the permutations of noise and initial conditions that were required.

Following Iwaniszewski et al (1994), we now describe investigations of an interesting phenomenon, transient multimodality (TM), that can occur during the decay of an unstable state. In TM, there is a period during the evolution of the system when the number of maxima in the probability distribution is larger than it is in either the initial or final state, i.e. for a sizeable interval of time, the system can be found with comparable probability in any one of a number of states that is larger than the number of its steady states. Such behaviour was first reported by Baras et al (1983), Frankowicz and Nicolis (1983) and Frankowicz et al (1984) in theoretical studies of explosive chemical reactions and combustion. Later on the possibility of TM was mentioned for some optically bistable systems (Broggi and Lugiato 1984a, b, Broggi et al 1985; Lugiato et al 1986) and for Brownian particles in 
shear flows (Wio and Zanette 1993). The theoretical predictions were confirmed by means of numerical simulations (Baras et al 1983, Frankowicz and Nicolis 1983, Broggi and Lugiato 1984a, b, Broggi et al 1985, de Pasquale and Mecozzi 1985, Mitschke et al 1985) and experiments on optical bistability (Lugiato et al 1986, Mitschke et al 1985, Lange et al 1985, Lange 1988, Nalik et al 1989), an electronic circuit (Mitschke et al 1985), a laser with a saturable absorber (Arimondo et al 1987), a semiconductor laser (Spano et al 1990), electrohydrodynamic convection in nematic liquid crystals (Kai et al 1992), combustion processes (Lemarchand et al 1989) and also through studies of the dynamics of error growth in numerical calculations (Nicolis and Nicolis 1991).

The usual theoretical description associates TM with evolution in a potential with a flat plateau, for which critical slowing down occurs (Broggi and Lugiato 1984a, Broggi et al 1985). The fluctuations driving the system accelerate the evolution of some stochastic realizations over the flat region, after which the system is rapidly switched to the vicinity of a stable state $x_{\mathrm{s}}$. Since some stochastic realizations still remain at the plateau region one observes the bunching of trajectories in two places, namely at the plateau and in the bottom of the potential well. Thus the probability distribution possesses two maxima. This phenomenon arises some time after the initial probability distribution peak reaches the plateau and it disappears again once an appropriate mass of probability distribution (i.e. a sufficient proportion of stochastic trajectories) has left the flat part of the potential.

Such behaviour can occur (Valle et al 1990, Colet et al 1991) if the evolution of a system is characterized by two different time scales. The longer one results from the long induction stage at the potential plateau. It is of the order of the mean first passage time (MFPT) $T$ needed to reach the edge of the flat region. However, because of the essential role played by fluctuations at this stage of the evolution, the actual value of this time for a given realization is distributed within an interval of the order of the variance $\Delta T$ of the MFPT, which is of the same order as $T$. The shorter time scale $t_{\mathrm{d}}$ is associated with the deterministic transit from the edge of the plateau to the bottom of the potential. In terms of these time scales the type of evolution can be characterized by the ratio $\eta=t_{\mathrm{d}} / \Delta T$. TM is possible provided $\eta<1$. In contrast, if $\eta>1$, most of the stochastic trajectories leave the plateau region before any of them reach the vicinity of $x_{\mathrm{s}}$. Thus the probability of finding the system somewhere between the plateau and the bottom is large and, consequently, the probability distribution peak travels continuously towards its stationary position. The relation $\eta<1$ is known (Caroli et al 1980) to apply close to a marginal state $\left(U^{\prime}(x)=U^{\prime \prime}(x)=0\right)$ but not in the vicinity of an arbitrary unstable state $x_{\mathrm{u}}\left(U^{\prime}\left(x_{\mathrm{u}}\right)=0, U^{\prime \prime}\left(x_{\mathrm{u}}\right)<0\right)$. Hence, some authors (Broggi et al 1985; Arimondo et al 1987, Colet et al 1991) conclude that, generally, TM is not expected during relaxation from the top of a potential barrier. The parabolic curvature of the barrier does not generate two different time scales if the noise intensity is not small (otherwise there arises the logarithmic Suzuki time (Suzuki 1976)). It should be noted, however, that this conclusion stemmed from the analysis of only one type of potential, namely the quartic potential

$$
U(x)=\frac{1}{4} b x^{4}+\frac{1}{2} a x^{2}
$$

(with $b>0, a<0$ ) that is usually considered while dealing with the decay of an unstable state (e.g. Suzuki 1976).

Iwaniszewski (1992a) showed, however, that TM should also arise during evolution from an unstable state $x_{\mathrm{u}}$. This effect applies to a very wide class of systems, even in cases very far from marginality. It turns out that the type of evolution (continuous or TM) to be anticipated depends only on the sign of the fourth derivative of the potential in the 
unstable state-if it is negative some new peaks must appear before the initial maximum at $x_{\mathrm{u}}$ disappears. However, it also means that the potential's curvature at the unstable state is much steeper than in the parabolic case, so that the system should leave the vicinity of $x_{\mathrm{u}}$ very quickly. We now consider how to reconcile this result with the earlier discussion in terms of time scales.

Iwaniszewski et al (1994) considered a one-dimensional overdamped oscillator subject to the force derived from a double-well potential and a Gaussian white noise. Its evolution is governed by the Langevin equation

$$
\dot{x}=-U^{\prime}(x)+\xi(t)
$$

with a symmetrical sixth-order polynomial potential

$$
U(x)=\frac{1}{6} c x^{6}+\frac{1}{4} b x^{4}+\frac{1}{2} a x^{2} .
$$

The parameter $a<0$ fulfils the requirement of instability at $x_{\mathrm{u}}=0, b$ determines the sign of $\left.\frac{\mathrm{d}^{4} U}{\mathrm{~d} x^{4}}\right|_{x=0}$ and $c>0$ ensures the existence of stable states $\pm x_{\mathrm{s}}$. The noise $\xi(t)$, of zero mean, is characterized by its correlation function

$$
\left\langle\xi(t) \xi\left(t^{\prime}\right)\right\rangle=2 D \delta\left(t-t^{\prime}\right)
$$

where $D$ is the noise strength.

Analogue experiments were used to confirm that TM really occurs during the decay of an unstable state. The circuit used to model (106)-(108) is shown schematically in figure 41 (cf Casademunt et al 1989c). Two independent Gaussian, exponentially correlated noises $\Xi$ and $Z$ are supplied from feedback shift-register noise generators. Since the noises' correlation times are of the order of one tenth of the time constant of the Miller integrator, they are perceived as effectively white noises with correlation functions

$$
\begin{aligned}
& \left\langle\Xi(\tau) \Xi\left(\tau^{\prime}\right)\right\rangle=2 \theta_{\xi} V_{\xi}^{2} \delta\left(\tau-\tau^{\prime}\right) \\
& \left\langle Z(\tau) Z\left(\tau^{\prime}\right)\right\rangle=2 \theta_{\zeta} V_{\zeta}^{2} \delta\left(\tau-\tau^{\prime}\right)
\end{aligned}
$$

where $V_{\xi}^{2}$ and $V_{\zeta}^{2}$ are the variances of the noise voltages. A square-wave signal periodically operates a solid-state switch S (DG303ACJ) to alter the system between two versions of the circuit. The first one (disconnected in figure 41) prepares the required distribution of initial conditions and it operates within a linear Langevin equation

$$
\frac{\mathrm{d} V_{x}}{\mathrm{~d} \tau}=-\lambda V_{x}+Z_{\zeta}(\tau)
$$

Since the half-period of the switching signal is much longer than the relaxation time of this circuit, the stationary distribution of (111), namely a Gaussian distribution centred at $V_{x}=0$, is generated. During the second half-period the main circuit comes into action (see figure 14) giving

$$
\frac{\mathrm{d} V_{x}}{\mathrm{~d} \tau}=-\gamma V_{x}^{5}-\beta V_{b} V_{x}^{3}-\left(\alpha_{1} V_{a}+\alpha_{2} V_{b}^{2}\right) V_{x}+\Xi(\tau) .
$$

All of the parameters indicated by small Greek letters in equations (109)-(112) can be expressed $[25,26]$ in terms of component values in the electronic system.

After the scalings $V_{x}=A x$ and $\tau=B t,(109)$ and (112) yield the required equations (106)-(108) while equations (110) and (111) guarantee a Gaussian initial distribution with a variance $\rho_{0}$. The four voltages $V_{a}, V_{b}, V_{\xi}$ and $V_{\zeta}$ are set externally to vary the parameters of the potential $U(x)$, the noise strength $x$ and the width $\rho_{0}$ of the initial distribution.

The output, a time-dependent voltage $V_{x}(t)$, was analysed by means of a Nicolet NIC-80 computer system. For each realization of $x(t)$ an initial state was first prepared according 


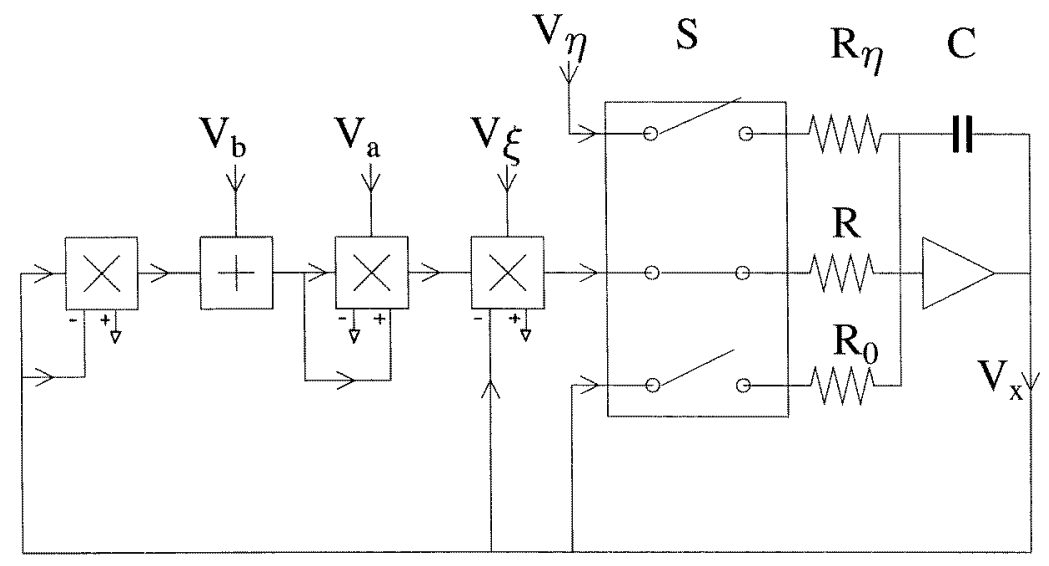

Figure 41. A block diagram of the electronic circuit used to investigate the evolution of equation (106) with randomly distributed initial conditions. The signals connected to the top of the multipliers are added to the results of the multiplication (Iwaniszewski et al 1994).

to (111) with the required statistics (an appropriate value of $\rho_{0}$ ). Then, as the system was switched to the main circuit, the Nicolet ADC was triggered and an input sweep was acquired: 1024 values of $V_{x}(t)$ were digitized and recorded at equal time intervals, with 12-bit precision. The sweep was analysed by examining each 16th value and incrementing the corresponding point of a 64-level distribution. The process then repeated, building up a set of 64 64-point distributions separated by equal intervals of time, and was continued until the statistics were adequate. It turned out that $2 \times 10^{4}$ realizations were sufficient.

The results obtained confirmed immediately the existence of TM in (106)-(108). An example of the time evolution of the probability distribution $W(x, t)$ for $b<0$ is exhibited as a three-dimensional plot in figure 42(a). The existence of a time regime within which three probability distribution peaks coexist is clearly evident. A comparison of the experimental histograms of the instantaneous probability distribution for several different fixed times is shown in figure 42(b). Notwithstanding the statistical fluctuations in the shape of $W(x, t)$, the trimodal character of the probability distribution at intermediate times is clearly demonstrated.

Iwaniszewski (1992b) showed that, for the potential (107), TM can appear not only for $b<0$ but also for some positive values of $b$, namely for $b<b_{\mathrm{c}}=\sqrt{-\frac{5}{3} a c}$. An example of the evolution of the probability distribution measured for positive $b$ is given in figure 43 . It is seen, that, within the experimental accuracy, the evidence of TM is very weak. Over a comparatively long period of time, the probability distribution remains very flat, almost uniform, and the difference between the heights of the maxima and minima is very small, so that the effect of TM is rather insignificant. As the value of $b$ was reduced from $b_{\mathrm{c}}$ the phenomenon of TM became more clearly resolved, although it lasted for a very short time. As $b$ crossed zero the coexistence of three probability distribution peaks became evident and the TM persisted for a very long time. Such behaviour agrees well with the previous analysis of Iwaniszewski (1992b) which distinguished short life $\left(0<b<b_{\mathrm{c}}\right)$ and long life $(b<0)$ forms of TM. In the former case, the properties of the potential determine the finite time of coexistence of the three peaks, while in the latter case it is only the strength of the 


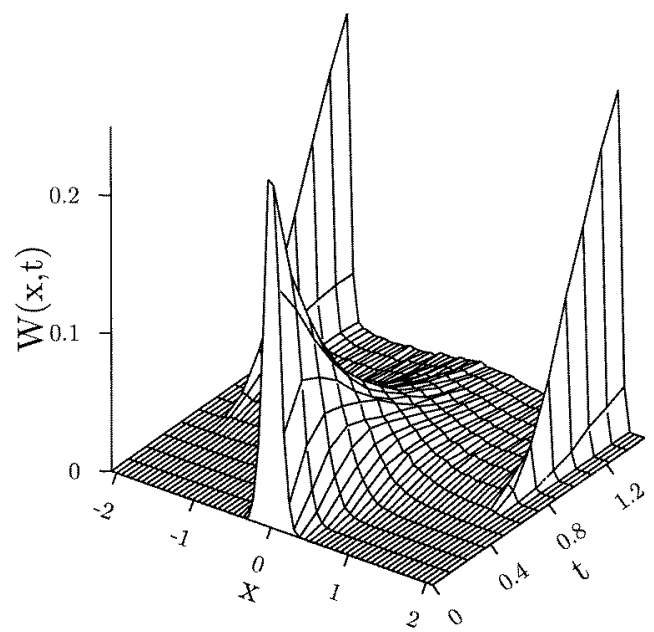

(a)

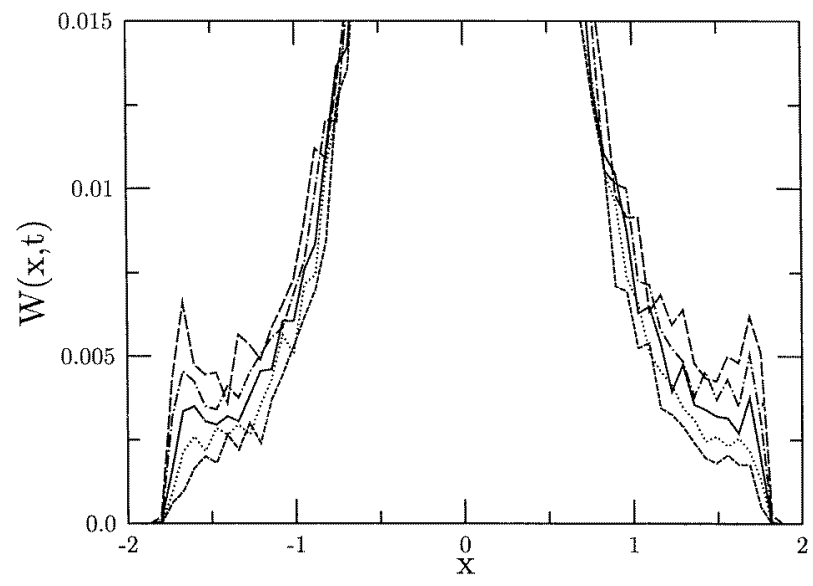

(b)

Figure 42. Time evolution of the probability distribution $W(x, t)$ for $a=-2, b=-2.47$, $c=1, \rho_{0}=0.01$ and $D=0.02$ : (a) a three-dimensional plot; and (b) a plot of the lower part of the instantaneous probability distribution for $t=0.56$ (short-dashed curve), 0.58 (dotted curve), 0.60 (full curve), 0.63 (dot-dashed curve) and 0.65 (long-dashed curve) (Iwaniszewski et al 1994).

noise that determines the time at which the middle peak disappears. Hence, if $q$ is very small, the effect will be observed over rather a long period and TM eventually seems to disappear because of the experimental indistinguishability of the middle maximum and the minima.

Iwaniszewski (1992b) also showed that both sources of randomness, i.e. the random initial conditions and the stochastic force, affect the appearance of TM. The simpler case for theoretical treatment is that of deterministic evolution with a Gaussian distribution over initial states of width $\rho_{0}$. He performed some calculations for small $\rho_{0}$. In particular, he derived formulae for the critical time $t_{\mathrm{cr}}$ and position $x_{\mathrm{cr}}$ of the appearance of new maxima 


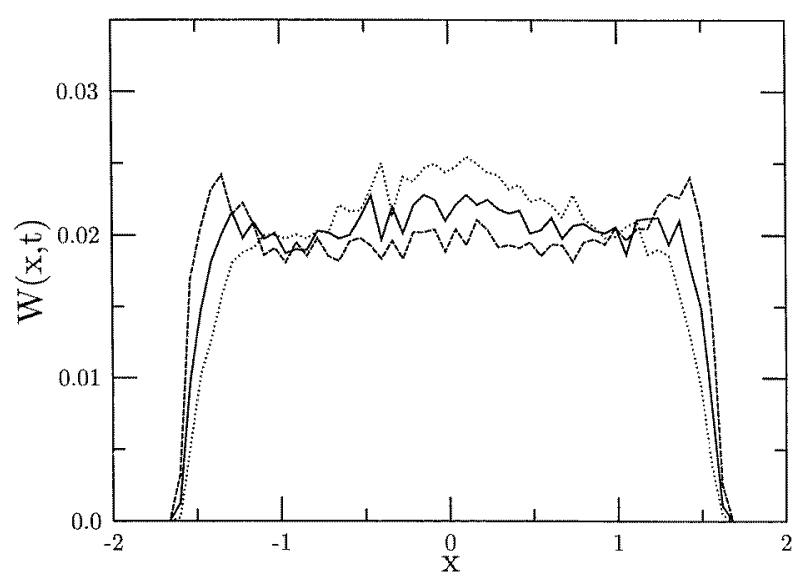

Figure 43. Cuts through the probability distribution $W(x, t)$ for $a=-2, b=0.99, c=1$, $\rho_{0}=0.01$ and $D=0$, at times: $t=0.93$ (dotted curve), 0.99 (full curve), and 1.04 (dashed curve) (Iwaniszewski et al 1994).

(i.e. values which satisfy $\left.W^{\prime}\left(x_{\mathrm{cr}}, t_{\mathrm{cr}}\right)=W^{\prime \prime}\left(x_{\mathrm{cr}}, t_{\mathrm{cr}}\right)=0\right)$

$t_{\mathrm{cr}}^{2} \approx-\frac{1}{2 a} \ln \left[\rho_{0}\left(-5 \frac{c}{a} x_{\mathrm{cr}}^{2}-3 \frac{b}{a}\right)\right]+\frac{1}{2 a\left(x_{\mathrm{cr}}^{2}+p\right)}\left[x_{\mathrm{cr}}^{2} \ln \left(1+\frac{x_{\mathrm{cr}}^{2}}{p}\right)+p \ln \left(1-\frac{x_{\mathrm{cr}}^{2}}{x_{s}^{2}}\right)\right]$

$x_{\mathrm{cr}}^{2} \approx-\frac{13 b}{20 c}+\frac{7}{20} \sqrt{\left(\frac{b}{c}\right)^{2}-\frac{200 a}{49 c}}$

where the stable states $\pm x_{\mathrm{s}}$ of the potential $U(x)$ are given as

$$
x_{\mathrm{s}}^{2}=-\frac{b}{2 c}+\frac{1}{2 c} \sqrt{b^{2}-4 a c}
$$

and

$$
p=\frac{b}{2 c}+\frac{1}{2 c} \sqrt{b^{2}-4 a c} .
$$

These formulae, which approximate well the exact numerical data of Iwaniszewski (1992b), are compared with the experimental data in figures 44 and 45 for several values of $\rho_{0}$ $(D=0)$. The dependence of $x_{\mathrm{cr}}$ on the initial distribution width $\rho_{0}$ is small compared to the experimental inaccuracy, so only the experimental data for $\rho_{0}=0.01$ are shown on figure 44. Due to some slight asymmetries in the circuit the probability distribution is not exactly symmetric, and so the positions of left and right critical points are indicated separately; the averages of the left and right critical time values are shown by the data in figure 45.

The experimental uncertainties in the shape of $W(x, t)$ make it impossible to identify the exact time of appearance of a new maximum. To be sure that a given small hump is indeed a new probability distribution peak, and not just a fluctuation, the value of $t_{\mathrm{cr}}$ is slightly overestimated. Thus, the experimental data in figure 45 are slightly greater than the analytic ones. Similarly, since a new maximum tends almost immediately after its appearance to the vicinity of its stationary position (see figure 3 in Iwaniszewski 1992b), the experimental values of $x_{\mathrm{cr}}$ are a little higher than the theoretical ones. Another source of error, believed to be unimportant, must come from the finite grid of voltage values-the 


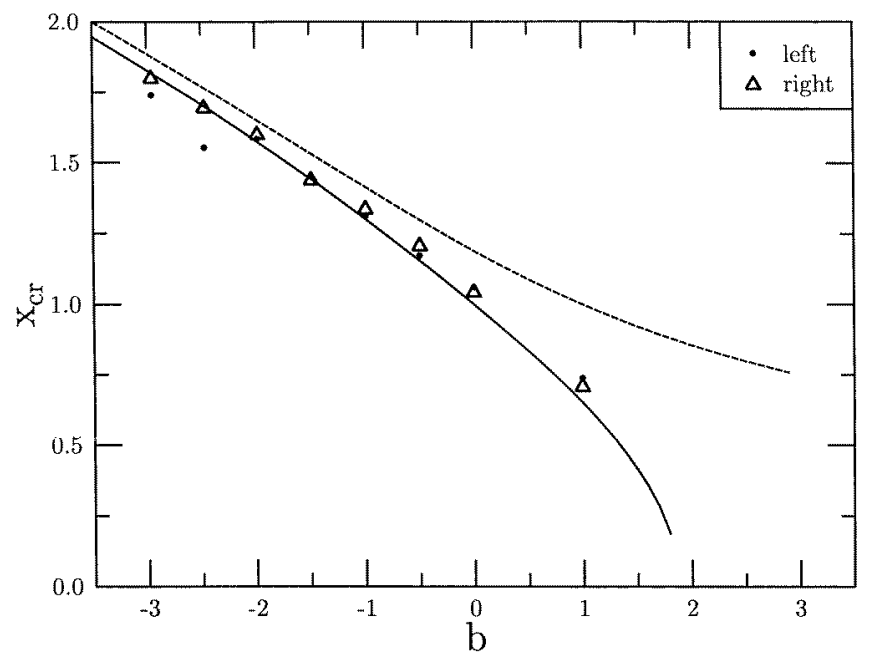

Figure 44. The absolute value of the position $x_{\mathrm{cr}}$ at which new extrema appear, plotted as a function of $b$ for $a=-2, c=1, \rho_{0}=0.01$ and $D=0$. The full curve represents the theory of equation (114) and the data points represent the experimental results for the extrema located to the left (dots) and to the right (triangles) with respect to the unstable point $x_{\mathrm{u}}$. The position of the stationary maximum $x_{\mathrm{s}}(115)$ is shown by the broken curve (Iwaniszewski et al 1994).

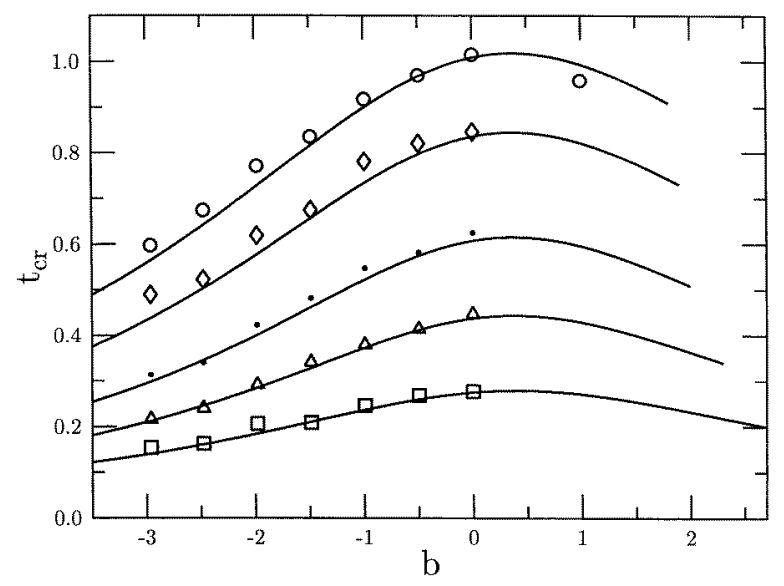

Figure 45. The time $t_{\mathrm{cr}}$ at which new extrema appear, plotted as a function of $b$ for $a=-2$, $c=1$ and $q=0$. The full curve represents the theory of equation (113) and the data points represent the experimental results, taken as the average of the times at which the left and right maxima appear. The variance of the initial distribution, from top to bottom, is $\rho_{0}=0.01,0.02$, $0.05,0.10$ and 0.20 (Iwaniszewski et al 1994).

whole range of voltages (which was about $15-20 \%$ greater than the distance between the stationary maxima) was divided into 64 intervals. Despite the non-ideality introduced by this coarse-graining, remarkably good agreement was obtained between the theoretical and experimental results.

Iwaniszewski et al (1994) go on to consider in detail the relationships between the shape of the potential, time scales an the possibility of TM during relaxation from an unstable 
state. They also discuss the relationship between marginality and TM. The analogue model, and its observed behaviour, underpinned and motivated the discussion by tying the theory closely to what could be observed in an actual experiment.

\section{Phenomena induced by quasimonochromatic noise}

Most of the work discussed in the preceding sections has related to the effects of white noise (WN) on nonlinear systems-effects that stem from the analysis pioneered by Einstein (1905) and Smoluchowski (1906). However, as previously remarked, WN is an idealization. In the real world, noise is seldom white. (Recall that, even in WN experiments, the WN has to be replaced by exponentially correlated noise $(\mathrm{ECN})$ whose correlation time is very short compared to all characteristic times of the system under study.) It is, therefore, necessary to establish what happens when the noise is coloured, i.e. has a power spectrum that cannot be considered to be flat within the frequency range of interest.

In the last decade there have been many attempts to investigate the effects induced by non-white noises. The majority of these have been devoted to ECN, and often this very specific type of noise has been referred to just as 'coloured noise'. Detailed reviews of the earlier work can be found in the articles by Sancho and San Miguel (1989), Lindenberg et al (1989a, b), Grigolini (1989), Hänggi (1989), Brand et al (1989) and Dykman and Lindenberg (1994). The noise colour significantly enriches the fluctuation dynamics, particularly where the characteristic correlation time of the noise is not small compared to the relaxation time of the system. In many physical situations, however, the driving noise is more complicated than ECN. In particular, its power spectrum may be peaked near some characteristic frequency or frequencies, and it is obviously important to investigate whether and how such structure may influence the response of the system. In fact, it would be reasonable to save the term 'coloured' just for such a noise, as the most common example of it is the incoherent electromagnetic field of light with a given colour. In what follows we call it quasimonochromatic noise (QMN).

The occurrence of unusual effects in fluctuations of systems driven by quasimonochromatic noise was predicted, and many of these effects were analysed in Dykman (1990). We will now discuss the effects of QMN and their observation through analogue simulations.

\subsection{Quasimonochromatic noise and its generation}

The simplest form of the QMN is the noise with the power spectrum (Dykman 1990)

$$
\Phi(\omega)=\frac{4 \Gamma D}{\left(\omega^{2}-\omega_{0}^{2}\right)^{2}+4 \Gamma^{2} \omega^{2}} \quad \Gamma \ll \omega_{0} .
$$

Such noise corresponds to fluctuations of the coordinate of an harmonic oscillator of frequency $\omega_{0}$

$$
\ddot{f}(t)+2 \Gamma \dot{f}(t)+\omega_{0}^{2} f(t)=\xi(t)
$$

driven by white noise $\xi(t)$ with correlator

$$
\left\langle\xi(t) \xi\left(t^{\prime}\right)\right\rangle=4 \Gamma D \delta\left(t-t^{\prime}\right) .
$$

Following traditional notations, we define the noise power spectrum (117) without the factor of $2 \pi$ used in (53) for the spectral density of fluctuations of a dynamical system. In cases where the noise originates from a coupling between the oscillator and a heat bath, the characteristic noise intensity $D$ represents the temperature. Note that the noise with the 
spectrum (117) is also called harmonic noise (Igarashi and Munakata 1988, Ebeling and Schimansky-Geier 1989, Schimansky-Geier and Zülicke 1990), usually referring to a range where $\Gamma$ and $\omega_{0}$ are of the same order of magnitude; we shall reserve QMN to describe the limit $\Gamma \ll \omega_{0}$ where some of the most interesting new effects occur. Fluctuations of this general type are seen in a wide variety of physical systems, for example off-shore structures subject to waves, systems coupled to localized vibrations, in solids or large molecules.

The power spectrum (117) of QMN, plotted in figure 46 for a particular choice of parameters, is obviously very different from that of WN, which would be flat, or of ECN, which would decrease with frequency $\omega$ as $\left(1+\omega^{2} \tau_{c}^{2}\right)^{-1}$, where $\tau_{c}$ is the correlation time of the noise. Not surprisingly, therefore, the effect of $\mathrm{QMN}$ on physical systems can be very different from that of WN or of ECN, and has been shown to possess some distinctive features (Dykman 1990, Dykman et al 1991b, 1993e, Millonas and Dykman 1994, Einchcomb and McKane 1995). This is especially true in relation to bistable systems, including the simplest one, the symmetrical overdamped system

$$
\dot{x}+U^{\prime}(x)=f(t) \quad U(x)=-\frac{1}{2} x^{2}+\frac{1}{4} x^{4}
$$

that we have already considered in several different contexts. Analogue techniques are in many ways ideal for investigating problems involving QMN because they cope relatively easily with the widely differing time scales involved. Circuit models of the type described above can be used. The only differences from the methods discussed previously are that the driving noise must of course be QMN rather than WN and that, as we shall see, it is sometimes necessary to analyse the $x(t)$ data in a different way. The necessary QMN is readily created experimentally by 'filtration' of quasi-white noise through an harmonic oscillator. A suitable circuit (Dykman et al 1993e) is sketched in figure 47. In this example, the integrators had equal time constants $\tau_{\mathrm{I}}=R_{3} C_{1}=R_{2} C_{2}=1 \mathrm{~ms}$. Because the quasiwhite input noise $\xi(t)$ had a correlation time $\tau_{\mathrm{N}}=4.53 \mu \mathrm{s} \ll \tau_{\mathrm{I}}$, it was perceived by the circuit as white, with characteristic intensity

$$
D=\frac{1}{2 \Gamma} \frac{\left\langle V_{\mathrm{N}}^{2}\right\rangle \tau_{\mathrm{N}}}{\tau_{\mathrm{I}}}
$$

where $\left\langle V_{\mathrm{N}}^{2}\right\rangle$ was the mean-square ECN noise voltage at the input. The output $f(t)$ from the circuit was QMN with the spectrum (117). Its parameters were nominally given by the

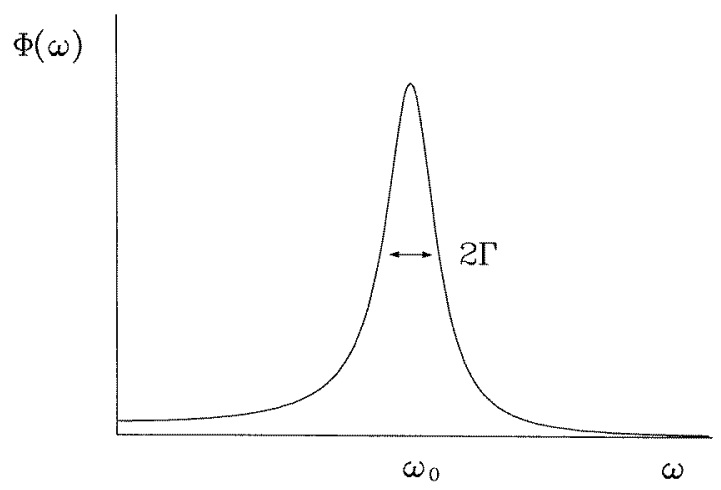

Figure 46. A typical power spectral density $\Phi(\omega)$ for quasimonochromatic noise $(\mathrm{QMN})$, as given by (117). 


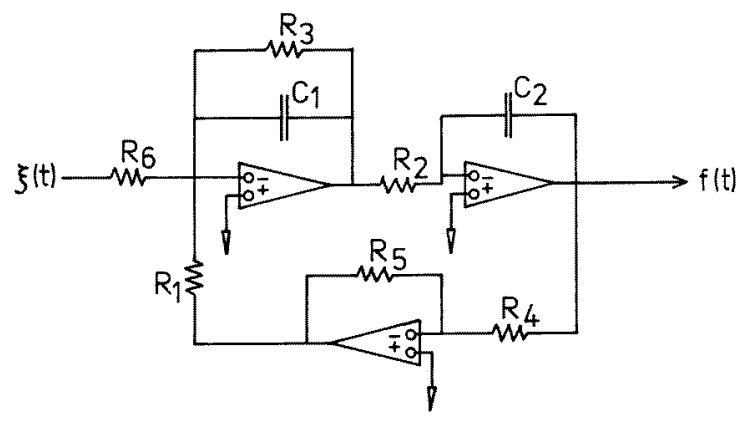

Figure 47. A block diagram of the underdamped harmonic oscillator circuit used to convert white noise $\xi(t)$ at the input into QMN $f(t)$ at the output (Dykman et al 1993e).

resistances $R_{1}-R_{3}$ as

$$
\omega_{0}^{2}=R_{2} / R_{1} \quad 2 \Gamma=R_{2} / R_{3}
$$

but, for $\Gamma<0.1$, were in practice determined (Dykman et al 1993e) from direct measurements of the mean-square velocity, using the fact that $\left\langle\dot{f}^{2}\right\rangle=D$, and by a resonance technique.

\subsection{Transitions in a bistable potential}

Fluctuational transitions between the stable states $x= \pm 1$ of the system (120) occur under QMN in a manner quite different from those under WN as discussed in section 3, where it was sufficient to reach the saddle point for the transition to happen with probability $\sim 1 / 2$. It was shown theoretically (Dykman 1990), and is also depicted in figure 48(a), that under QMN the excursions of the coordinate can go far beyond the potential barrier top (PBT) without the system leaving the potential well in which it started. This characteristic feature of QMN-driven systems was demonstrated (Dykman et al 1991b, 1993a) with an analogue electronic model of (120). Two samples of the fluctuating voltage in the circuit representing $x(t)$ in (120) are shown in figure 48(b). They correspond to fluctuations about the stable states $x_{1}=-1$ and $x_{2}=1$ and each exhibits occasional large fluctuations. It is apparent that in the course of such fluctuations $x(t)$ crosses the boundary point $x_{\text {top }}=0$ several times forwards and backwards, and then goes back to the initially occupied state without making a transition. Paths culminating in transitions to the other state are very much less frequent.

This behaviour can be understood intuitively (Dykman 1990) by noticing that, for $\omega_{0} \gg \Gamma$, the coordinate $x(t)$ mostly performs fast random oscillations at the frequency $\omega_{0}$, with an amplitude that varies relatively slowly, over the time $\sim \Gamma^{-1}$. However, for a transition to occur, it is necessary for the centre of oscillations to leave the first potential well and cross the central potential maximum; it is not sufficient just for $x(t)$ to do so. The picture becomes particularly simple provided the characteristic time scales of the motion are widely separated

$$
\Gamma \ll t_{\mathrm{r}}^{-1} \ll \omega_{0}
$$

where $t_{\mathrm{r}}$ is the relaxation time of the system $\left(t_{\mathrm{r}}=\frac{1}{2}\right.$ in the case of (120)). Writing the time-dependent coordinate of the system as a sum of the fast oscillating (at frequency $\omega_{0}$ ) and smooth parts,

$$
x=x_{c}(t)+x_{+}(t) \mathrm{e}^{\mathrm{i} \omega_{0} t}+x_{-}(t) \mathrm{e}^{-\mathrm{i} \omega_{0} t}
$$



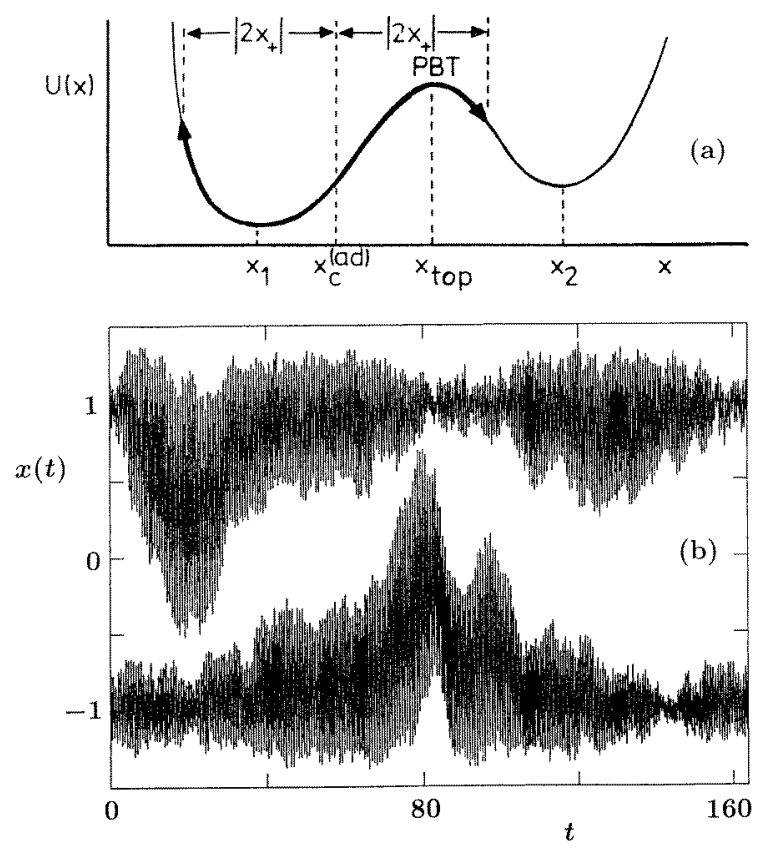

Figure 48. (a) Sketch of QMN dynamics in a general bistable potential $U(x)$. The coordinate oscillates (arrowed bold curve) with amplitude $2 x_{+}$about a centre of motion $x_{\mathrm{c}}^{(\mathrm{ad})}$, and can pass the potential barrier top (PBT) on each cycle without making a transition out of the initially occupied potential well. (b) Two samples of $x(t)$ measured with $D=192$ for the analogue electronic circuit model of (117), (118) and (120), exhibiting an example of an occasional large fluctuation from each of the attractors that fails to produce an inter-well transition (Dykman et al 1991b).

one can deduce that, in the spirit of the averaging method of Arnol'd (1978), the motion of the centre of oscillations $x_{\mathrm{c}}(t)$ may be regarded as occurring within a smoothed potential

$V\left(x_{\mathrm{c}}, x_{+}, x_{-}\right) \equiv \frac{1}{2 \pi} \int_{0}^{2 \pi} \mathrm{d} \psi U\left(x_{\mathrm{c}}+x_{+} \mathrm{e}^{\mathrm{i} \psi}+x_{-} \mathrm{e}^{-\mathrm{i} \psi}\right) \approx \frac{\omega_{0}}{2 \pi} \int_{0}^{2 \pi / \omega_{0}} U(x(t)) \mathrm{d} t$.

For the quartic potential (120) the smoothed potential (125) is given by the expression

$$
V\left(x_{\mathrm{c}}, x_{+}, x_{-}\right)=-\frac{1}{2} x_{\mathrm{c}}^{2}+\frac{1}{4} x_{\mathrm{c}}^{4}+3 x_{\mathrm{c}}^{2} x_{+}^{2}
$$

plus terms independent of $x_{\mathrm{c}}$.

The amplitude of the oscillations (i.e. the amplitude of the random force $f(t)$ ) varies over the time $\sim \Gamma^{-1}$. Therefore, if the relaxation time of the system is much less than $\Gamma^{-1}$, the centre of oscillations will follow variations in the amplitude of the oscillations adiabatically, so that it always remains at the minimum of the effective potential $V$ (125),

$$
\frac{\partial V}{\partial x_{\mathrm{c}}}=0 .
$$

The full curves of figure 49 show how $V$ varies with $x_{\mathrm{c}}$ for five values of $x_{+}$. The heavydashed curve represents the equilibrium value of $x_{\mathrm{c}}$ as given by (127), and the light-dashed curves represent the extreme limits of oscillation $x_{\mathrm{c}}-2 x_{+}, x_{\mathrm{c}}+2 x_{+}$.

It is interesting to note that, as the amplitude of the oscillations increases from zero, the lower limit $x_{\mathrm{c}}-2 x_{+}$moves at first towards lower $x$ but then, at $x=-\sqrt{5 / 3} \approx-1.29$, 


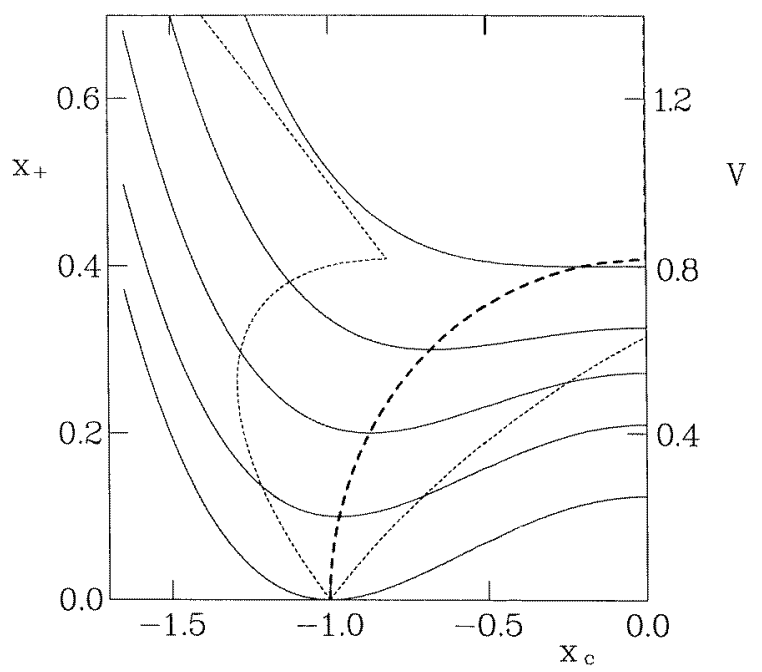

Figure 49. The smoothed potential $V\left(x_{\mathrm{c}}\right)$ (full curves) given by (125) for the system (117)-(120) for the five different oscillation amplitudes $x_{+}$, reading from bottom to top: $0,0.1,0.2,0.3$, 0.4 . For clarity, the curves have been shifted up by the amounts indicated by their intersections with the right-hand ordinate axis at $x_{\mathrm{c}}=0$. The heavy-dashed curve shows the evolution of $x_{\mathrm{c}}$, plotted horizontally, as a function of $x_{+}$. The extreme limits of oscillation $x_{\mathrm{c}}-2 x_{+}, x_{\mathrm{c}}+2 x_{+}$ are shown by the light-dashed curves (Dykman et al 1995f).

turns back towards the positive $x$-direction. One would expect this feature (Dykman 1990) to lead to the appearance of a singular point in the stationary probability distribution $P(x)$, a prediction to which we will return in section 6.3.

Mean escape rates $T$ between the two potential wells have been measured (Dykman et al 1993e) for the QMN-driven model, yielding the results shown in figure 50. They are plotted as $\ln T$ against $D^{-1}$ for comparison with the theoretical expression

$$
T \propto \exp \left(R_{\mathrm{it}} / D\right)
$$

with $R_{\mathrm{it}}=\omega_{0}^{2} / 3 \Gamma$ for (120) (Dykman 1990). The analogue data (pluses) lie on a straight line to an excellent approximation. For the two sets of parameters used, the gradients were found to be: $R_{\mathrm{it}}=1600$ for $\omega_{0}=9.81, \Gamma=0.021$; and $R_{\mathrm{it}}=719$ for $\omega_{0}=9.95$, $\Gamma=0.045$. These results are to be compared with the theoretical predictions of 1528 and 733 , respectively, with which they agree within experimental error. The results were also confirmed by digital simulations (crosses). It is interesting to note that $R_{\mathrm{it}}$ is substantially larger than the activation energy required to reach the PBT: the probability of reaching the PBT exceeded the transition probability by a factor of approximately 100, thereby emphasizing one of the major differences between QMN and WN, for which the factor would be two.

\subsection{The stationary distribution}

From the picture of random motion of the QMN-driven system as fast oscillations superimposed on the slow drift of their centre (cf figure 49), it is possible to infer the form of the probability distribution. At a given $x$, this distribution is determined, to logarithmic accuracy, by the probability density for an oscillating coordinate to reach this $x$ as the amplitude of fluctuational vibrations increases. For very small fluctuations (compared to 

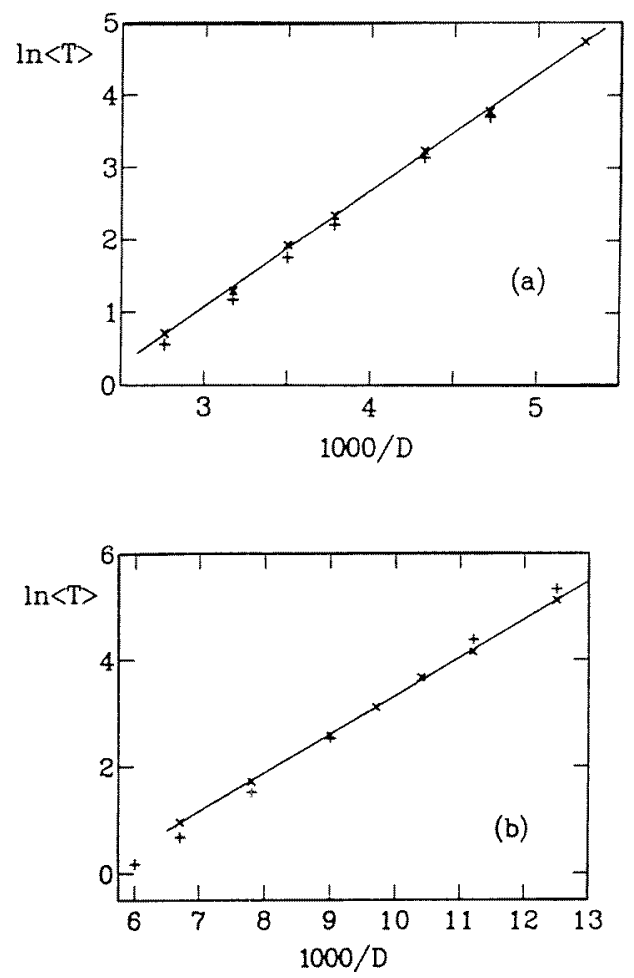

Figure 50. Dependence of the mean escape time $\langle T\rangle$ on the noise strength $D$, plotted for comparison with (128). The pluses and crosses represent analogue and digital simulations, respectively, for: (a) $\omega_{0}=9.81, \Gamma=0.021$; (b) $\omega_{0}=9.95, \Gamma=0.045$. The straight lines are fits to the analogue data (Dykman et al 1993e).

the distance to the saddle point), the extremes of the motion $x(t)$ (light-dashed curve) are approximately symmetrical about the stable state at $x=-1$ (where we assume the system to be localized initially). As the fluctuation amplitude increases, however, the hardening of the potential $V$ for the centre of oscillations for $x_{\mathrm{c}}<-1$, and its softening for $x_{\mathrm{c}}>-1$, cause $\left|x_{\mathrm{c}}\right|$ to decrease. Eventually, the latter effect dominates the evolution of the lower extreme $-2\left|x_{+}\right|+x_{\mathrm{c}}$ of the oscillating $x(t)$ and this extreme passes its limiting value and starts to increase. With further increase in the oscillation amplitude, the minimum in $V\left(x_{\mathrm{c}}\right)$ becomes shallower and moves towards $x_{\mathrm{c}}=0$. For the oscillation amplitude $2\left|x_{+}\right|=(2 / 3)^{1 / 2}$ this minimum reaches $x_{\mathrm{c}}=0$ and sticks there (and so does $x_{\mathrm{c}}$ ), so that the motion with higher amplitudes just consists of vibrations symmetrically distributed about $x_{\mathrm{c}}=0$. The extremes of the motion will increase equally towards positive and negative $x$ with increasing fluctuation amplitude, as shown in the latter case by the abrupt change in slope of the lightdashed curve on the left of figure 49. One would expect to see a corresponding change in the gradient of the logarithm of the probability distribution $P(x)$ at the point where reaching $x$ via oscillations about $x_{\mathrm{c}} \approx-1$ is replaced by that via oscillations about $x_{\mathrm{c}}=0$.

Without going into the full quantitative theory of the shape of the probability distribution we can put these ideas onto a more quantitative basis by noticing that, according to equation (118), the distribution of the amplitude $2\left|f_{+}\right|$of the vibrations of the force 


$$
\begin{aligned}
f(t) \approx f_{+}(t) \exp \left(\mathrm{i} \omega_{0} t\right) & +\mathrm{CC} \text { is Gaussian, } \\
P_{f}\left(\left|f_{+}\right|\right) & \approx \text { constant } \times \exp \left(-2 \omega_{0}^{2}\left|f_{+}\right|^{2} / D\right) .
\end{aligned}
$$

On the other hand, the amplitude of the fast oscillations of the coordinate $x_{+}$is related to $f_{+}$ just by the expression $x_{+}=f_{+} / \mathrm{i} \omega_{0}$, which immediately follows from the equation of motion with account taken of the fact that the frequency $\omega_{0}$ exceeds all dynamical frequencies of the system. Therefore, the distribution over $x_{+}$has a form

$$
P\left(\left|x_{+}\right|\right) \propto \exp \left(-2 \omega_{0}^{4}\left|x_{+}\right|^{2} / D\right) .
$$

When the system reaches the point $x$, which for definiteness we consider to be the left-hand limit of motion in the left-hand potential well, then

$$
x=x_{\mathrm{c}}-2 x_{+} .
$$

The position of the centre of oscillations is related to the amplitude $\left|x_{+}\right|$by the adiabatic condition (127), giving

$$
x_{\mathrm{c}}\left(-1+x_{\mathrm{c}}^{2}+6 x_{+}^{2}\right)=0
$$

which has three real solutions for $x_{\mathrm{c}}$ when $\left(1-6 x_{+}^{2}\right)>0$. The range of validity of each solution is determined by demanding that the stationary point of the smoothed potential (126) be a minimum, $\partial^{2} V / \partial x_{\mathrm{c}}^{2}>0$. Thus, we obtain

$$
x_{\mathrm{c}}= \begin{cases}-\sqrt{1-6 x_{+}^{2}} & \left(x_{+}<\sqrt{1 / 6}\right) \\ 0 & \left(x_{+}>\sqrt{1 / 6}\right)\end{cases}
$$

as the explicit expression for the heavy-dashed curve in figure 49. It is convenient to recast (131) and (133) as expressions for the amplitude $x_{+}$in terms of $x$ :

$$
x_{+}= \begin{cases}-\frac{1}{10}\left(2 x+\sqrt{10-6 x^{2}}\right) & \left(x_{+}<\sqrt{1 / 6}\right) \\ -\frac{1}{2} x & \left(x_{+}>\sqrt{1 / 6}\right) .\end{cases}
$$

Equations (130) and (134) provide the explicit form of the probability distribution over $x$ (Dykman 1990). Clearly, this distribution displays a discontinuity at $x=-\sqrt{5 / 3}$, which is the analogue of the caustic-related discontinuities in white-noise-driven systems discussed in section 3. The related switching of optimal paths is discussed in section 6.4.

The non-analytic behaviour of the probability distribution was obtained in the doubleadiabatic approximation where the vibrations of the system follow adiabatically the vibrations of the random force, and the position of the centre of oscillations follows adiabatically the instantaneous value of the vibration amplitude. If the latter approximation is weakened and non-adiabatic corrections in the parameter $\Gamma t_{\mathrm{r}}$ are taken into account, the square-root singularity of the logarithm of the probability distribution is replaced by a steep function, which was obtained by Dykman et al $(1991 \mathrm{~b}, 1993 \mathrm{e})$ to the lowest order in $\Gamma t_{\mathrm{r}}$ and is shown by curve (c) in figure 51 .

Some analogue experimental results are plotted for comparison with theory in figure 51 . Curve (a) represents the simplest version of the theory, leading to the singularity in $\ln P(x)$ discussed above. Curve (b) shows the experimental measurements showing that indeed there is no singularity but, rather, a region where $\ln P(x)$ is extremely steep compared to the equivalent results for driving by WN (Dykman et al 1993e). These data are in good, although not perfect, agreement with curve (c) representing the non-adiabatic theory. For larger negative values of $x$, the gradient of $|\ln P|$ evidently decreases again. The crossover from larger to smaller slope of $|\ln P|$ occurs in a narrow region around a switching point 


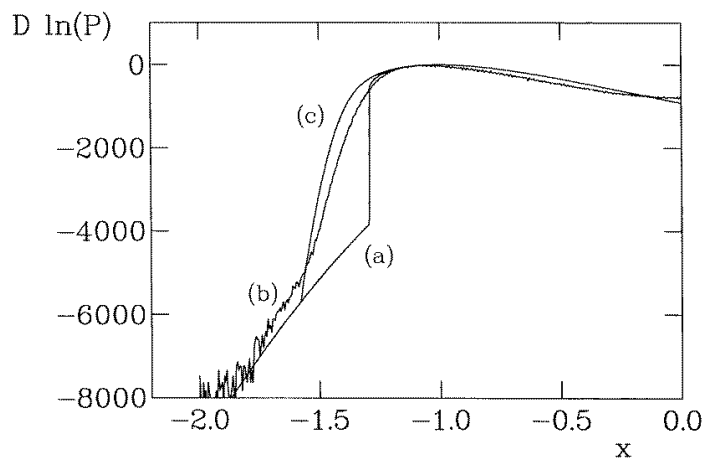

Figure 51. The probability distribution $P(x)$ for the system (117)-(120), plotted in the form $D \ln (P)$ where $D$ is the noise intensity, as a function of negative $x$. Curve (a) is the adiabatic theory; the jagged curve (b) represents experimental measurements on an analogue electronic model of (117)-(120); and curve (c) shows the theory corrected to first order in non-adiabatic effects. The parameters in all cases were $D=700, \omega_{0}=9.81$ and $\Gamma=0.021$ (Dykman et al 1995f).

$x_{\mathrm{sw}}$ which may be estimated from the experimental data as $x_{\mathrm{sw}} \approx-1.5$. It is naturally associated with the fact that for $|x|>\left|x_{\mathrm{sw}}\right|$ the distribution is formed by fluctuations that first drive $x_{\mathrm{c}}$ to the point $x_{\mathrm{c}}=0$ and then continue to increase in the amplitude so that eventually a given point $x$ is encountered by fluctuational vibrations about $x_{\mathrm{c}}=0$ (Dykman 1990). The dependence of $\ln P$ on $x$ in this range is given by equations (130) and (134).

\subsection{Large fluctuations and observation of a switching point}

As discussed above in section 3, a powerful method of investigating large occasional fluctuations is to examine their prehistory by building the probability distribution of paths ending at the point of interest (Dykman et al 1992c). In applying this technique, we ask the question: if a rare fluctuation carries the system to a final point $x_{f}$ at a time $t_{f}$, what is the probability that it passed through a point $x$ at time $t$ ? The answer is given by the prehistory probability distribution $p_{h}\left(x, t ; x_{f}, t_{f}\right)$; usually, for convenience, we set $t_{f}=0$ as before.

The method used for the measurement of $p_{h}\left(x, t ; x_{f}, 0\right)$ in an experimental system fluctuating under WN was described in section 3. However, as we mentioned, the original theoretical formulation of the prehistory problem (Dykman et al 1992c) applies irrespective of the type of noise. In the case of QMN we are primarily interested in the motion of the centre of oscillations $x_{\mathrm{c}}$, rather than in the oscillations themselves, and therefore it is particularly convenient to adopt an approach in which the prehistory of $x_{\mathrm{c}}$ is followed. Respectively, successive realizations of $x(t)$ are examined until one is found that arrives at $x_{f}$. Taking this point as the zero of time $\left(t_{f}=0\right)$ and working backwards, the path of the centre of oscillations leading to the final point is recorded and added to the distribution. The process then repeats, building up a prehistory distribution $P_{h}\left(x_{\mathrm{c}}, t ; x_{f}, t\right)$ of $x_{\mathrm{c}}$ for that value of $x_{f}$.

For values of $x_{f}$ lying well away from the switching point $x_{\mathrm{sw}}$ the dependence of $P_{h}\left(x_{\mathrm{c}}, t ; x_{f}, 0\right)$ on $x_{f}$ is smooth: a small variation of $x_{f}$ gives rise to a small change of the optimal path of the centre of oscillations $x_{\mathrm{c}}(t)$ along which the system arrives at $x_{f}$, 
as the centre of oscillations remains close to the corresponding stable state $x_{\mathrm{st}}= \pm 1$. One would expect that this might no longer be true for the points $x_{f}$ lying on the other sides of $x_{\mathrm{sw}}$, because the corresponding paths should be topologically different: the points with $\left|x_{f}\right|>\left|x_{\mathrm{sw}}\right|$ are reached along paths where the centre of oscillations $x_{\mathrm{c}}$ is brought first to the point $x_{\mathrm{c}}=0$ and then sticks to it while the amplitude of the vibrations about $x_{\mathrm{c}}$ increases and eventually becomes equal to $\left|x_{f}\right|$. The width of the range of $x_{f}$ where the switching occurs between the two types of path is proportional to the noise intensity and is therefore expected to be small.

These theoretical arguments have been confirmed (Dykman et al 1995f) through analogue electronic experiments. Some typical measurements of the prehistory probability density $P_{h}\left(x_{\mathrm{c}}, t ; x_{f}, 0\right)$ for $x_{f}$ close to the experimentally found value $x_{\mathrm{sw}} \approx-1.5$ are plotted in figure 52. For $x_{f}=-1.55$ (figure 52(c)) the position of the centre of oscillations at the instant $t=0$ of arrival at $x_{f}$, and for some time before this arrival, lies with very high probability at $x_{\mathrm{c}}=0$, in agreement with what has been said above. It can be seen from figures 52(b) and (a), however, that the $t=0$ peak corresponding to $x_{\mathrm{c}}$ lying close to the stable position $x_{\mathrm{st}}=-1$ is growing rapidly with decreasing $\left|x_{f}\right|$. There must clearly be a critical value of $x_{f}$ (evidently of magnitude slightly smaller than 1.5-this is in agreement with the data in figure 51 where the change in slope occurs for $|x|$ slightly less than 1.5), which we may identify with the switching point $x_{\mathrm{sw}}$, at which the integrated intensities of the two $t=0$ peaks at $x_{\mathrm{c}} \simeq x_{\mathrm{st}}=-1$ and at $x_{\mathrm{c}}=0$ will be equal. As $x_{f}$ moves away from $x_{\mathrm{sw}}$, the relative intensities of these peaks, and correspondingly the relative probabilities of the two types of path, vary very rapidly as discussed above.

Yet another interesting feature of the prehistory probability density seen from figure 52 concerns the behaviour of $P_{h}\left(x_{\mathrm{c}}, t ; x_{f}, 0\right)$ for large $|t|$. The theoretical formulation of the prehistory problem (Dykman et al 1992c) referred to the case where the probability of reaching a small vicinity of the given state from the corresponding metastable state was larger than the probability of switching between the states. Therefore, when the trajectory of the system was traced back in time it would approach, over the relaxation time or the correlation time of noise $\left(\Gamma^{-1}\right.$, in the present case), whichever is larger, the vicinity of the metastable state. However, if the trajectory is traced further backward in time, up to the times exceeding the reciprocal rates of interstate transitions, one would find that eventually the prehistory probability density goes over into the full stationary distribution of the system, rather than the quasistationary distribution about the appropriate metastable state. This is precisely what happens in the system under consideration for $\left|x_{f}\right|<\left|x_{\mathrm{sw}}\right|$.

The situation is different for $\left|x_{f}\right|>\left|x_{\mathrm{sw}}\right|$. In this case the probability of reaching a given $x_{f}$ is less than the probability of transitions between the stable states (Dykman 1990). Therefore, prior to reaching a point $x_{f}$ the system, whichever stable state it occupied initially, will have become equally distributed over the both states, and the fluctuation that brought it to $x_{f}$ might have started in either of these states. This is, of course, a consequence of the symmetry. However, it is clear that the prehistory probability density $P_{h}\left(x_{\mathrm{c}}, t ; x_{f}, 0\right)$ should immediately start splitting with increasing $|t|$ into two peaks that correspond to small-amplitude fluctuations about the stable states, as observed (instead of remaining a single peak over the time $\sim \Gamma^{-1}$ and then splitting into the two peaks due to the inter-well transitions). There is also a small central peak at $x_{\mathrm{c}}=0$ seen in figures 51(a)-(c) which reflects a comparatively large probability of fluctuations bringing the centre of oscillations to $x=0$; this peak arises from the competition between a large prefactor and a small exponential factor in the occupation probability of the state, and it diminishes rapidly with decreasing noise intensity. 


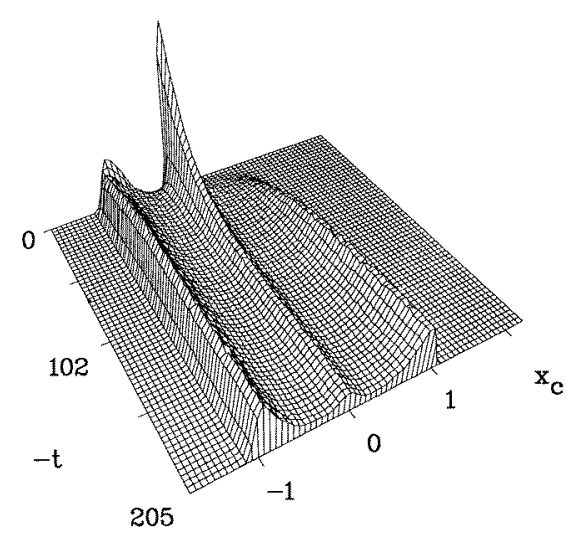

(a)

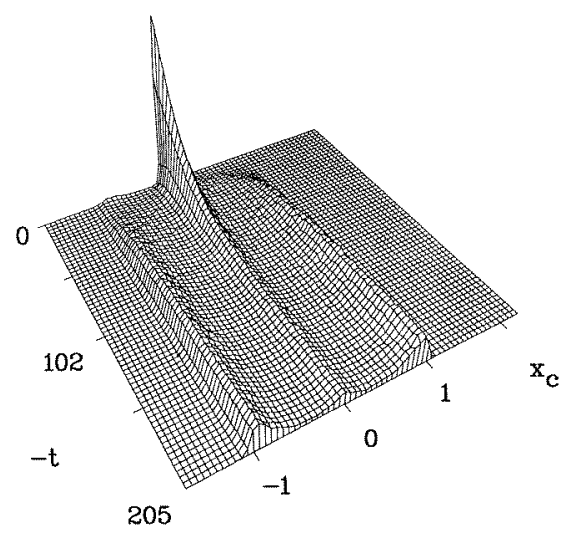

(c)

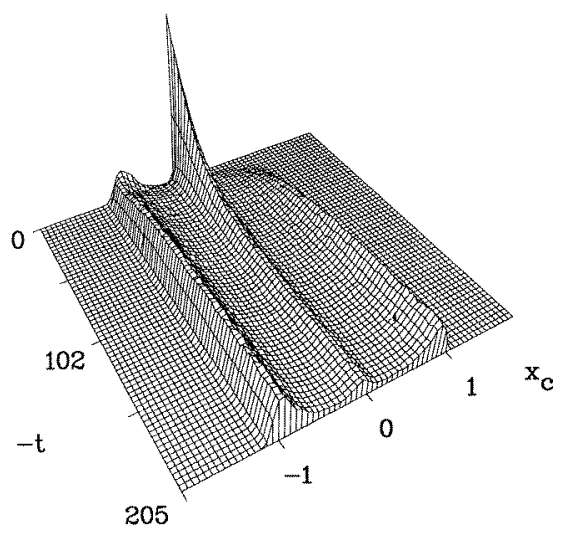

(b)

Figure 52. The prehistory probability distribution $P_{h}\left(x_{\mathrm{c}}, t ; x_{f}, 0\right)$ of the centre of oscillations $x_{\mathrm{c}}$ for arrivals at the final points: (a) $x_{f}=-1.5$; (b) $x_{f}=-1.52$; (c) $x_{f}=-1.55$ (Dykman et al 1995f).

We note that, historically, these theoretical and experimental results on QMN-driven systems preceded the extensive results on switching between optimal fluctuational paths for various types of white-noise-driven systems discussed in detail in section 3 .

\section{Deterministic nonlinear phenomena}

The phenomena discussed above have all been stochastic in character and, arguably, it is under these conditions that the analogue technique is at its most effective and powerful. One reason is that extraneous noise generated by active components such as multipliers or operational amplifiers usually has an insignificant effect compared to the external noise applied to the model (although there are exceptions, cf Mannella et al 1986). Analogue models have also, however, been used to investigate a wide range of deterministic phenomena, including a pioneering investigation of human heart rate variability (Van der Pol and Van der Mark 1928), hopping between strange attractors as a possible explanation of $1 / f$ 
noise (Arecchi and Lisi 1982), chaos in driven nonlinear oscillators (Holmes 1979, Linsay 1981, Testa et al 1982) and in the Lorenz system (Robinson 1990), multiperiodic orbits in a pendulum with a vertically oscillating pivot (Smith and Blackburn 1994), synchronized chaos in coupled oscillators (Heagy et al 1994), modulation-induced negative differential resistance (Dow et al 1987, Gwaki et al 1993) and zero-dispersion nonlinear resonance (Luchinsky et al 1996a, 1997). We now consider the latter two cases, as examples.

\subsection{Modulation-induced negative differential resistance}

Modulation-induced negative differential resistance (MINDR) was first observed, by chance, in an analogue electronic model; only later was it explained and discussed theoretically (Dow et al 1987). It arises in damped bistable systems when driven by a multiplicative periodic force: a parameter regime exists within which the response amplitude decreases with increasing drive amplitude, and it is this counter-intuitive effect that constitutes MINDR. The phenomenon is at its most pronounced in overdamped, deterministic, bistable systems, but it is also robust in the presence of moderate levels of noise and inertia (Gwaki et al 1993). MINDR was discovered in the cubic bistable system

$$
\dot{x}=-x^{3}+\lambda(t) x^{2}-Q x+R
$$

with the constants $Q=3$ and $R=0.7$. The periodically varying parameter $\lambda(t)=\lambda(t+\tau)$ is conveniently written in the form

$$
\lambda(t)=\lambda_{0}+V_{0} h(t)
$$

where $h(t)$ is a normalized modulation of zero mean, satisfying

$$
\tau^{-1} \int_{0}^{\tau} \mathrm{d} t|h(t)|=1 \quad \int_{0}^{\tau} \mathrm{d} t h(t)=0 .
$$

The system (135) is a particular example of the more general system

$$
\dot{x}=f(x)+V_{0} h(t) g(x)
$$

for which, in the present case,

$$
f(x)=-x^{3}+\lambda_{0} x^{2}-Q x+R \quad g(x)=x^{2} .
$$

For fixed values of $\lambda_{0}$ and $V_{0}$, we define the response amplitude $A\left(\lambda_{0}, V_{0}\right)$ as the difference between the extreme values $x_{+}$and $x_{-}$of $x(t)$. The squares in figure 53(a) represent data from an electronic model with $\lambda_{0}=3.6 \pm 0.1$. They show how the response amplitude $A$ varies with the drive amplitude $V_{0}$ of a square-wave modulation with fixed period $\tau=1 / 6$, such that

$$
\begin{array}{ll}
h(t)=+1 & \text { for } 0 \leqslant t \leqslant \tau / 2 \\
h(t)=-1 & \text { for } \tau / 2 \leqslant t \leqslant \tau .
\end{array}
$$

The squares in figure 53(b) show how the extremes $x_{ \pm}$of the response vary with drive amplitude. These experimental data of figure 53 reveal immediately that there is a range of negative differential resistance where $\mathrm{d} A / \mathrm{d} V_{0}<0$. The bold and thin curves in each case represent numerical solutions of the equations

$$
\begin{aligned}
& \frac{1}{2} \tau=\int_{x_{-}}^{x_{+}} \mathrm{d} x\left[f(x)+V_{0} g(x)\right]^{-1} \\
& \frac{1}{2} \tau=\int_{x_{+}}^{x_{-}} \mathrm{d} x\left[f(x)-V_{0} g(x)\right]^{-1}
\end{aligned}
$$




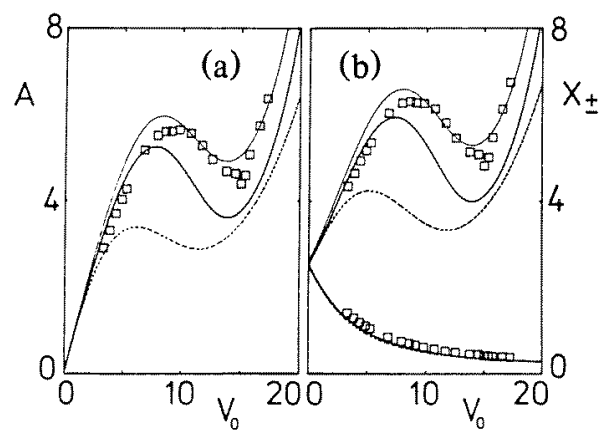

Figure 53. (a) Response amplitude $A=\left|x_{+}-x_{-}\right|$of the cubic bistable system (135) and (136) as a function of drive amplitude $V_{0}$. The data are measurements from an analogue electronic model with $\lambda_{0}=3.6 \pm 0.1$, and the lower and upper full curves represent theory for $\lambda_{0}=3.60$ and 3.65 , respectively. The dashed curve shows the corresponding behaviour calculated for a sine wave with $\lambda_{0}=3.6$. In each case, the regions of negative slope correspond to the occurrence of MINDR. (b) Curves showing the corresponding values of the limits $x_{ \pm}$(Dow et al 1987).

for $V_{0}=3.60$ and $V_{0}=3.65$, respectively. They are clearly in good agreement with the experimental data. The dashed curves in figures 53(a) and (b) show what happens when the square-wave modulation is replaced with a sine wave: MINDR is again evident, but the effect is weaker.

MINDR has been analysed in detail by Lambert (Dow et al 1987) in the extreme limit of large-amplitude, high-frequency, modulation, such that

$$
V_{0} \rightarrow \infty \quad \tau \rightarrow 0 \quad \frac{1}{2} V_{0} \tau=\mu
$$

where $\mu$ is finite. In this limit, for square-wave modulation, equations (141) and (143) reduce to

$$
\begin{aligned}
& \int_{x_{-}}^{x_{+}} \frac{\mathrm{d} x f(x)}{[g(x)]^{2}}=0 \\
& \int_{x_{-}}^{x_{+}} \frac{\mathrm{d} x}{g(x)}=\mu .
\end{aligned}
$$

For the electronic circuit model, with $f(x)$ and $g(x)$ given by (139), we obtain

$$
\begin{gathered}
-\ln \left(x_{+} / x_{-}\right)+\lambda_{0}\left(x_{-}^{-1}-x_{+}^{-1}\right)-\frac{1}{2} Q\left(x_{-}^{-2}-x_{+}^{-2}\right)+\frac{1}{3} R\left(x_{-}^{-3}-x_{+}^{-3}\right)=0 \\
x_{-}^{-1}-x_{+}^{-1}=\mu .
\end{gathered}
$$

It can thus be seen that, for large $V_{0}$, the bounds $x_{ \pm}$and hence also the response amplitude $A$ approach universal functions of the parameter $\mu$, which are independent of $\tau$ and $V_{0}$ separately. In this limit, $\tau$ is effectively just a scaling factor for the drive amplitude $V_{0}$.

These universal functions have been calculated (Dow et al 1987). The results were generalized to describe the response to a periodic force of arbitrary form through the introduction as a coefficient of castellation $\epsilon$ that gives a measure of the departure from a square-wave shape: for a square wave, $\epsilon=0$. MINDR is difficult to treat analytically in the presence of noise, or where there is an inertial term in the equation of motion. Digital techniques have been used, however, to show that the phenomenon persists in the presence 
of moderate inertia and noise, but that it can be destroyed if they become too large (Gwaki et al 1993).

\subsection{Zero-dispersion nonlinear resonance}

Zero-dispersion nonlinear resonance (ZDNR) is an interesting phenomenon that can occur in a periodically-driven underdamped oscillator whose eigenfrequency (without the driving force, and in the absence of damping) possesses one or more extrema as a function of energy, i.e. the same kind of oscillator that can exhibit noise-induced spectral narrowing (Dykman et al 1990b), zero-dispersion peaks (Soskin 1989, 1992, Stocks et al 1993c) and zero-dispersion stochastic resonance (Stocks et al 1992, and see section 4.4). Many real physical systems including, for example, SQUIDs (Barone and Paterno 1982, Kautz 1996), relativistic oscillators (Kim and Lee 1995, Luchinsky et al 1996b), electrical circuits (Hayashi 1964), and polymeric molecules, are of this type. ZDNR was discovered theoretically by Soskin (1994) for a zero-dispersion oscillator in the absence of damping, and was subsequently observed experimentally and described theoretically for a weakly dissipative system (Luchinsky et al 1996a). The status of research on ZDNR has been summarized by Soskin et al (1997). The phenomenon has some features that, at first sight, are rather surprising. For example, there can be a strong response to the external driving force even in the absence of matching between the driving frequency and any eigenfrequency of the (undriven, undamped) system. An analogue electronic model has been used both to demonstrate and investigate the phenomenon. In particular, its bifurcation diagram in the plane of the driving force parameters has been constructed by locating the transition lines separating different regimes of behaviour.

To understand the physical origins of ZDNR, we take as an example a one-dimensional potential system subject to a weak linear friction and a weak periodic force, such that

$$
\dot{x}=p \quad \dot{p}=-\frac{\mathrm{d} U(x)}{\mathrm{d} x}-\Gamma p+h \cos \left(\omega_{f} t\right) .
$$

Our goals will be to find period-1 orbits and to describe the transition regimes. (Note that higher-order resonances can be treated in a similar way.) With these aims, we transform to the slow variables action $I$ and phase difference $\tilde{\psi}=\psi-\omega_{f} t$ between the force and the response; we neglect high-frequency oscillatory terms. Then (146) can be reduced to

$$
\dot{I}=-h x_{1} \sin (\tilde{\psi})-\Gamma I \quad \dot{\tilde{\psi}}=\omega-\omega_{f}-\frac{\mathrm{d} x_{1}}{\mathrm{~d} I} h \cos (\tilde{\psi})
$$

where $\omega \equiv \omega(I)$ is the eigenfrequency corresponding to $I$, and $x_{1}$ is the first harmonic in the Fourier expansion of $x$ :

$$
x \equiv q(I, \psi)=2 \sum_{n=0}^{\infty} x_{n}(I) \cos (n \psi) .
$$

The period-1 orbits are located by finding the stationary solutions of (147) with non-zero action. These can be of two types

$$
\begin{aligned}
& \tilde{\psi}_{\mathrm{st}}=-\arcsin \left\{\Gamma I_{\mathrm{st}} /\left[h x_{1}\left(I_{\mathrm{st}}\right)\right]\right\} \\
& \tilde{\psi}_{\mathrm{st}}=\arcsin \left\{\Gamma I_{\mathrm{st}} /\left[h x_{1}\left(I_{\mathrm{st}}\right)\right]\right\}-\pi
\end{aligned}
$$

where $I_{\mathrm{st}}$ satisfies the equation

$$
\omega\left(I_{\mathrm{st}}\right)-\omega_{f}= \pm h \frac{\mathrm{d} x_{1}\left(I_{\mathrm{st}}\right)}{\mathrm{d} I_{\mathrm{st}}}\left(1-\left(\frac{\Gamma I_{\mathrm{st}}}{h x_{1}\left(I_{\mathrm{st}}\right)}\right)^{2}\right)^{1 / 2}
$$




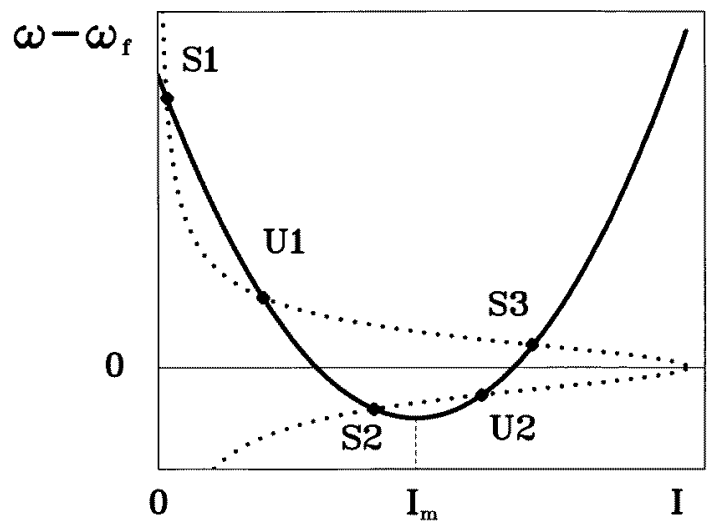

Figure 54. The typical dependence of $\left(\omega-\omega_{f}\right)$ on the action $I$ for a system with a minimum in $\omega(E)$ (full curve), and solutions of (149) for stationary actions. The dotted curve represents $\pm h x_{1}^{\prime}\left[1-\left(\Gamma I / h x_{1}\right)^{2}\right]^{1 / 2}$. The intersections corresponding to stable points (attractors) and unstable ones (saddles) are labelled S1-S3 and U1 and U2, respectively (Luchinsky et al 1996a).

in which the plus and minus refer to (149) and (150), respectively. Equation (151) can be solved explicitly for very small $h$, or numerically for larger $h$ : see figure 54. It is clear from inspection that, just as in the dissipationless case (Soskin 1994), there will be a range of $\omega_{f}$ for which the extremum of the full curve comes above the abscissa $\left(\omega=\omega_{f}\right)$ line but still intersects the upper dashed curve near $I_{m}$, i.e. a large-amplitude response can exist even if $\omega_{f}$ is beyond the spectrum of eigenfrequencies.

For further analysis, we turn to an example (Dykman et al 1990b) of a simple quartic single-well potential whose dependence of eigenfrequency on energy possesses an extremum,

$$
U(x)=\frac{1}{2} \omega_{0}^{2} x^{2}+\frac{1}{3} \beta x^{3}+\frac{1}{4} \gamma x^{4} \quad \frac{9}{10}<\frac{\beta^{2}}{\gamma \omega_{0}^{2}}<4 .
$$

Note that, although the $U(x)$ considered by Dykman et al (1990b) contained a linear term, it is reducible to (152) by a suitable change of variable. For (152) with $\omega_{0}=1, \beta=5 / 3$, $\gamma=1$, there is a minimum in $\omega(I): \omega_{\mathrm{m}}=0.805 ; I_{\mathrm{m}}=0.187 ; \omega_{\mathrm{m}}^{\prime \prime} \equiv \mathrm{d}^{2} \omega\left(I_{\mathrm{m}}\right) / \mathrm{d} I_{\mathrm{m}}^{2}=10.5$; $x_{1 \mathrm{~m}} \equiv x_{1}\left(I_{\mathrm{m}}\right)=0.325$. Figure 55 shows the bifurcation diagram in the plane of the driving force parameters for $\Gamma=0.011$. Its structure is typical of that expected for any system with an extremum in eigenfrequency as a function of energy (or action). The data points represent measurements on an analogue model of (146) and (152) of similar design to those already described. The theoretical curves were obtained from the condition that curves corresponding to the left-hand and right-hand sides of (149) touch rather than cross each other (cf figure 54). They are in good agreement with the experimental data. Within the region bounded by the full curves (except very close to the cusps of the full curves), point $\mathrm{S} 1$ in figure 54 is well separated in action from $\mathrm{S} 2 / \mathrm{S} 3$. The response corresponding to $\mathrm{S} 2 / \mathrm{S} 3$ is always strongly nonlinear. The response corresponding to $\mathrm{S} 1$ is linear in the region far below the upper full curve. It starts to be nonlinear when closer to the line but even then it is still significantly smaller than $\mathrm{S} 2 / \mathrm{S} 3$; in order to distinguish $\mathrm{S} 1$ from S2/S3, we shall refer to it as 'linear' within the whole region bounded by the full curves.

The evolution of the phase space of the slow parameters as $\omega_{f}$ increases for fixed $h$ is of particular interest, and has been calculated numerically as shown in figure 56. One can see 


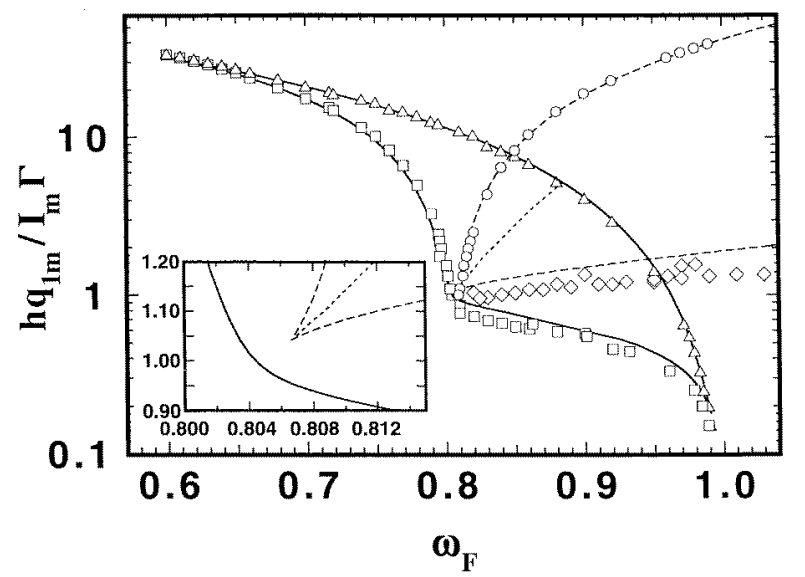

Figure 55. The bifurcation diagram in the plane of the driving force parameters for (146) and (152) with $\omega_{0}=\gamma=1.0, \beta=5 / 3, \Gamma=0.011$ (the normalization of the vertical axis is $\left.x_{1 \mathrm{~m}} /\left(\Gamma I_{\mathrm{m}}\right)=159.0\right)$. Full curves bound the region within which both the linear (S1) and one or both of the nonlinear (S2, S3) responses can exist: the upper curve (theory) and triangles (analogue electronic simulation) mark the boundary of the linear response, and the lower curve and squares mark that for the nonlinear responses. Dashed curves bound the region where both nonlinear responses (S2, S3) coexist: the upper dashed curve (theory) and circles (simulation) mark the boundary for the lower action attractor (S2), and the lower dashed curve and diamonds mark that for the larger action attractor (S3). The calculated ZDNR/NR transition is shown by the dotted curve. The inset provides an enlarged plot of the region near the cusp (Luchinsky et al 1996a).

a distinct difference in the structure of the BAs of the nonlinear responses at different $\omega_{f}$. At smaller $\omega_{f}$ (figures 56(a) and (b)), the phase difference between attractor and saddle (the outer saddle, in cases where the BA has two saddles) for each BA is negligible, whereas for larger $\omega_{f}$ (figures 56(c)-(e)) it is of the same order as the characteristic width of the BA. This holds true throughout the whole region enclosed by the full curves in figure 55 (except, very close to the bifurcation curves), the two types of behaviour being separated by the dotted curve. In analogy with the dissipationless case (Soskin 1994), the parameter ranges to the left and right of the dotted curve can be consistently defined as the zerodispersion (ZDNR) and conventional (NR) stages of nonlinear response (and the definition can be formulated in a similar way for the original system (146) in terms of a stroboscopic Poincaré section).

In the dissipationless case, the transition between the ZDNR and NR stages as parameters change occurs (Soskin and Luchinsky 1995) through separatrix reconnection (Howard and Hohs 1984), resulting in a different topology of separatrices between regions of trapped and untrapped motion: the separatrices are homoclinic or heteroclinic for ZDNR or NR, respectively (Soskin 1994, Soskin and Luchinsky 1995). In the presence of dissipation, the transition occurs typically via a saddle connection (Guckenheimer and Holmes 1983) as can be seen from figures 56(b) and (c). It also results in a change of topology of the BAs of the nonlinear responses. Just before the bifurcation, at the ZDNR stage, the basin of attraction (BA) corresponding to the larger action attractor (S3) encompasses the other one (S2), whereas the opposite applies for the NR stage just after the bifurcation: see figures 55(b) and (c). Note that the transition can also occur continuously via a path 

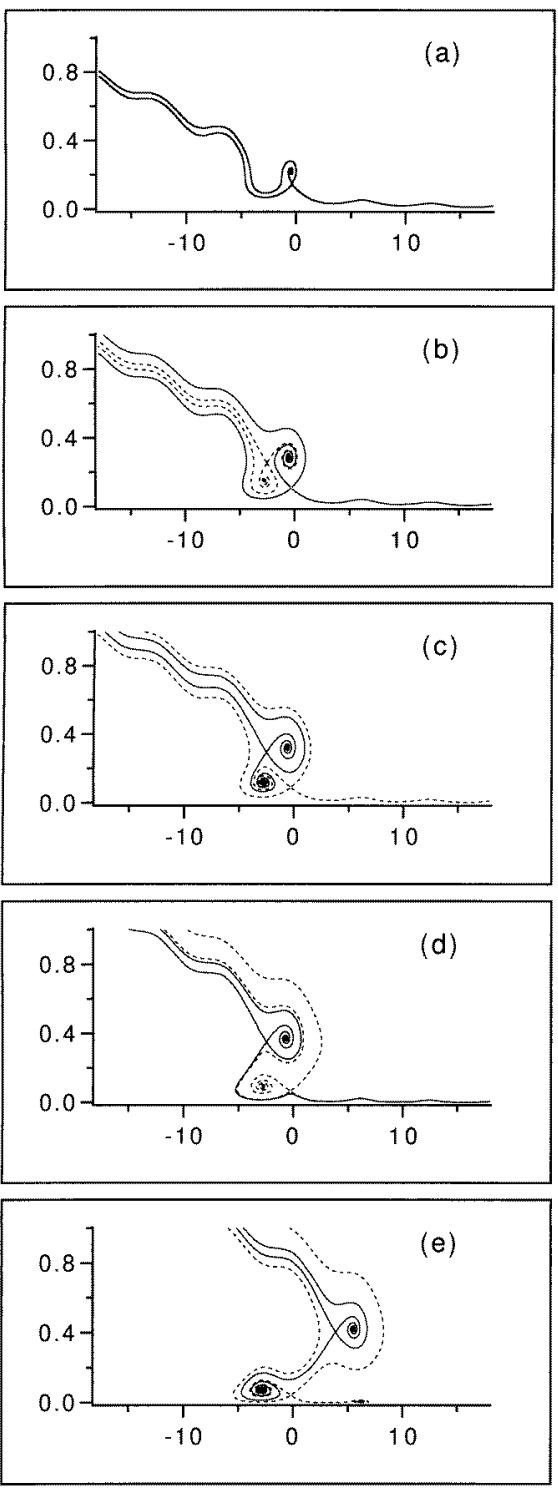

Figure 56. Calculated evolution with driving frequency $\omega_{f}$ of the basins of attraction (BAs) of nonlinear responses in a $2 \pi$ band of the phase space of the slow variables ( $I$ ordinate, $\tilde{\psi}$ abscissa) for the same system as in figure 55 but averaged over the high-frequency oscillations (equations (147) and (148)) for $h=0.0143$ and: (a) $\omega_{f}=0.8$; (b) 0.83 ; (c) 0.85 ; (d) 0.88 ; (e) 0.92 . The boundaries of the BAs of S2 (S3) and trajectories emerging from the saddles are drawn by full (dashed) curves. One can obtain the complete phase space by repeating the above picture with a period $2 \pi$ in $\tilde{\psi}$. (Luchinsky et al 1996a).

around the central cusp point (figure 54, inset). Because the separation between the cusp and the lower full curve tends towards zero as $\Gamma \rightarrow 0$, however, this possibility can usually be ignored. Beyond the close vicinity of the cusp, the frequency $\omega_{f}^{(\text {tr) }}$ of the ZDNR/NR transition can be shown to satisfy an asymptotic $(h \rightarrow 0)$ formula which is valid for the 
general case rather than for (152) only:

$$
\begin{aligned}
& \omega_{f}^{(\mathrm{tr})}=\omega_{\mathrm{m}}+\operatorname{sgn}\left(\omega_{\mathrm{m}}^{\prime \prime}\right)\left(\left|\omega_{\mathrm{m}}^{\prime \prime}\right| / 2\right)^{1 / 3}\left[\frac{3}{2} h x_{1 \mathrm{~m}}\left\{\left(1-\eta^{2}\right)^{1 / 2}-\eta\left(\frac{\pi}{2}-\arcsin (\eta)\right)\right\}\right]^{2 / 3} \\
& \eta \equiv \frac{\Gamma I_{\mathrm{m}}}{h x_{1 \mathrm{~m}}}<1 \quad\left[\frac{\Gamma}{\left|\omega_{\mathrm{m}}^{\prime \prime}\right| I_{\mathrm{m}}^{2}}\right]^{2 / 3} \ll\left[\frac{\omega_{f}^{(\mathrm{rr})}-\omega_{\mathrm{m}}}{\omega_{\mathrm{m}}^{\prime \prime} I_{\mathrm{m}}^{2}}\right]^{1 / 2} \ll 1 .
\end{aligned}
$$

It can be found more exactly by numerical solution of (147) (figure 54).

There is another non-trivial bifurcation that has no analogue in the dissipationless case: the alternation between the BA of one attractor either encompassing the other one, or simply moving around it (figures 55(c)-(e)), as the frequency is changed (for smaller $\Gamma$, a similar alternation takes place at the ZDNR stage too). These bifurcations are of the saddle connection type too and, together with the ZDNR/NR transition, they are characteristic of any oscillatory system whose variation of eigenfrequency with energy possesses an extremum. The ZDNR/NR and encompassing/moving around bifurcations are of particular interest because they may be expected to give rise to unusual fluctuational phenomena.

Of course, in the presence of noise, previously stable states become metastable. Escape from an attractor takes place with overwhelming probability via one of the saddle points of its BA (Dykman and Krivoglaz 1979, Freidlin and Wentzell 1984). Thus, the transition of a saddle point from the BA of one nonlinear response to the other at the ZDNR/NR bifurcation would be expected to result in a jump-wise change in the probabilities of fluctuational transitions between the nonlinear and linear responses (in figures 56(b) and (c), the trajectory outgoing to the right from the saddle point with a lower action goes to the attractor corresponding to linear response): for ZDNR, just before the bifurcation, there are no direct transitions between attractors $\mathrm{S} 1$ and $\mathrm{S} 2$, whereas, for NR, just after the bifurcation, there are no direct transitions between S1 and S3. A similar effect should occur at global bifurcations of the 'encompassing/moving around' type. Note that these various changes cannot be described in terms of frequency-response curves (Hayashi 1964) because nothing happens to the attractors themselves at a global bifurcation.

One may expect that the sequence of global bifurcations undergone by a ZD oscillator should manifest itself in some very unusual dependences of the fluctuational inter-attractor transition probabilities on parameters of the driving force. They have yet to be studied in detail, but it is interesting to consider briefly stationary fluctuations in the system. It can be shown that, for small enough $h$, there is a repopulation of the attractors associated with the ZDNR/NR transition: during the ZDNR stage, the population of attractor S3 is bigger than that of S2, whereas it is the other way round for NR. As discussed by Luchinsky et al (1995), this repopulation produces corresponding changes in the fluctuation spectra which are at their most pronounced for intermediate noise intensities and amplitudes of the driving force. The spectral density of fluctuations of the coordinate consists typically of a narrow transition peak at the frequency of the driving force which is due to fluctuational transitions between the metastable states, Stokes and anti-Stokes bands which are due to small fluctuations around S2 and S3, and a comparatively broad peak at $\omega_{0}$ caused by small fluctuations near S1 (cf Dykman and Krivoglaz 1979, 1984, Dykman et al 1989). The repopulation of S2 and S3 reverses the relative amplitudes of the Stokes and anti-Stokes bands and can also result in a rather unusual dependence of the intensity of the transition peak on the frequency of the driving force: quite unlike the case of the Duffing oscillator (Dykman et al 1994c), it can have a two-humped structure. 
Studies of ZDNR and associated phenomena are still in their infancy, and many puzzles remain to be resolved including, in particular, those associated with chaos (Neiman et al 1995) and with fluctuational effects (Luchinsky et al 1995). It seems likely that the former will be treated mainly via digital approaches, and the latter mainly via analogue experiments of the kind discussed in this review.

\section{Future directions}

The examples discussed above are intended to convey some idea of the versatility, convenience and power of analogue electronic modelling. Despite its simplicity, the technique can be applied to problems at the forefront of statistical mechanics (see, e.g., section 3) as well as to a wide range of applied research. It is of particular value on occasions when nonlinear dynamical systems are to be explored and their behaviour has to be qualitatively understood over a wide range of parameters, for instance in the development of the Josephson junction voltage standard (Kautz 1996), analyses of the action potential in the giant axon of a squid (Starzak 1984), studies of chaotic behaviour in nonlinear oscillators (see, e.g., references in section 1 and at the start of section 7), investigations of stochastic resonance (section 4) and the pilot experiments (section 4.6) preparing the way for studies of noise-enhanced optical heterodyning (Dykman et al 1995a). Given that the technique needs only a small addition-usually just a handful of standard cheap ICs - to the equipment already available in most laboratories, we suggest that it could with advantage be applied much more widely, in undergraduate teaching as well as in research.

What of the future? Well, in the long term, one can guess that the advantage in speed sometimes held by analogue over digital methods at the moment will probably disappear as processor speeds inexorably rise. Of course, the frequency responses and quality of analogue components (e.g. multipliers) are also likely to improve, permitting faster operation of the analogue models. Nonetheless, part of their present convenience lies in the possibility of creating them on simple open mounting boards with pressure contacts, thereby setting an effective upper frequency limit of perhaps $\sim 1 \mathrm{MHz}$ : at frequencies much higher than this stray capacitance and inductance, and phase shifts across the circuit, become important and very careful component layout on a printed circuit board is then essential. However, there are good reasons to suppose that—even without its present speed advantage- the peculiar merits of the analogue technique will ensure its continued use for the forseeable future in many areas of science and technology. Of the features discussed in section 1, the most relevant here is the close relationship to real experiments and the possibility to explore, in real time and visually, a broad range of parameter values.

In the medium term, one can be confident in predicting that research on stochastic systems of all kinds will continue to be extremely active in exploiting the advantages of analogue techniques. One of the fastest developing areas is the physics of large rare fluctuations (sections 3 and 6), which is still at a relatively early stage of its evolution, and where we forsee intense activity over the coming decade, with theory and experiment advancing together in a mutually supportive way. Up to now, analogue models have constituted the only experimental means of investigating the dynamics of large fluctuations: the nonlinear dynamics of large fluctuations would probably not have reached its current status as a subject area without the possibility of observing optimal fluctuational paths, together with the singularities in their pattern, and the shape of the tube of fluctuational paths. In addition to further intensive experimentation of a similar kind during the next few years, we anticipate that optimal paths will also be observed and studied in natural systems—using 
ideas and techniques, for example measurements of the prehistory probability distribution, already developed and tested on the analogue models.

Important stochastic problems ripe for immediate investigation using the techniques described above include, for example: the relationship between singularities of the pattern of optimal paths and theories of phase transitions, including the dynamics of soft modes near critical points; the recently introduced theory of the logarithmic susceptibility (Dykman et al 1997a, Smelyanskiy et al 1997b), which urgently requires experimental verification/testing, especially in the context of a variety of diverse applications, for example the selective control of diffusion by ac fields; and the characteristic behaviour expected near nascent cusp points of infinite codimension (Maier and Stein 1996a), including saddle-point avoidance and nonGaussian exit location distributions (section 3.3.3). Amongst applications of the basic ideas and results on fluctuating nonlinear systems are numerous biological problems, in addition to those representing applications of stochastic resonance (Bulsara et al 1995, Collins et al 1995, 1996, Wiesenfeld and Moss 1995), that are already yielding, or likely to be amenable, to analogue approaches. They exist on all length/complexity scales from the molecular level up to the complete organism. Examples include the old problem of the passage of currents through ion channels in a cell membrane (Eisenberg et al 1995, Eisenberg 1996a, b), Brownian ratchets (Astumian 1997), and the relationship between noise and chaos in the human cardiovascular system (Camm et al 1996).

Analogue modelling techniques of the kind described in this review seem certain to play an important role in the solution of these and of other problems, doubtless including many that are as yet unformulated, in the years to come.

\section{Acknowledgments}

We freely acknowledge our very considerable indebtedness to Riccardo Mannella, Norman Stein and Nigel Stocks, on the experimental side, and to the late Michael A Krivoglaz and to Vadim Smelyanskiy, on the theoretical side, as well as to many other collaborators, including Manuel Arrayás, Vladimir Chinarov, Xiaolin Chu, Richard Dow, Igor Fedchenia, Leo Fronzoni, Genadi-Petrovich Golubev, Paolo Grigolini, Hermann Haken, Peter Hänggi, Werner Horsthemke, Hu Gang, Paul Hunt, Akito Igarashi, Jan Iwaniszewski, Mark James, Igor Kaufman, Dilip Kondepudi, Colin Lambert, Stuart Lawson, Richard Lee, Katja Lindenberg, Luigi Lugiato, Robert Maier, Paolo Martano, Alan McKane, Mark Millonas, Eugenia Mori, Frank Moss, Shura Neiman, Takehiko Norita, Herschel Rabitz, the late Hans Risken, Stuart Robinson, John Ross, Jose-Maria Sancho, Hiroko Short, Slava Soskin, Daniel Stein, Alexander Velikovich and Boris Vugmeister. Without their collaboration, the research described above could not have been undertaken. The work has been supported in part by the Engineering and Physical Sciences Research Council (UK), the Royal Society of London, the National Science Foundation (USA), the North Atlantic Treaty Organisation, the European Community, the International Science Foundation, INTAS and the Russian Foundation for Fundamental Research.

\section{References}

Anishchenko V S, Neiman A B and Safanova M A 1993 J. Stat. Phys. 70 183-96

Anishchenko V S, Safanova M A and Chua L 1994 Int. J. Bifurcation Chaos 4 441-46

Arecchi F T and Lisi F 1982 Phys. Rev. Lett. 49 94-7

Arecchi F T, Politi A and Ulivi L 1982 Nuovo Cimento B 71 119-54

Arimondo E, Dangoisse D and Fronzoni L 1987 Europhys. Lett. 4 287-92

Arnol'd V I 1978 Mathematical Methods of Classical Mechanics (Berlin: Springer) 
Arnol'd V I 1992 Catastrophe Theory (Berlin: Springer)

Arrayás M, Casado J M, Gómez Ordóñez J, McClintock P V E, Morillo M and Stein N D 1998a Phys. Rev. Lett. $802273-6$

Arrayás M, Mannella R, McClintock P V E, McKane A J and Stein N 1997 Current reversals in a ratchet driven by quasimonochromatic noise Noise in Physical Systems and $1 / f$ Fluctuations ed C Claeys and E Simoen (Singapore: World Scientific) pp 381-4

1998 Current reversal in a correlation ratchet, to be published

Astumian R D 1997 Science 276 917-22

Astumian R D and Bier M 1994 Phys. Rev. Lett. 72 1766-9

Baras F, Nicolis G, Mansour M M and Turner J W 1983 J. Stat. Phys. 32 1-23

Barone R and Paterno G 1982 Physics and Applications of the Josephson Effect (New York: Wiley)

Barton D 1991 Physics World 4 56-60

Beasley M R, D’Humieres D, Huberman B A, Vos R F, Arecchi F T and Lisi F 1983 Phys. Rev. Lett. 50 1328-30

Ben-Jacob E, Bergman D J, Matkowsky B J and Schuss Z 1982 Phys. Rev. A 26 2805-16

Benzi R, Sutera S and Vulpiani A 1981 J. Phys. A: Math. Gen. 14 L453-L457

Berry M V 1976 Adv. Phys. 25 1-26

1977 J. Phys. A: Math. Gen. 10 2061-81

Bezrukov S M and Vodyanoy I 1995 Nature 378 362-4

-1997 Nature $385319-21$

Binder K 1973 Phys. Rev. B 8 3423-8

Boltzmann L 1904 On statistical mechanics (address given to the Scientific Congress in St Louis, 1904) reprinted in Theoretical Physics and Philosophical Problems ed B McGuinness (Dordrecht: Reidel) pp 159-72

Brand H R, Doering C R and Ecke R E 1989 J. Stat. Phys. 54 1111-19

Bray A J and McKane A J 1989 Phys. Rev. Lett. 62 493-6

Bray A J, McKane A J and Newman T J 1990 Phys. Rev. A 41 657-67

Bryant P and Wiesenfeld K 1986 Phys. Rev. A 33 2525-43

Bryant P, Wiesenfeld K and McNamara B 1987 Phys. Rev. A 36 752-5

Broggi G and Lugiato L A 1984a Phys. Rev. A 29 2949-52 1984b Phil. Trans. R. Soc., London A 313 425-8

Broggi G, Lugiato L A and Colombo A 1985 Phys. Rev. A 32 2803-2812

Bulsara A R, Chillemi S, Kiss L B, McClintock P V E, Mannella R, Marchesoni F, Nicolis C and Wiesenfeld K (eds) 1995 Fluctuations in physics and biology: stochastic resonance, signal processing and related phenomena Nuovo Cimento D 17 653-987

Bulsara A R and Gammaitoni L 1996 Physics Today March 39-45

Camm A J et al 1996 Circulation 93 1043-65

Caroli B, Caroli C and Roulet B 1980 Physica A 101 581-7

Carroll T L and Pecora L M 1993 Phys. Rev. Lett. 70 576-9

Casademunt J, Jiménez-Aquino J I and Sancho J M 1989a Physica A 156 628-50 1989b Phys. Rev. A 40 5905-14

Casademunt J, Jiménez-Aquino J I, Sancho J M, Lambert C J, Mannella R, Martano P, McClintock P V E and Stocks N G 1989c Phys. Rev. A 40 5915-21

Casademunt J, Mannella R, McClintock P V E, Moss F and Sancho J M 1987 Phys. Rev. A 35 5183-90

Casademunt J and Sancho J M 1989 Phys. Rev. A 39 4915-18

Chinarov V A, Dykman M I and Smelyanskiy V N 1993 Phys. Rev. E 47 2448-61

Cline D B (ed) 1996 Physical Origin of the Homochirality of Life (Washington, DC: American Institute of Physics)

Colet P, De Pasquale F and San Miguel M 1991 Phys. Rev. A 43 5296-307

Collins J J, Chow C C and Imhoff T T 1995 Nature 376 236-8

Collins J J, Imhoff T T and Grigg P 1996 J. Neurophys. 76 642-5

Cross M C and Hohenberg P C 1993 Rev. Mod. Phys. 65 851-1112

Day M V 1987 Stochastics 20 121-50

Debnath G, Zhou T and Moss F 1989 Phys. Rev. A 39 4323-6

Debye P 1929 Polar Molecules (New York: Dover)

Dehmelt H 1990 Rev. Mod. Phys. 62525

DeWeese M and Bialek W 1995 Nuovo Cimento D 17 733-42

D’Humieres D, Beasley M R, Huberman B A and Libchaber A 1982 Phys. Rev. A 26 3483-96

Doering C, Horsthemke W and Riordan J 1994 Phys. Rev. Lett. 72 2984-7

Douglass J K, Wilkens L, Pantazelou E and Moss F 1993 Nature 365 337-441

Dow R C M, Lambert C J, Mannella R and McClintock P V E 1987 Phys. Rev. Lett. 59 6-9

Drummond P D and Walls D F 1980 J. Phys. A: Math. Gen. 13 725-41 
Dykman M I 1990 Phys. Rev. A 42 2020-9

Dykman M I, Golubev G P, Kaufman I Kh, Luchinsky D G, McClintock P V E and Zhukov E A 1995a Appl. Phys. Lett. 67 308-10

-1995b Nuovo Cimento D 17 743-54

Dykman M I, Golubev G P, Luchinsky D G, McClintock P V E, Stein N D and Stocks N G 1994a Phys. Rev. E 49 1935-42

Dykman M I, Haken H, Gang Hu, Luchinsky D G, Mannella R, McClintock P V E, Ning C Z, Stein N D and Stocks N G 1993a Phys. Lett. 180A 332-6

Dykman M I, Horita T and Ross J 1995c J. Chem. Phys. 103 966-72

Dykman M I and Krivoglaz M A 1971 Phys. Status Solidi b 48 497-512

-1979 Sov. Phys.-JETP 50 30-7

1984 Soviet Physics Reviews vol 5, ed I M Khalatnikov (New York: Harwood) pp 265-441

Dykman M I, Krivoglaz M A and Soskin S M 1989 Noise in Nonlinear Dynamical Systems vol 2, ed F Moss and P V E McClintock (Cambridge: Cambridge University Press) pp 347-80

Dykman M I and Lindenberg K 1994 Contemporary Problems in Statistical Physics ed G H Weiss (Philadelphia, PA: SIAM) pp 41-101

Dykman M I, Luchinsky D G, Mannella R, McClintock P V E, Short H E, Stein N D and Stocks N G 1994b Phys. Lett. 193A 61-6

Dykman M I, Luchinsky D G, Mannella R, McClintock P V E, Soskin S M and Stein N D 1996a Phys. Rev. E 54 2366-77

Dykman M I, Luchinsky D G, Mannella R, McClintock P V E, Stein N D and Stocks N G 1993b Sov. Phys.-JETP Lett. 58 150-6

-1993c J. Stat. Phys. $70463-78$

-1993d J. Stat. Phys. 70 479-99

-1994c Phys. Rev. E 49 1198-215

-1994d Modern Nonlinear Optics (Advances in Chemical Physics Series vol LXXXV) part 3, ed M W Evans and S Kielich (New York: Wiley) pp 265-378

-1995d Nuovo Cimento D 17 661-83

-1995e Appl. Nonlinear Dynam. 3 56-69

1996b Fluctuations and Order: the New Synthesis ed M M Millonas (Berlin: Springer) pp 11-33

Dykman M I, Luchinsky D G, McClintock P V E and Smelyanskiy V N 1996c Phys. Rev. Lett. 77 5229-32

Dykman M I, Luchinsky D G, McClintock P V E, Stein N D and Stocks N G 1992a Phys. Rev. A 46 R1713-R1716

Dykman M I, Mannella R, McClintock P V E, Moss F and Soskin S M 1988 Phys. Rev. A 37 1303-13

Dykman M I, Mannella R, McClintock P V E, Soskin S M and Stocks N G 1990a Europhys. Lett. 13 691-6

-1990b Phys. Rev. A 42 7041-9

-1991a Phys. Rev. A 43 1701-8

Dykman M I, Mannella R, McClintock P V E, Stein N D, Luchinsky D G and Short H E 1995 f Nuovo Cimento D 17 755-64

Dykman M I, Mannella R, McClintock P V E, Stein N D and Stocks N G 1993e Phys. Rev. E 47 3996-4009

1993f Phys. Rev. E 47 1629-32

Dykman M I, Mannella R, McClintock P V E and Stocks N G 1990c Phys. Rev. Lett. 652606

-1990d JETP Lett. 52 141-4

-1990e Phys. Rev. Lett. 65 48-51

-1992b Phys. Rev. Lett. 68 2985-8

-1993g Phys. Rev. Lett. 70874

Dykman M I and McClintock P V E 1992 Physica D 58 10-30

-1998 Nature 391344

Dykman M I, McClintock P V E, Smelyanskiy V N, Stein N D and Stocks N G 1991b Phys. Rev. Lett. 67 933-6

1992c Phys. Rev. Lett. 68 2718-21

Dykman M I, Millonas M M and Smelyanskiy V N 1994e Phys. Lett. 195A 53-8

Dykman M I, Mori E, Ross J and Hunt P M 1994f J. Chem. Phys. 100 5735-50

Dykman M I, Rabitz H, Smelyanskiy V N and Vugmeister B E 1997a Phys. Rev. Lett. 79 1178-81

Dykman M I and Smelyanskiy V N 1998a Superlatt. Microstruct. 24

1998 b, in preparation

Dykman M I, Smelyanskiy V N, Lambert C J, Luchinsky D G and McClintock P V E 1997b Applied Nonlinear Dynamics and Stochastic Systems near the Millenium ed J B Kadtke and A Bulsara (Woodbury, NY: American Institute of Physics) pp 157-62

Dykman M I, Smelyanskiy V N, Luchinsky D G, Mannella R, McClintock P V E and Stein N D 1997c Large fluctuations in a periodically driven dynamical system Int. J. Bifurcation Chaos at press 
Dykman M I, Velikovich A L, Golubev G P, Luchinsky D G and Tsuprikov S V 1991c Sov. Phys.-JETP Lett. 53 193-7

Ebeling W and Schimansky-Geier L 1989 Noise in Nonlinear Dynamical Systems vol 1, ed F Moss and P V E McClintock (Cambridge: Cambridge University Press) pp 279-306

Einchcomb S J B and McKane A J 1994 Phys. Rev. E 49 259-66

-1995 Phys. Rev. E 51 2974-81

Einstein A 1905 Ann. Phys., Lpz. 17 549-60

1910 Ann. Phys., Lpz. 33 1275-98

Eisenberg R S 1996a Recent Developments in Theoretical Studies of Proteins ed R Elber (Philadelphia: World Scientific) pp 269-357

-1996b J. Membrane Biol. 150 1-25

Eisenberg R S, Kłosek M M and Schuss Z 1995 J. Chem. Phys. 102 1767-80

Faetti S, Festa C, Fronzoni L and Grigolini P 1985 Memory Function Approaches to Stochastic Problems in Condensed Matter ed M W Evans, P Grigolini and G Pastori-Paravicini (New York: Wiley) pp 445-75

Faetti S, Festa C, Fronzoni L, Grigolini P and Martano P 1984 Phys. Rev. A 30 3252-63

Fauve S and Heslot F 1983 Phys. Lett. 97A 5-7

Fedchenia I I, Mannella R, McClintock P V E, Stein N D and Stocks N G 1992 Phys. Rev. A 46 1769-74

Feynman R P and Hibbs A R 1965 Quantum Mechanics and Path Integrals (New York: McGraw-Hill)

Fioretti A, Guidoni L, Mannella R and Arimondo E 1993 J. Stat. Phys. 70 403-12

Freeman W 1988 Electronic Product Design December 18-20

Freidlin M I and Wentzell A D 1984 Random Perturbations in Dynamical Systems (New York: Springer)

French A S, Holden A V and Stein R B 1972 Kybernetik 11 15-23

Flytzanis C and Tang C L 1980 Phys. Rev. Lett. 45 441-5

Fox R F 1989 Phys. Rev. A 39 4148-53

Frankowicz M and Nicolis G 1983 J. Stat. Phys. 33 595-609

Frankowicz M, Mansour M M and Nicolis G 1984 Physica A 125 237-46

Fronzoni L 1989 Noise in Nonlinear Dynamical Systems vol 3, ed F Moss and P V E McClintock (Cambridge: Cambridge University Press) pp 222-42

Fronzoni L, Grigolini P, Hanggi P, Moss F, Mannella R and McClintock P V E 1986 Phys. Rev. A 33 3320-7

Fronzoni L, Grigolini P, Mannella R and Zambon B 1985 J. Stat. Phys. 41 533-79

Fronzoni L, Mannella R, McClintock P V E and Moss F 1987a Phys. Rev. A 36 834-41

Fronzoni L, Moss F and McClintock P V E 1987b Phys. Rev. A 36 1492-4

Gabrielse G, Dehmelt H and Kells W 1985 Phys. Rev. Lett. 54 537-39

Gammaitoni L, Hänggi, Jung P and Marchesoni F 1998 Rev. Mod. Phys. 70 223-88

Gammaitoni L, Marchesoni F, Martinelli M, Pardi L and Santucci S 1991a Phys. Lett. 158A 449-52

Gammaitoni L, Marchesoni F, Menichella-Saetta E and Santucci S 1989 Phys. Rev. Lett. 62 349-52

-1995 Nuovo Cimento D 17 785-93

Gammaitoni L, Martinelli M, Pardi L and Santucci S 1991b Phys. Rev. Lett. 67 1799-802

Gibbs H M 1985 Optical Bistability: Controlling Light with Light (New York: Academic Press)

Gingl Z, Kiss L B and Moss F 1995 Europhys. Lett. 29 191-6

Goldstone J A and Garmire E 1984 Phys. Rev. Lett. 53 910-13

Golomb S 1967 Shift Register Sequences (San Francisco: Holden-Day)

Gomes M G M and King G P 1992 Phys. Rev. A 46 3100-110

Graham R 1973 Quantum Statistics in Optics and Solid-State Physics (Springer Tracts in Modern Physics 66) ed G Höbler (Berlin: Springer) pp 1-97

Graham R 1989 Noise in Nonlinear Dynamical Systems vol 1, ed F Moss and P V E McClintock (Cambridge: Cambridge University Press) pp 225-78

Graham R and Tél T 1984a Phys. Rev. Lett. 52 9-12

-1984b J. Stat. Phys. 35 729-48

-1985 Phys. Rev. A 31 1109-122

-1986 Phys. Rev. A 33 1322-37

-1990 Phys. Rev. A 42 4661-77

Grigolini P 1989 Noise in Nonlinear Dynamical Systems vol 1, ed F Moss and P V E McClintock (Cambridge: Cambridge University Press) pp 161-90

Grigolini P, Lugiato L A, Mannella R, McClintock P V E, Merri M and Pernigo M 1988 Phys. Rev. A 38 1966-78

Grigorenko A N, Nikitin P I and Roshchepkin G V 1997 JETP Lett. 65 828-32

Grohs J, Apanasevich S, Jung P, Issler H, Burak D and Klingshirn C 1994 Phys. Rev. A 49 2199-202

Guckenheimer J and Holmes P 1983 Nonlinear Oscillations, Dynamical Systems and Bifurcations of Vector Fields (New York: Springer)

Gwaki L N, Lambert C J, Mannella R and McClintock P V E 1993 Phys. Rev. B 47 14 200-5 
Haken H 1975 Rev. Mod. Phys. 47 67-119

1977 Synergetics, An Introduction (Berlin: Springer)

Hänggi P 1989 Noise in Nonlinear Dynamical Systems vol 1, ed F Moss and P V E McClintock (Cambridge: Cambridge University Press) pp 307-28

Hänggi P, Mroczowski T J, Moss F E and McClintock P V E 1985 Phys. Rev. A 32 695-8

Hayashi C 1964 Nonlinear Oscillations in Physical Systems (New York: McGraw-Hill)

Heagy J F, Carroll T L and Pecora L M 1994 Phys. Rev. Lett. 50 1874-85

Hegstrom R A and Kondepudi D K 1990 Scientific American January 98-105

Hibbs A D, Singsaas A L, Jacobs E W, Bulsara A R, Bekkedahl J J and Moss F 1995 J. Appl. Phys. 77 2582-90

Hohenberg P C and Halperin B I 1977 Rev. Mod. Phys. 49 435-79

Holmes P 1979 Phil. Trans. R. Soc. A 292 419-48

Hondou T and Sawada Y 1995 Phys. Rev. Lett. 75 3269-72

Howard J E and Hohs S M 1984 Phys. Rev. A 29 418-21

Hunt P M, Hunt K L C and Ross J 1990 J. Chem. Phys. 92 2572-81

Iannelli J M, Yariv A, Chen T R and Zhuang Y J 1994 Appl. Phys. Lett. 65 1883-5

Igarashi A and Munakata T 1988 J. Phys. Soc. Japan 57 2439-47

Igarashi A, McClintock P V E and Stocks N G 1992 J. Stat. Phys. 66 1059-70

Ivanov M A, Kvashnina L B and Krivoglaz M A 1966 Sov. Phys. Solid State 7 1652-9

Iwaniszewski J 1992a Phys. Rev. A 45 8436-40

-1992b Phys. Lett. 171A 278-84

1995 Nuovo Cimento D 17 819-25

Iwaniszewski J, McClintock P V E and Stein N D 1994 Phys. Rev. E $503538-45$

Jackson P J, Lambert C J, Mannella R, Martano P, McClintock P V E and Stocks N G 1989 Phys. Rev. A 40 $2875-8$

Jauslin H R 1987a J. Stat. Phys. 42 573-85

1987b Physica A 144 179-91

Jeffries C and P'erez J 1982 Phys. Rev. A 26 2117-22

Jeffries C and Wiesenfeld K 1985 Phys. Rev. A 31 1077-84

Jiménez-Aquino J I, Casademunt J and Sancho J M 1988 Phys. Lett. 133A 364-8

Jost B M and Saleh B E A 1996 Opt. Lett. 21 287-9

Jung P 1993 Phys. Rep. 234 175-295

Jung P and Hänggi P 1991 Phys. Rev. A 44 8032-42

Kac M 1959 Probability and Related Topics in Physical Science (New York: Wiley-Interscience)

Kai S, Andoh M and Yamaguchi S 1992 Phys. Rev. A 46 R7375-R7378

Kaufman I Kh, Luchinsky D G, McClintock P V E, Soskin S M and Stein N D 1997 Phys. Rev. E 57 78-87

-1998 Phys. Lett. 220A 219-23

Kautz R L 1988 Phys. Rev. A 38 2066-80

1996 Rep. Prog. Phys. 59 935-92

Khovanov I A and Anishchenko V S 1997 Applied Nonlinear Dynamics and Stochastic Systems Near the Millenium ed J B Kadtke and A Bulsara (Woodbury, NY: American Institute of Physics) pp 267-72

Kim J-H and Lee H-W 1995 Phys. Rev. E 52 473-80

King G P and Gaito S T 1992 Phys. Rev. A 46 3092-9

Kłosek-Dygas M M, Matkowsky B J and Schuss Z 1988a SIAM J. Appl. Math. 48 425-41

- 1988b Phys. Rev. A 38 2605-13

-1989 J. Stat. Phys. 54 1309-20

Kondepudi D K, Moss F and McClintock P V E 1986a Physica D 21 296-306

-1986b Phys. Lett. A 114 68-74

Kondepudi D K and Nelson G W 1983 Phys. Rev. Lett. 50 1023-6

-1985 Nature 314 438-41

Kramers H 1940 Physica (Utrecht) 7 284-304

Kubo R, Toda M and Hashitsume N 1978 Statistical Physics II (Berlin: Springer)

Landau L D and Lifshitz E M 1976 Mechanics (London: Pergamon)

1980 Statistical Physics 3rd edn, part 1 (New York: Pergamon)

Landauer R 1962 J. Appl. Phys. 33 2209-16

-1975 J. Stat. Phys. 13 1-16

-1978 Phys. Lett. 68A 15-16

-1988 J. Stat. Phys. $\mathbf{5 3} 233-48$

Lange W 1988 Instabilities and Chaos in Quantum Optics II (NATO ASI Series B 177) ed N B Abraham, F T Arecchi and L A Lugiato (New York: Plenum) pp 265-80 
Lange W, Mitschke F, Deserno R and Mlynek J 1985 Phys. Rev. A 32 1271-4

Lebowitz J L 1993 Physics Today 46 (9) 32-8

Leibler S 1994 Nature 370 412-13

Lemarchand A, Ben Aim R I and Nicolis G 1989 Chem. Phys. Lett. 162 92-8

Leonard D S and Reichl L E 1994 Phys. Rev. E 49 1734-9

Lindenberg K, West B J and Masoliver J 1989a Noise in Nonlinear Dynamical Systems vol 1, ed F Moss and P V E McClintock (Cambridge: Cambridge University Press) pp 110-60

Lindenberg K, West B J and Tsironis G 1989b Rev. Solid State Sci. 3 143-84

Lindner J F, Meadows B K, Ditto W L, Inchiosa M E and Bulsara A R 1995 Phys. Rev. Lett. 75 3-6

Linsay P 1981 Phys. Rev. Lett. 47 1349-52

Loerincz K, Gingl Z and Kiss L B 1996 Phys. Lett. 224A 63-7

Lofstedt R and Coppersmith S N 1994 Phys. Rev. Lett. 72 1947-50

Longtin A 1995 Nuovo Cimento D 17 835-46

Luchinsky D G 1997 J. Phys. A: Math. Gen. 30 L577-L583

Luchinsky D G, Maier R S, Mannella R, McClintock P V E and Stein D L 1997 Phys. Rev. Lett. 79 3109-12

- 1998, to be published

Luchinsky D G and McClintock P V E 1997 Nature 389 463-6

Luchinsky D G, McClintock P V E, Soskin S M and Mannella R 1995 Noise in Physical Systems and 1/f Fluctuations ed V Bareikis and R Katilius (Singapore: World Scientific) pp 705-8

-1996a Phys. Rev. Lett. 76 4453-7

Luchinsky D G, McClintock P V E, Soskin S M, Stein N D and Neiman A B 1996b Phys. Rev. E 53 4240-1

Luciani J F and Verga A D 1987 Europhys. Lett. 4 255-61

-1988 J. Stat. Phys. 50 567-97

Luckock H C and McKane A J 1990 Phys. Rev. A 42 1982-96

Lugiato L A, Broggi G and Colombo A 1986 Frontiers in Quantum Optics ed E R Pike and S Sarkar (Bristol: Hilger) pp 231-60

Magnasco M O 1993 Phys. Rev. Lett. 71 1477-81

Maier R S and Stein D L 1993a Phys. Rev. Lett. 71 1783-6

_ 1993b Phys. Rev. E 48 931-8

-1996a J. Stat. Phys. 83 291-357

-1996b Phys. Rev. Lett. 77 4860-3

—1997 SIAM J. Appl. Math. 57 752-90

Mannella R 1997 Numerical integration of stochastic differential equations Proc. Euroconf. on Supercomputation in Nonlinear and Disordered Systems ed L V Vázquez, F Tirando and C Martín (Singapore: World Scientific) pp 100-30

Mannella R, Faetti S, Grigolini P and McClintock P V E 1988 J. Phys. A: Math. Gen. 21 1239-52

Mannella R, Faetti S, Grigolini P, McClintock P V E and Moss F E 1986 J. Phys. A: Math. Gen. 19 L699-L704

Mannella R, Lambert C J, Stocks N G and McClintock P V E 1990 Phys. Rev. A 41 3016-20

Mannella R and McClintock P V E 1990 Contemp. Phys. 31 179-94

Mannella R, McClintock P V E and Moss F 1987a Phys. Lett. 120A 11-14

-1987b Europhys. Lett. 4 511-15

Mannella R, Moss F and McClintock P V E 1987c Phys. Rev. A 35 2560-6

Mantegna R N and Spagnolo B 1994 Phys. Rev. E 49 R1792-R1795

Marchesoni F 1996 Phys. Rev. Lett. 77 2364-7

Matkowsky B J and Schuss Z 1983 Phys. Lett. 95A 213-15

McClintock P V E and Moss F 1985 Phys. Lett. 107A 367-70

1989 Noise in Nonlinear Dynamical Systems vol 3, ed F Moss and P V E McClintock (Cambridge: Cambridge University Press) pp 243-74

McClintock P V E, Soskin S M, Stein N D and Stocks N G 1993 Phys. Rev. E 48 147-56

McKane A J 1989 Phys. Rev. A 40 4050-3

McKane A J, Luckock H C and Bray A J 1990 Phys. Rev. A 41 644-56

McNamara B and Wiesenfeld K 1989 Phys. Rev. A 39 4854-69

McNamara B, Wiesenfeld K and Roy R 1988 Phys. Rev. Lett. 60 2626-9

Mason S 1985 Nature 314 400-1

Millonas M M 1995 Phys. Rev. Lett. 74 10-13

Millonas M M (ed) 1996 Fluctuations and Order: the New Synthesis (Berlin: Springer)

Millonas M M and Dykman M I 1994 Phys. Lett. 183A 65-9

Mitschke F, Desorno R, Mlynek J and Lange W 1985 IEEE J. Quantum Electron. 21 1435-40

Morillo M, Casado J M and Gómez-Ordóñez J 1997 Phys. Rev. E 55 1521-4 
Morillo M and Gómez-Ordóñez J 1993 Phys. Rev. Lett. 71 9-11

Morillo M, Gómez-Ordónez J and Casado J M 1995 Phys. Rev. E 52 316-20

Morton J B and Corrsin S 1969 J. Math. Phys. 10 361-8

Moss F 1994 Contemporary Problems in Statistical Physics ed G H Weiss (Philadelphia: SIAM) pp 205-53

Moss F, Bulsara A R and Shlesinger M F (eds) 1993 J. Stat. Phys. 70 1-512

Moss F, Hanggi P, Mannella R and McClintock P V E 1986 Phys. Rev. A 33 4459-61

Moss F, Kondepudi D K and McClintock P V E 1985a Phys. Lett. 112A 293-6

Moss F and McClintock P V E 1985 Z. Phys. B 61 381-6

Moss F, McClintock P V E and Horsthemke W 1985b Phys. Rev. Lett. 54604

Moss F and Wiesenfeld K 1995 Scientific American 273 (2) 66-9

Moss F and Xing Pei 1995 Nature 376 211-2

Nalik J, Lange W and Mitschke F 1989 Appl. Phys. B 49 191-9

Neiman A B and Schimansky-Geier L 1994 Phys. Rev. Lett. 72 2988-91

Neiman A B, Soskin S M and McClintock P V E 1995 Noise in Physical Systems and 1/f Fluctuations ed V Bareikis and R Katilius (Singapore: World Scientific) pp 701-4

Nicolis C 1982 Tellus 34 1-9

Nicolis C and Nicolis G 1991 Phys. Rev. A 43 5720-3

Nicolis G and Prigogine I 1977 Self-Organisation in Nonequilibrium Systems (New York: Wiley)

Onsager L 1931 Phys. Rev. 37 405-26

Onsager L and Machlup S 1953 Phys. Rev. 91 1505-12

de Pasquale F and Mecozzi A 1985 Phys. Rev. A 31 2454-9

Peyton A J and Walsh V 1993 Analog Electronics with Op Amps (Cambridge: Cambridge University Press)

Presilla C, Marchesoni F and Gammaitoni L 1989 Phys. Rev. A 40 2105-13

Prost J, Chauwin J-F, Peliti L and Ajdari A 1994 Phys. Rev. Lett. 72 2652-5

Rácz Z 1976 Phys. Rev. B 13 263-70

Rand C 1994 Physics World 7 30-3

Rice O 1944 Bell Syst. Tech. J. 23 282-332

Risken H 1993 The Fokker-Planck Equation 2nd edn (Berlin: Springer)

Robinson F N H 1990 Int. J. Electron. 68 803-19

Robinson S D, Moss F E and McClintock P V E 1985 J. Phys. A: Math. Gen. 18 L89-L94

Ross J, Hunt K L C and Hunt P M 1992 J. Chem. Phys. 96 618-29

Rytov S M 1956a Sov. Phys.-JETP 2 217-24

-1956b Sov. Phys.-JETP 2 225-35

Sancho J M, Mannella R, McClintock P V E and Moss F 1985 Phys. Rev. A 32 3639-46

Sancho J M and San Miguel M 1989 Noise in Nonlinear Dynamical Systems vol 1, ed F Moss and P V E McClintock (Cambridge: Cambridge University Press) pp 72-109

Sancho J M, San Miguel M, Yamazaki H and Kawakubo T 1982 Physica A 116 560-72

Schimansky-Geier L and Zülicke Ch 1990 Z. Phys. B 79 451-60

Schulman L S 1991 Physica A 177 373-80

1996 Techniques and Applications of Path Integration 2nd edn (New York: Wiley)

Shen Y R 1984 The Principles of Nonlinear Optics (New York: Wiley)

Simon A and Libchaber A 1992 Phys. Rev. Lett. 68 3375-8

Slater G W, Guo H-L and Nixon G I 1997 Phys. Rev. Lett. 78 1170-3

Smelyanskiy V N and Dykman M I 1997 Phys. Rev. E 55 2516-21

Smelyanskiy V N, Dykman M I and Maier R S 1997a Phys. Rev. E 55 2369-91

Smelyanskiy V N, Dykman M I, Rabitz H and Vugmeister B E 1997b Phys. Rev. Lett. 79 3113-16

Smith H T J and Blackburn J A 1994 Phys. Rev. E 50 539-45

von Smoluchowski M 1906 Ann. Phys., Lpz. 21 756-80

Smythe J, Moss F E and McClintock P V E 1983a Phys. Rev. Lett. 51 1062-4

Smythe J, Moss F E, McClintock P V E and Clarkson D 1983b Phys. Lett. 97A 95-8

Soskin S M 1989 Physica A 155 401-29

-1992 Physica A 180 386-406

-1994 Phys. Rev. E 50 R44-R46

Soskin S M and Luchinsky D G 1995 Nuovo Cimento D 17 915-24

Soskin S M, Luchinsky D G, Mannella R, Neiman A B and McClintock P V E 1997 Int. J. Bifurcation Chaos 7 923-36

Spano P, Mecozzi A and Sapia A 1990 Phys. Rev. Lett. 64 3003-6

Spano M L, Wun-Fogle M and Ditto W L 1992 Phys. Rev. A 46 5253-6

Starzak M E 1984 The Physical Chemistry of Membranes (New York: Academic) 
Stocks N G 1995 Nuovo Cimento 17 925-40

Stocks N G, Lambert C J, Mannella R and McClintock P V E 1993a Phys. Rev. B 47 8580-7

Stocks N G, Lambert C J and McClintock P V E 1989a J. Stat. Phys. 54 1397-410

Stocks N G, Mannella R and McClintock P V E 1989b J. Stat. Phys. 54 1383-96

-1989c Phys. Rev. A 40 5361-9

-1990 Phys. Rev. A 42 3356-62

Stocks N G, McClintock P V E and Soskin S M 1993b Europhys. Lett. 21 395-400

Stocks N G, Stein N D and McClintock P V E 1993c J. Phys. A: Math. Gen. 26 L385-L390

Stocks N G, Stein N D, Short H E, McClintock P V E, Mannella R, Luchinsky D G and Dykman M I 1996 Fluctuations and Order: the New Synthesis ed M M Millonas (Berlin: Springer) pp 53-68

Stocks N G, Stein N D, Soskin S M and McClintock P V E 1992 J. Phys. A: Math. Gen. 25 L1119-L1125

Stratonovich R L 1963 Topics in the Theory of Random Noise vol 1 (New York: Gordon and Breach)

1967 Topics in the Theory of Random Noise vol 2 (New York: Gordon and Breach)

Suzuki M 1976 Progr. Theor. Phys. 56 77-94

Tan J and Gabrielse G 1993 Phys. Rev. A 483105

Testa J, Perez J and Jeffries C 1982 Phys. Rev. Lett. 48 714-17

Tomlinson G H and Galvin P 1975 Electron. Lett. 11 77-8

Torrent M C and San Miguel M 1988 Phys. Rev. A 38 245-51

Torrent M C, Sagués F and San Miguel M 1989 Phys. Rev. A 40 6662-72

Tsironis G and Grigolini P 1988 Phys. Rev. Lett. 61 7-10

Valle A, Pesquera L and Rodriguez M A 1990 Opt. Commun. 79 156-64

Van den Broeck C and Mandel P 1987 Phys. Lett. 122A 36-8

Van der Pol B and Van der Mark J 1928 Phil. Mag. 6 763-75

Van Kampen N G 1990 Stochastic Processes in Physics and Chemistry (Amsterdam: Elsevier)

Vaudelle F, Gazengel J, Rivoire G, Godivier X and Chapeau-Blondeau F 1998 J. Opt. Soc. Am. B to be published Vemuri G and Roy R 1989 Phys. Rev. A 39 4668-74

Ventcel' A D and Freidlin M I 1970 Russian Math. Surveys 25 1-55

Vilar J M G and Rubi J M 1996 Phys. Rev. Lett. 77 2863-6

Vogel K, Risken H, Schleich W, James M, Moss F, Mannella R and McClintock P V E 1987a J. Appl. Phys. 62 721-3

Vogel K, Risken H, Schleich W, James M, Moss F and McClintock P V E 1987b Phys. Rev. A 35 463-5

Vohra S T and Bucholtz F 1993 J. Stat. Phys. 70 413-21

Vohra S T, Fabiny L and Wiesenfeld K 1994 Phys. Rev. Lett. 72 1333-6

Vugmeister B E and Rabitz H 1997 Phys. Rev. E 55 2522-4

Vugmeister B E, Botina J and Rabitz H 1997 Phys. Rev. E 55 5338-42

Wiesenfeld K 1993 Physics World February 23-4

Wiesenfeld K and Moss F 1995 Nature 373 33-6

Wiesenfeld K, Pierson D, Pantazelou E, Dames C and Moss F 1994 Phys. Rev. Lett. 72 2125-9

Wio H S and Zanette D H 1993 Phys. Rev. E 47 384-7

Wio H S, Colet P, San Miguel M, Pesquera L and Rodríguez M A 1989 Phys. Rev. A 40 7312-24

Yeh W H and Kao Y H 1982 Phys. Rev. Lett. 49 1888-91

Zeghlache H, Mandel P and Van Den Broeck C 1989 Phys. Rev. A 40 286-94

Zhou T, Moss F and Jung P 1990 Phys. Rev. A 42 3161-9

Zwanzig R 1973 J. Stat. Phys. 9 215-20 